Master thesis - Electrical Engineering

\title{
A GO/FO Tool for Analyzing Quasi-optical Systems in Reception
}

Huasheng Zhang

4608364 
A GO/FO Tool for Analyzing Quasi-optical Systems in Reception (October 20, 2018)

Copyright $\odot 2018$ Huasheng Zhang

All rights reserved.

T̛UDelft

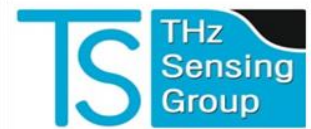




\section{Preface}

In this work, a MATLAB based graphical user interface (GUI) tool is built to analyze Quasioptical (QO) systems in reception with the Fourier Optics (FO) and Geometrical Optics (GO) methods. Five canonical QO components are discussed, namely parabolic reflectors, elliptical lenses, hemispherical lenses, hyperbolic lenses, and elliptical mirrors. The ray tracing technique is implemented to describe reception scenarios and visualize the phase aberrations in the QO systems. The FO method represents the fields focalized by a QO component on its focal plane as a Plane Wave Spectrum (PWS). This spectrum is calculated by using the GO method and can be used in spectral techniques like equivalent Floquet circuit. Moreover, the tool is able to use this spectrum to estimate the power delivered to an antenna placed at the focal plane. By obtaining this delivered power, the performance of the antenna-coupled QO system is analyzed, including pattern, directivity, common efficiency terms and gain. In addition, the performance calculated in reception is validated by CST and GRASP full-wave simulation software. Therefore, this GUI tool represents a GO/FO based tool that can be used to analyze and design antenna-coupled QO systems in reception.

This thesis is submitted in partial fulfillment of the requirements for the degree of Master of Science in Electrical Engineering at Delft University of Technology.

张华盛

Huasheng Zhang

Delft, October 2018 



\section{Acknowledgements}

First of all, I would like to thank prof. dr. Andrea Neto for giving me the opportunity to enter the Tera-Hertz Sensing Group and work on my thesis. At the beginning of my master study, I was attracted by your passion and expertise during lectures. And in the following study, I really learnt a lot from you.

I would like to thank prof. dr. Nuria Llombart who patiently guided me during my thesis project. You are the best supervisor I have ever seen. When I had questions, you were always patient with me and helped me out with your expertise in this field. I am really looking forward to working with you in the upcoming years.

Moreover, I want to express my most sincere gratitude to Shahab Dabironezare, my daily supervisor, who spent an immense amount of time on instructing me on various topics, from teaching me basic programming to modifying my thesis. I really appreciate your efforts on supervising me during this year.

I would also like to thank dr. Giorgio Carluccio for all his support. Your feedbacks on my problems and knowledge in electromagnetic theory really helped me a lot. I really learnt lots of things from you.

I would also like to thank my colleagues in the Tera-Hertz Sensing Group, for making such a nice working environment. I really enjoyed the lunch, coffee, and party time with you. Also, when I had questions, you always tried to help me no matter how busy you were.

Furthermore, I want to thank my dear friends who made my life in the Netherlands a lot happier. Especially I want to thank Ziqiao Huang, who always supported me and helped me get through my hard time.

Finally, I would like to express my most sincere gratitude to my parents and dedicate this thesis to them. You supported my study here and you were always there to give me advice and comforts. I feel really grateful, love you! 



\section{A GO/FO Tool for Analyzing Quasi-optical Systems in Reception}

This thesis is submitted in partial fulfillment of the requirements for the degree of

MASTER OF SCIENCE

in

ELECTRICAL ENGINEERING

by

Huasheng Zhang

The work presented in this thesis was performed at:

Tera-Hertz Sensing Group

Department of Microelectronics

Faculty of Electrical Engineering, Mathematics and Computer Science

Delft University of Technology 



\section{DELFT UNIVERSITY OF TECHNOLOGY DEPARTMENT OF ELECTRICAL ENGINEERING}

The undersigned hereby certify that they have read and recommended to the Faculty of Electrical Engineering, Mathematics and Computer Science for acceptance a thesis entitled "A GO/FO Tool for Analyzing Quasi-optical Systems in Reception" by Huasheng Zhang in partial fulfillment of the requirements for the degree of Master of Science.

Dated: Oct. 20, 2018

Chairman:

prof. dr. Andrea Neto

Supervisor:

prof. dr. Nuria Llombart Juan

Committee Member

dr. Rob Remis 


\section{Contents}

Preface

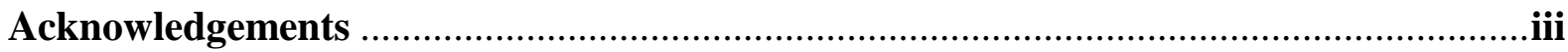

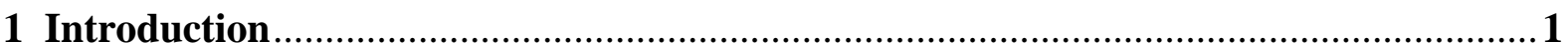

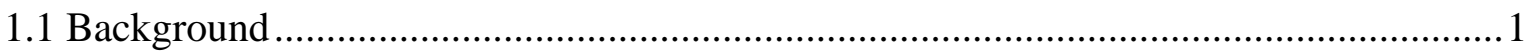

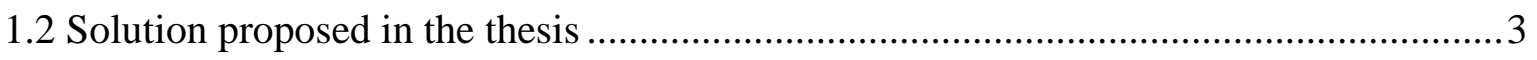

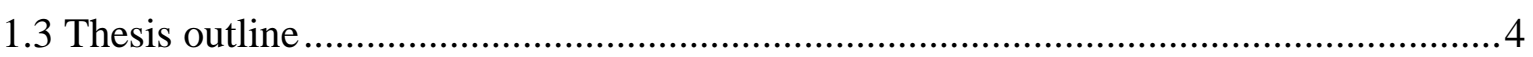

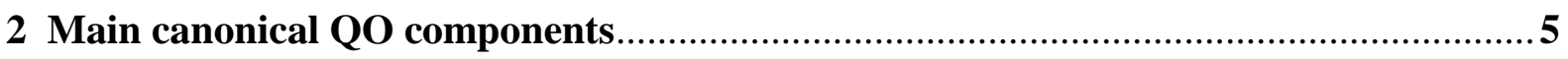

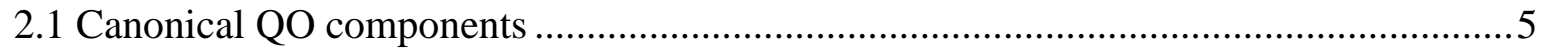

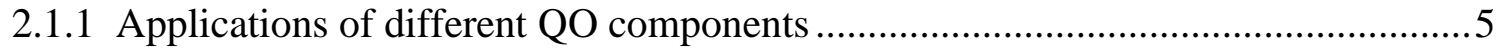

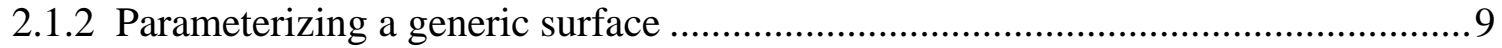

2.1.3 Parameterizing a specific QO component....................................................... 11

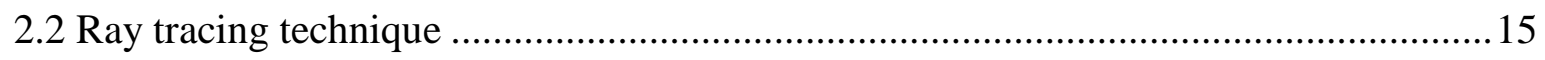

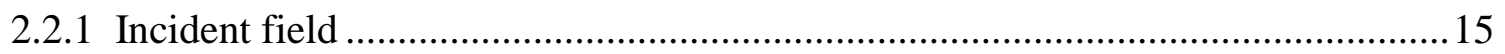

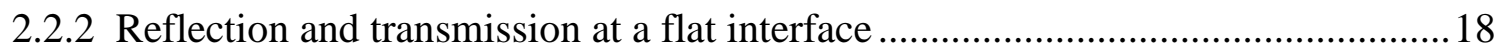

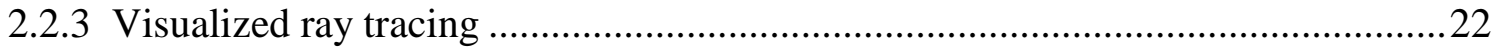

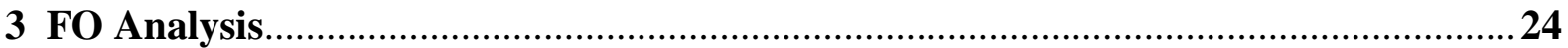

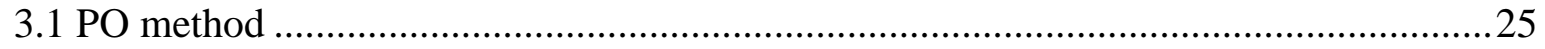

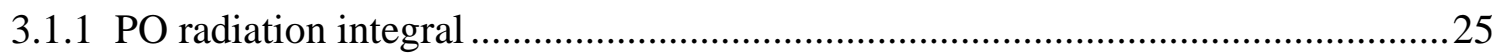

3.1.2 Validation of the implemented PO analysis..................................................26

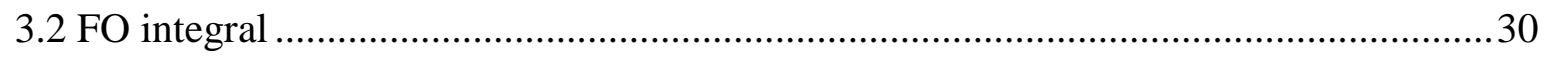

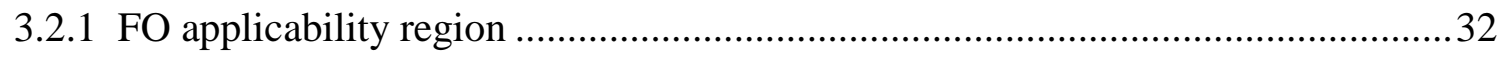

3.2.2 Parameterizing FO spheres for QO components.................................................. 32

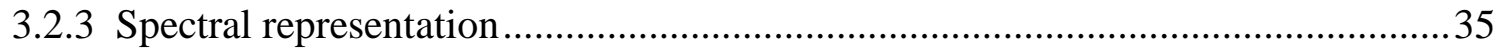

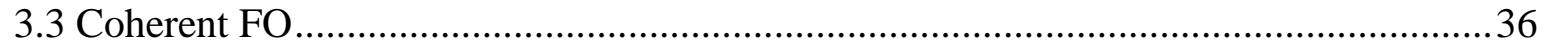

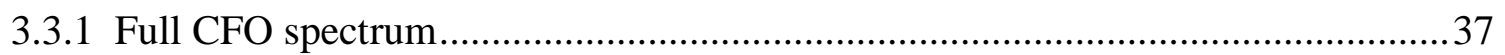

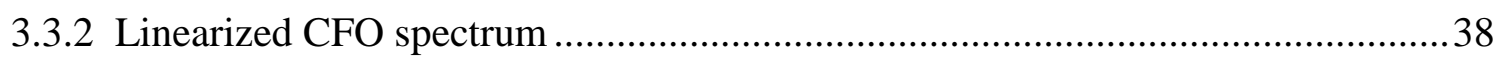

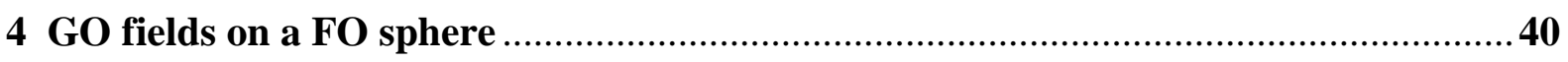

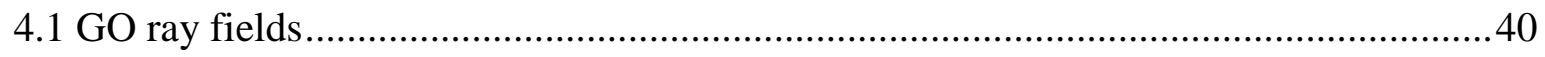

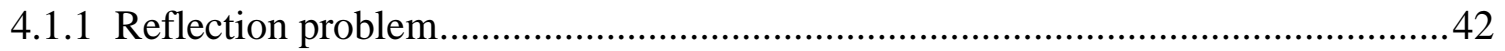

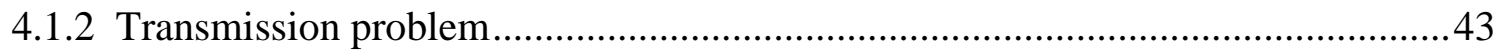

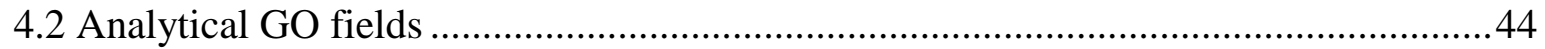

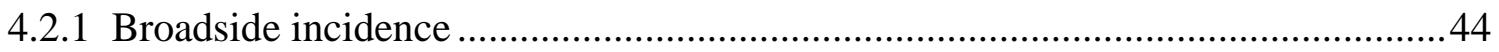


4.2.2 Slightly off-broadside incidence .57

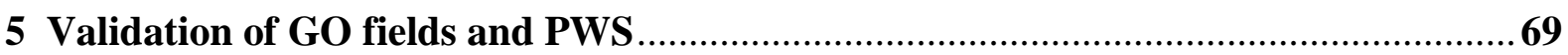

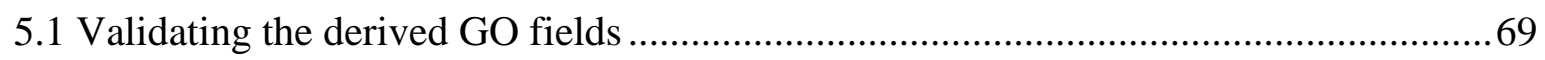

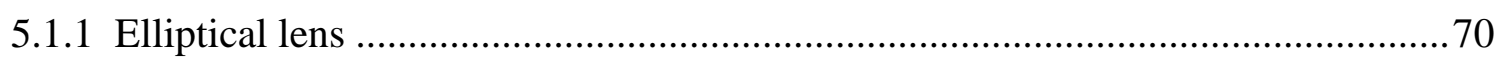

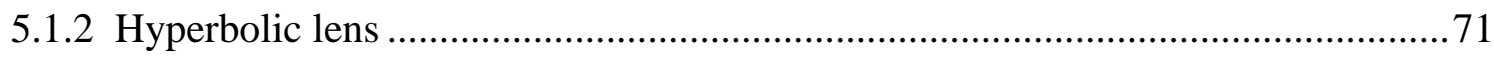

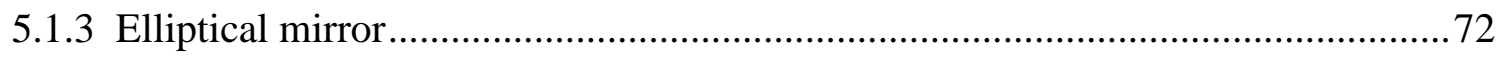

5.2 Numerical examples and validation of the coherent FO........................................... 74

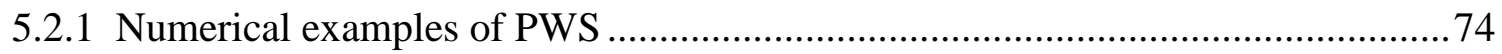

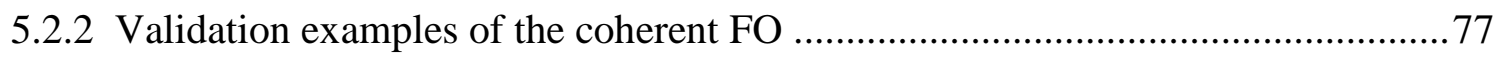

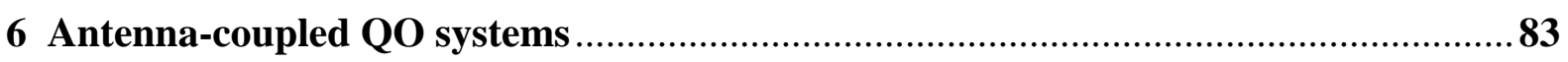

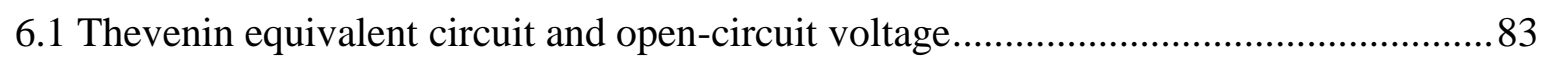

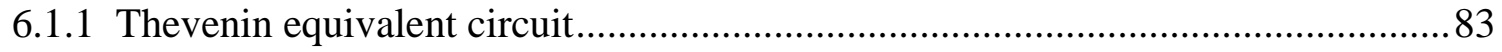

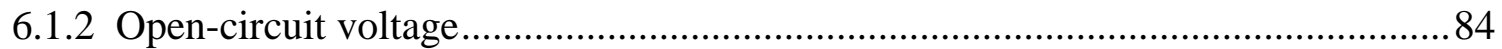

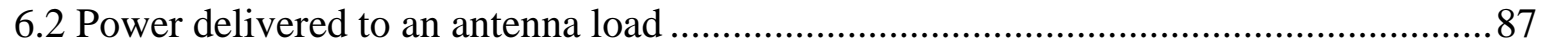

6.3 Performance of antenna-coupled QO systems in reception ......................................90

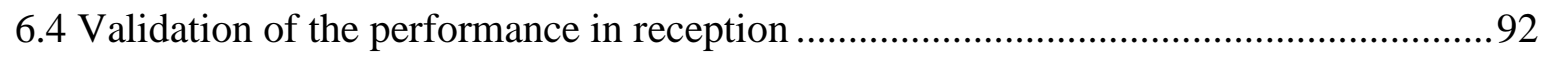

6.4.1 Display a pattern in reception ...................................................................... 92

6.4.2 Examples of validation for all QO components............................................93

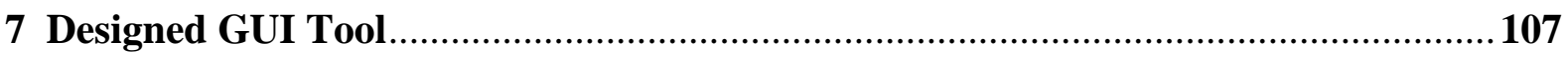

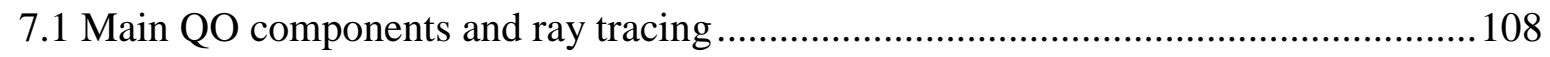

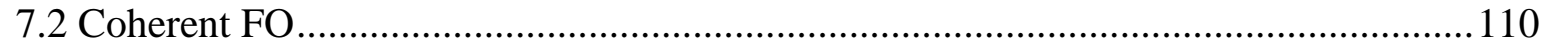

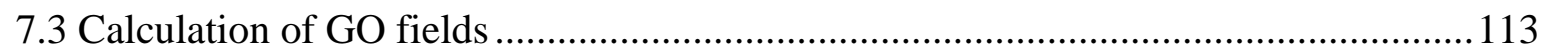

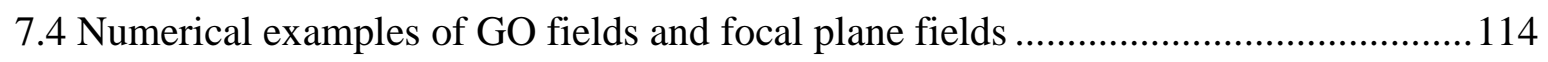

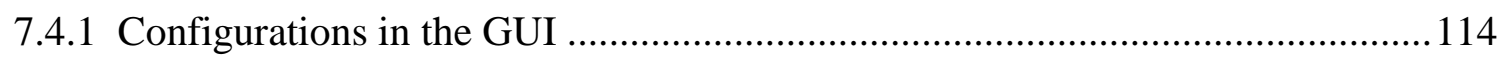

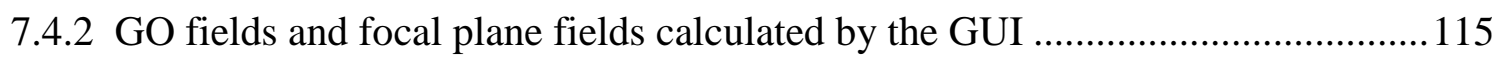

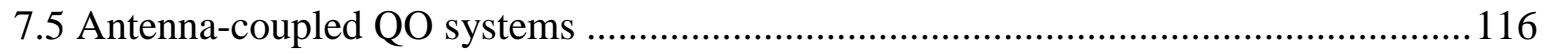

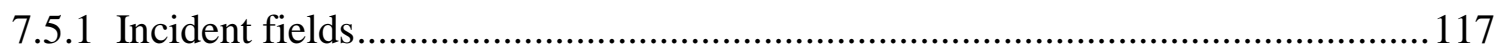

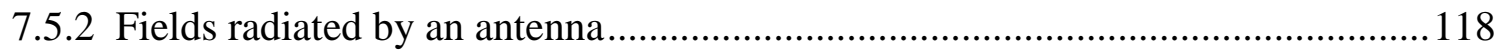

7.5.3 Power delivered to an antenna load and common efficiency terms..................... 122

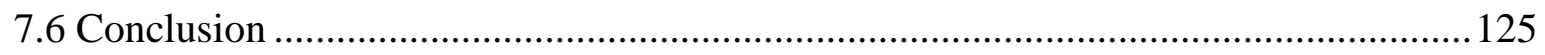

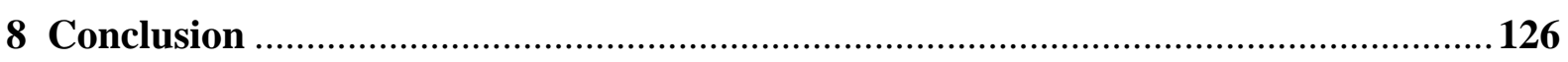

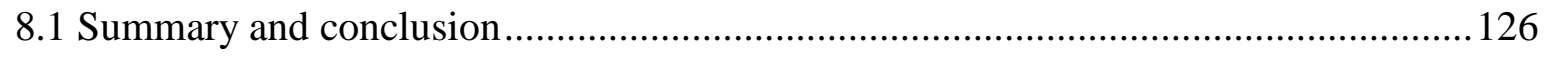

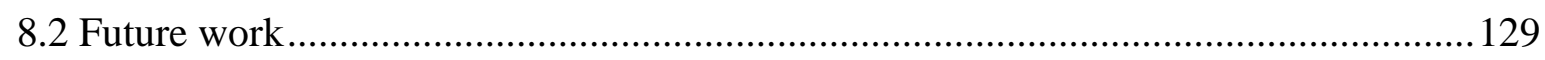

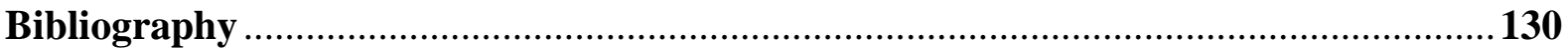




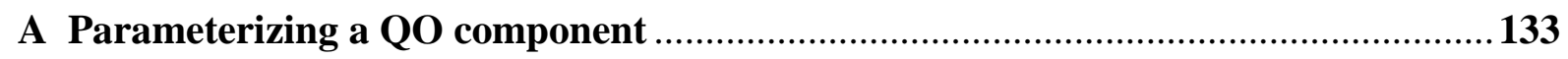

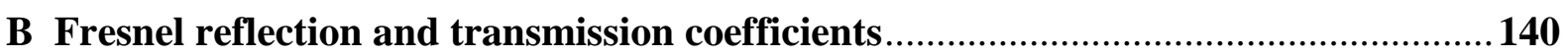

C Additional validation of the implemented PO analysis .............................................. 143

D Re-evaluate the phase approximation for the FO applicability region ......................148

E Characteristic parameters used in GO ray fields....................................................149

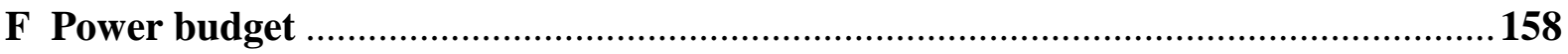

G Spreading factor

H Auxiliary derivations for an elliptical mirror ………….......................................169

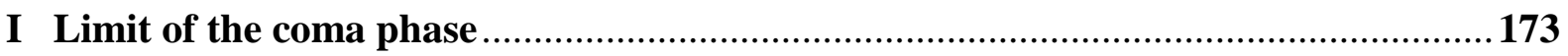

J Additional validation of the derived GO fields ……………………......................177

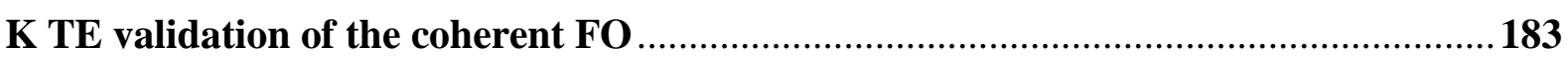




\section{Chapter 1: Introduction}

\subsection{Background}

Systems operating in the Terahertz $(\mathrm{THz})$ frequency domain are becoming more common in recent years in applications such as: Stand-off security monitoring [1, 2], next generation of communication systems [3, 4], far-infrared observatories [5, 6] and etc. In such systems, Quasioptical (QO) components, e.g. reflectors and lenses, are commonly employed to enlarge the directivity of the systems, which leads to higher angular resolution in imaging applications and higher signal to noise ratio in sensing applications. Due to the geometrical properties of QO components, they are employed to radiate highly directive beams when transmitting sources are placed at their foci (transmission scenarios); or focalize incoming fields on their focal planes and increase the sensitivity of the detectors placed at their focal planes (reception scenarios). Here we take a parabolic reflector as an example to better illustrate transmission scenarios (Fig. 1.1a) and reception scenarios (Fig. 1.1b).

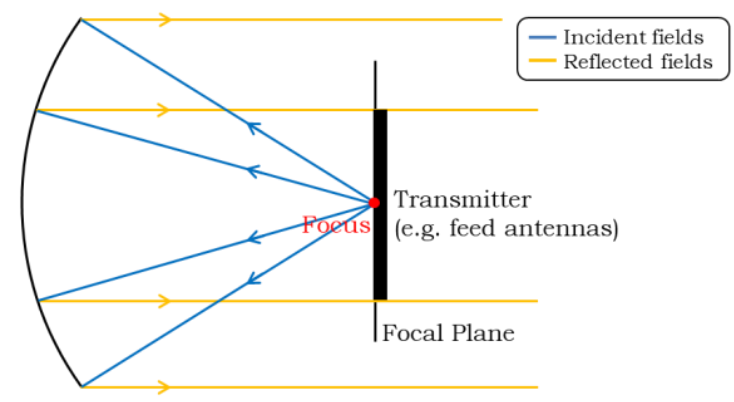

(a)

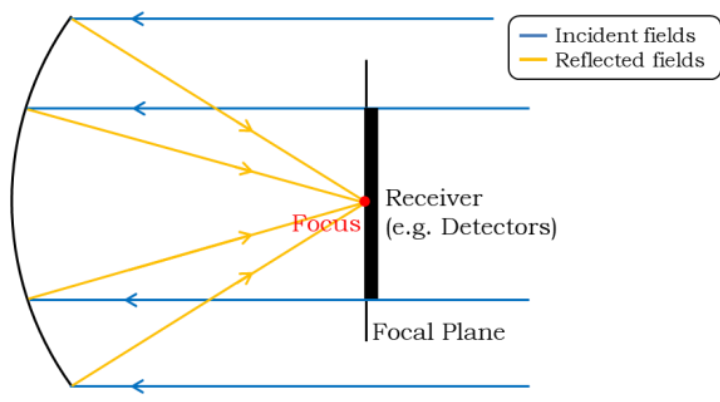

(b)

Figure 1.1: A schematic representation of a parabolic reflector: (a) In transmission scenarios. (b) In reception scenarios.

The analysis of QO systems can be done either in transmission or reception. These analyses are equivalent for single-mode antennas thanks to the reciprocity theorem; whereas it is more convenient to analyze QO systems in reception for multi-mode antennas [7, 8]. Moreover, for absorber-coupled QO systems, they can only be analyzed in reception. In this work, we focus on the analysis in reception because it enables us to combine well-developed methods to analyze the coupling between QO components and antennas or absorbers. Furthermore, the analysis in reception allows us to have a well-defined field coming from the optics that can be used to synthesize the antennas and absorbers integrated with the transmitters and receivers. 
There are several high-frequency techniques that can be used to analyze QO systems, such as: ray tracing, Physical Optics (PO), and Fourier Optics (FO). The ray tracing technique [911] approximates high-frequency electromagnetic (EM) waves as tubes of rays propagating in a homogenous medium, following the laws of reflection and refraction. In such an approach, visualized ray propagation is presented, which is helpful for describing phase aberrations in QO systems. In particular, phase error terms can be included in the analysis by considering the path length traveled by each ray. Therefore, in this work, the ray tracing is used to describe reception scenarios. However, some software, such as ZEMAX [12], cannot provide amplitude information of EM waves. As the result, the ray tracing has a limited use to design antennas or absorbers coupled to QO components. Moreover, the PO method, when the high-frequency approximation is met, can be used to evaluate EM fields scattered by arbitrarily shaped surfaces. This goal is achieved by radiating the equivalent surface currents in absence of the surface $[13,14]$. This technique is well-established and accurate; therefore in this work, it is employed for validation purposes. However, the PO approach is numerically cumbersome and time-consuming, especially for multi-component QO systems. In addition, one cannot easily apply the PO approach to include the coupling between detectors and QO systems.

In order to analyze the coupling between detectors and QO systems in reception. A Fourier Optics approach is proposed in [7, 15] for absorber-coupled QO systems, and in [16] for antenna-coupled systems. In these papers, when a QO system is illuminated by a plane wave, the fields focalized by the QO system on its focal plane can be represented as a summation of plane waves, referred to as plane wave spectrum (PWS). This spectrum is calculated by using the Geometrical Optics (GO) technique [13]. One can link the PWS of the QO system to equivalent Floquet circuits to evaluate the power captured by absorbers placed at the focal plane, as described in [7] and [15]. Moreover, the PWS can be used to analyze the performance of antenna-coupled QO systems. By resorting to the antenna in reception formalism [17], one can use the PWS to estimate the power received by antennas, as described in [16]. Accordingly, compared with the ray tracing and PO approaches, FO based analysis can provide more insight for designing detector-coupled QO systems. However to our knowledge, no universal tools are developed to implement this FO method for variety of QO systems in reception. There are a few codes implementing the FO method [7, 15, 16], but they mainly focus on analyzing parabolic reflectors and elliptical lenses, which constrains design possibilities. Moreover, existing codes do not have user-friendly interfaces, meaning users cannot operate them easily. Accordingly, a MATLAB based graphical user interface (GUI) tool is built in this thesis to aid with analyzing QO systems in reception using the GO/FO method. 


\subsection{Solution proposed in the thesis}

This work proposes a GUI tool for analyzing QO systems in reception using the GO/FO methodology. To improve design possibilities, five widely used QO components are discussed, namely parabolic reflectors, elliptical lenses, hemispherical lenses, hyperbolic lenses, and elliptical mirrors. In addition, the tool takes into account the presence of a matching layer, which is an essential element for designing dielectric lenses. An in-house ray tracing code is developed to describe and visualize reception scenarios for all QO components. Users can apply this ray tracing to preliminarily analyze QO systems by considering phase aberrations. Moreover, a PO code is implemented to evaluate the fields focalized by a QO component on its focal plane. These focal plane fields can be used as references for later-stage validation. The core code is the GO/FO analysis code. It calculates the PWS of the focalized fields by using the fields evaluated on an equivalent FO sphere ([7, 15]) centered at the focus of a QO component. Furthermore, it uses the PWS to estimate the power received by an antenna placed at the focal plane of the QO component, by resorting to the antenna in reception formalism [17]. Accordingly, this GUI tool represents a GO/FO based tool that can be used to analyze and design antenna-coupled QO systems in reception. 


\subsection{Thesis outline}

The thesis is structured as follows. It consists of eight chapters. The second chapter describes the most widely used QO components analyzed in reception. First, main canonical QO components used in this work are introduced, including their applications and parameterizations. Furthermore, the ray tracing technique is discussed to describe reception scenarios for the QO components. In this section, incident fields are defined, and reflected and transmitted fields are evaluated.

In chapter 3, the FO analysis is explained. The field focalized by a QO component on its focal plane is expressed by a spectral representation with a quadratic phase term. To include this phase term in the spectrum, the coherent FO method is investigated.

Chapter 4 focuses on evaluating the fields scattered by a QO component and propagating these fields to the corresponding FO sphere by resorting to the GO technique. These scattered fields are called GO fields and they are the key elements in calculating the coherent FO spectra.

In chapter 5, numerical examples and validation of GO fields and PWS are shown. In chapter 6, antenna-coupled QO systems are analyzed in reception scenarios. To do so, the Thevenin equivalent circuit is introduced to represent a system in reception. By calculating the open-circuit voltage generator in this circuit, the power delivered to the antenna can be evaluated. In addition, the performance of antenna-coupled QO systems is validated by CST and GRASP full-wave simulation software.

In chapter 7, the developed GUI tool is described. The implementations of the ray tracing, the $\mathrm{PO}$, and the GO/FO method are explained. The procedures to obtain coherent FO spectra and to analyze antenna-coupled QO systems are described. To conclude, chapter 8 summarizes main points discussed in the thesis, and sets goals for the future research related to the work done in this thesis. 


\section{Chapter 2: Main canonical QO components}

QO components are important in $\mathrm{THz}$ systems since they can enlarge the directivity of the systems significantly; and in this thesis, they are analyzed in reception. In this chapter, we mainly discuss two things: One is to introduce the QO components used in this thesis; The other is to describe reception scenarios for these QO components.

In section 2.1, the most widely used QO components are discussed, namely parabolic reflectors, elliptical lenses, hemispherical lenses, hyperbolic lenses, and elliptical mirrors. We first introduce some practical applications for each QO component, and then parameterize the geometry of each component by defining its truncation angle, f-number, radial distance, and normal vector. In section 2.2, a reception scenario, where a QO component is illuminated by a certain incident wave, is described by using the ray tracing technique. We discuss incident fields used in this work, analyze their propagation in reflection and transmission problems, and finally show some examples of ray propagation.

\subsection{Canonical QO components}

In this section, we mainly introduce five canonical QO components by discussing their applications in subsection 2.1.1 and parameterizations in subsection 2.1.2 and 2.1.3.

\subsubsection{Applications of different $Q O$ components}

\section{- Parabolic reflector}

A parabolic reflector in reception, as shown in Fig. 2.1, can focalize incident fields at its focal plane and achieve highly directive beams. While in transmission, when a source with a spherical wave front is placed at the focus, by using Snell's law, the reflected fields will be parallel to each other, which translates to highly directive far-field pattern. This high-directivity property makes parabolic reflectors practical in many applications: In astronomical observations, a parabolic reflector acts as a telescope; for instance, ALMA Radio-Telescope for submillimeter observations [18] and Cassini-Huygens Mission for Saturn observation [19]. In telecommunications, it is an important component in satellite and broadcasting communications. Moreover, it can be used in civilian and military security imaging systems [2]. However, in an on-axis configuration, it suffers from a blockage problem, which reduces the aperture efficiency of the system. This issue is illustrated in Fig. 2.1 where a receiver is 
placed at the focal plane of the reflector. As it can be seen, the receiver is placed between the reflector and its far-field region. Therefore, the incident fields also pass the same focal plane, meaning this part of the fields is blocked and wasted. To mitigate this issue, Cassegrain telescope systems [20] and off-axis reflectors are designed. Even so the main properties of the focal plane fields can be understood from a symmetric reflector because most of the dual reflector systems can be modelled by a single paraboloid [21].

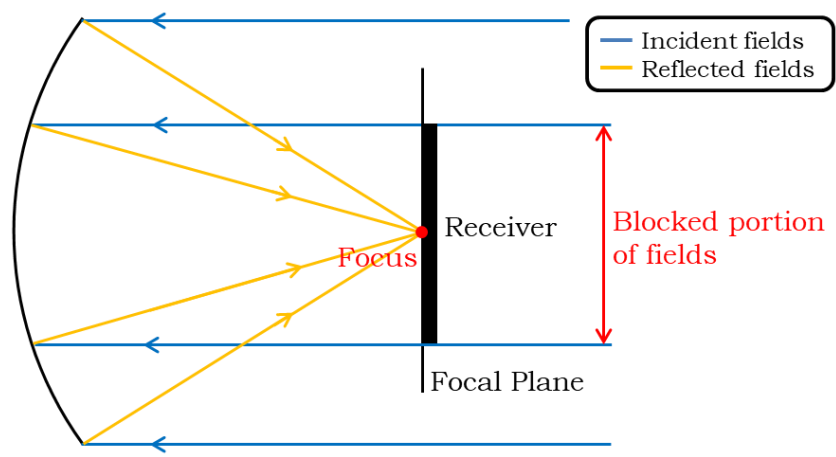

Figure 2.1: A schematic to illustrate wave propagation for a parabolic reflector in reception.

\section{- Elliptical lens}

Integrated antennas are widely used in THz applications. However, since dielectric slabs in integrated chips are electrically thick in $\mathrm{THz}$ frequencies, antennas always suffer from surface waves propagating in substrates, which significantly decreases radiation efficiency [22]. A high-frequency solution to eliminate surface waves is to place a dielectric lens on the top of the antenna. In such a structure, the antenna will radiate most of its power towards the lens [23, 24]. An elliptical surface is always preferred to be used as the dielectric lens since it can radiate directive beams: It can transmit parallel waves when a spherical source is placed at its lower focus (Fig. 2.2), which leads to directive pattern. In practice, elliptical silicon $\left(\varepsilon_{r}=11.9\right)$ lenses are widely used in imaging systems. For example, for space-based observations, a kilopixel imaging system is proposed in [6]. In this system, an array of elliptical silicon lenses is aligned to a detector array that consists of kilo-pixel antenna-coupled MKIDs (Microwave kinetic inductance detectors). 


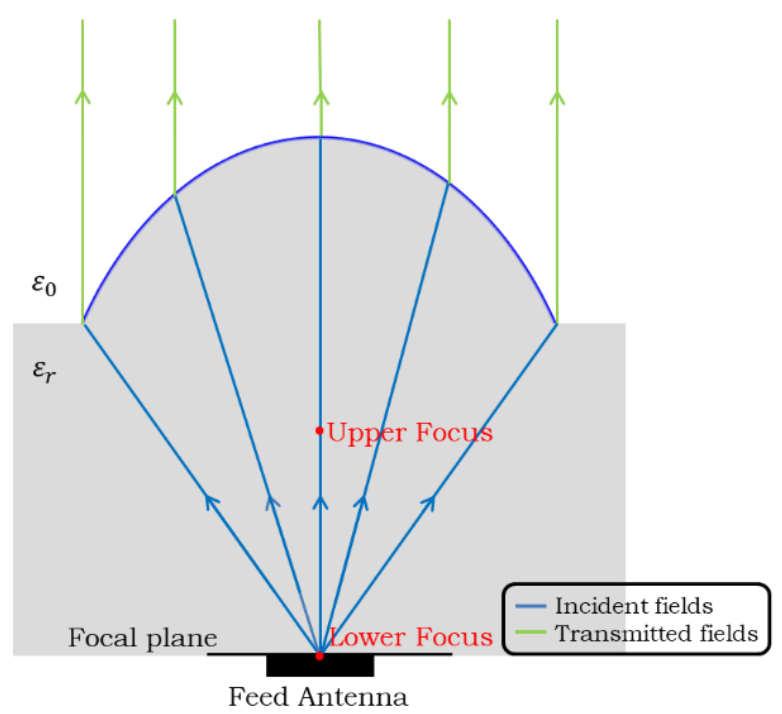

Figure 2.2: A schematic to illustrate wave propagation for an elliptical lens in transmission.

\section{- Hemispherical lens}

Elliptical lenses have very good performance; however, fabricating elliptical surface in dielectric is complicated. Therefore, in practice, one can use an extended hemispherical lens as a substitution to synthesize a true elliptical lens in the case of silicon, as shown in Fig. 2.3. By changing the extension length $L$, one can approximate an elliptical surface by a hemispherical one with reasonable accuracy [24]. In practical applications, for instance, in a large-format imaging system used for cosmic observations, extended hemispherical lenses are integrated with antenna-coupled MKIDs [25].

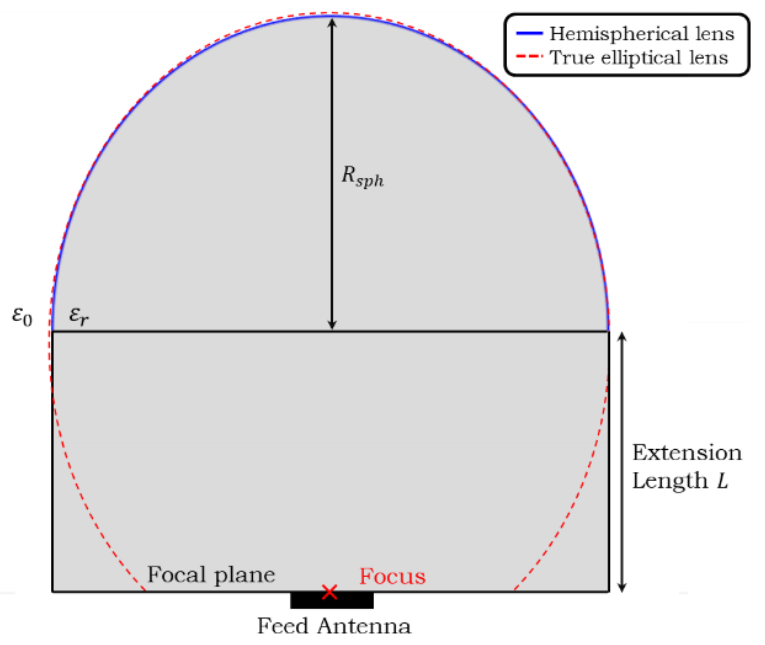

Figure 2.3: Geometry of a synthesized elliptical lens from an extended hemispherical lens, compared with a true elliptical lens in case of silicon. 


\section{- Hyperbolic lens}

In this thesis, we discuss a planar hyperbolic dielectric $\left(\varepsilon_{r}\right)$ lens, i.e. one side is planar and the other side is hyperbolic, as depicted in Fig. 2.4. As it can be seen, this type of hyperbolic lens can focalize the parallel incident fields at its focus without any aberration. It is worth noting that the incident fields and the focal plane are at different sides of the hyperbolic lens. Therefore, there is no blockage problem and the size of the receiver is not limited by the blockage, which are advantages compared with a parabolic reflector. In practical applications, planar hyperbolic lenses can be used for high-resolution far-field imaging $[26,27]$ and $\mathrm{THz}$ spectroscopy systems [28].

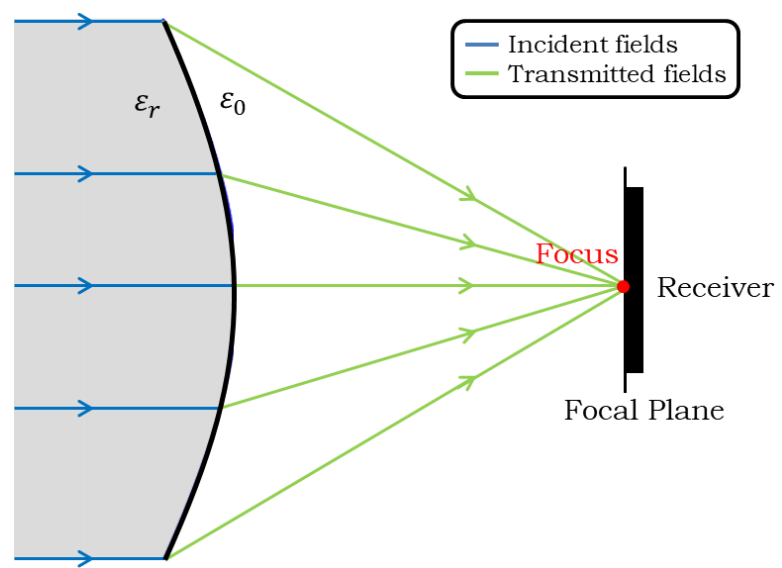

Figure 2.4: A schematic to illustrate wave propagation for a planar hyperbolic lens in reception.

\section{- Elliptical mirror}

An elliptical mirror has two foci, $F_{1}$ and $F_{2}$, as shown in Fig. 2.5. When a point source is placed at one of the foci, for example $F_{2}$, the reflected fields will be focalized at the other focus, $F_{1}$. Due to this property, elliptical mirrors are commonly used in illumination engineering [29, 30]: They are able to collect the light of a source emitted from one focus at the other focus. Moreover, in near-field applications, elliptical reflectors can be used in near-field communication systems [31] and near-field imaging systems, e.g. the detection of breast cancer [32] and THz imaging Radar [33]. 


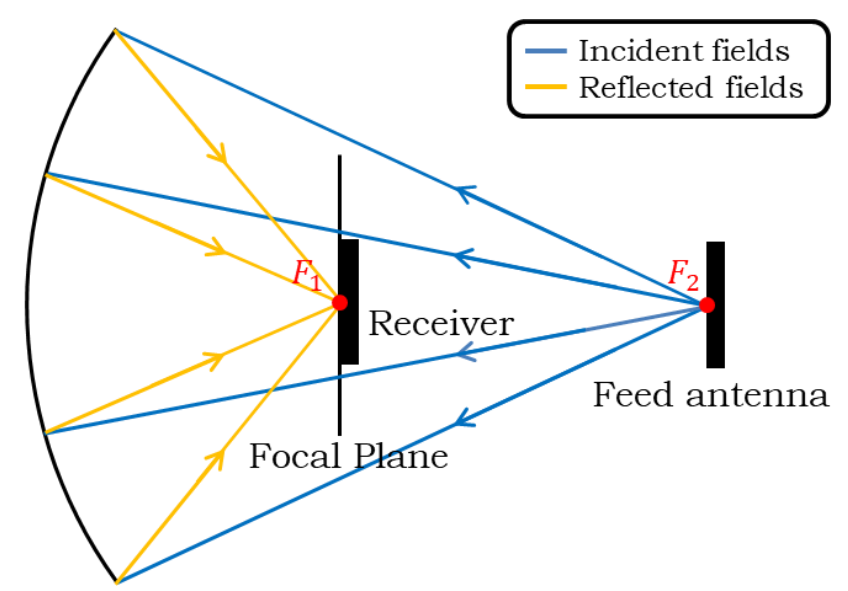

Figure 2.5: A schematic to illustrate wave propagation for an elliptical mirror.

\subsubsection{Parameterizing a generic surface}

Before introducing a specific QO component, here a generic surface is parameterized. To do so, two parameters are considered: Parameterization variables and normal vectors.

\subsubsection{Parameterization variables}

When defining a surface, one should perform a mapping from a 2D domain $(m, n)$ to a 3D domain $(x, y, z)$. This mapping can be expressed as:

$$
(m, n) \rightarrow\left\{\begin{array}{l}
x=f_{x}(m, n) \\
y=f_{y}(m, n) \\
z=f_{z}(m, n)
\end{array}\right.
$$

In this thesis, two types of 2D domains (variables), $(\theta, \phi)$ and $(u, v)$, and one 3D domain, cartesian coordinate $(x, y, z)$, are used. We start with an arbitrary surface. As it can be seen in Fig. 2.6, $Q$ is a point on a generic surface $S$ with the radial distance $r(\theta)$. $\hat{n}_{Q}$ is the normal vector of $S$ and $\theta_{0}$ is the subtended rim angle where we truncate the surface.

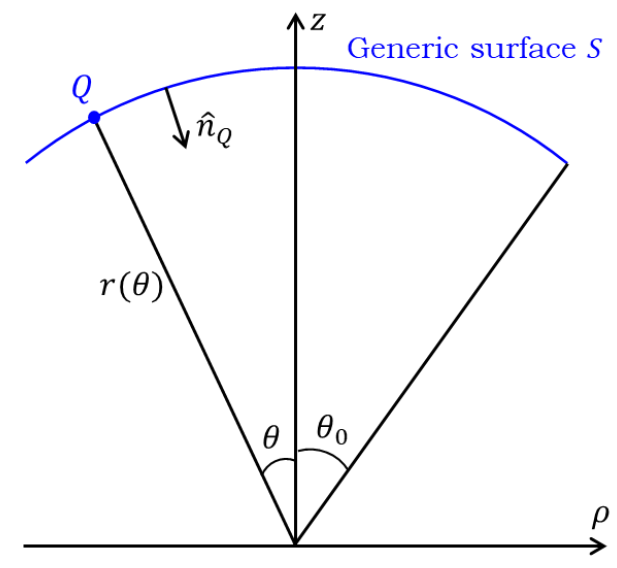

Figure 2.6: Sketch of a generic surface $S$. 


\section{- Parameterization using $(\theta, \phi)$ variables}

In this case, the mapping in Eq. (2.1) becomes:

$$
(\theta, \phi) \rightarrow\left\{\begin{array}{l}
x=f_{x}(\theta, \phi) \\
y=f_{y}(\theta, \phi) \\
z=f_{z}(\theta, \phi)
\end{array}\right.
$$

Therefore, the point $Q$ can be parameterized as follows:

$$
\left\{\begin{array}{l}
Q_{x}=r(\theta) \sin \theta \cos \phi \\
Q_{y}=r(\theta) \sin \theta \sin \phi \\
Q_{z}=r(\theta) \cos \theta
\end{array}\right.
$$

\section{- Parameterization using $(u, v)$ variables}

The $(\theta, \phi)$ variables are easy to define; however, using them would lead to a problem that the surface is sampled denser at the center and less at the edge (illustrated clearly in Appendix A.1). This non-uniform sampling means that the fields on the surface could require a large number of points to converge. Therefore, we introduce another set of variables that performs uniform sampling, the $(u, v)$ variables, which are obtained as follows:

$$
\left\{\begin{array}{l}
u=\sin \theta \cos \phi \\
v=\sin \theta \sin \phi
\end{array}\right.
$$

By using these variables, the surface can be parameterized as:

$$
\left\{\begin{array}{l}
Q_{x}=r(u, v) u \\
Q_{y}=r(u, v) v \\
Q_{z}=r(u, v) \sqrt{1-\left(u^{2}+v^{2}\right)}
\end{array}\right.
$$

Since $(\theta, \phi)$ and $(u, v)$ can be mutually transformed by using Eq. (2.4), in the following parts of this thesis, for simplicity, expressions in $(\theta, \phi)$ are reported for most cases.

\subsubsection{Normal vectors}

Normal vectors are important when calculating the Jacobian of a surface or applying Snell's law. Therefore, a generic expression for normal vectors of a surface is discussed. After parameterizing the surface $S$, one can calculate the normal vector $\hat{n}_{Q}$ at each point on the surface as:

$$
\hat{n}_{Q}= \pm \frac{\frac{\partial \vec{Q}}{\partial \theta / u} \times \frac{\partial \vec{Q}}{\partial \phi / v}}{\left|\frac{\partial \vec{Q}}{\partial \theta / u} \times \frac{\partial \vec{Q}}{\partial \phi / v}\right|}
$$

where $\vec{Q}=Q_{x} \hat{x}+Q_{y} \hat{y}+Q_{z} \hat{z}, \frac{\partial \vec{Q}}{\partial \theta / u}$ and $\frac{\partial \vec{Q}}{\partial \phi / v}$ are partial derivatives of the surface with respect to $(\theta, \phi)$ or $(u, v)$, which are explicitly calculated in Appendix A.2. The sign " \pm " in Eq. (2.6) 
indicates the direction of the normal vector: " + " means $\hat{n}_{Q}$ points towards the $+z$ direction, while “-” means the $-z$ direction. In this thesis, the selection of the sign depends on the direction of the incident field. When the incident field propagates towards $+z$ direction, the sign is " - "; while for $-z$ propagation, the sign is " + ".

\subsubsection{Parameterizing a specific QO component}

In this subsection, we consider parameterizing a QO component, with a specific truncation angle (derived in Appendix A.3), f-number, radial distance, and normal vector.

\section{- Parabolic reflector}

Fig. 2.7 shows the 2D geometry (front view) of a parabolic reflector. The reflector is centered at its focus $O$, with the diameter of $D_{r}$ and the focal distance of $f$. The f-number is defined as $f_{\#}^{l}=f / D_{r}$. It is worth noting that in reception, the observation point is also located at $O$, i.e. we place a receiver at $O$. The radial distance, $r(\theta)$, can be expressed as:

$$
r(\theta)=\frac{2 f}{1+\cos \theta}
$$

We can also calculate the normal vector of the surface, $\hat{n}_{r}$, by using Eq. (2.6) (extended calculation in Appendix A.2):

$$
\hat{n}_{r}=-\sqrt{\frac{1-\cos \theta}{2}} \hat{\rho}-\sqrt{\frac{1+\cos \theta}{2}} \hat{z}
$$

\section{- Elliptical lens}

An ideal elliptical lens should achieve directive patterns. To achieve this in transmission, one needs to ensure the fields on the lens equivalent aperture have constant phase. The resulting lens should have the eccentricity of $e=1 / \sqrt{\varepsilon_{r}}$, where $\varepsilon_{r}$ is the relative permittivity of the dielectric; and an antenna should be placed at its lower focus. This configuration is shown in Fig. 2.8 that we move the center of the coordinate system (observation point) to the lower focus of the ellipse, $\mathrm{O}_{2}$. The distance from $\mathrm{O}_{2}$ to the apex is $a+c$, where $a$ is the semi-major axis and $c$ is the focal distance. The $\mathrm{f}$-number is defined as $f_{\#}^{r}=R_{l} / D_{l}$, where $R_{l}$ is the rim distance (Appendix A.3). For the surface of the lens, the radial distance, $r(\theta)$, can be expressed as:

$$
r(\theta)=a \frac{1-e^{2}}{1-e \cos \theta}
$$


where $e=c / a=1 / \sqrt{\varepsilon_{r}}$ is the eccentricity of the ellipse. The normal vector of the surface, $\hat{n}_{l}$, is obtained as follows:

$$
\hat{n}_{l}=\frac{\sin \theta}{\sqrt{1+e^{2}-2 e \cos \theta}} \hat{\rho}+\frac{\cos \theta-e}{\sqrt{1+e^{2}-2 e \cos \theta}} \hat{z}
$$

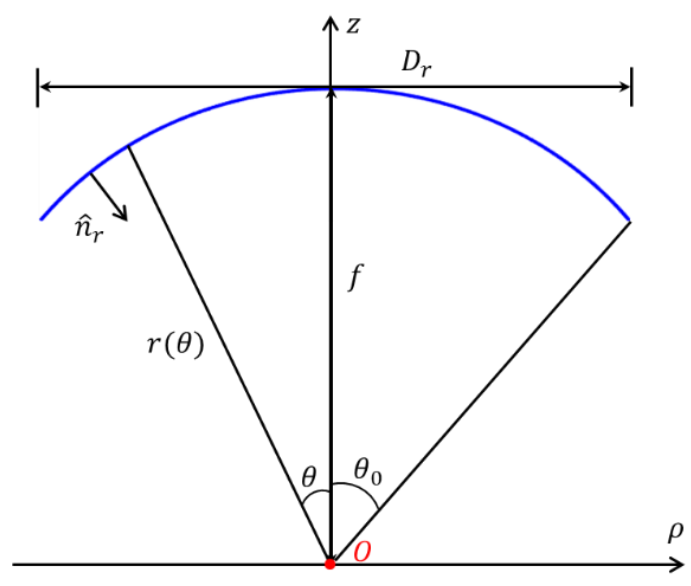

Figure 2.7: 2D geometry of a parabolic reflector.

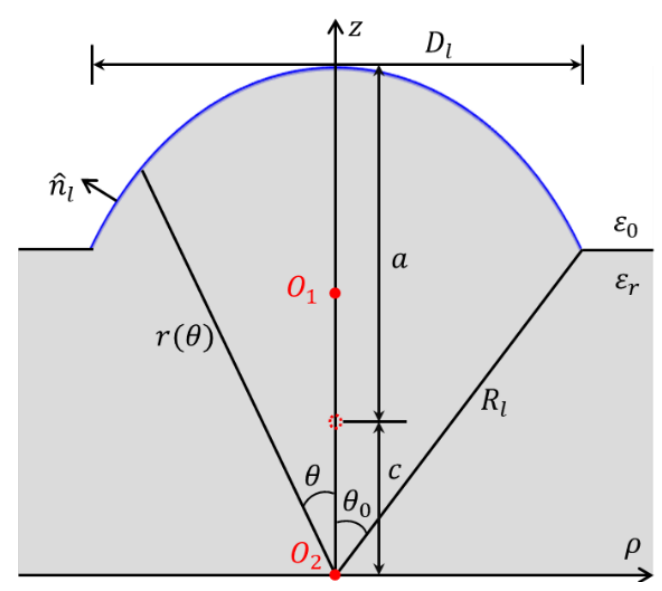

Figure 2.8: 2D geometry of an elliptical lens.

\section{- Hemispherical lens}

A hemispherical lens (Fig. 2.9) consists of a hemisphere with the radius of $R_{s p h}$ and an extended base with the length of $L$. The center of the hemisphere is represented by $O^{\prime}$, while the center of the coordinate system (observation point) is $O$. The f-number is defined as $f_{\#}^{h l}=$ $R_{l} / D_{h l}$, where $R_{l}$ is the rim distance calculated in Appendix A.3. The radial distance, $r(\theta)$, can be expressed as:

$$
r(\theta)=L \cos \theta+\sqrt{R_{s p h}^{2}-L^{2} \sin ^{2} \theta}
$$

Since $r(\theta)$ has a complicated expression, the normal vector of the surface, $\hat{n}_{h l}$, is calculated numerically by using Eq. (2.6).

In practical design, two types of hemispherical lenses are commonly used: One is the configuration introduced in [24]. If the extension length is selected as $L / R_{s p h}=0.32 \sim 0.35$, an elliptical lens can be approximated by a hemispherical lens with reasonable accuracy. The other configuration is called hyperhemispherical lens $[24,34]$. The extension is designed to be $L=R_{s p h} / \sqrt{\varepsilon_{r}}$, with which incident spherical waves can be perfectly focalized by the lens on its focal plane. 


\section{- Hyperbolic lens}

In this work we discuss a planar hyperbolic lens, as shown in Fig. 2.10. An ideal hyperbolic lens should achieve directive patterns. To achieve this in transmission, one needs to ensure the fields on the lens equivalent aperture have constant phase. The resulting lens should have the eccentricity of $e=\sqrt{\varepsilon_{r}}$, where $\varepsilon_{r}$ is the relative permittivity of the dielectric; and an antenna should be placed at its lower focus. In Fig. 2.10, we move the center of the coordinate system to the lower focus $O$ and define the focal distance as $f=a+c$, where $a$ is the semi-major axis and $c$ is half of the distance between two foci. The f-number is defined as $f_{\#}^{h}=f / D_{h}$. The radial distance, $r(\theta)$, can be expressed as:

$$
r(\theta)=-\frac{b^{2} / a}{1-e \cos \theta}
$$

where $b=\sqrt{c^{2}-a^{2}}$ and $e=c / a=\sqrt{\varepsilon_{r}}$ is the eccentricity of the hyperbola. We can also calculate the normal vector, $\hat{n}_{h}$ :

$$
\hat{n}_{h}=-\frac{\sin \theta}{\sqrt{1+e^{2}-2 e \cos \theta}} \hat{\rho}+\frac{e-\cos \theta}{\sqrt{1+e^{2}-2 e \cos \theta}} \hat{z}
$$

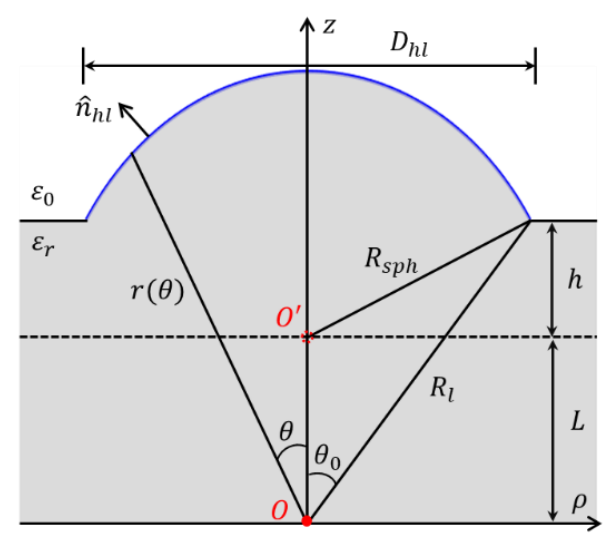

Figure 2.9: 2D geometry of a hemispherical lens.

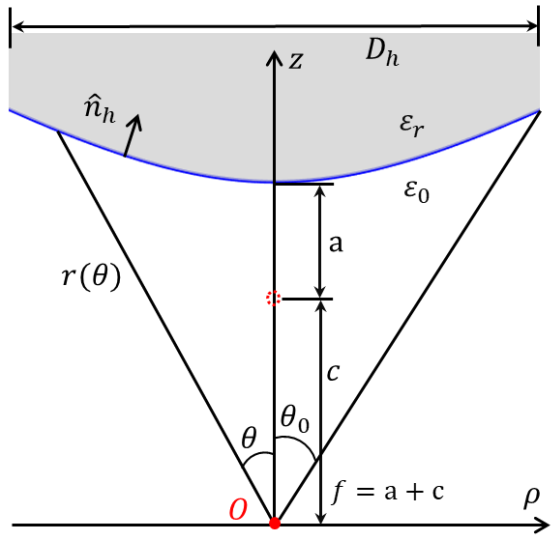

Figure 2.10: 2D geometry of a hyperbolic lens.

\section{- Elliptical mirror}

An elliptical mirror has two foci, $O_{1}$ and $O_{2}$, as shown in Fig. 2.11. When a transmitter is placed at one focus, for example $O_{2}$, a receiver should be placed the other focus, $O_{1}$. Therefore, depending on where we place a receiver (observation point), the surface can be parameterized by $r_{1}\left(\theta_{1}\right)$ or $r_{2}\left(\theta_{2}\right)$. The radial distance, $r_{1}\left(\theta_{1}\right)$ and $r_{2}\left(\theta_{2}\right)$, can be expressed as follows: 


$$
\left\{\begin{array}{l}
r_{1}\left(\theta_{1}\right)=a \frac{1-e^{2}}{1+e \cos \theta_{1}} \\
r_{2}\left(\theta_{2}\right)=a \frac{1-e^{2}}{1-e \cos \theta_{2}}
\end{array}\right.
$$

where $a$ is the semi-major axis and $e=c / a$ is the eccentricity. It can be observed from Fig. 2.11 that $r_{1}\left(\theta_{1}\right)$ and $r_{2}\left(\theta_{2}\right)$ are related to each other as follows:

$$
r_{1}\left(\theta_{1}\right) \cos \theta_{1}+2 c=r_{2}\left(\theta_{2}\right) \cos \theta_{2}
$$

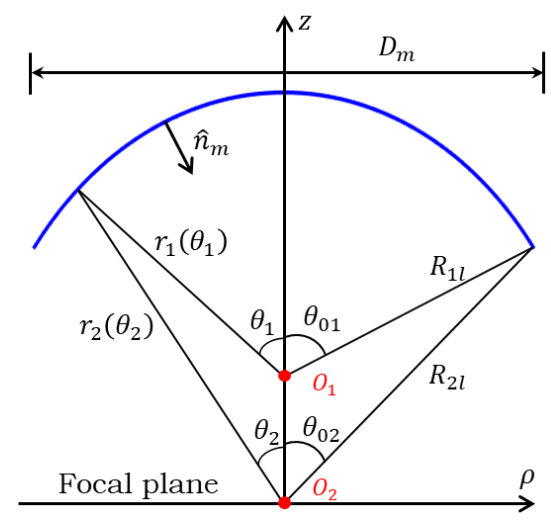

(a)

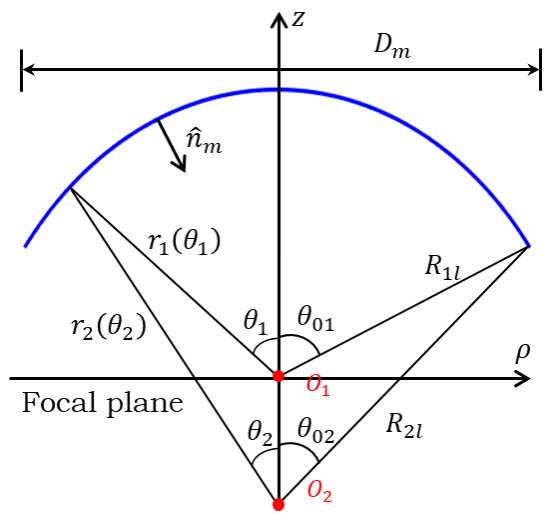

(b)

Figure 2.11: 2D geometry of an elliptical mirror: (a) Observation at lower focus. (b) Observation at upper focus.

\section{Case 1: Observation at lower focus, $\mathrm{O}_{2}$}

In this case, as shown in Fig. 2.11a, the f-number is defined as $f_{\#}^{m}=R_{2 l} / D_{m}$, where $R_{2 l}$ is the rim distance calculated in Appendix A.3. The mirror is parameterized by the radial distance, $r_{2}\left(\theta_{2}\right)$. By solving Eq. (2.15), one can relate $\theta_{1}$ to $\theta_{2}$ :

$$
\theta_{1}=\cos ^{-1} \frac{A_{1} a\left(1-e^{2}\right)-2 c}{2 c e+\left(1-A_{1} e\right) a\left(1-e^{2}\right)}, A_{1}=\frac{\cos \theta_{2}}{1-e \cos \theta_{2}}
$$

The normal vector, $\hat{n}_{m}$, can be expressed as:

$$
\hat{n}_{m}=-\frac{\sin \theta_{2}}{\sqrt{1+e^{2}-2 e \cos \theta_{2}}} \hat{\rho}-\frac{\cos \theta_{2}-e}{\sqrt{1+e^{2}-2 e \cos \theta_{2}}} \hat{z}
$$

\section{Case 2: Observation at upper focus, $O_{1}$}

In this case, as shown in Fig. 2.11b, the f-number is defined as $f_{\#}^{m}=(a-c) / D_{m}$. The mirror is parameterized by the radial distance, $r_{1}\left(\theta_{1}\right)$. By solving Eq. (2.15), one can relate $\theta_{2}$ to $\theta_{1}$ :

$$
\theta_{2}=\cos ^{-1} \frac{A_{2} a\left(1-e^{2}\right)+2 c}{2 c e+\left(1+A_{2} e\right) a\left(1-e^{2}\right)}, A_{2}=\frac{\cos \theta_{1}}{1+e \cos \theta_{1}}
$$


The normal vector, $\hat{n}_{m}$, can be calculated as:

$$
\hat{n}_{m}=-\frac{\sin \theta_{1}}{\sqrt{1+e^{2}+2 e \cos \theta_{1}}} \hat{\rho}-\frac{\cos \theta_{1}+e}{\sqrt{1+e^{2}+2 e \cos \theta_{1}}} \hat{z}
$$

\subsection{Ray tracing technique}

As discussed in [13], for high-frequency scenarios, i.e. the surface of a QO component is large with respect to wavelength, one can approximate EM waves as tubes of rays that propagate from one point to another in a homogenous medium, following the laws of reflection and refraction. By tracing ray propagation, a reception scenario can be well described. One can clearly observe how incident rays are focalized by a QO component in a reflection or transmission problem. Therefore, ray tracing is helpful for describing phase aberrations for QO systems in reception.

In this section, we introduce the ray tracing technique implemented in the tool, which mainly focuses on calculating directions of propagation and polarizations for incident, reflected, and transmitted rays. In subsection 2.2.1, we describe the incident field used in this work. And then in subsection 2.2.2, we discuss how we solve reflection and transmission problems by using Snell's law and boundary conditions. Finally, in subsection 2.2.3, we show some visualized ray tracing plots generated by the ray tracing technique.

\subsubsection{Incident field}

There are two sets of sources used in the thesis: A plane wave and a point source. For an elliptical mirror, we use a point source (Huygens source) to generate incident fields; while for other QO components, since the source is at infinity, we use a plane wave as the incident field.

\subsubsection{Plane wave}

In Fig. 2.12, a plane wave incoming with the skew angle of $\left(\theta_{S}, \phi_{S}\right)$ impinges on a generic surface $S$. We can characterize the incident plane wave at each point $Q$ on the surface, with the position vector $\vec{r}(\theta)$ :

$$
\vec{E}_{i}(\vec{r})=E_{0} e^{-j k\left(\hat{s}_{i} \cdot \vec{r}\right)} \hat{p}_{i}
$$

where $E_{0}$ is the amplitude of the electric field, $k$ is the propagation constant of the medium, $\hat{s}_{i}$ is the propagation unit vector, and $\hat{p}_{i}$ is the polarization of the electric field. The incident magnetic field can be calculated as follows:

$$
\vec{H}_{i}(\vec{r})=\frac{1}{\zeta} \hat{s}_{i} \times \vec{E}_{i}(\vec{r})
$$


where $\zeta$ is the impedance of the medium. In the following sections, unless we address the calculation of magnetic fields, we only introduce electric fields and assume a plane wave relation described in Eq. (2.21) to calculate magnetic fields. The propagation unit vector can be expressed as:

$$
\hat{s}_{i}=\sin \theta_{s} \cos \phi_{s} \hat{x}+\sin \theta_{s} \sin \phi_{s} \hat{y}+\cos \theta_{s} \hat{z}
$$

where $\left(\theta_{s}, \phi_{s}\right)$ is the skew angle of the incident rays. As for the polarization, $\hat{p}_{i}$, it is defined by using the Ludwig-III definition described in [35]: $\hat{p}_{i}$ can be either the reference polarization (Co-Pol.):

$$
\hat{p}_{i, C o}=\sin \phi_{s} \hat{\theta}_{s}+\cos \phi_{s} \hat{\phi}_{s}
$$

or the cross polarization (Cx-Pol.):

$$
\hat{p}_{i, C x}=\cos \phi_{s} \hat{\theta}_{s}-\sin \phi_{s} \hat{\phi}_{s}
$$

where $\hat{\theta}_{S}$ and $\hat{\phi}_{s}$ can be expressed as:

$$
\left\{\begin{array}{l}
\hat{\theta}_{s}=\cos \theta_{s} \cos \phi_{s} \hat{x}+\cos \theta_{s} \sin \phi_{s} \hat{y}-\sin \theta_{s} \hat{z} \\
\hat{\phi}_{s}=-\sin \phi_{s} \hat{x}+\cos \phi_{s} \hat{y}
\end{array}\right.
$$

It is worth noting that when $\phi_{s}=90^{\circ}, \hat{p}_{i, C o}=\hat{\theta}_{s}$, which is referred to as the TM polarization; and when $\phi_{s}=0^{\circ}, \hat{p}_{i, C o}=\hat{\phi}_{s}$, which is referred to as the TE polarization.

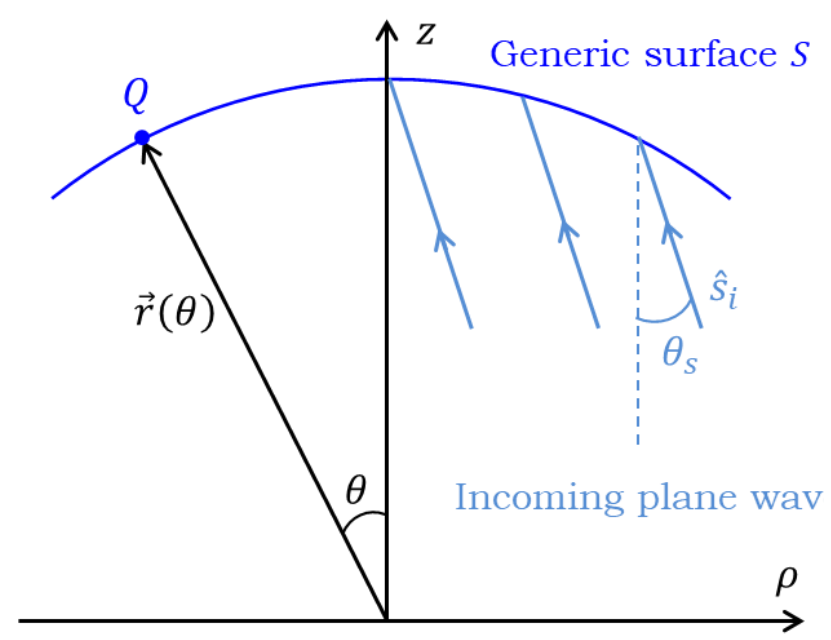

Figure 2.12: A plane wave incoming with the skew angle of $\left(\theta_{s}, \phi_{s}\right)$. 


\subsubsection{Point source}

In the case of an elliptical mirror, we want to illuminate its surface with a spherical wave. By emitting fields with spherical wave fronts at one focus, one can obtain reflected spherical wave fonts focalized at the other focus. To do so, a simple case is to adopt a point source as the testing source, and put it at one of the foci of the ellipse. In this thesis, we use a Huygens source as the point source since a symmetric far-field pattern can be achieved by this source [36]; whereas an elementary electric dipole has a $\phi$-related pattern. A Huygens source consists of two elementary dipoles, one is electric and the other is magnetic, as shown in the inset in Fig. 2.13. The magnetic dipole, $\vec{M}$, should have the following relation with the electric dipole, $\vec{J}$ :

$$
\left\{\begin{array}{c}
|\vec{M}|=\zeta|\vec{J}| \\
\vec{M} \cdot \vec{J}=0 \\
\vec{J} \times \vec{M}=\hat{z}
\end{array}\right.
$$

where $\zeta$ is the impedance of the medium. It can also be seen in Fig. 2.13 that we define the angle between the electric dipole and the $x$ axis as the orientation angle, $\gamma$. When $\gamma=0^{\circ}, \vec{J}$ is oriented along $\hat{x}$ direction and the Huygens source is x-polarized (Co-Pol.); while when $\gamma=$ $90^{\circ}, \vec{J}$ is oriented along $\hat{y}$ direction and the Huygens source is y-polarized (Cx-Pol.).

When we put the source at one of the foci of the ellipse (broadside incidence), the far field generated by the Huygens source can be described as follows:

$$
\vec{E}_{i}=\left(E_{i}^{\perp} \hat{\phi}+E_{i}^{\|} \hat{\theta}\right) \frac{e^{-j k r}}{r}
$$

where $r$ is the radial distance from the focus to the surface, $E_{i}^{\|}$is the parallel (TM) component, and $E_{i}^{\perp}$ is the perpendicular (TE) component. When we move the source within one focal plane with a distance, $\vec{\rho}_{s}$, for instance, within the upper focal plane as depicted in Fig. 2.13, we calculate the far field numerically by using the far-field approximation of Green's function. Here we define the skew angle of the incident rays, i.e. $\left(\theta_{s}, \phi_{s}\right)$, as follows:

$$
\left\{\begin{array}{l}
\theta_{s}=\tan ^{-1} \frac{\left|\vec{\rho}_{s}\right|}{a \pm c} \\
\phi_{s}=\tan ^{-1} \frac{\left|\vec{\rho}_{s, x}\right|}{\left|\vec{\rho}_{s, y}\right|}
\end{array}\right.
$$

where $\vec{\rho}_{s}=\vec{\rho}_{s, x}+\vec{\rho}_{s, y}, \vec{\rho}_{s, x}$ and $\vec{\rho}_{s, y}$ are the $x$-and $y$-oriented displacement, respectively. The sign " \pm " in Eq. (2.28) depends on where we put the source: "-" for the case of upper focal plane, while "+" for the case of lower focal plane. 


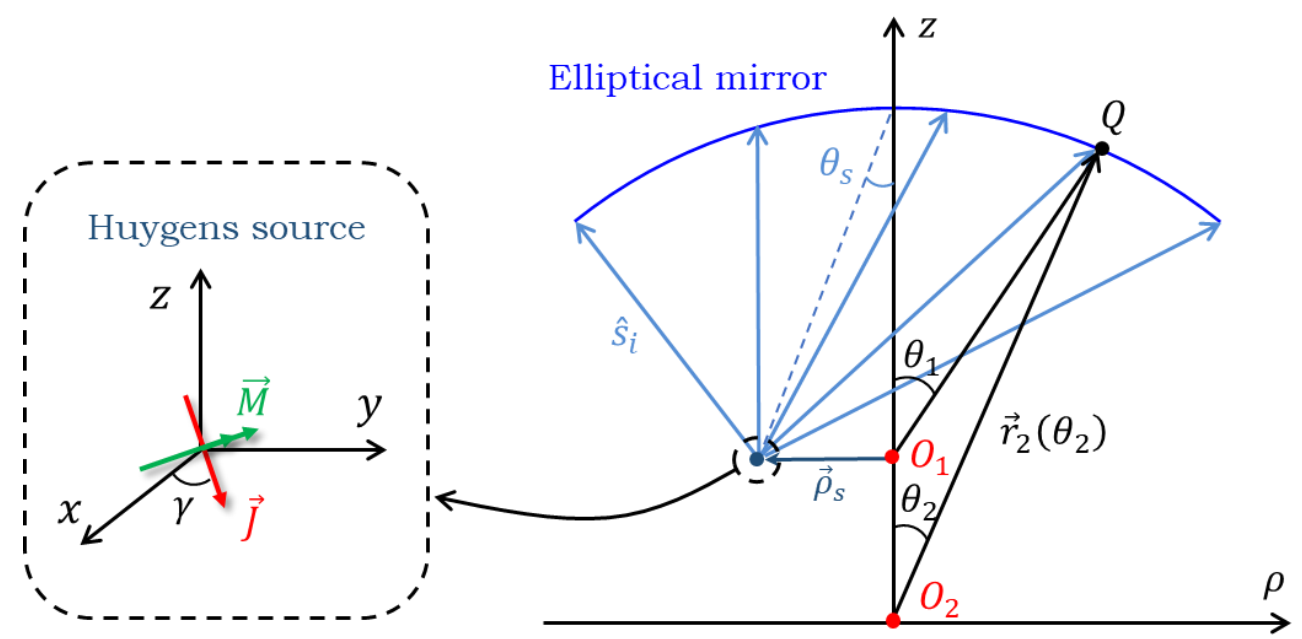

Figure 2.13: A Huygens source placed at the upper focal plane of an elliptical mirror, with a displacement $\vec{\rho}_{s}$.

\subsubsection{Reflection and transmission at a flat interface}

In this subsection, we focus on representing reflected and transmitted fields at a flat interface, by taking propagation unit vectors, polarizations, and Fresnel reflection and transmission coefficients into account. First, we discuss a case that involves two mediums. Furthermore, we explore a case with the implementation of a matching layer.

\subsubsection{Without a matching layer}

To solve a reflection and transmission problem, we observe a point $Q$ on a surface locally, as depicted in Fig. 2.14. Since we are in the high-frequency domain, a surface can be approximated as a locally infinite flat plane between two mediums, I and II, with relative permittivity $\varepsilon_{r 1}$ and $\varepsilon_{r 2}$, respectively. $\hat{n}$ is the normal vector at $Q$ pointing from II to I.

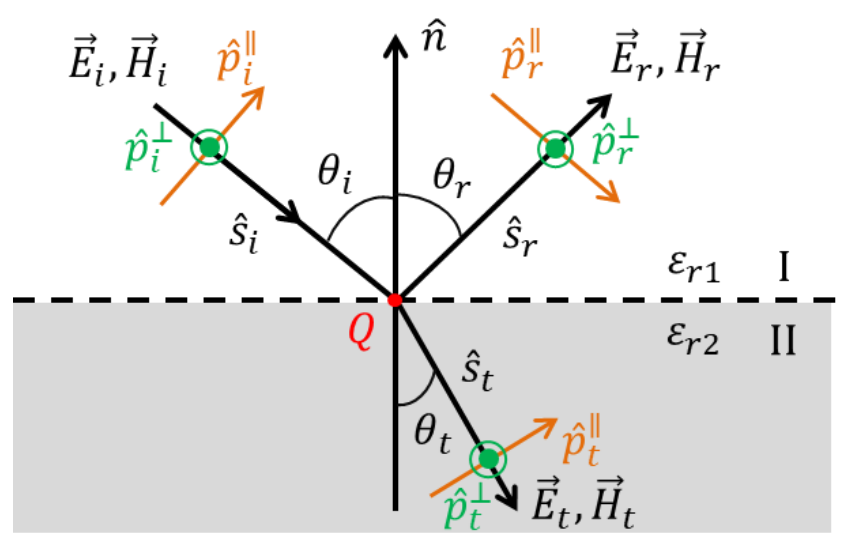

Figure 2.14: Flat interface between medium I and II, illuminated by an incident ray. 
We can describe the scenario in Fig. 2.14 as: An incident ray arrives to a point $Q$ with the propagation unit vector $\hat{s}_{i}$ and the incident angle $\theta_{i}$. Part of this field is reflected back to medium I with the direction $\hat{s}_{r}$ and the reflected angle $\theta_{r}$; while part of the field is transmitted into medium II with the direction $\hat{s}_{t}$ and the transmitted angle $\theta_{t}$. All electric fields $\left(\vec{E}_{i}, \vec{E}_{r}\right.$, and $\vec{E}_{t}$ ) are divided into perpendicular (TE) and parallel (TM) components, with the polarization unit vectors $\hat{p}_{i / r / t}^{\perp / \|}$.

\section{- Propagation unit vectors}

We use Snell's law to calculate the propagation unit vectors: $\hat{s}_{r}$ and $\hat{s}_{t}$. Snell's law is known as:

$$
\left\{\begin{array}{l}
\theta_{i}=\theta_{r} \\
n_{1} \sin \theta_{i}=n_{2} \sin \theta_{t}
\end{array}\right.
$$

where $n_{1 / 2}=\sqrt{\varepsilon_{r 1 / 2}}$ is the refractive index of the medium. However, with this representation, obtaining $\hat{s}_{r}$ and $\hat{s}_{t}$ from $\hat{s}_{i}$ is not straight forward. By considering that $\sin \theta=\hat{s} \times \hat{n}$ and $\cos \theta=\hat{s} \cdot( \pm \hat{n})$, we can represent Eq. (2.29) in a vectorial form:

$$
\left\{\begin{array}{l}
\hat{s}_{r}=\hat{s}_{i}-2\left(\hat{s}_{i} \cdot \hat{n}\right) \hat{n} \\
\hat{s}_{t}=\sqrt{\frac{\varepsilon_{r 1}}{\varepsilon_{r 2}}} \hat{s}_{i}-\left[\sqrt{\frac{\varepsilon_{r 1}}{\varepsilon_{r 2}}}\left(\hat{s}_{i} \cdot \hat{n}\right)+\sqrt{\frac{1}{\varepsilon_{r 2}}} \sqrt{\varepsilon_{r 2}-\varepsilon_{r 1}\left(1-\left(\hat{s}_{i} \cdot \hat{n}\right)^{2}\right)}\right] \hat{n}
\end{array}\right.
$$

\section{- Polarizations}

Since we decompose the electric field into TE and TM components, we need to define their corresponding polarization unit vectors, $\hat{p}_{i / r / t}^{\perp / \|}$. If we define them using the directions depicted in Fig. 2.14, the polarizations of the incident and the transmitted rays can be calculated by using the same expression:

$$
\left\{\begin{array}{l}
\hat{p}_{i / t}^{\perp}=\frac{\hat{s}_{i / t} \times \hat{n}}{\left|\hat{s}_{i / t} \times \hat{n}\right|} \\
\hat{p}_{i / t}^{\|}=\hat{p}_{i / t}^{\perp} \times \hat{s}_{i / t}
\end{array}\right.
$$

while for the reflected ray:

$$
\left\{\begin{array}{l}
\hat{p}_{r}^{\perp}=\frac{\hat{s}_{r} \times \hat{n}}{\left|\hat{s}_{r} \times \hat{n}\right|} \\
\hat{p}_{r}^{\|}=\hat{s}_{r} \times \hat{p}_{r}^{\perp}
\end{array}\right.
$$

And then by using Eq. (2.31) and (2.32), the incident, reflected, and transmitted fields can be represented by their TE and TM components:

$$
\vec{E}_{i / r / t}(Q)=E_{i / r / t}^{\perp}(Q) \hat{p}_{i / r / t}^{\perp}+E_{i / r / t}^{\|}(Q) \hat{p}_{i / r / t}^{\|}
$$


where

$$
\left\{\begin{array}{l}
E_{i / r / t}^{\perp}(Q)=\vec{E}_{i / r / t}(Q) \cdot \hat{p}_{i / r / t}^{\perp} \\
E_{i / r / t}^{\|}(Q)=\vec{E}_{i / r / t}(Q) \cdot \hat{p}_{i / r / t}^{\|}
\end{array}\right.
$$

\section{- Fresnel reflection and transmission coefficients}

Fresnel transmission coefficients can be evaluated at $Q$ by imposing boundary conditions on electric and magnetic fields, which is derived in Appendix B.1 and can be expressed as follows:

$$
\left\{\begin{array}{l}
\tau^{\perp}(Q)=\frac{2 \zeta_{2} \cos \theta_{i}}{\zeta_{2} \cos \theta_{i}+\zeta_{1} \cos \theta_{t}} \\
\tau^{\|}(Q)=\frac{2 \zeta_{2} \cos \theta_{i}}{\zeta_{1} \cos \theta_{i}+\zeta_{2} \cos \theta_{t}}
\end{array}\right.
$$

where $\zeta_{1}=\zeta_{0} / \sqrt{\varepsilon_{r_{1}}}$ and $\zeta_{2}=\zeta_{0} / \sqrt{\varepsilon_{r_{2}}}$ are impedances of the medium I and II, respectively, and $\zeta_{0}=120 \pi$ is the impedance of the free space. In the case of illuminating a parabolic reflector or an elliptical mirror, the Fresnel reflection coefficients are calculated by considering a surface made by perfect electric conductor (PEC). Therefore, when the polarizations are defined like Eq. (2.31) and (2.32), the reflection coefficients are constant:

$$
\Gamma^{\perp}=\Gamma^{\|}=-1
$$

\section{- Reflected and transmitted fields}

By using the Fresnel coefficients derived in Eq. (2.35) and (2.36), one can also calculate the reflected field $\vec{E}_{r}$ and the transmitted field $\vec{E}_{t}$. In the case of illuminating a PEC, the reflected field at $Q$ can be obtained by using a compact dyadic expression as follows:

$$
\vec{E}_{r}(Q)=\vec{E}_{i}(Q) \cdot \bar{R}
$$

where $\bar{R}$ is the dyadic reflection coefficient that can be calculated as below:

$$
\bar{R}=\Gamma^{\perp} \hat{p}_{r}^{\perp} \hat{p}_{i}^{\perp}+\Gamma^{\|} \hat{p}_{r}^{\|} \hat{p}_{i}^{\|}
$$

For a transmission problem, the transmitted field at $Q$ can be obtained by using the dyadic transmission coefficient, $\bar{T}$ :

$$
\vec{E}_{t}(Q)=\vec{E}_{i}(Q) \cdot \bar{T}
$$

where $\bar{T}$ is expressed as:

$$
\bar{T}=\tau^{\perp} \hat{p}_{t}^{\perp} \hat{p}_{i}^{\perp}+\tau^{\|} \hat{p}_{t}^{\|} \hat{p}_{i}^{\|}
$$




\subsubsection{Matching layer}

A matching layer is a dielectric layer fabricated on the top of a QO component. Practically, we implement it on a lens to reduce the reflection coefficient. Especially for broadside incidence, we can design the layer in such a way to reduce the reflected power to zero. To study a matching layer, we start with a stratification that consists of a semi-infinite free space region at the top, a dielectric layer with thickness $l_{m}$ below the free space, and a semi-infinite dielectric slab at the bottom, as illustrated in Fig. 2.15a. A plane wave $\vec{E}_{i}$ impinges on the stratification with the incident angle $\theta_{i}$. It is transmitted into the matching layer with the transmitted angle $\theta_{t}^{m}$, and then transmitted again into the dielectric slab with the transmitted angle $\theta_{t}^{d}$.

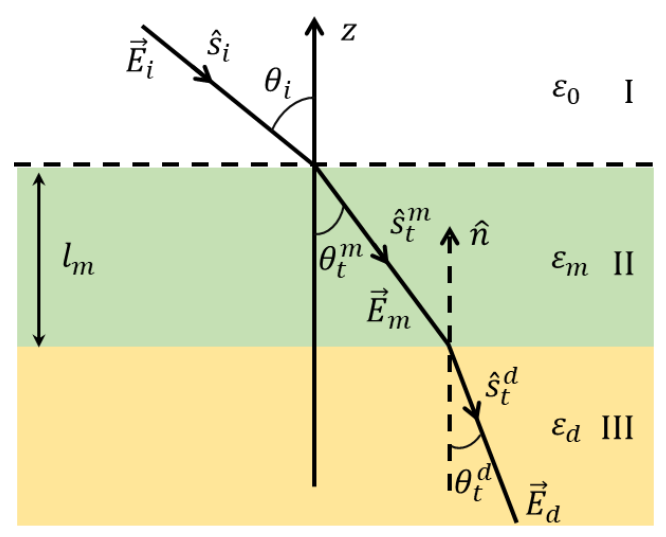

(a)

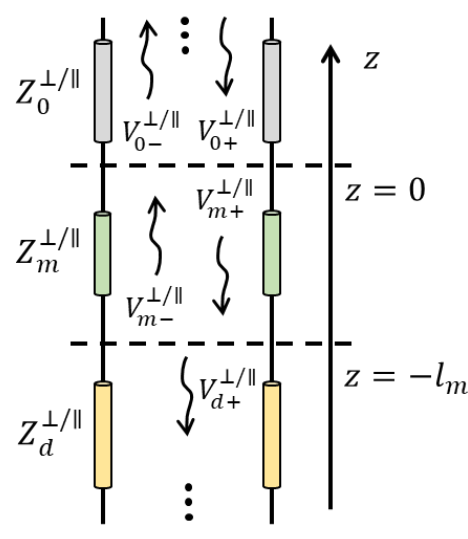

(b)

Figure 2.15: The stratification of a matching layer: (a) The dielectric stratification illuminated by a plane wave with the incident angle $\theta_{i}$. (b) The transversal equivalent transmission line model of the stratification.

The goal of this work is to find the condition for no reflection and derive the transmission coefficients of the stratification, i.e. to calculate $\tau^{\perp / \|}=\left|\vec{E}_{d}^{\perp / \|}\right| /\left|\vec{E}_{i}^{\perp / \|}\right|$. To achieve this, one can represent the stratification by its transversal equivalent transmission line model, as shown in Fig. 2.15b. By solving the transmission line problem (derived in Appendix B.2), one can derive the condition for no reflection: Design a dielectric layer with $\varepsilon_{m}=\sqrt{\varepsilon_{r}}$ and a thickness of $l_{m}=$ $\frac{\lambda_{0}}{4 \sqrt{\varepsilon_{m}}}$. Moreover, the transmission coefficients are derived as:

$$
\left\{\begin{array}{l}
\tau^{\perp}=\frac{V_{d+}^{\perp}\left(z=-l_{m}\right)}{V_{0+}^{\perp}(z=0)} \\
\tau^{\|}=\frac{V_{d+}^{\|}\left(z=-l_{m}\right)}{V_{0+}^{\|}(z=0)} \frac{\cos \theta_{i}}{\cos \theta_{t}^{d}}
\end{array}\right.
$$

where $V_{d+}^{\perp / \|}$ and $V_{m+}^{\perp / \|}$ are progressive voltages that propagate in different layers. 
Fig. 2.16 shows the transmission coefficients for an elliptical silicon $\left(\varepsilon_{r}=11.9\right)$ lens with the maximum truncation angle $\theta_{0} \approx 73^{\circ}$. Here we compare a lens with a matching layer to a lens without. It can be seen in the figure that when a matching layer is added, the transmission coefficients become more symmetric. Moreover, their values are almost 1 (no reflection) around broadside and are still good until $40^{\circ}$. However, one should notice that the critical angle exists for both cases, meaning at around $73^{\circ}$ there is no ray transmitted into the lens.

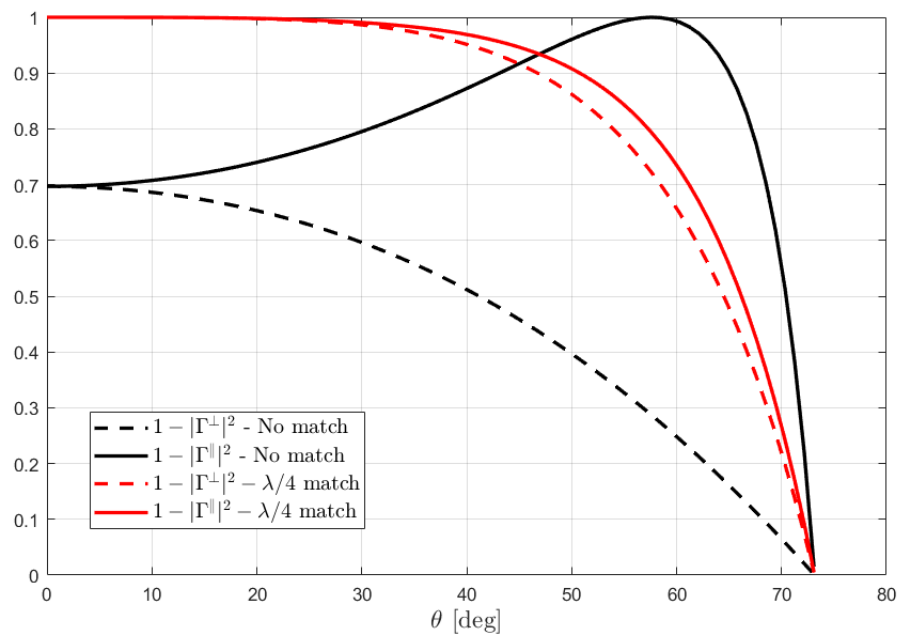

Figure 2.16: Perpendicular $(\perp)$ and parallel $(\|)$ transmission coefficients for an elliptical silicon $\left(\varepsilon_{r}=11.9\right)$ lens with the maximum truncation angle $\theta_{0} \approx 73^{\circ}$. Black lines and red lines represent the cases with and without a matching layer, respectively.

\subsubsection{Visualized ray tracing}

For each QO component, the ray tracing technique can generate the corresponding ray tracing plot which clearly depicts the propagation of incident, reflected and transmitted rays. Fig. 2.17a-f show the ray tracing plots for a parabolic reflector, an elliptical lens, a hemispherical lens, a hyperbolic lens, and an elliptical mirror (source placed at upper or lower focus), respectively. Here we consider the broadside incidence. In each figure, we show incident rays, reflected/transmitted rays, a QO surface, and an observation plane (and dielectric extension). 


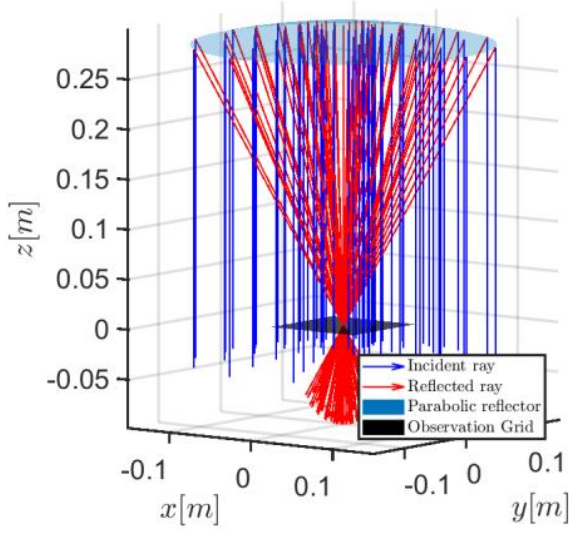

(a)

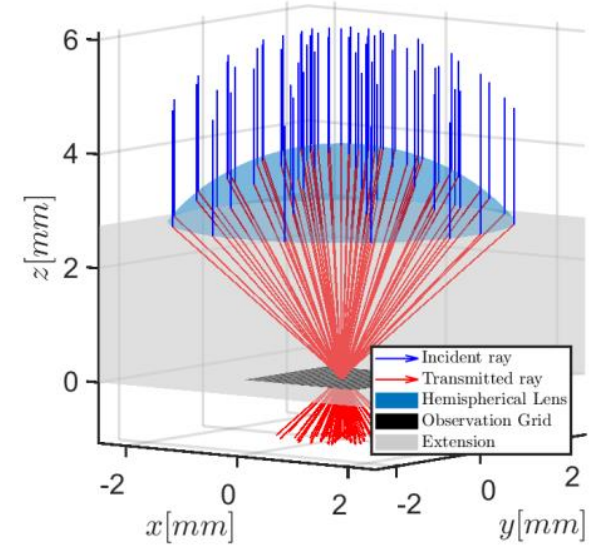

(c)

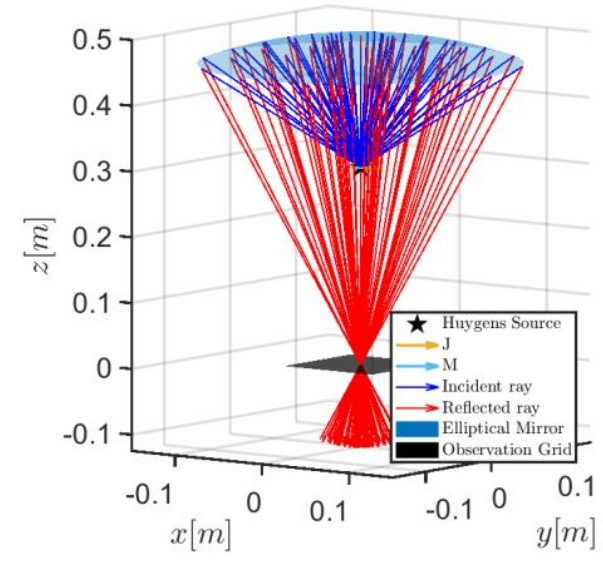

(e)

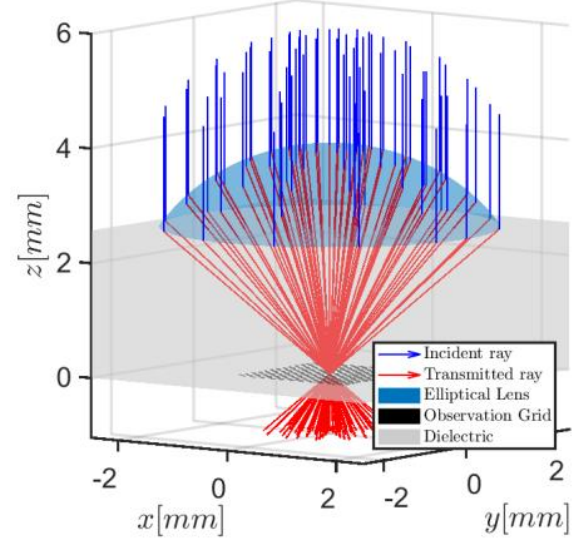

(b)

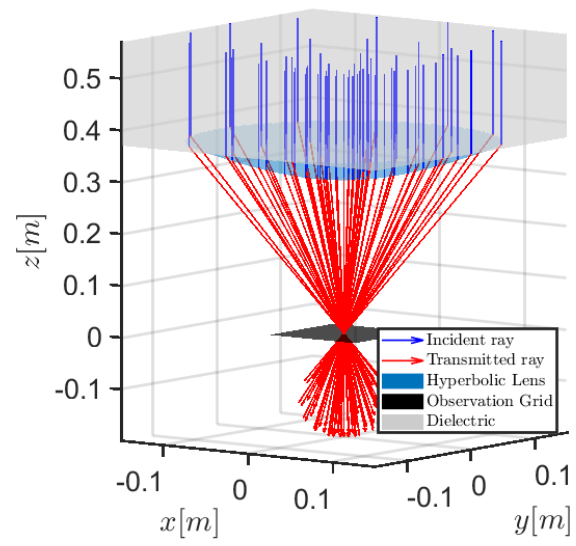

(d)

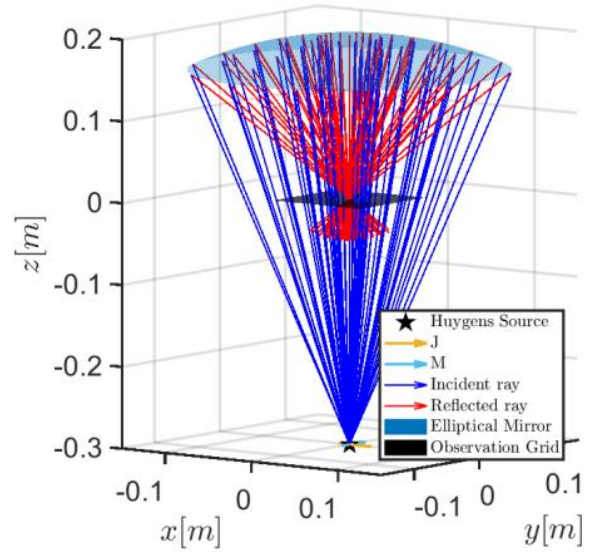

(f)

Figure 2.17: Ray tracing for a (a) Parabolic reflector. (b) Elliptical lens. (c) Hemispherical lens. (d) Hyperbolic lens. (e) Elliptical mirror - source at upper focus. (f) Elliptical mirror - source at lower focus. 


\section{Chapter 3: FO Analysis}

For detector-coupled QO systems, in order to evaluate the performance of the detectors in reception, it has been discussed that one should study the coupling between the QO components and the detectors. Here we consider an imaging system that consists of a QO component, illuminated by a plane wave, and an array of detectors placed at its focal plane. For an absorberbased array, e.g. Kinetic Inductance Detectors (KIDs) based on absorbers, the detectors can be modeled by equivalent Floquet circuits, as described in [37]. The received power is then derived by using the spectral plane wave expansion of the direct fields focalized by the QO component at its focal plane, as described in [15] for broadside incidence, and in [7] for skew incidence. In the case of an antenna-based array, the power captured by the antenna can be evaluated by resorting to the antenna in reception formalism [17, 38], which can be expressed as a field matching between the antenna far field and the spectrum of the incident field. This spectrum is referred to as the plane wave spectrum (PWS).

To calculate the spectrum, one can resort to commercial full-wave simulators, like CST [39] and GRASP software [40]. For an antenna-coupled QO system, one can use the software to calculate the fields over an equivalent sphere centered at the focus of the QO component, i.e. FO sphere. These fields are derived to be proportional to the spectrum. In the case of an absorber-coupled QO system, the software can evaluate the direct fields, and then implements Fourier Transformation (FT) to derive the spectrum. These approaches are rigorous but numerically cumbersome and time-consuming, meaning it is not applicable for large-format array. Alternatively, in the optical domain, a FO method is introduced by E.Wolf in [41]. In our work, we apply the FO formalism to QO systems operating in the THz domain. Within its applicability region, one can use this FO method to calculate the PWS effectively.

In section 3.1, we briefly discuss the PO radiation integral since the FO method is derived from it. Moreover, it can be used as validation on the focal plane fields. The implemented PO analysis based on the PO radiation integral is validated for all QO components. Next in section 3.2, we derive the FO integral, evaluate its applicability region, define FO spheres for all QO components, and derive the spectral representation of focal plane fields. Finally, in section 3.3, we apply the FO method to calculate the PWS. Two types of spectrum are discussed. The first one is a full coherent FO spectrum obtained by calculating a convolution integral. And the second one is a linearized coherent FO spectrum evaluated by applying a linearization approximation. 


\subsection{PO method}

Physical Optics (PO) is a high-frequency technique used to analyze the scattering from arbitrarily shaped and electrically large objects. We use PO in this work due to two reasons. Firstly, PO radiation integral is a starting point to derive the FO method. Secondly, it is a wellestablished technique which provides accurate and reliable results so it is suitable for validating fields on a focal plane. In subsection 3.1.1, we briefly discuss how to calculate the focal plane fields by using the PO radiation integral. And then in subsection 3.1.2, the relevant validation is provided.

\subsubsection{PO radiation integral}

Fig. 3.1a shows the schematic representation of a scattering problem in reflection. Let us assume that an incident wave impinges on an arbitrarily shaped object. The incident field interacts with the object, and the object scatters the field. In order to calculate the scattered field, one can use the love's formulation of the equivalent theorem by replacing the original problem and the object with an equivalent problem that consists of equivalent currents $\vec{J}_{S}$ and $\vec{M}_{S}$ over an equivalent surface surrounding the object as shown in Fig. 3.1b.

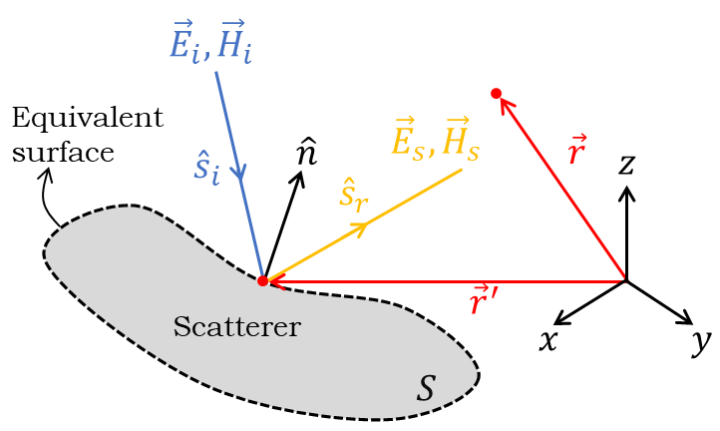

(a)

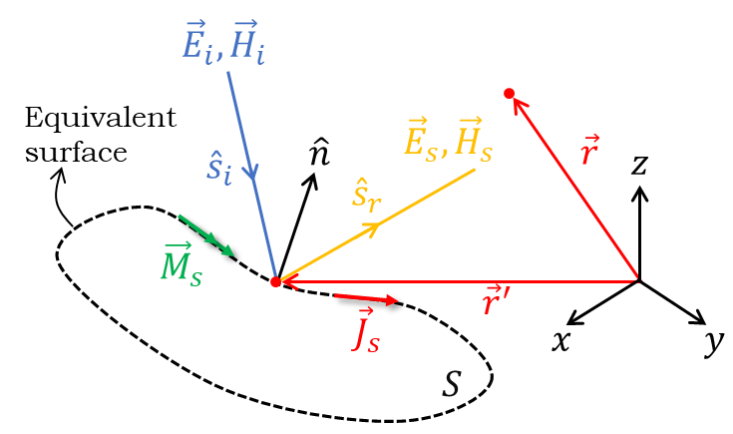

(b)

Figure 3.1: Schematic representation of a scattering problem in reflection analyzed by using the high-frequency approximation: (a) Real geometry. (b) Equivalent problem.

In consequence of the equivalence theorem, the scatterer is removed and these equivalent surface currents radiate in a medium. By using the Green's Functions (GF) in the medium, one can derive the scattered fields in the following form:

$$
\left\{\begin{array}{l}
\vec{E}_{s}(\vec{r})=\iint \tilde{G}_{m}^{e j}\left(\vec{r}-\vec{r}^{\prime}\right) \vec{J}_{s}\left(\vec{r}^{\prime}\right) d \vec{r}^{\prime}+\iint \tilde{G}_{m}^{e m}\left(\vec{r}-\vec{r}^{\prime}\right) \vec{M}_{s}\left(\vec{r}^{\prime}\right) d \vec{r}^{\prime} \\
\vec{H}_{s}(\vec{r})=\iint \tilde{G}_{m}^{h j}\left(\vec{r}-\vec{r}^{\prime}\right) \vec{J}_{s}\left(\vec{r}^{\prime}\right) d \vec{r}^{\prime}+\iint \tilde{G}_{m}^{h m}\left(\vec{r}-\vec{r}^{\prime}\right) \vec{M}_{s}\left(\vec{r}^{\prime}\right) d \vec{r}^{\prime}
\end{array}\right.
$$


where $\vec{E}_{s}$ and $\vec{H}_{s}$ represent the scattered fields; $\tilde{G}_{m}^{e j}, \tilde{G}_{m}^{e m}, \tilde{G}_{m}^{h j}$, and $\tilde{G}_{m}^{h m}$ are GFs in the medium. The equivalent surface currents should satisfy the boundary conditions:

$$
\left\{\begin{array}{l}
\vec{J}_{s}=\hat{n} \times \vec{H}_{t o t} \\
\vec{M}_{s}=-\hat{n} \times \vec{E}_{t o t}
\end{array}\right.
$$

where $\vec{E}_{t o t}$ and $\vec{H}_{t o t}$ represent the total fields on the equivalent surface, and $\hat{n}$ is the normal vector of the surface pointing towards outside from the surface.

In high-frequency scattering scenarios, i.e. the scatterer is large in terms of the wavelength, the PO approximation assumes that the incident field at each point on the object is locally a plane wave impinging on a flat infinite surface, in order to evaluate the surface currents locally at each point instead of solving full-wave integrals or differential equations. Therefore, the scattered field on each point of the equivalent surface can be approximated by using the reflection or transmission dyad; and its direction of propagation is approximated by using the Snell's law. In a reflection problem, the scattered fields, $\left(\vec{E}_{S}, \vec{H}_{s}\right)$, are equal to the reflected fields, $\left(\vec{E}_{r}, \vec{H}_{r}\right)$; and the total fields are the incident fields, $\left(\vec{E}_{i}, \vec{H}_{i}\right)$, plus the reflected fields, i.e. $\vec{E}_{t o t}=\vec{E}_{i}+\vec{E}_{r}$ and $\vec{H}_{t o t}=\vec{H}_{i}+\vec{H}_{r}$. Whereas in a transmission problem, the equivalent surface is chosen in such a way that only the transmitted fields are present outside the surface, meaning the total fields are equal to the transmitted fields: $\vec{E}_{t o t}=\vec{E}_{t}$ and $\vec{H}_{t o t}=\vec{H}_{t}$. Moreover, the GFs can be approximated by their radiating parts; therefore, Eq. (3.1) can be expressed as:

$$
\left\{\begin{array}{l}
\vec{e}_{f}(\vec{r})=\int_{S} j \vec{k} \times \vec{M}_{S} \frac{e^{-j k\left|\vec{r}-\vec{r}^{\prime}\right|}}{4 \pi\left|\vec{r}-\vec{r}^{\prime}\right|} d \vec{r}^{\prime}-j \omega \mu \int_{S}\left[\vec{J}_{s}-\left(\hat{k} \cdot \vec{J}_{S}\right) \hat{k}\right] \frac{e^{-j k\left|\vec{r}-\vec{r}^{\prime}\right|}}{4 \pi\left|\vec{r}-\vec{r}^{\prime}\right|} d \vec{r}^{\prime} \\
\vec{h}_{f}(\vec{r})=-\frac{j k}{\zeta} \int_{S}\left[\vec{M}_{S}-\left(\hat{k} \cdot \vec{M}_{S}\right) \hat{k}\right] \frac{e^{-j k\left|\vec{r}-\vec{r}^{\prime}\right|}}{4 \pi\left|\vec{r}-\vec{r}^{\prime}\right|} d \vec{r}^{\prime}-\int_{S} j \vec{k} \times \vec{J}_{S} \frac{e^{-j k\left|\vec{r}-\vec{r}^{\prime}\right|}}{4 \pi\left|\vec{r}-\vec{r}^{\prime}\right|} d \vec{r}^{\prime}
\end{array}\right.
$$

where $\vec{e}_{f}(\vec{r})$ and $\vec{h}_{f}(\vec{r})$ represent the scattered fields at an observation position $\vec{r}, \vec{r}^{\prime}$ is a point

on the equivalent surface, $\hat{k}=\frac{\vec{r}-\vec{r}^{\prime}}{\left|\vec{r}-\vec{r}^{\prime}\right|}, \vec{k}=k \hat{k}$, and $k$ is the wavenumber in the medium. Here we implement Eq. (3.3) in the tool to calculate the focal plane fields.

\subsubsection{Validation of the implemented PO analysis}

The PO analysis described in Eq. (3.3) is validated in this subsection to demonstrate the reliability of this analysis for later stages. The fields on the focal plane of a QO component are calculated by using the PO analysis and validated by CST [39] or GRASP [40] software. For large QO components, such as parabolic reflectors, elliptical mirrors and hyperbolic lenses, we use GRASP as the reference; while for small lenses like elliptical lenses and hemispherical lenses, we resort to CST. 
In this subsection, we only consider TM-polarized incident fields and show three examples: an elliptical lens with a matching layer, a hemispherical lens without a matching layer, and a hyperbolic lens. Additional examples, including TE validation, are listed in Appendix C. Here we validate both amplitude and phase and show one of the main planes $(y=0)$. Notice that we plot wrapped phase from $-180^{\circ}$ to $180^{\circ}$.

\subsubsection{Elliptical lens}

Here we validate an elliptical lens with a matching layer. Fig. 3.2 shows the $\mathrm{x}$-component of the electric fields on the focal plane of an elliptical silicon lens $\left(\varepsilon_{r}=11.9\right)$ with the diameter of $D_{l}=6 \lambda_{0}\left(f_{0}=300 \mathrm{GHz}\right)$ and the f-number of $f_{\#}^{l}=0.6$. An ideal quarter-wavelength matching layer is added on the top of the lens, i.e. $\varepsilon_{m}=\sqrt{\varepsilon_{r}}=3.45$ and $l_{m}=\lambda_{m} / 4$, where $\lambda_{m}=\lambda_{0} / \sqrt{\varepsilon_{m}}$ is the wavelength in the matching layer. The lens is illuminated by a unitary TM polarized plane wave incoming from the broadside, i.e. $\theta_{s}=0^{\circ}, \phi_{s}=0^{\circ}$. And the focal plane field evaluated by using the PO analysis is compared with the CST simulation. The setup of the simulation in CST is plotted in the inset of the figure. It can be seen in the figure that the amplitude is well validated, and the phase error is acceptable.

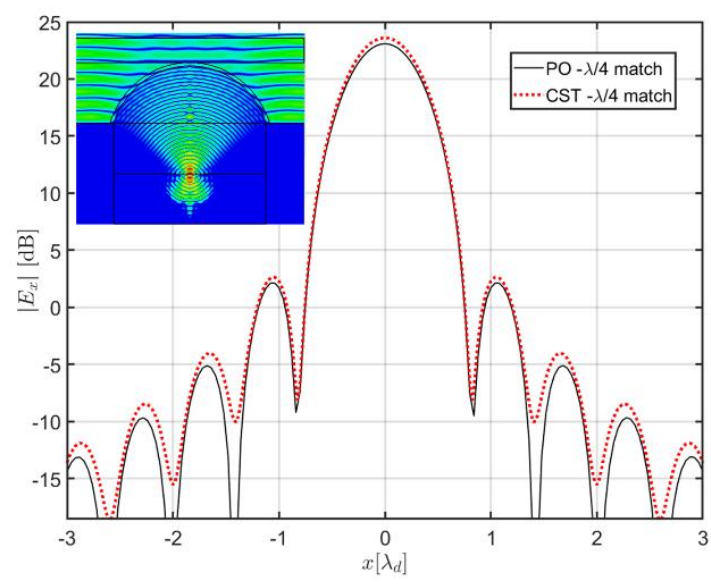

(a)

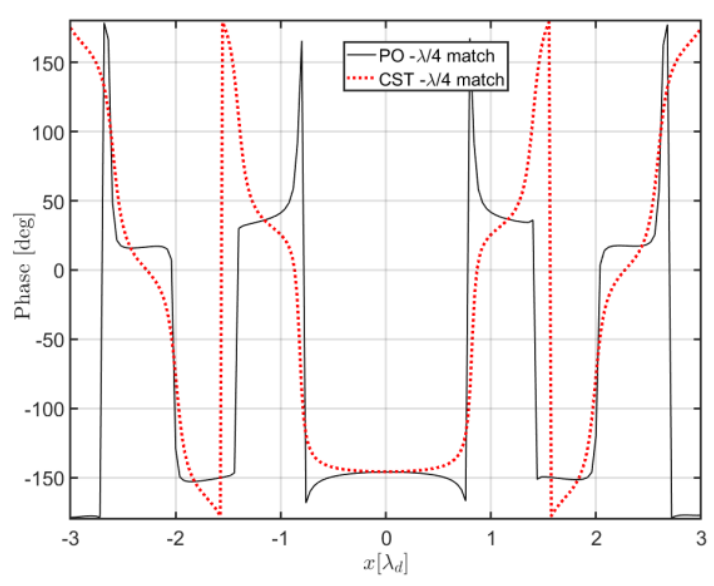

(b)

Figure 3.2: The $\mathrm{x}$-component of the electric fields on the focal plane of an elliptical silicon $\left(\varepsilon_{r}=11.9\right)$ lens with $D_{l}=6 \lambda_{0}\left(f_{0}=300 \mathrm{GHz}\right)$ and $f_{\#}^{l}=0.6$. A matching layer $\left(\varepsilon_{m}=3.45\right)$ with the length $l_{m}=\lambda_{m} / 4$ is added on the top of the lens. The lens is illuminated by a unitary TM polarized plane wave incident from the broadside, i.e. $\theta_{s}=0^{\circ}, \phi_{s}=0^{\circ}$. The focal plane field calculated by using the PO analysis is compared with the CST simulation: (a) Amplitude. (b) Phase. One of the main planes $(y=0)$ is shown. Inset is the configuration in CST. 


\subsubsection{Hemispherical lens}

Fig. 3.3 shows the x-component of the electric fields on the focal plane of a hemispherical silicon $\left(\varepsilon_{r}=11.9\right)$ lens with $D_{h l}=5 \lambda_{0}\left(f_{0}=300 \mathrm{GHz}\right), f_{\#}^{h l}=0.74, R_{s p h}=3 \lambda_{0}$, and $L=$ $0.362 R_{s p h}$. The lens is illuminated by a unitary TM polarized plane wave with the skew angle of $\theta_{s}=20^{\circ}, \phi_{s}=0^{\circ}$. And the focal plane field evaluated by resorting to the PO analysis is compared with the CST simulation. The setup of the simulation in CST is plotted in the inset. As it can be seen, the PO results are in very good agreement with the CST simulation.

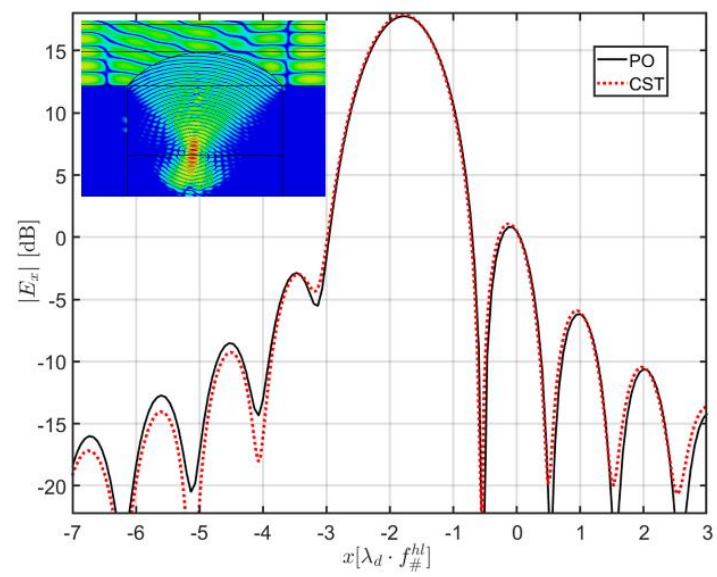

(a)

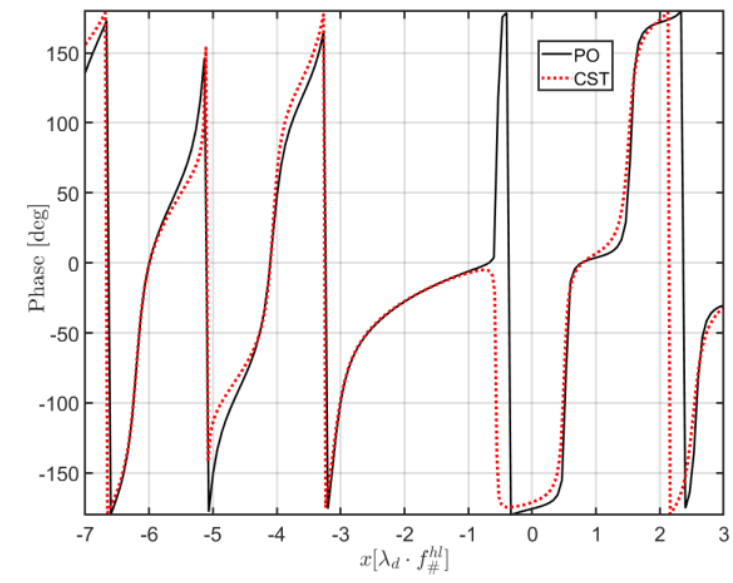

(b)

Figure 3.3: The $\mathrm{x}$-component of the electric fields on the focal plane of a hemispherical silicon $\left(\varepsilon_{r}=11.9\right)$ lens with $D_{h l}=5 \lambda_{0}\left(f_{0}=300 \mathrm{GHz}\right), f_{\#}^{h l}=0.74, R_{s p h}=3 \lambda_{0}$, and $L=0.362 R_{s p h}$, illuminated by a unitary TM polarized plane wave with the skew angle of $\theta_{s}=20^{\circ}, \phi_{s}=0^{\circ}$. The focal plane field calculated by using the PO analysis is compared with the CST simulation: (a) Amplitude. (b) Phase. One of the main planes $(y=0)$ is shown. Inset is the configuration in CST. 


\subsubsection{Hyperbolic lens}

A hyperbolic plastic $\left(\varepsilon_{r}=2\right)$ lens with $D_{h}=100 \lambda_{0}\left(f_{0}=300 \mathrm{GHz}\right)$ and $f_{\#}^{h}=0.6$ is introduced here. It is illuminated by a unitary TM polarized plane wave with the skew angle of $\theta_{s}=3\left(\lambda_{d} / D_{h}\right)=1.22^{\circ}, \phi_{s}=0^{\circ}$, where $\lambda_{d}$ is the wavelength in the dielectric. Fig. 3.4 shows the $\mathrm{x}$-component of the electric fields on the focal plane of the hyperbolic lens. The focal plane field obtained by using the PO analysis is compared with the GRASP simulation. The setup of the simulation in GRASP is plotted in the inset. As it can be seen in the figure, the PO results are in excellent agreement with the GRASP simulation.

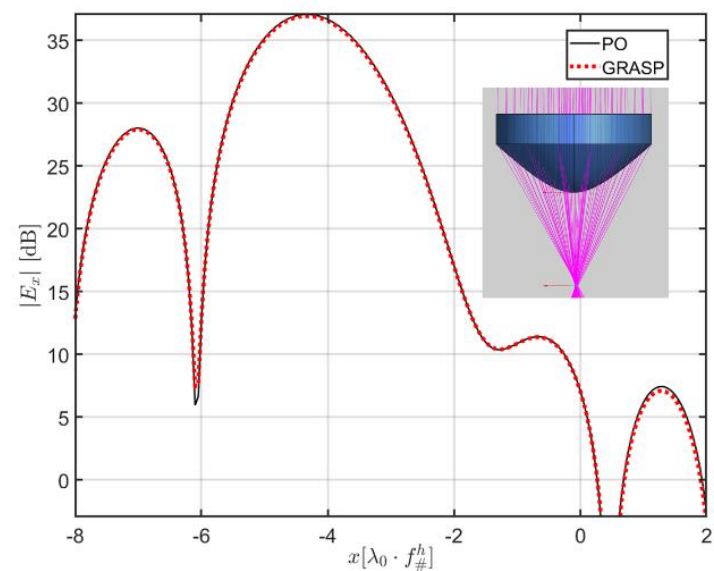

(a)

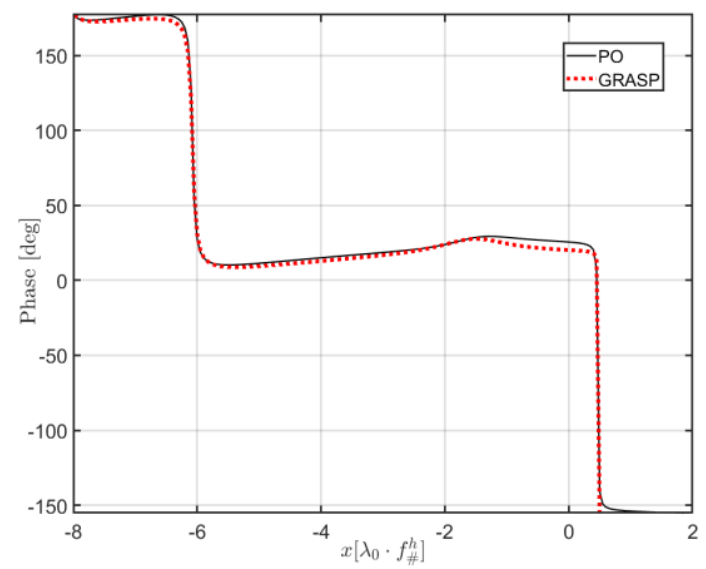

(b)

Figure 3.4: The $x$-component of the electric fields on the focal plane of a hyperbolic plastic $\left(\varepsilon_{r}=2\right)$ lens with $D_{h}=100 \lambda_{0}\left(f_{0}=300 \mathrm{GHz}\right)$ and $f_{\#}^{h}=0.6$, illuminated by a unitary TM polarized plane wave with the skew angle of $\theta_{s}=3\left(\lambda_{d} / D_{h}\right)=1.22^{\circ}, \phi_{s}=0^{\circ}$. The focal plane field calculated by using the PO analysis is compared with the GRASP simulation: (a) Amplitude. (b) Phase. One of the main planes $(y=0)$ is shown. Inset is the configuration in GRASP. 


\subsection{FO integral}

The FO based representation of focal plane fields can be derived by approximating the integrand of the PO radiation integral. One can assume an equivalent sphere centered at the focus of a QO component with the radius $R_{F O}$, referred to as the FO sphere and shown in Fig. 3.5. The electric field, $\vec{E}_{G O}$, and the magnetic field, $\vec{H}_{G O}$, over the FO sphere are called Geometrical Optics (GO) fields in this work. These fields and the procedure to calculate them will be discussed in chapter 4 . The equivalent surface currents $\vec{M}_{G O}$ and $\vec{J}_{G O}$ can be obtained by using the GO fields as:

$$
\left\{\begin{array}{l}
\vec{J}_{G O}=\hat{n} \times \vec{H}_{G O} \\
\vec{M}_{G O}=\vec{E}_{G O} \times \hat{n}
\end{array}\right.
$$

where $\hat{n}$ is the normal vector of the FO sphere defined as $\hat{n}=-\hat{r}^{\prime}$.

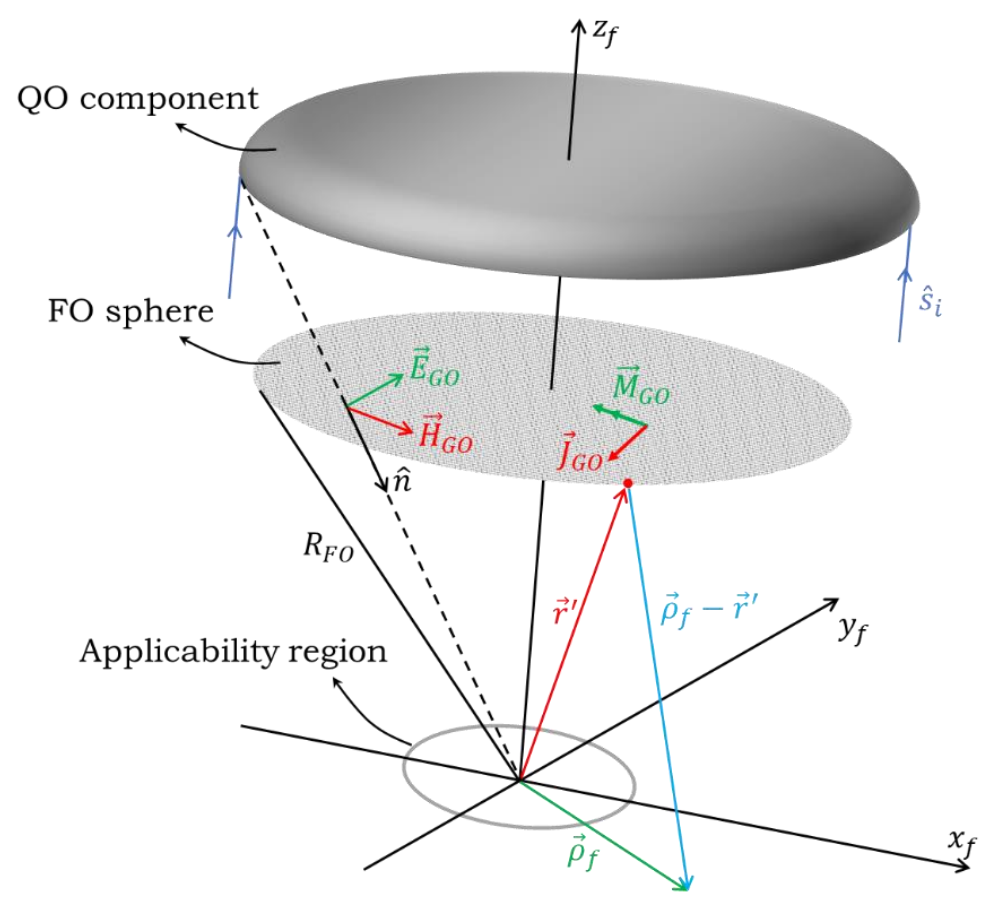

Figure 3.5: Schematic representation of the FO method.

By applying Eq. (3.3) over the FO sphere, the electric field at the focal plane can be calculated as follows:

$$
\begin{aligned}
\vec{e}_{f}\left(\vec{\rho}_{f}\right)=\int_{S_{F O}} j \vec{k} \times \vec{M}_{G O} \frac{e^{-j k\left|\vec{\rho}_{f}-\vec{r}^{\prime}\right|}}{4 \pi\left|\vec{\rho}_{f}-\vec{r}^{\prime}\right|} d \vec{r}^{\prime}- \\
j \omega \mu \int_{S_{F O}}\left[\vec{J}_{G O}-\left(\hat{k} \cdot \vec{J}_{G O}\right) \hat{k}\right] \frac{e^{-j k\left|\vec{\rho}_{f}-\vec{r}^{\prime}\right|}}{4 \pi\left|\vec{\rho}_{f}-\vec{r}^{\prime}\right|} d \vec{r}^{\prime}
\end{aligned}
$$


As it is discussed in [15], Eq. (3.5) can be simplified if we introduce approximations on the unit vector $\hat{k}$ :

$$
\hat{k}=\frac{\vec{\rho}_{f}-\vec{r}^{\prime}}{\left|\vec{\rho}_{f}-\vec{r}^{\prime}\right|} \approx-\hat{r}^{\prime}=\hat{n}
$$

on the amplitude term:

$$
\frac{1}{\left|\vec{\rho}_{f}-\vec{r}^{\prime}\right|} \approx \frac{1}{R_{F O}}
$$

and on the phase term:

$$
\left|\vec{\rho}_{f}-\vec{r}^{\prime}\right| \approx R_{F O}-\vec{\rho}_{f} \cdot \hat{r}^{\prime}+\frac{\rho_{f}^{2}}{2 R_{F O}}
$$

By substituting Eq. (3.6), (3.7) and (3.8) in Eq. (3.5):

$$
\begin{array}{r}
\vec{e}_{f}\left(\vec{\rho}_{f}\right)=\frac{j k e^{-j k R_{F O}} e^{-j k \frac{\rho_{f}^{2}}{2 R_{F O}}}}{4 \pi R_{F O}} \int_{S_{F O}}\left[\vec{E}_{G O}-\left(\hat{n} \cdot \vec{E}_{G O}\right) \hat{n}\right] e^{j k \vec{\rho}_{f} \cdot \hat{r}^{\prime}} d \vec{r}^{\prime}- \\
\frac{j k \zeta e^{-j k R_{F O}} e^{-j k \frac{\rho_{f}^{2}}{2 R_{F O}}}}{4 \pi R_{F O}} \int_{S_{F O}}\left(\hat{n} \times \vec{H}_{G O}\right) e^{j k \vec{\rho}_{f} \cdot \hat{r}^{\prime}} d \vec{r}^{\prime}
\end{array}
$$

Moreover, one can assume the GO magnetic field is orthogonal to the electric field with respect to the normal to the FO sphere, $\hat{n}$, as follows:

$$
\vec{H}_{G O}=\frac{1}{\zeta} \hat{n} \times \vec{E}_{G O}
$$

For the FO sphere, $d \vec{r}^{\prime}$ can be expressed as $d \vec{r}^{\prime}=R_{F O}^{2} \sin \theta d \theta d \phi$. By substituting $d \vec{r}^{\prime}$ and $\vec{H}_{G O}$ in Eq. (3.9), one can represent the electric focal plane field by the electric GO field only, as:

$$
\vec{e}_{f}\left(\vec{\rho}_{f}\right)=\frac{j k e^{-j k R_{F O}} e^{-j k \frac{\rho_{f}^{2}}{2 R_{F O}}}}{4 \pi R_{F O}} \int_{S_{F O}} 2 \vec{E}_{G O}(\theta, \phi) e^{j k \vec{\rho}_{f} \cdot \hat{r}^{\prime}} R_{F O}^{2} \sin \theta d \theta d \phi
$$

Eq. (3.11) is referred to as the FO integral, for the electric field on the focal plane. And then we will discuss its applicability region. 


\subsubsection{FO applicability region}

The vector, amplitude and phase approximations described in Eq. (3.6), (3.7), and (3.8) lead to an applicability region for the FO integral. This region is derived by imposing constraints on the error for each approximation: By choosing the maximum tolerable error for the vector approximation as $20 \%$, the one for the amplitude as $20 \%$, and the one for the phase as $\pi / 8$. The diameter of the FO applicability region is then derived as [15]:

$$
\operatorname{Diam}_{F O}=f_{\#} \min \left(0.4 D, \sqrt{2 f_{\#} D \lambda}\right)
$$

where $D$ is the diameter of a QO component and $f_{\#}=R_{F O} / D$ is defined as its f-number. From this expression, it is apparent that for a fixed diameter $D$, the FO applicability region is proportional to the radius of a FO sphere, $R_{F O}$. Therefore, to maximize this region, $R_{F O}$ should be selected as large as possible. It is worth mentioning that this region is valid when the $\mathrm{f}$ number $f_{\#}$ is relatively small; however, when $f_{\#}$ is large, e.g. $f_{\#}=4$, Eq. (3.12) is not accurate enough. In such a case, the condition for the phase approximation should be stricter. If we expand the term $\left|\vec{\rho}_{f}-\vec{r}^{\prime}\right|$ to its second order, the phase approximation described in Eq. (3.8) becomes:

$$
\left|\vec{\rho}_{f}-\vec{r}^{\prime}\right| \approx R_{F O}-\vec{\rho}_{f} \cdot \hat{r}^{\prime}+\frac{\rho_{f}^{2}}{2 R_{F O}}\left[1-\left(\hat{\rho}_{f} \cdot \hat{r}^{\prime}\right)^{2}\right]+\frac{\rho_{f}^{3}}{2 R_{F O}^{2}}\left(\hat{\rho}_{f} \cdot \hat{r}^{\prime}\right)
$$

By neglecting the second- and the third- order terms in Eq. (3.13) with a phase error $\pi / 8$, one can find a new region for the phase approximation:

$$
\frac{4 \rho_{f}^{3} D}{R_{F O}^{3}}+\frac{2 \rho_{f}^{2} D}{R_{F O}^{3}}-\lambda \leq 0
$$

where $\rho_{f}=\left|\vec{\rho}_{f}\right|$. If the positive and real solution of the cubic inequality Eq. (3.14) is defined as $\rho_{p h}^{F O}$, the FO applicability region is re-evaluated as follows:

$$
\operatorname{Diam}_{F O, \text { mod }}=\min \left(0.4 f_{\#} D, 2 \rho_{p h}^{F O}\right)
$$

Explicit derivation of this modified region is discussed in Appendix D.

\subsubsection{Parameterizing FO spheres for QO components}

Fig. 3.6 shows a FO sphere $S_{F O}$ centered at the focus $O$ of a QO surface $S$. Similar to parameterize a QO surface, a point $Q_{F O}$ on the FO sphere can be parameterized as follows in $(\theta, \phi)$ variable:

$$
\left\{\begin{array}{l}
Q_{F O, x}=R_{F O} \sin \theta \cos \phi \\
Q_{F O, y}=R_{F O} \sin \theta \sin \phi \\
Q_{F O, z}=R_{F O} \cos \theta
\end{array}\right.
$$


And in $(u, v)$ variable:

$$
\left\{\begin{array}{l}
Q_{F O, x}=R_{F O} u \\
Q_{F O, y}=R_{F O} v \\
Q_{F O, z}=R_{F O} \sqrt{1-\left(u^{2}+v^{2}\right)}
\end{array}\right.
$$

The normal vector of the FO sphere, $\hat{n}_{F O}$, can be calculated analytically as:

$$
\hat{n}_{F O}=\sin \theta \cos \phi \hat{x}+\sin \theta \sin \phi \hat{y}+\cos \theta \hat{z}
$$

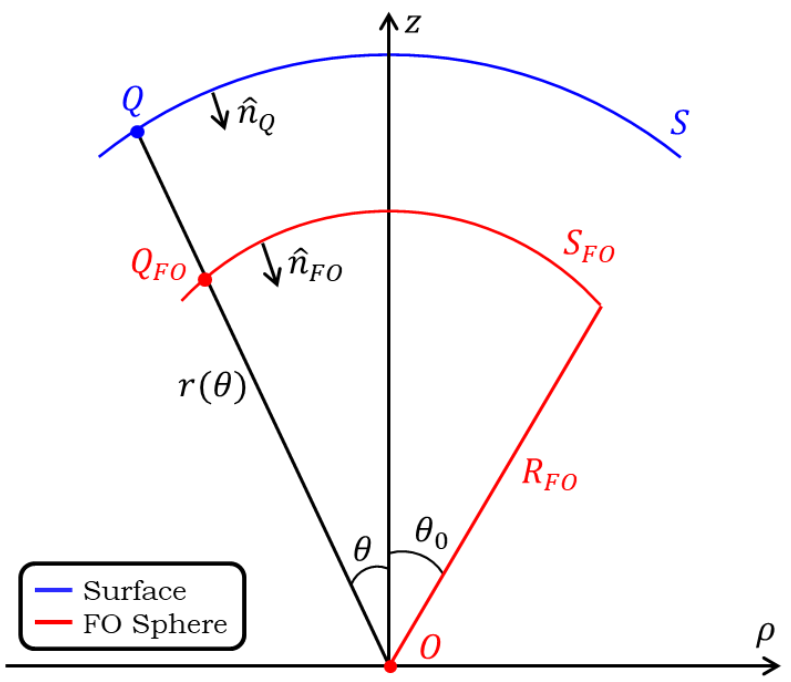

Figure 3.6: Sketch of a generic QO surface $S$ and its FO sphere $S_{F O}$.

The radius of the FO sphere, $R_{F O}$, can be selected arbitrarily. However, in order to maximize the FO applicability region, $R_{F O}$ should be chosen as large as possible, as described in Eq. (3.12). For different QO components, $R_{F O}$ is selected as follows. In the case of a parabolic reflector and a hyperbolic lens, as shown in Fig. 3.7 and 3.8, respectively, the radius is equal to the focal distance:

$$
R_{F O}=f
$$

For an elliptical lens and a hemispherical lens, as depicted in Fig. 3.9 and 3.10, respectively, the radius is equal to the rim distance:

$$
R_{F O}=R_{l}
$$

For an elliptical mirror (Fig. 3.11), the observation plane can be placed at either the lower focal plane or the upper focal plane. When the observation plane is the lower focal plane (Fig. 3.11a), the radius is the same as the rim distance:

$$
R_{F O}=R_{2 l}
$$

When the observation plane is the upper focal plane (Fig. 3.11b), the radius is expressed as:

$$
R_{F O}=a-c
$$


where $a$ is the semi-major axis and $c$ is the focal distance.

It is worth noting that here the f-number is generally defined as $f_{\#}=R_{F O} / D$ for all QO components, which agrees with the previous definition introduced in section 2.1.3.

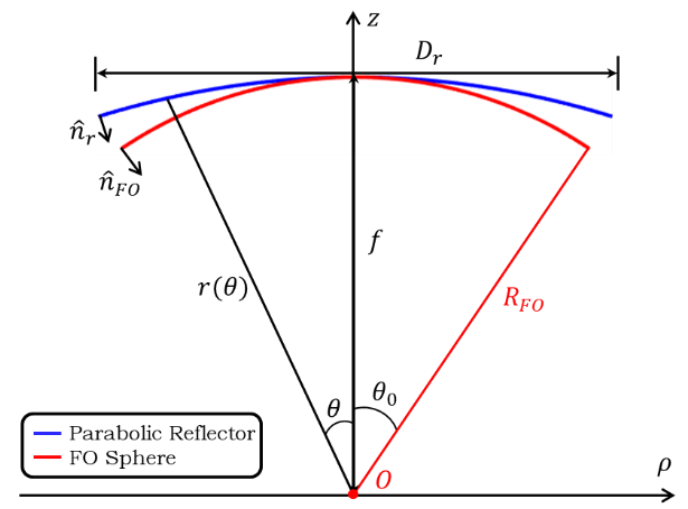

Figure 3.7: 2D geometry of a parabolic reflector.

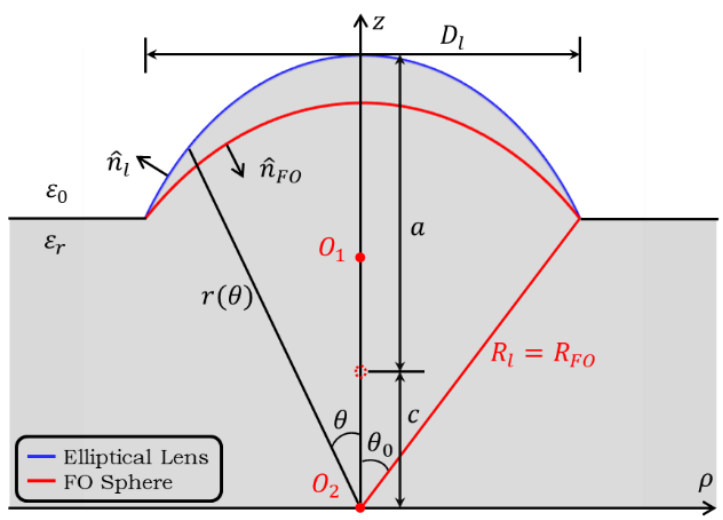

Figure 3.9: 2D geometry of an elliptical lens.

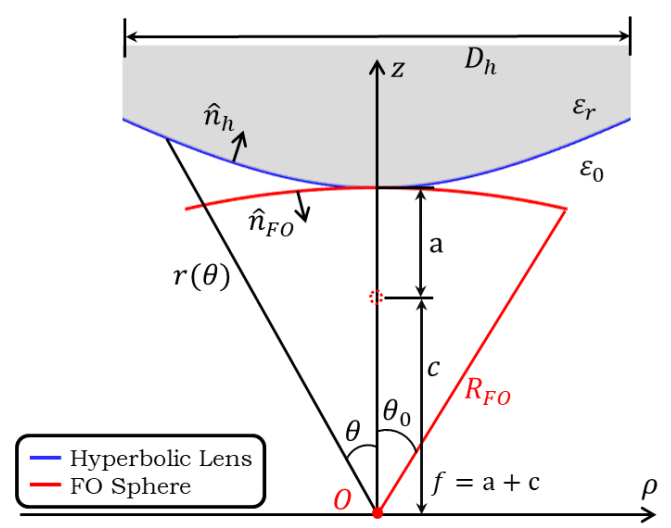

Figure 3.8: 2D geometry of a hyperbolic lens

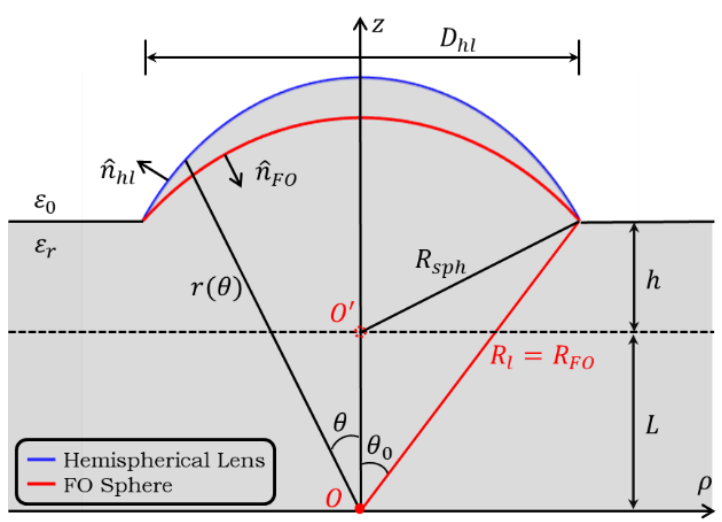

Figure 3.10: 2D geometry of a hemispherical lens.

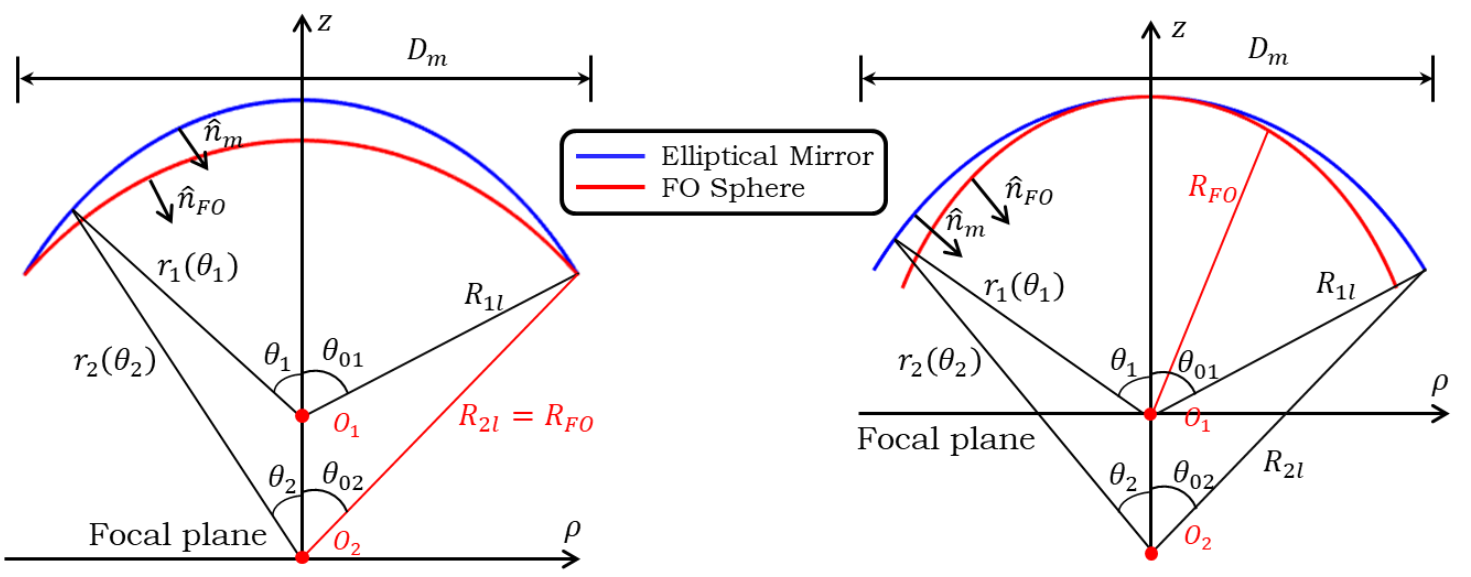

(a)

(b)

Figure 3.11: 2D geometry of an elliptical mirror: (a) Observation at lower focal plane. (b) Observation at upper focal plane. 


\subsubsection{Spectral representation}

In this subsection, the focal plane field calculated by using the FO integral, Eq. (3.11), is expressed by three types of spectral representation, namely spherical, cylindrical and Cartesian representation.

\section{- Spherical representation}

In Eq. (3.11), the focal plane field is expressed in spherical coordinate system. By considering $\vec{\rho}_{f}=x_{f} \hat{x}+y_{f} \hat{y}$, and $\hat{r}^{\prime}=\sin \theta \cos \phi \hat{x}+\sin \theta \sin \phi \hat{y}+\cos \theta \hat{z}$, Eq. (3.11) becomes:

$$
\vec{e}_{f}\left(\vec{\rho}_{f}\right)=\frac{j k R_{F O} e^{-j k R_{F O}} e^{-j k \frac{\rho_{f}^{2}}{2 R_{F O}}}}{2 \pi} \int_{0}^{2 \pi} \int_{0}^{\theta_{0}} \vec{E}_{G O} e^{j k_{x} x_{f}} e^{j k_{y} y_{f}} \sin \theta d \theta d \phi
$$

where $k_{x}=k \sin \theta \cos \phi$ and $k_{y}=k \sin \theta \sin \phi$ are spectral parameters.

\section{- Cylindrical representation}

Moreover, one can express Eq. (3.23) in a cylindrical representation by substituting the Jacobian: $\frac{\partial(\theta, \phi)}{\partial\left(k_{\rho}, \phi\right)}=\frac{1}{\sqrt{k^{2}-k_{\rho}^{2}}}$, where $k_{\rho}=k \sin \theta$ :

$$
\vec{e}_{f}\left(\vec{\rho}_{f}\right)=\frac{j R_{F O} e^{-j k R_{F O}} e^{-j k \frac{\rho_{f}^{2}}{2 R_{F O}}}}{2 \pi} \int_{0}^{2 \pi} \int_{0}^{\theta_{0}} \vec{E}_{G O} e^{j k_{x} x_{f}} e^{j k_{y} y_{f}} \frac{k_{\rho}}{k_{z}} d k_{\rho} d \phi
$$

where $k_{z}=\sqrt{k^{2}-k_{\rho}^{2}}$.

\section{- Cartesian representation}

In some cases, the Cartesian representation could be more convenient, which can be derived by applying the Jacobian: $\frac{\partial\left(k_{\rho}, \phi\right)}{\partial\left(k_{x}, k_{y}\right)}=\frac{1}{k_{\rho}}$ :

$$
\vec{e}_{f}\left(\vec{\rho}_{f}\right)=\frac{j R_{F O} e^{-j k R_{F O}} e^{-j k \frac{\rho_{f}^{2}}{2 R_{F O}}}}{2 \pi} \int_{0}^{2 \pi} \int_{0}^{\theta_{0}} \vec{E}_{G O} e^{j k_{x} x_{f}} e^{j k_{y} y_{f}} \frac{1}{k_{z}} d k_{x} d k_{y}
$$

If we combine some terms in Eq. (3.25) together and call it $\vec{E}_{F O}\left(k_{x}, k_{y}\right)$ :

$$
\vec{E}_{F O}\left(k_{x}, k_{y}\right)=\frac{j 2 \pi R_{F O} e^{-j k R_{F O}}}{k_{z}} \vec{E}_{G O}\left(k_{x}, k_{y}\right) \operatorname{circ}\left(k_{\rho}, k_{\rho 0}\right)
$$


the Cartesian representation can be expressed as:

$$
\vec{e}_{f}\left(\vec{\rho}_{f}\right)=\frac{1}{4 \pi^{2}} e^{-j k \frac{\rho_{f}^{2}}{2 R_{F O}}} \int_{-\infty}^{\infty} \int_{-\infty}^{\infty} \vec{E}_{F O}\left(k_{x}, k_{y}\right) e^{j k_{x} x_{f}} e^{j k_{y} y_{f}} d k_{x} d k_{y}
$$

In Eq. (3.26), $\operatorname{circ}\left(k_{\rho}, k_{\rho 0}\right)$ is a circular region that constrains the spectrum within $k_{\rho} \in$ $\left(0, k_{\rho 0}\right)$, with $k_{\rho 0}=k \sin \theta_{0}$, and $\vec{E}_{G O}\left(k_{x}, k_{y}\right)$ is the GO field on the FO sphere, which will be explicitly discussed in chapter 4 . It should be noticed that $\vec{E}_{F O}\left(k_{x}, k_{y}\right)$ represents the spectrum of $\vec{e}_{f}\left(\vec{\rho}_{f}\right)$, i.e. the PWS, if the quadratic phase term $e^{-j k \rho_{f}^{2} / 2 R_{F O}}$ is neglected. Therefore, a simple consideration is that if the observation point $\vec{\rho}_{f}$ is not far away from the focus, one can ignore the quadratic phase term and assume $\vec{E}_{F O}\left(k_{x}, k_{y}\right)$ to be the PWS.

However, this region could be very small compared with the FO validity region. This means, in practice, if we consider a large focal plane array (FPA), we cannot accurately estimate the PWS for the detectors that are far away from the center. In fact, for an FPA, we only need an accurate PWS over the feed antenna aperture, meaning we can evaluate the quadratic phase term at the center of the aperture. This approximation could improve the applicability region for PWS in some extent, but still not useful for a feed that are far away from the center. Consequently, in most cases, the quadratic phase term cannot be neglected and $\vec{E}_{F O}\left(k_{x}, k_{y}\right)$ is not the complete spectral representation of the focal plane field. To solve this issue, in the following section, we propose a coherent FO method to evaluate an accurate PWS within the entire FO applicability region.

\subsection{Coherent FO}

In this section, we will discuss a FO method called Coherent FO (CFO) to evaluate the PWS of the focal plane field. It is called CFO since the quadratic phase term is included in the spectrum evaluations. Two types of spectrum can be derived by using CFO: The first one is a full coherent FO (CFO) spectrum evaluated by calculating a convolution integral; The second one is a linearized CFO spectrum, evaluated locally within an applicability region that indicates the maximum feed aperture diameter of an antenna. 


\subsubsection{Full CFO spectrum}

It has been discussed in section 3.2.3 that the quadratic phase term in the FO integral cannot be neglected. If we represent it as a function:

$$
f\left(\vec{\rho}_{f}\right)=e^{-j k \frac{\left|\vec{\rho}_{f}\right|^{2}}{2 R_{F O}}}
$$

and represent the remaining terms in Eq. (3.27) as another function:

$$
\vec{e}_{F O}\left(\vec{\rho}_{f}\right)=\frac{1}{4 \pi^{2}} \int_{-\infty}^{\infty} \int_{-\infty}^{\infty} \vec{E}_{F O}\left(k_{x}, k_{y}\right) e^{j k_{x} x_{f}} e^{j k_{y} y_{f}} d k_{x} d k_{y}
$$

we can express the focal plane field as follows:

$$
\vec{e}_{f}\left(\vec{\rho}_{f}\right)=f\left(\vec{\rho}_{f}\right) \vec{e}_{F O}\left(\vec{\rho}_{f}\right)
$$

By performing FT on Eq. (3.30) and using FT properties, one can obtain a convolution integral:

$$
\begin{aligned}
\vec{E}_{f}\left(k_{x}, k_{y}\right)=F\left(k_{x}, k_{y}\right) * \vec{E}_{F O}\left(-k_{x},-k_{y}\right) \\
=\iint_{-\infty}^{\infty} \vec{E}_{F O}\left(-k_{x}^{\prime},-k_{y}^{\prime}\right) F\left(k_{x}-k_{x}^{\prime}, k_{y}-k_{y}^{\prime}\right) d k_{x}^{\prime} d k_{y}^{\prime}
\end{aligned}
$$

where $F\left(k_{x}, k_{y}\right)=F T\left\{f\left(\vec{\rho}_{f}\right)\right\}$ can be expressed analytically:

$$
F\left(k_{x}, k_{y}\right)=\left(\frac{1}{2 \pi}\right)^{2}\left(\frac{2 \pi R_{F O}}{k}\right) e^{-j \frac{\pi}{2}} e^{j \frac{R_{F O}}{2 k}\left(k_{x}^{2}+k_{y}^{2}\right)}
$$

By using Eq. (3.31), one can represent the field on the focal plane, $\vec{e}_{f}\left(\vec{\rho}_{f}\right)$, as a plane wave expansion:

$$
\vec{e}_{f}\left(\vec{\rho}_{f}\right)=\frac{1}{4 \pi^{2}} \iint_{-\infty}^{\infty} \vec{E}_{f}\left(-k_{x},-k_{y}\right) e^{j k_{x} x_{f}} e^{j k_{y} y_{f}} d k_{x} d k_{y}
$$

and here the term $\vec{E}_{f}\left(-k_{x},-k_{y}\right)$ is the PWS of the focal plane field:

$$
\vec{E}_{f}\left(-k_{x},-k_{y}\right)=F\left(-k_{x},-k_{y}\right) * \vec{E}_{F O}\left(k_{x}, k_{y}\right)
$$

It is worth noting that since we do not use any approximation, the applicability region for this coherent FO spectrum is the same as the FO validity region. Moreover, since the spectrum in Eq. (3.34) is calculated by using a convolution integral, it is not bounded from 0 to $k_{\rho 0}$. In practice, it should be integrated from 0 to a large $k_{\rho}$ value, in order to reach a convergent condition in the numerical calculation, which is numerically cumbersome and time-consuming. Accordingly, we use a linearization approximation on the quadratic phase term to simplify the calculation of convolution. 


\subsubsection{Linearized CFO spectrum}

The derivation of the full CFO spectrum is rigorous; however, it is numerically cumbersome, which makes it impractical. Alternatively, we can consider a local representation of PWS since we only need accurate spectrum over a specific feed aperture. Here we introduce a linearization on the quadratic phase term to simplify the convolution integral. To start with, as is depicted in Fig. 3.12, one can represent an observation point $\vec{\rho}_{f}$ at the surrounding of a local system located at $O^{\prime}$, i.e. $\vec{\rho}_{f}=\vec{\rho}_{o}+\vec{\rho}^{\prime}$, where $\vec{\rho}^{\prime}$ is a position on the focal plane in the neighborhood of $\vec{\rho}_{o}$. In the case of an FPA, the dashed circle represents the feed aperture of an antenna located at $O^{\prime}$.

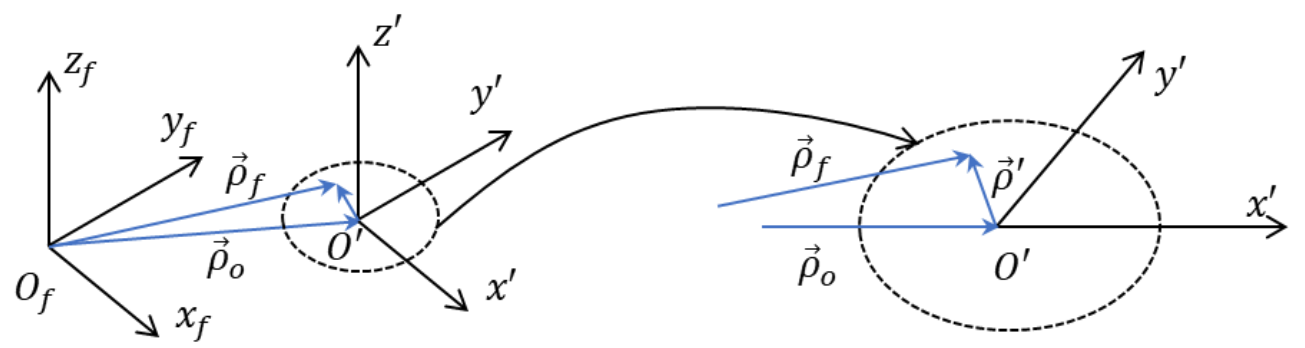

Figure 3.12: Observation point $\vec{\rho}_{f}$ represented by a local system located at $\vec{\rho}_{o}$. The dashed circle represents the surrounding of $\vec{\rho}_{o}$.

By substituting $\vec{\rho}_{f}$ in Eq. (3.28), the quadratic phase term can be represented in the surrounding of $\vec{\rho}_{o}$ :

$$
f\left(\vec{\rho}_{o}+\vec{\rho}^{\prime}\right)=e^{-j k \frac{\left|\vec{\rho}_{O}\right|^{2}}{2 R_{F O}}} e^{-j k \frac{\vec{\rho}_{o} \cdot \vec{\rho}^{\prime}}{R_{F O}}} e^{-j k \frac{\left|\vec{\rho}^{\prime}\right|^{2}}{2 R_{F O}}}
$$

Eq. (3.35) can be approximated to a linear function, by neglecting the phase term $e^{-j k \frac{\left.||^{\prime}\right|^{2}}{2 R_{F O}}}$ :

$$
f\left(\vec{\rho}_{o}+\vec{\rho}^{\prime}\right) \approx e^{-j k \frac{\left|\vec{\rho}_{O}\right|^{2}}{2 R_{F O}}} e^{-j k \frac{\vec{\rho}_{o} \cdot \vec{\rho}^{\prime}}{R_{F O}}}
$$

If we set the tolerable error for this phase linearization approximation as $\pi / 8$, the applicability region of Eq. (3.36) surrounding $\vec{\rho}_{o}$ can be found as:

$$
k \frac{\left|\vec{\rho}^{\prime}\right|^{2}}{2 R_{F O}} \leq \frac{\pi}{8} \Rightarrow\left|\vec{\rho}^{\prime}\right| \leq \sqrt{\frac{f_{\#} D \lambda}{8}}
$$

It is worth noting that in the case of an FPA, Eq. (3.37) also indicates the maximum diameter of a feed aperture which can be analyzed using the linearization approximation: 


$$
D_{f} \leq \sqrt{\frac{f_{\#} D \lambda}{2}}
$$

By neglecting the phase term, the FT of $f\left(\vec{\rho}_{o}+\vec{\rho}^{\prime}\right)$ can be calculated as:

$$
F\left(k_{x}, k_{y}\right)=e^{-j k \frac{\left|\vec{\rho}_{o}\right|^{2}}{2 R_{F O}}} \delta\left(\vec{k}_{\rho}-\vec{k}_{o}\right)
$$

where $\vec{k}_{\rho}=k_{x} \hat{x}+k_{y} \hat{y}$ and $\vec{k}_{o}=\frac{k}{R_{F O}} \vec{\rho}_{o}$. When we substitute $\vec{\rho}_{f}$ in Eq. (3.29), we can represent $\vec{e}_{F O}$ by using $\vec{\rho}^{\prime}$ :

$$
\vec{e}_{F O}\left(\vec{\rho}^{\prime}\right)=\frac{1}{4 \pi^{2}} \iint_{-\infty}^{\infty} \vec{E}_{F O}\left(-k_{x},-k_{y}\right) e^{-j k_{x}\left(x^{\prime}+x_{o}\right)} e^{-j k_{y}\left(y^{\prime}+y_{o}\right)} d k_{x} d k_{y}
$$

and the FT of $\vec{e}_{F O}\left(\vec{\rho}^{\prime}\right)$ is calculated as follows:

$$
F T\left\{\vec{e}_{F O}\left(\vec{\rho}^{\prime}\right)\right\}=\vec{E}_{F O}\left(-k_{x},-k_{y}\right) e^{-j k_{x} x_{o}} e^{-j k_{y} y_{o}}
$$

Therefore, by using Eq. (3.39) and (3.41), one can obtain the FT of the focal plane field with a local representation:

$$
\begin{aligned}
\vec{E}_{f}\left(k_{x}, k_{y}\right)=F\left(k_{x}, k_{y}\right) *\left[\vec{E}_{F O}\left(-k_{x},-k_{y}\right) e^{-j k_{x} x_{o}} e^{-j k_{y} y_{o}}\right] \\
=e^{-j k \frac{\left|\vec{\rho}_{o}\right|^{2}}{2 R_{F O}} \vec{E}_{F O}\left(-\vec{k}_{\rho}+\vec{k}_{o}\right) e^{-j\left(k_{x}-k_{x o}\right) x_{o}} e^{-j\left(k_{y}-k_{y o}\right) y_{o}}}
\end{aligned}
$$

In Eq. (3.42), it is found that $\vec{E}_{f}\left(k_{x}, k_{y}\right)$ is a linear translation of $\vec{E}_{F O}$ in the spectral domain. Moreover, the focal plane field can be represented surrounding $\vec{\rho}_{o}$ as a plane wave expansion:

$$
\vec{e}_{f}\left(\vec{\rho}^{\prime}\right)=\frac{1}{4 \pi^{2}} \iint_{-\infty}^{\infty} \vec{E}_{f}\left(-k_{x},-k_{y}\right) e^{j k_{x} x^{\prime}} e^{j k_{y} y^{\prime}} d k_{x} d k_{y}
$$

where $\vec{E}_{f}\left(-k_{x},-k_{y}\right)$ is the coherent FO spectrum of the focal plane field, evaluated by using the linearization approximation on the quadratic phase term:

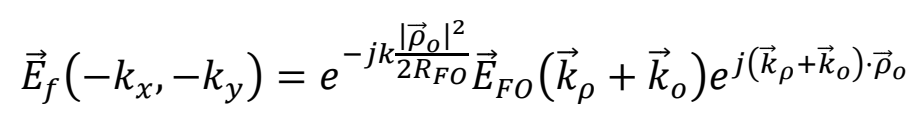

Compared with the full CFO spectrum, this linearized spectrum is less cumbersome and does not exhibit a convergent issue mentioned in the convolution method, since it calculates the PWS locally for a feed aperture by performing a translation of the spectrum in the spectral domain. The applicability region for this CFO is the same as the FO validity region. However, the diameter of the feed aperture is limited by $D_{f}$ which is defined in Eq. (3.38). In this work, we only apply the coherent FO with the linearization approximation, and validation of this CFO method will be discussed in chapter 5 . 


\section{Chapter 4: GO fields on a FO sphere}

In chapter 3, we have discussed that deriving a PWS representation of the focal plane field is significant when evaluating the performance of absorber- and antenna- coupled QO systems. Moreover, we have analyzed that PWS is proportional to the field scattered by a QO component on its FO sphere, which for electrically large scatterers can be approximated with the GO field. Therefore, in this chapter, we will extensively discuss the derivation of the GO fields for each QO component.

In section 4.1, we discuss an asymptotic procedure to evaluate GO fields. It is applied here for general reflection and transmission problems. Next, in section 4.2, we derive analytical closed-form expressions for GO fields, first for a case with broadside incidence; and then for one with slightly off-broadside incidence.

\subsection{GO ray fields}

In this work, the fields scattered by a QO component and propagating to its FO sphere is calculated. In other words, one should describe the wave propagation in a scattering problem. To do so, one can resort to the Geometrical Optics (GO) technique. GO is commonly used in high-frequency scattering scenarios (i.e. when the scatterer is large in terms of the wavelength) to determine wave propagation for both incident and scattered fields, including amplitude, phase, and polarization [13]. In this method, EM waves can be approximated as tubes of rays propagating in a homogenous medium from one point to another. Moreover, under GO approximation the scattered ray fields follow the laws of reflection and refraction at a twomedia separation surface.

In order to derive the GO ray expression, one can resort to the Luneberg-Kline highfrequency expansion discussed in [13, 42, 43]. Alternatively, one can asymptotically evaluate the PO radiation integral, as discussed in $[44,45]$. This approach is the one used in this work. To start with, let us a surface $S$ illuminated by a generic incident field, $\vec{E}_{i}$, as shown in Fig. 4.1. Similar to what is discussed in the section about PO radiation integral, the scattered field $\vec{E}_{S}$ can be calculated from the radiation of the equivalent surface currents $\vec{J}_{S}$ and $\vec{M}_{S}$ in absence of the surface:

$$
\vec{E}_{S}(P)=j k \iint_{S}\left[\hat{r} \times \vec{M}_{S}(Q)+\zeta \hat{r} \times \hat{r} \times \vec{J}_{S}(Q)\right] \frac{e^{-j k r}}{4 \pi r} d S
$$

where $P$ is the observation point, $Q$ is the integration point on the surface, $Q_{0}$ is a reference 
point, $\vec{r}=\overrightarrow{Q P},|\vec{r}|=r$, and $\hat{r}=\vec{r} / r$. Here $k$ is the wavenumber in the homogenous medium where the equivalent currents radiate, and $\zeta$ is the impedance of the medium.

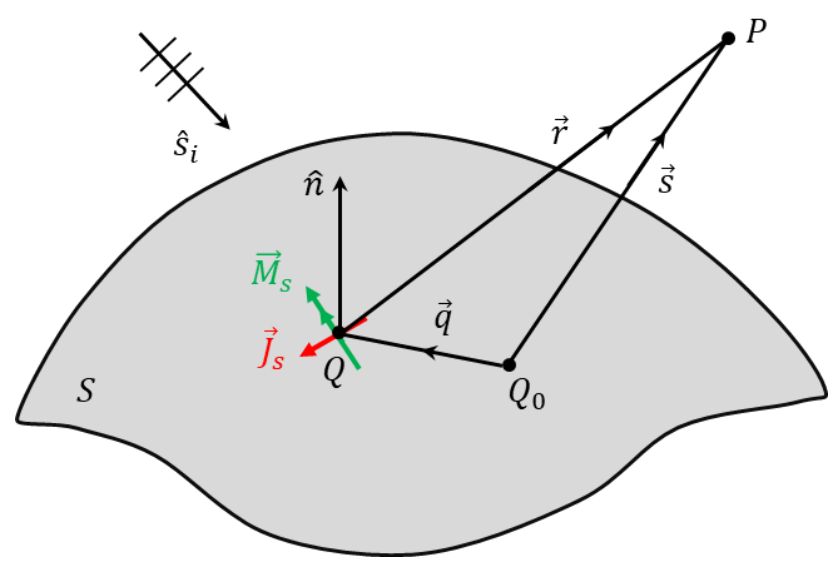

Figure 4.1: Field scattered by a surface $S$. The scattered field observed at a point $P$ is calculated from the radiation of the equivalent surface currents $\vec{J}_{s}$ and $\vec{M}_{s}$ estimated at an integration point $Q$ in the absence of the surface.

In the PO radiation integral, one can identify a slowly-varying function, $\vec{F}(Q)$, and a fastvarying function, $e^{-j k \Phi(Q)}$, as described in $[44,45]$ :

$$
\vec{E}_{S}(P)=\iint_{S} \vec{F}(Q) e^{-j k \Phi(Q)} d S
$$

If present, this integral is asymptotically dominated by a double stationary phase point. Therefore, one can asymptotically evaluate the integral in Eq. (4.2) by using the Method of Stationary Phase, as discussed in [44-46]. In particular, identifying the double stationary phase point with $Q_{0}$, the scattered field at $P$ can be evaluated by expending the integrand in Eq. (4.2) in the neighborhood of $Q_{0}$. The asymptotic integral evaluation leads to the following expression:

$$
\vec{E}_{S}(P) \simeq \frac{2 \pi \delta \vec{F}\left(Q_{0}\right) e^{-j k \Phi\left(Q_{0}\right)}}{j k \sqrt{\left|\operatorname{det}\left\{H_{\Phi}\left(Q_{0}\right)\right\}\right|}}
$$

where $H_{\Phi}\left(Q_{0}\right)$ is the Hessian matrix of the phase function $\Phi\left(Q_{0}\right)$, and $\delta$ can be $+1,-1$, or $j$, depending on the sign of the eigenvalues of $H_{\Phi}\left(Q_{0}\right)$ [44-46]. In the following, the scattered field is discussed for the case of GO reflection and transmission problems. 


\subsubsection{Reflection problem}

In the case of a $\mathrm{GO}$ reflection problem, the reference point $Q_{0}$ coincides with the point of reflection, $Q_{R}$. By applying Eq. (4.3) at $Q_{R}$ where the stationary phase condition is verified when the observation direction $\hat{s}$ coincides with the direction of the reflected ray $\hat{s}_{r}$, i.e. $\hat{s}=$ $\hat{s}_{r}$, the reflected field $\vec{E}_{r}(P)$ can be evaluated asymptotically as:

$$
\vec{E}_{r}(P) \simeq \vec{E}_{r}\left(Q_{R}\right) \sqrt{\frac{\rho_{1}^{r} \rho_{2}^{r}}{\left(\rho_{1}^{r}+s\right)\left(\rho_{2}^{r}+s\right)}} e^{-j k s}
$$

where $s$ is the distance along the ray path between the reflection point $Q_{R}$ and the observation point $P ; \rho_{1}^{r}$ and $\rho_{2}^{r}$ are the principal radii of curvature of the reflected wave front at the reflection point $Q_{R}$, and $\vec{E}_{r}\left(Q_{R}\right)$ is the reflected field at $Q_{R}$, which is described in Eq. (2.37).

In this work, the incident field is reflected by a $\mathrm{QO}$ surface and propagates to a FO sphere, as depicted in Fig. 4.2. The reflection point $Q_{R}$ is on the QO surface $S$ and the observation point $P$ is on the FO sphere, denoted as $Q_{F O}$. The distance along the reflected ray path is $s=$ $\left|\overrightarrow{Q_{R} Q_{F O}}\right|$. Consequently, one can express the $\mathrm{GO}$ reflected field as:

$$
\vec{E}_{G O}^{r}\left(Q_{F O}\right) \simeq \vec{E}_{r}\left(Q_{R}\right) \sqrt{\frac{\rho_{1}^{r} \rho_{2}^{r}}{\left(\rho_{1}^{r}+s\right)\left(\rho_{2}^{r}+s\right)}} e^{-j k s}
$$

where $s$ is explicitly derived in Appendix E.1, $\rho_{1}^{r}$ and $\rho_{2}^{r}$ are calculated in Appendix E.2.

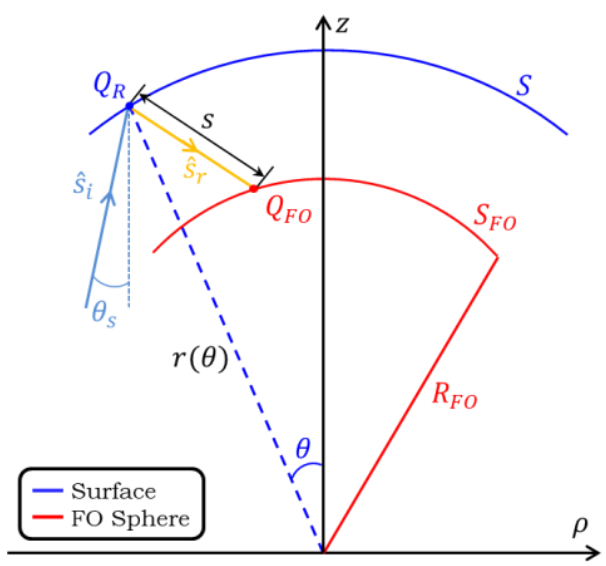

Figure 4.2: Geometry for a reflection problem with the description of the GO propagation. 


\subsubsection{Transmission problem}

For the transmission problem, similar to the reflection case, the reference point $Q_{0}$ coincides with the point of transmission, $Q_{T}$. By applying Eq. (4.3) at $Q_{T}$ where the stationary phase condition is verified when the observation direction $\hat{s}$ coincides with the direction of the transmitted ray $\hat{s}_{t}$, i.e. $\hat{s}=\hat{s}_{t}$, the GO transmitted field observed at $P$ can be asymptotically evaluated as:

$$
\vec{E}_{t}(P) \simeq \vec{E}_{t}\left(Q_{T}\right) \sqrt{\frac{\rho_{1}^{t} \rho_{2}^{t}}{\left(\rho_{1}^{t}+s\right)\left(\rho_{2}^{t}+s\right)}} e^{-j k s}
$$

where $\rho_{1}^{t}$ and $\rho_{2}^{t}$ are the principal radii of curvature of the transmitted wave front at the transmission point $Q_{T}$, and $\vec{E}_{t}\left(Q_{T}\right)$ is the transmitted field at $Q_{T}$, which is described in Eq. (2.39). In this work, $\vec{E}_{t}(P)$ is the GO transmitted field on the FO sphere that can be expressed as:

$$
\vec{E}_{G O}^{t}\left(Q_{F O}\right) \simeq \vec{E}_{t}\left(Q_{T}\right) \sqrt{\frac{\rho_{1}^{t} \rho_{2}^{t}}{\left(\rho_{1}^{t}+s\right)\left(\rho_{2}^{t}+s\right)}} e^{-j k s}
$$

The expressions of $\rho_{1}^{t}$ and $\rho_{2}^{t}$ are discussed in Appendix E.3.

From Eq. (4.5) and (4.7) one can see that when the GO field propagates along the ray path from the reference point $\left(Q_{R}, Q_{T}\right)$ towards the observation point $Q_{F O}$, its amplitude tends to attenuate (in case of diverging wave front) or to increase (in case of converging wave front) with a spreading factor, $\sqrt{\frac{\rho_{1} \rho_{2}}{\left(\rho_{1}+s\right)\left(\rho_{2}+s\right)}}$, and its phase changes along the propagation path as, $e^{-j k s}$ 


\subsection{Analytical GO fields}

In section 4.1, we know how to obtain GO fields by applying asymptotic evaluation; however, almost all characteristic parameters involved in the calculation need to be calculated numerically, which makes them not intuitive. For some simplified cases, it is possible to find analytical expressions for GO fields. In Eq. (4.5) and (4.7), we find that once we know the field at the reference point, the GO field can be obtained by considering the spreading factor and the phase variation.

We start with the broadside incidence. In subsection 4.2.1, we will discuss how we define the scattered field at a reference point, calculate the spreading factor, and solve the phase variation, for each QO component. After that in subsection 4.2.2, we consider the case of slightly off-broadside incidence. In this case, the analytical GO field is the same as the broadside one, but with a linear and coma phase term (for elliptical mirror also a compensation phase term).

\subsubsection{Broadside incidence}

In this part, we derive the analytical GO field for each QO component with broadside incidence. For the spreading factor, one can derive it by considering power budget among incident, reflected and transmitted rays, which is explicitly discussed in Appendix F and can be expressed as follows:

$$
\left\{\begin{array}{l}
P_{r}^{\perp / \|}=P_{i}^{\perp / \|}\left|\Gamma^{\perp / \|}\right|^{2} \\
P_{t}^{\perp / \|}=P_{i}^{\perp / \|}\left|\tau^{\perp / \|}\right|^{2} \frac{\zeta_{1}}{\zeta_{2}} \frac{\cos \theta_{t}}{\cos \theta_{i}}
\end{array}\right.
$$

where $P_{i}^{\perp / \|}, P_{r}^{\perp / \|}$, and $P_{t}^{\perp / \|}$ are incident, reflected and transmitted power, respectively. The incident ray propagates in the medium I (impedance $\zeta_{1}$ ) and is transmitted into the medium II (impedance $\zeta_{2}$ ). In a reflection problem, we use a PEC as the interface, i.e. there is no transmission and $\left|\Gamma^{\perp / \|}\right|=1$. Therefore, the incident power is equal to the reflected power, which agrees with the law of conservation of energy. 


\subsubsection{Parabolic reflector}

In the case of a parabolic reflector, a plane wave incident from broadside is shown in Fig. 4.3. It impinges on a point $Q_{R}$ on the surface $S$ and reflected to a point $Q_{F O}$ on the FO sphere $S_{F O}$. $\hat{s}_{i}$ and $\hat{s}_{r}$ represent the incident and reflected propagation unit vectors, respectively. $O$ indicates the position of the focus of the parabolic reflector, $Q_{a}$ is a point on the focal plane of the reflector, $f$ is the focal distance, and $r(\theta)$ is the radial distance between the reflector focus and its surface.

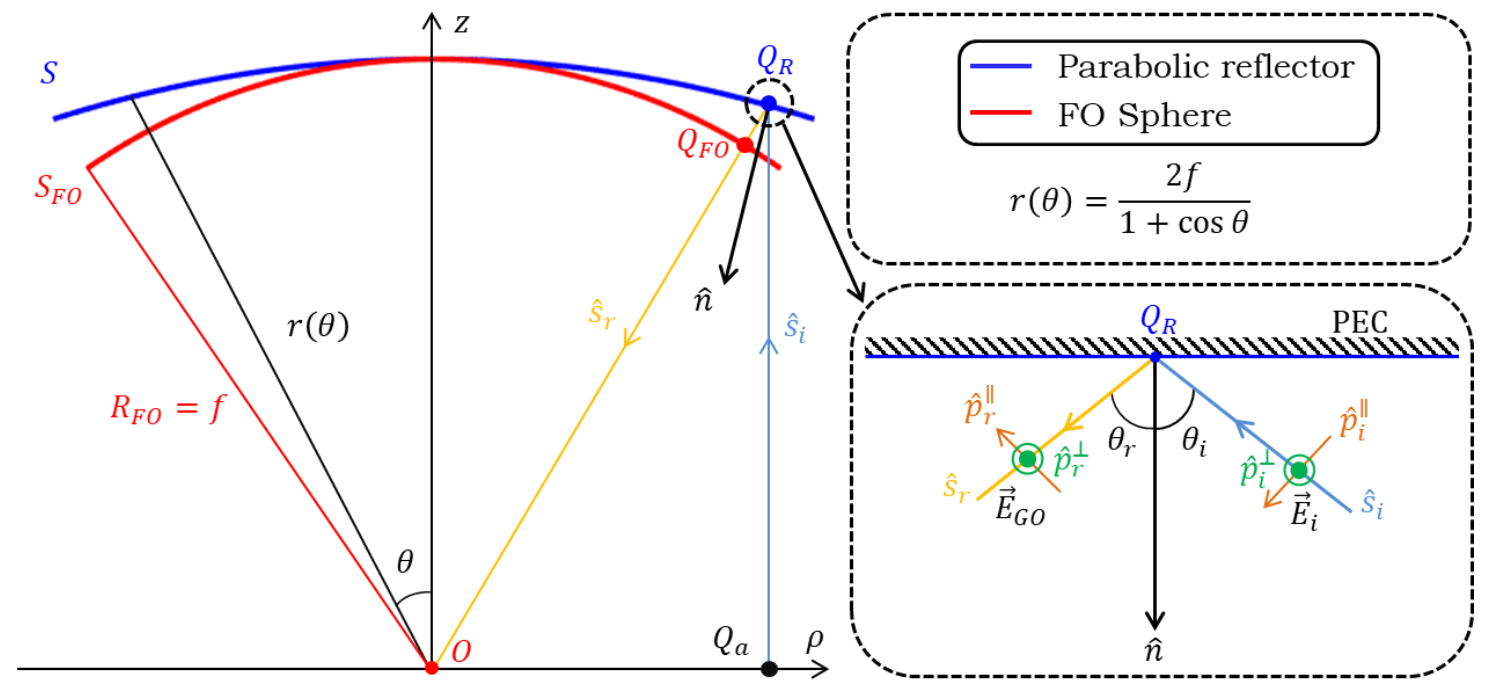

Figure 4.3: A parabolic reflector with broadside plane wave incidence.

Here we describe the incident plane wave by neglecting its phase term:

$$
\vec{E}_{i}=E_{0} \hat{p}_{i}
$$

where $E_{0}$ is the amplitude of the plane wave and $\hat{p}_{i}$ is the Ludwig-III polarization which is described in section 2.2.1. For broadside incidence $\left(\theta_{s}=0^{\circ}, \phi_{s}=0^{\circ}\right)$, the polarization can be simplified as below:

$$
\left\{\begin{array}{l}
\hat{p}_{i}=\hat{y}: \text { Co }- \text { Pol. } \\
\hat{p}_{i}=\hat{x}: \text { Cx }- \text { Pol. }
\end{array}\right.
$$

At $Q_{a}$, we decompose the incident field into perpendicular (TE) and parallel (TM) components:

$$
\vec{E}_{i}\left(Q_{a}\right)=E_{i}^{\perp}\left(Q_{a}\right) \hat{p}_{i}^{\perp}\left(Q_{a}\right)+E_{i}^{\|}\left(Q_{a}\right) \hat{p}_{i}^{\|}\left(Q_{a}\right)
$$

where $\hat{p}_{i}^{\perp}$ and $\hat{p}_{i}^{\|}$can be calculated by using the law of reflection with $\hat{s}_{i}=+\hat{z}$ :

$$
\left\{\begin{array}{l}
\hat{p}_{i}^{\perp}=\frac{\hat{s}_{i} \times \hat{n}}{\left|\hat{s}_{i} \times \hat{n}\right|}=-\hat{\phi} \\
\hat{p}_{i}^{\|}=\hat{p}_{i}^{\perp} \times \hat{s}_{i}=-\hat{\rho}
\end{array}\right.
$$

TE and TM electric fields are calculated as: 


$$
\left\{\begin{array}{l}
E_{i}^{\perp}\left(Q_{a}\right)=\vec{E}_{i} \cdot \hat{p}_{i}^{\perp}=-E_{0}\left(\hat{p}_{i} \cdot \hat{\phi}\right) \\
E_{i}^{\|}\left(Q_{a}\right)=\vec{E}_{i} \cdot \hat{p}_{i}^{\|}=-E_{0}\left(\hat{p}_{i} \cdot \hat{\rho}\right)
\end{array}\right.
$$

At $Q_{F O}$, the reflected field on the FO sphere, $\vec{E}_{G O}\left(Q_{F O}\right)$, can also be divided into TE and TM components:

$$
\vec{E}_{G O}\left(Q_{F O}\right)=E_{G O}^{\perp}\left(Q_{F O}\right) \hat{p}_{r}^{\perp}+E_{G O}^{\|}\left(Q_{F O}\right) \hat{p}_{r}^{\|}
$$

In the following, the calculation of the amplitude, phase, and polarization of $\vec{E}_{G O}$ is described.

\section{- Amplitude}

For a reflection problem, by considering the law of conservation of energy, we can relate the reflected power, $P_{r}^{\perp / \|}\left(Q_{F O}\right)$, to the incident power, $P_{i}^{\perp / \|}\left(Q_{a}\right)$, as follows:

$$
P_{r}^{\perp / \|}\left(Q_{F O}\right)=P_{i}^{\perp / \|}\left(Q_{a}\right)\left|\Gamma^{\perp / \|}\left(Q_{R}\right)\right|^{2}
$$

where $\left|\Gamma^{\perp / \|}\left(Q_{R}\right)\right|=1$ for a PEC (Fig. 4.3). By using this condition, one can derive the amplitude relation between the incident field and the GO field, i.e. the spreading factor, $S_{\text {pread }}$. For a parabolic reflector, $S_{\text {pread }}$ can be expressed as follows (Appendix G.1):

$$
S_{\text {pread }}(\theta)=\frac{\left|E_{G O}^{\perp / \|}\right|}{\left|\Gamma^{\perp / \|} E_{i}^{\perp / \|}\right|}=\frac{2}{1+\cos \theta}
$$

Therefore, the amplitude of $\vec{E}_{G O}$ is obtained:

$$
\left|E_{G O}^{\perp / \|}\right|=S_{\text {pread }}\left|\Gamma^{\perp / \|} E_{i}^{\perp / \|}\right|
$$

Notice that if we define the polarization unit vector ( $\hat{p}_{i}^{\perp / \|}$ and $\left.\hat{p}_{r}^{\perp / \|}\right)$ by using Eq. (4.12) and as shown in Fig. 4.3, for a PEC, $\Gamma^{\perp}=\Gamma^{\|}=-1$.

\section{- Phase}

The center of the coordinate system is defined at the focus of the parabola. Since $\left|Q_{R}-Q_{a}\right|+\left|Q_{R}-Q_{F O}\right|=f$, the GO field has a constant phase on the FO sphere. Therefore, we can neglect this phase term in $E_{G O}^{\perp / \|}$.

\section{- Polarization}

The polarizations of $\vec{E}_{G O}$ are derived as follows $\left(\hat{s}_{r}=-\hat{r}\right)$ :

$$
\left\{\begin{array}{l}
\hat{p}_{r}^{\perp}=\frac{\hat{s}_{r} \times \hat{n}}{\left|\hat{s}_{r} \times \hat{n}\right|}=-\hat{\phi} \\
\hat{p}_{r}^{\| \prime}=\hat{s}_{r} \times \hat{p}_{r}^{\perp}=-\hat{\theta}
\end{array}\right.
$$

By substituting (4.17) and (4.18) in (4.14), we can express $\vec{E}_{G O}$ analytically for broadside incidence:

$$
\vec{E}_{G O}=S_{\text {pread }}\left[\Gamma^{\| \prime}\left(\hat{p}_{i} \cdot \hat{\rho}\right) \hat{\theta}+\Gamma^{\perp}\left(\hat{p}_{i} \cdot \hat{\phi}\right) \hat{\phi}\right] E_{0}
$$




\subsubsection{Elliptical lens}

In the case of an elliptical lens, a plane wave incident from broadside is shown in Fig. 4.4. It impinges on a point $Q_{T}$ on the surface $S$ and transmitted to a point $Q_{F O}$ on the FO sphere $S_{F O}$. $\hat{s}_{i}$ and $\hat{s}_{t}$ are incident and transmitted propagation unit vectors, respectively. $O$ indicates the position of the lower focus of the lens, $Q_{a}$ is a point on the equivalent aperture of the lens, $R_{l}$ is the rim distance, and $r(\theta)$ is the radial distance from the lower focus to the surface.

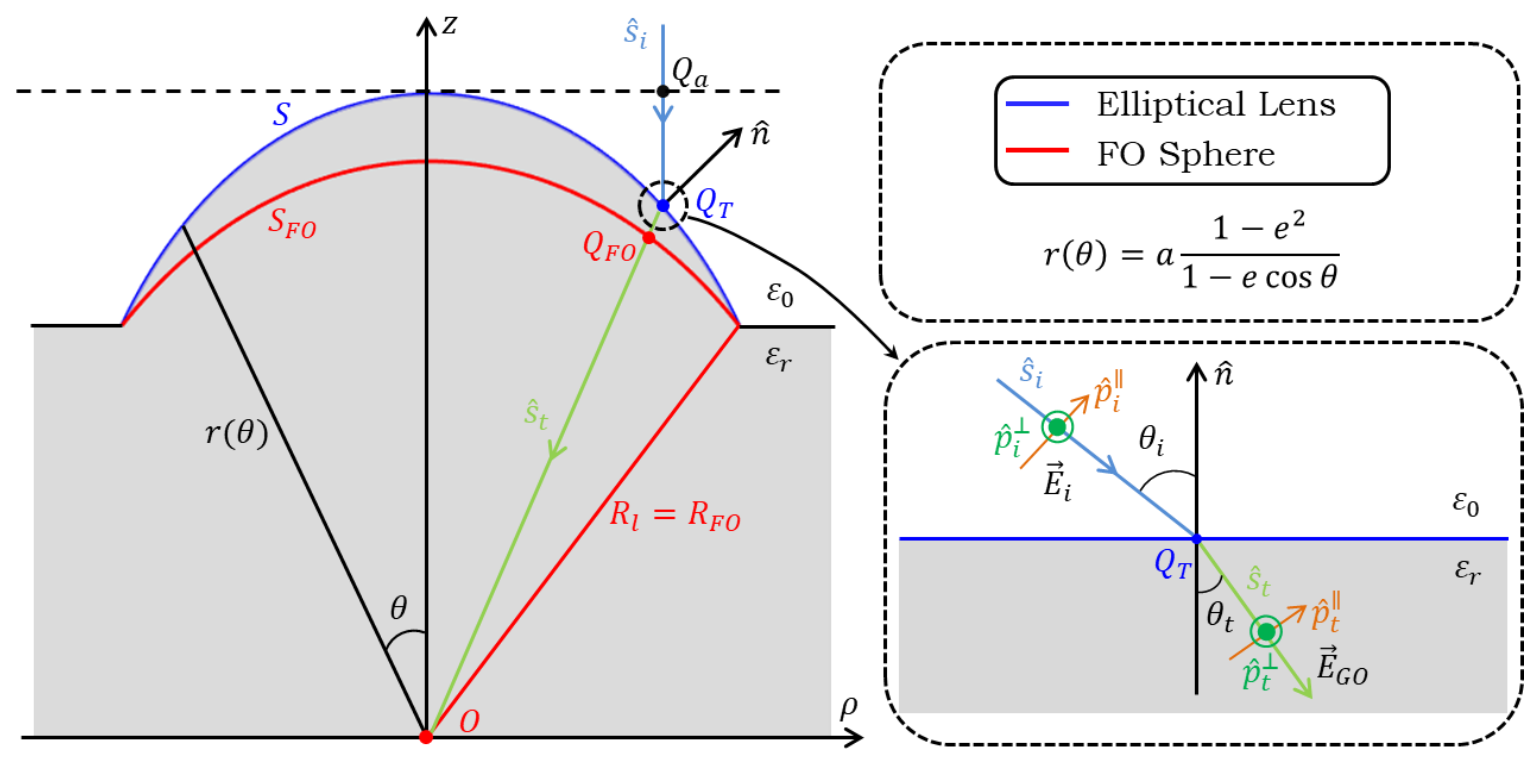

Figure 4.4: An elliptical lens with broadside plane wave incidence.

The incident plane wave is defined the same as Eq. (4.9). Similar to a parabolic reflector, at $Q_{a}$, we decompose the incident field into TE and TM components:

$$
\vec{E}_{i}\left(Q_{a}\right)=E_{i}^{\perp}\left(Q_{a}\right)(-\hat{\phi})+E_{i}^{\|}\left(Q_{a}\right) \hat{\rho}
$$

and at $Q_{F O}$ we decompose the GO field:

$$
\vec{E}_{G O}\left(Q_{F O}\right)=E_{G O}^{\perp}\left(Q_{F O}\right)(-\hat{\phi})+E_{G O}^{\|}\left(Q_{F O}\right) \hat{\theta}
$$

To calculate the amplitude, $\left|E_{G O}^{\perp / \|}\right|$, we need to consider the power budget for a transmission problem: The transmitted power, $P_{t}^{\perp / \|}\left(Q_{F O}\right)$, is related to the incident power, $P_{i}^{\perp / \|}\left(Q_{a}\right)$, as described in Eq. (4.8):

$$
P_{t}^{\perp / \|}\left(Q_{F O}\right)=P_{i}^{\perp / \|}\left(Q_{a}\right)\left|\tau^{\perp / \|}\left(Q_{T}\right)\right|^{2} \frac{\zeta_{0}}{\zeta_{d}} \frac{\cos \theta_{t}}{\cos \theta_{i}}
$$

where $\zeta_{0}$ is the impedance in free space, $\zeta_{d}=\zeta_{0} / \sqrt{\varepsilon_{r}}$ is the impedance of the medium, $\theta_{i}$ and $\theta_{t}$ are the incident and transmitted angles with respect to the normal vector of the surface (Fig. 
4.4), respectively, which are calculated in Appendix G.6. By solving Eq. (4.22), one can derive the spreading factor, which is explicitly derived in Appendix G.2 for an elliptical lens:

$$
S_{\text {pread }}(\theta)=\frac{\left|E_{G O}^{\perp / \|}\right|}{\left|\tau^{\perp / \|} E_{i}^{\perp / \|}\right|}=\frac{a\left(1-e^{2}\right)}{R_{l}(1-e \cos \theta)}
$$

where $a$ is the semi-major axis, $c$ is the focal distance, and $e=c / a$ is the eccentricity. Therefore, the amplitude of the GO field is obtained:

$$
\left|E_{G O}^{\perp / \|}\right|=S_{\text {pread }}\left|\tau^{\perp / \|} E_{i}^{\perp / \|}\right|
$$

The center of the coordinate system is defined at the lower focus of the lens. Since we choose the eccentricity of the lens to be $e=1 / \sqrt{\varepsilon_{r}}$, the GO field has a constant phase on the FO sphere. Therefore, we can neglect the phase term in $E_{G O}^{\perp / \|}$. By substituting Eq. (4.24) in Eq. (4.21), we can express $\vec{E}_{G O}$ analytically for broadside incidence:

$$
\vec{E}_{G O}=S_{\text {pread }}\left[\tau^{\|}\left(\hat{p}_{i} \cdot \hat{\rho}\right) \hat{\theta}+\tau^{\perp}\left(\hat{p}_{i} \cdot \hat{\phi}\right) \hat{\phi}\right] E_{0}
$$

\subsubsection{Hyperhemispherical lens}

We have introduced in section 2.1.3 that there are two types of hemispherical lenses. For the one with the extension length: $L / R_{s p h}=0.32-0.35$ [24], it is a good approximation of an elliptical silicon lens. Therefore, the GO field can be approximated by using Eq. (4.25). While for a hyperhemispherical lens, it is able to perfectly focalize a convergent incident wave on its focal plane [34]. Consequently, it is worth studying its analytical GO field for a broadside incidence.

A hyperhemispherical lens with the extension length $L=R_{s p h} / \sqrt{\varepsilon_{r}}$ is shown in Fig. 4.5. If the incident wave is a convergent spherical wave that converges at a virtual focus $O_{v}$ below the lens, illustrated as the blue colored rays, then geometrically the transmitted fields (green colored rays) will converge at the center of the base, $O$. It is discussed in [34] that the distance from the apex of the lens to the virtual focus is:

$$
F_{v}=R_{s p h}\left(\sqrt{\varepsilon_{r}}+1\right)
$$

The incident field at $Q_{T}$, i.e. $\vec{E}_{i}\left(Q_{T}\right)$, can be expressed as:

$$
\vec{E}_{i}\left(Q_{T}\right)=\left[E_{i}^{\perp}\left(Q_{T}\right)\left(-\hat{\phi}_{v}\right)+E_{i}^{\|}\left(Q_{T}\right) \hat{\theta}_{v}\right] \frac{1}{r_{v}}
$$

To derive the GO field, similar to an elliptical lens, we can first calculate the spreading factor (Appendix G.3): 


$$
S_{\text {pread }}(\theta)=\frac{\left|E_{G O}^{\perp / \|}\right|}{\left|\tau^{\perp / \|} E_{i}^{\perp / \|}\right|}=\frac{r(\theta)}{R_{F O}} \sqrt{\frac{\sin \theta}{\sin \theta_{v}} \frac{d \theta_{v}}{d \theta}} \sqrt{\frac{\cos \theta_{t}}{\cos \theta_{i}}}
$$

where $\theta_{v}$ is depicted in Fig. 4.5 and $d \theta_{v} / d \theta$ is calculated in Appendix G.6.

For the phase term, we define the center of the coordinate system at the base of the lens, $O$. The field transmitted into the lens and propagating on the FO sphere has a constant phase over the sphere. Therefore, we can neglect the phase term in $E_{G O}^{\perp / \|}$ and express $\vec{E}_{G O}$ analytically for broadside incidence as:

$$
\vec{E}_{G O}=S_{\text {pread }}\left[\tau^{\|}\left(\vec{E}_{i} \cdot \hat{\theta}_{v}\right) \hat{\theta}+\tau^{\perp}\left(\vec{E}_{i} \cdot \hat{\phi}_{v}\right) \hat{\phi}\right]
$$

where $\hat{\phi}_{v}=\hat{\phi}$. For a hyperhemispherical lens, we just investigate the broadside incidence. The slightly off-broadside incidence will be studied in the future.

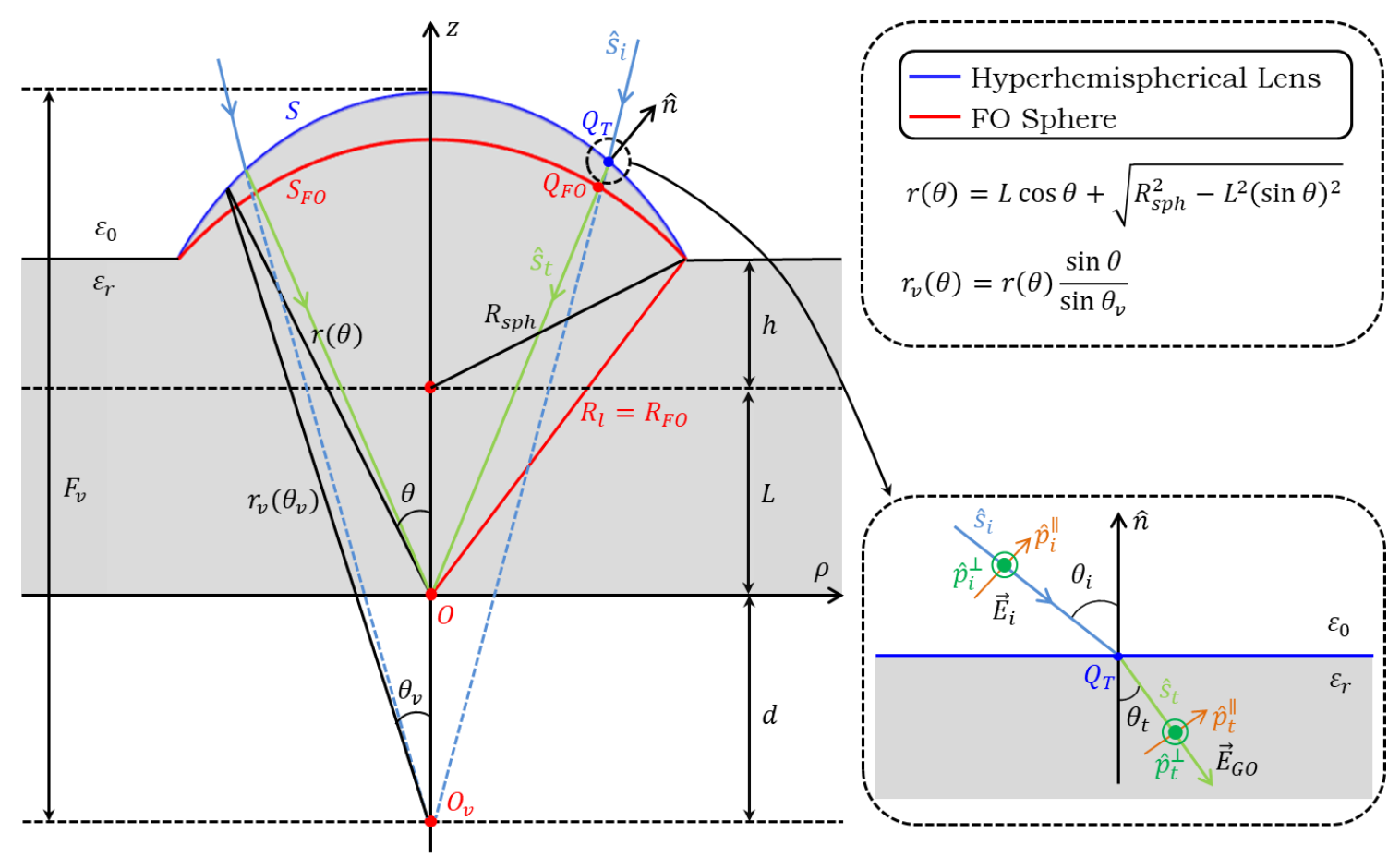

Figure 4.5: A hyperhemispherical lens with broadside incidence.

\subsubsection{Hyperbolic lens}

In the case of a hyperbolic lens, incident plane wave propagates in a medium $\left(\varepsilon_{r}\right)$ at broadside direction $\left(\hat{s}_{i}=-\hat{z}\right)$, as shown in Fig. 4.6. It impinges on $Q_{T}$ and transmitted to $Q_{F O} . O$ indicates the position of the lower focus of the hyperbola, and $Q_{a}$ is a point on the equivalent aperture of the hyperbolic lens. The incident plane wave is defined in Eq. (4.9), and it can be expressed at $Q_{a}$ as: 


$$
\vec{E}_{i}\left(Q_{a}\right)=E_{i}^{\perp}\left(Q_{a}\right)(-\hat{\phi})+E_{i}^{\|}\left(Q_{a}\right) \hat{\rho}
$$

Moreover, the GO field can be expressed as:

$$
\vec{E}_{G O}\left(Q_{F O}\right)=E_{G O}^{\perp}\left(Q_{F O}\right)(-\hat{\phi})+E_{G O}^{\|}\left(Q_{F O}\right) \hat{\theta}
$$

For the amplitude, $\left|E_{G O}^{\perp / \|}\right|$, we should consider the power budget between the incident power and the transmitted power:

$$
P_{t}^{\perp / \|}\left(Q_{F O}\right)=P_{i}^{\perp / \|}\left(Q_{a}\right)\left|\tau^{\perp / \|}\left(Q_{T}\right)\right|^{2} \frac{\zeta_{d}}{\zeta_{0}} \frac{\cos \theta_{t}}{\cos \theta_{i}}
$$

By solving Eq. (4.32) we can derive the spreading factor (Appendix G.4):

$$
S_{\text {pread }}(\theta)=\frac{\left|E_{G O}^{\perp / \|}\right|}{\left|\tau^{\perp / \|} E_{i}^{\perp / \|}\right|}=\frac{1-e}{1-e \cos \theta}
$$

For the phase term, we define the center of the coordinate system at the lower focus of the hyperbola. Since we choose the eccentricity of the hyperbolic lens to be $e=\sqrt{\varepsilon_{r}}$, the GO field has constant phase on the FO sphere. Therefore, we can neglect the phase term and express $\vec{E}_{G O}$ analytically for broadside incidence as:

$$
\vec{E}_{G O}=S_{\text {pread }}\left[\tau^{\|}\left(\hat{p}_{i} \cdot \hat{\rho}\right) \hat{\theta}+\tau^{\perp}\left(\hat{p}_{i} \cdot \hat{\phi}\right) \hat{\phi}\right] E_{0}
$$

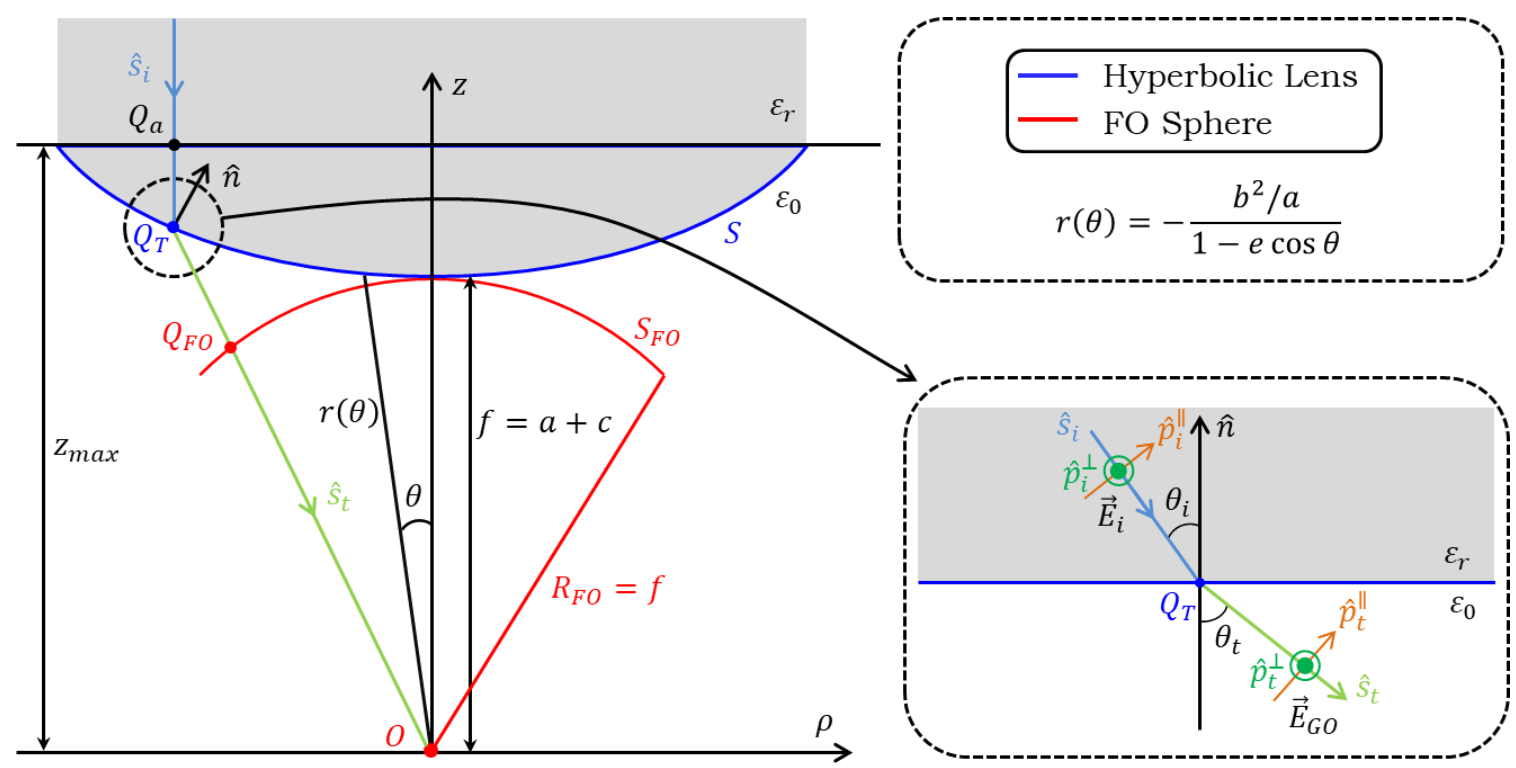

Figure 4.6: A hyperbolic lens with broadside plane wave incidence.

\subsubsection{Elliptical Mirror}

For an elliptical mirror, incident ray is a spherical wave emtted from a point source, as is shown in Fig. 4.7. It impinges on $Q_{R}$ and reflected to $Q_{F O}$. Since the source can be placed at either the upper focus, $O_{1}$, or the lower focus, $O_{2}$, we discuss two different cases: 


\section{- Case 1: Source placed at the upper focus, $O_{1}$}

In this case (Fig. 4.7), we can decompose the spherical incident wave at $Q_{R}$ as follows:

$$
\vec{E}_{i}\left(Q_{R}\right)=\left(E_{i}^{\perp}\left(Q_{R}\right) \hat{\phi}_{1}+E_{i}^{\|}\left(Q_{R}\right) \hat{\theta}_{1}\right) \frac{1}{r_{1}}
$$

Moreover, the GO field can be expressed as:

$$
\vec{E}_{G O}\left(Q_{F O}\right)=E_{G O}^{\perp}\left(Q_{F O}\right) \hat{\phi}_{2}+E_{G O}^{\|}\left(Q_{F O}\right) \hat{\theta}_{2}
$$

where $\hat{\phi}_{1}=\hat{\phi}_{2}$. Considering the law of conservation of energy and following the same steps described in the case of the parabolic reflector, we can express the spreading factor as follows (explicit derivation is listed in Appendix G.5):

$$
S_{\text {pread }}\left(\theta_{2}\right)=\frac{\left|E_{G O}^{\perp / \|}\right|}{\left|\Gamma^{\perp / \|} E_{i}^{\perp / \|}\right|}=\frac{\sqrt{r_{1}\left(\theta_{1}\right) r_{2}\left(\theta_{2}\right)}}{R_{F O}} \sqrt{\frac{a\left(1-e^{2}\right)}{2 c\left(e-\cos \theta_{2}\right)+a\left(1-e^{2}\right)}}
$$

where $r_{1}$ and $r_{2}$ are radial distances depicted in Fig. 4.7, $a$ is the semi-major axis, $c$ is the focal distance, and $e=c / a$ is the eccentricity.

For the phase term, we define the center of the coordinate system at the lower focus of the ellipse. Since $\left|Q_{R}-O_{1}\right|+\left|Q_{R}-Q_{F O}\right|=2 a-R_{F O}$, the GO field has a constant phase on the FO sphere. Therefore, we can express $\vec{E}_{G O}$ analytically for broadside incidence:

$$
\vec{E}_{G O}=S_{\text {pread }}\left[\Gamma^{\|}\left(\vec{E}_{i} \cdot \hat{\theta}_{1}\right) \hat{\theta}_{2}+\Gamma^{\perp}\left(\vec{E}_{i} \cdot \hat{\phi}_{1}\right) \hat{\phi}_{2}\right]
$$

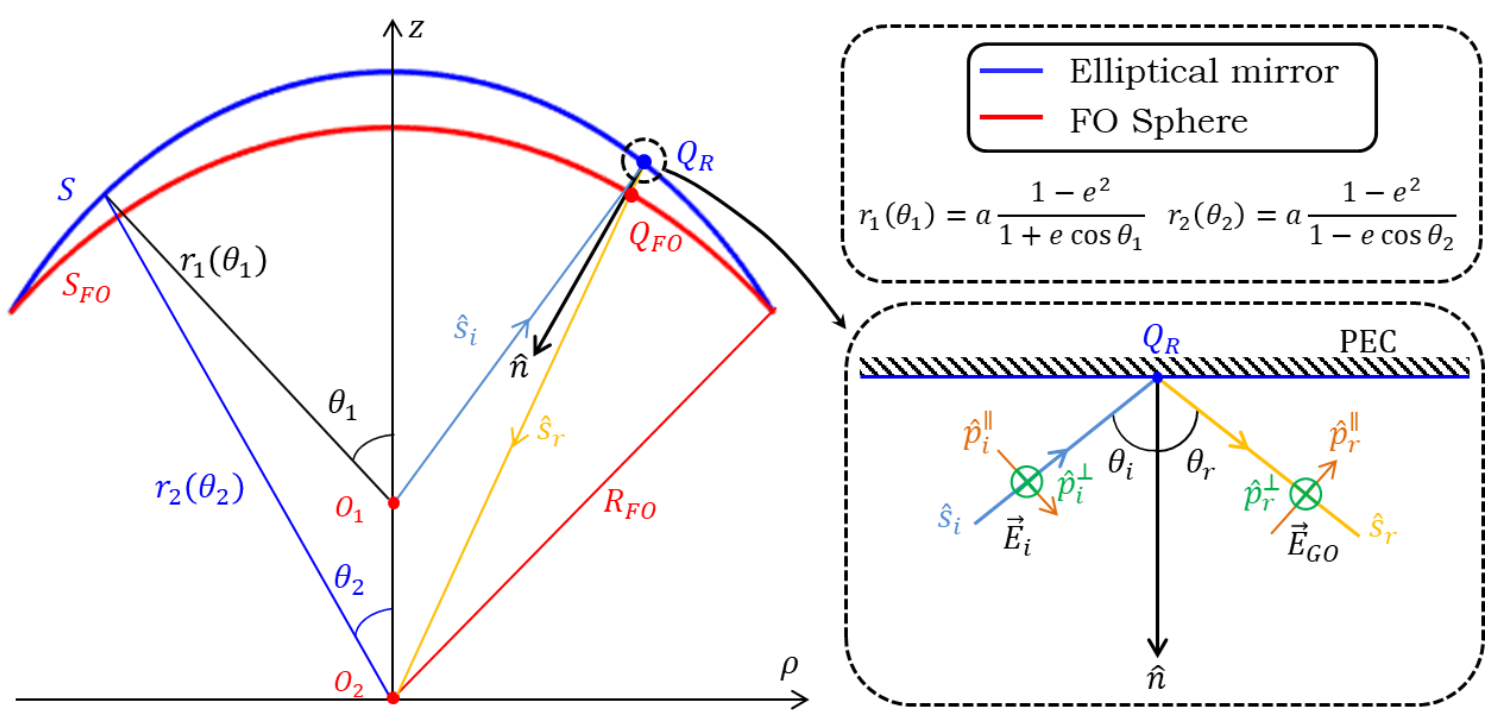

Figure 4.7: An elliptical mirror with broadside incidence. Source is placed at $O_{1}$. 


\section{- Case 2: Source placed at the lower focus, $O_{2}$}

In this case (Fig. 4.8), the incident wave is defined as follows:

$$
\vec{E}_{i}\left(Q_{R}\right)=\left[E_{i}^{\perp}\left(Q_{R}\right)\left(-\hat{\phi}_{2}\right)+E_{i}^{\|}\left(Q_{R}\right)\left(-\hat{\theta}_{2}\right)\right] \frac{1}{r_{2}}
$$

and the GO field is:

$$
\vec{E}_{G O}\left(Q_{F O}\right)=E_{G O}^{\perp}\left(Q_{F O}\right)\left(-\hat{\phi}_{1}\right)+E_{G O}^{\|}\left(Q_{F O}\right)\left(-\hat{\theta}_{1}\right)
$$

The spreading factor is obtained as follows:

$$
S_{\text {pread }}\left(\theta_{1}\right)=\frac{\left|E_{G O}^{\perp / \|}\right|}{\left|\Gamma^{\perp / \|} E_{i}^{\perp / \|}\right|}=\frac{\sqrt{r_{1}\left(\theta_{1}\right) r_{2}\left(\theta_{2}\right)}}{R_{F O}} \sqrt{\frac{a\left(1-e^{2}\right)}{2 c\left(e+\cos \theta_{1}\right)+a\left(1-e^{2}\right)}}
$$

For the phase term, the center of the coordinate system is defined at the upper focus of the ellipse, and it is still the case that the phase of the GO field is constant. Therefore, we can neglect the phase term and express the GO field as follows:

$$
\vec{E}_{G O}=S_{\text {pread }}\left[\Gamma^{\|}\left(\vec{E}_{i} \cdot \hat{\theta}_{2}\right) \hat{\theta}_{1}+\Gamma^{\perp}\left(\vec{E}_{i} \cdot \hat{\phi}_{2}\right) \hat{\phi}_{1}\right]
$$

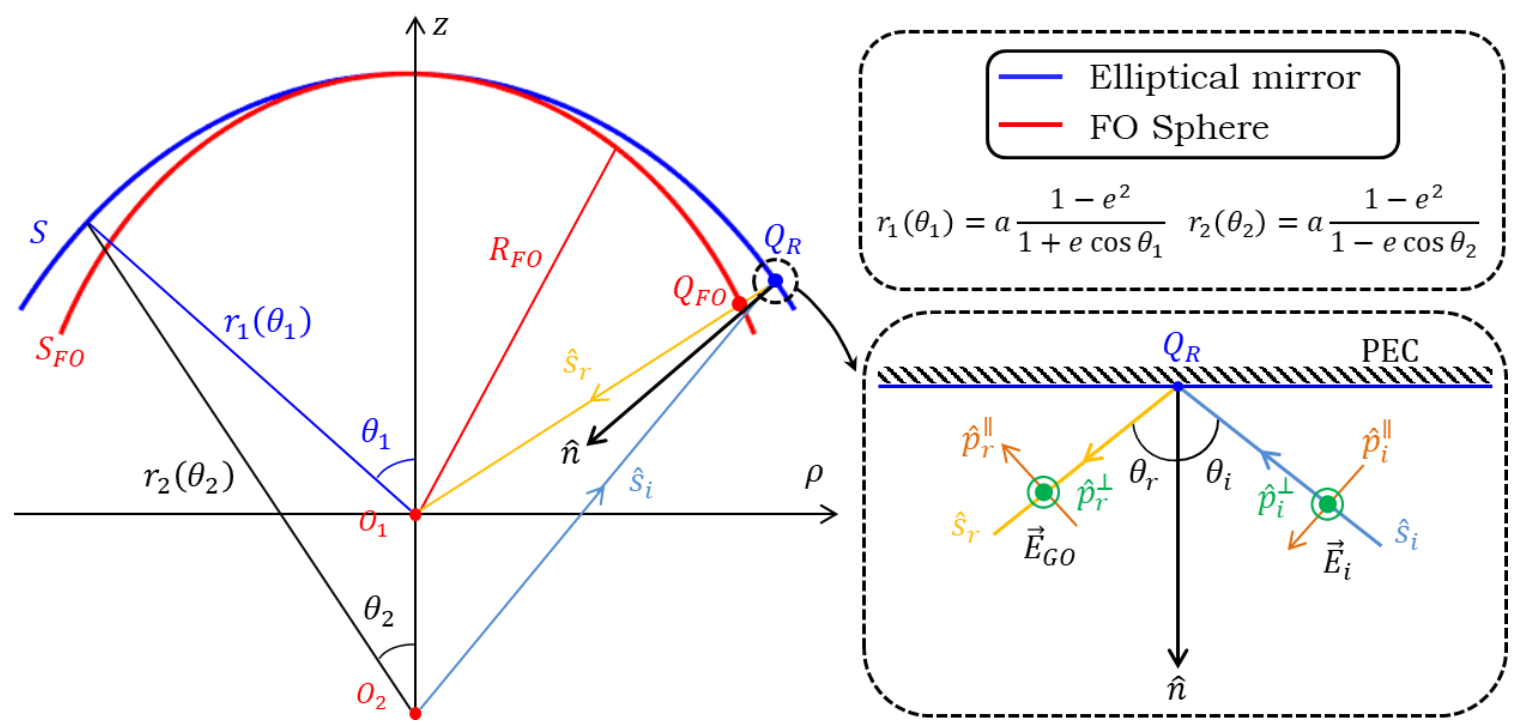

Figure 4.8: An elliptical mirror with broadside incidence. Source is placed at $\mathrm{O}_{2}$. 


\subsubsection{Comparison between GO ray fields and analytical GO fields}

Both GO ray fields and analytical GO fields have been derived in the preceding subsections. In this subsection, we compare these GO fields and check if the analytic GO fields are in good agreement with the GO ray fields. Here we use low f-number configurations because they are more challenging.

The preceding analytical expressions for GO fields are derived for broadside incidence. Moreover, phase information is neglected since the analytical GO fields have constant phase on FO spheres. Therefore, here we consider broadside incidence and compare only the amplitude of GO fields. The amplitude is decomposed into $\left|E_{\theta}\right|$ and $\left|E_{\phi}\right|$ components and the relative error is calculated for comparison:

$$
\varepsilon_{\theta / \phi}^{a m p}=\left|\frac{\left|E_{\theta / \phi}^{F O}\right|-\left|E_{\theta / \phi}^{G O}\right|}{\left|E_{\theta / \phi}^{G O}\right|}\right| \cdot 100 \%
$$

where $\left|E_{\theta / \phi}^{F O}\right|$ and $\left|E_{\theta / \phi}^{G O}\right|$ represent the amplitude of analytical GO fields and GO ray fields, respectively.

\section{- Parabolic reflector}

A parabolic reflector with the diameter of $D_{r}=100 \lambda_{0}\left(f_{0}=300 \mathrm{GHz}\right)$ and the f-number of $f_{\#}^{r}=0.6$ is illuminated by a unitary TM polarized plane wave incoming from the broadside.

Fig. 4.9 shows the relative amplitude errors of $\left|E_{\theta}\right|$ (Fig. 4.9a) and $\left|E_{\phi}\right|$ (Fig. 4.9b) components. As it can be seen, the error is extremely small, meaning the analytical GO field is in excellent agreement with the GO ray field.

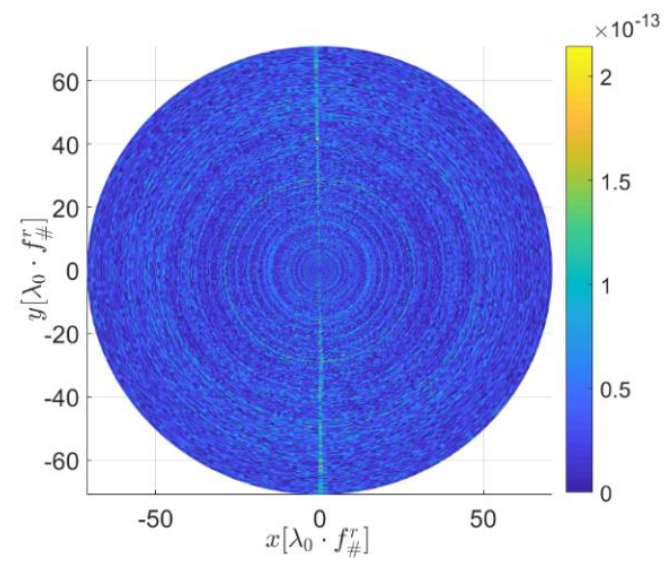

(a)

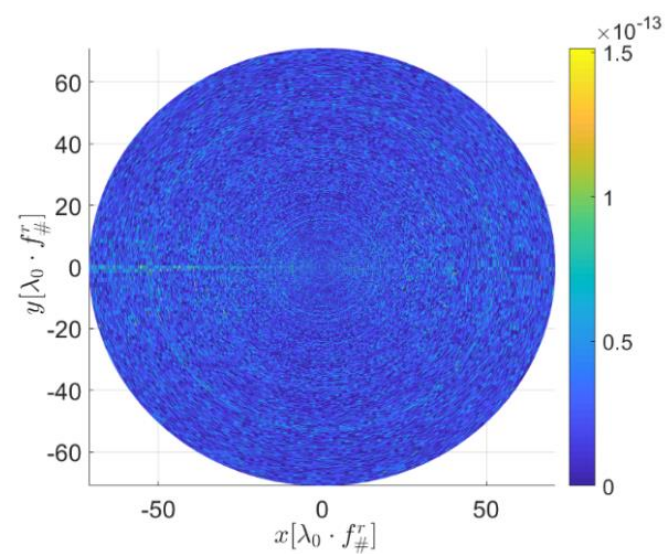

(b)

Figure 4.9: Relative amplitude errors of the GO fields: (a) $\left|E_{\theta}\right|$ component. (b) $\left|E_{\phi}\right|$ component, for a parabolic reflector with $D_{r}=100 \lambda_{0}\left(f_{0}=300 \mathrm{GHz}\right)$ and $f_{\#}^{r}=0.6$, illuminated by a unitary TM polarized plane wave incoming from the broadside. 


\section{- Elliptical lens}

Here we consider an elliptical silicon $\left(\varepsilon_{r}=11.9\right)$ lens applied with a matching layer. A quarter-wavelength matching layer at $300 \mathrm{GHz}$ made of Parylene $\left(\varepsilon_{m}=2.62\right)$ is applied, and the lens has the diameter of $D_{l}=5 \lambda_{0}\left(f_{0}=300 \mathrm{GHz}\right)$ and the f-number of $f_{\#}^{l}=0.6$, illuminated by a unitary TE polarized plane wave in broadside. Fig. 4.10 shows the relative amplitude errors of $\left|E_{\theta}\right|$ (Fig. 4.10a) and $\left|E_{\phi}\right|$ (Fig. 4.10b) components. As it can be seen, the analytical GO field is in excellent agreement with the GO ray field.

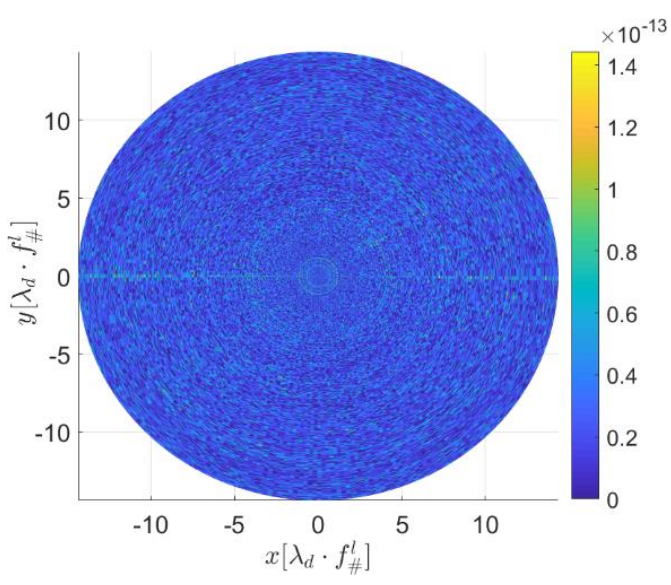

(a)

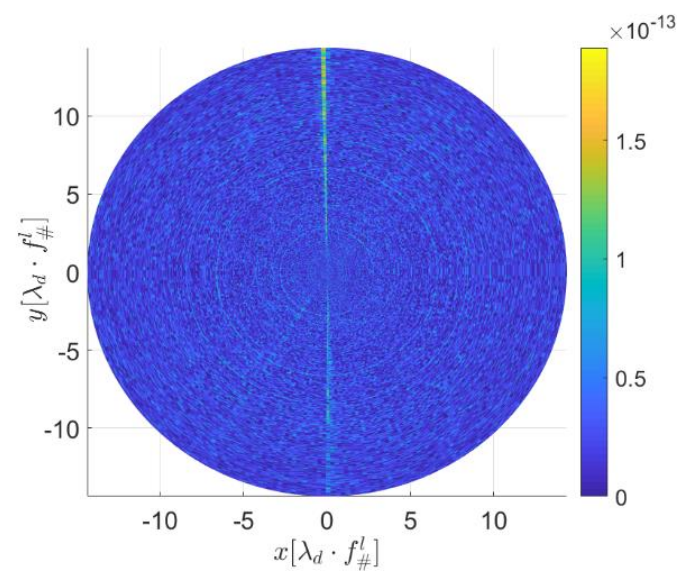

(b)

Figure 4.10: Relative amplitude errors of the GO fields: (a) $\left|E_{\theta}\right|$ component. (b) $\left|E_{\phi}\right|$ component, for an elliptical silicon $\left(\varepsilon_{r}=11.9\right)$ lens with $D_{l}=5 \lambda_{0}\left(f_{0}=300 \mathrm{GHz}\right)$ and $f_{\#}^{l}=0.6$, illuminated by a unitary TE polarized plane wave incoming from the broadside. A quarter-wavelength matching layer at $300 \mathrm{GHz}$ made of Parylene $\left(\varepsilon_{m}=\right.$ 2.62) is applied.

\section{- Hyperhemispherical lens}

A hyperhemispherical silicon $\left(\varepsilon_{r}=11.9\right)$ lens applied with a matching layer is introduced here. A quarter-wavelength matching layer at $300 \mathrm{GHz}$ made of Parylene $\left(\varepsilon_{m}=2.62\right)$ is applied, and the lens has $D_{h l}=5 \lambda_{0}\left(f_{0}=300 \mathrm{GHz}\right), f_{\#}^{h l}=0.6, R_{s p h}=2.66 \lambda_{0}$, and $L=$ $0.29 R_{s p h}$, illuminated by a unitary x-polarized convergent wave incident from the broadside. Fig. 4.11 shows the relative amplitude errors and it can be seen from the figure that the analytical GO field is almost the same as the GO ray field. 


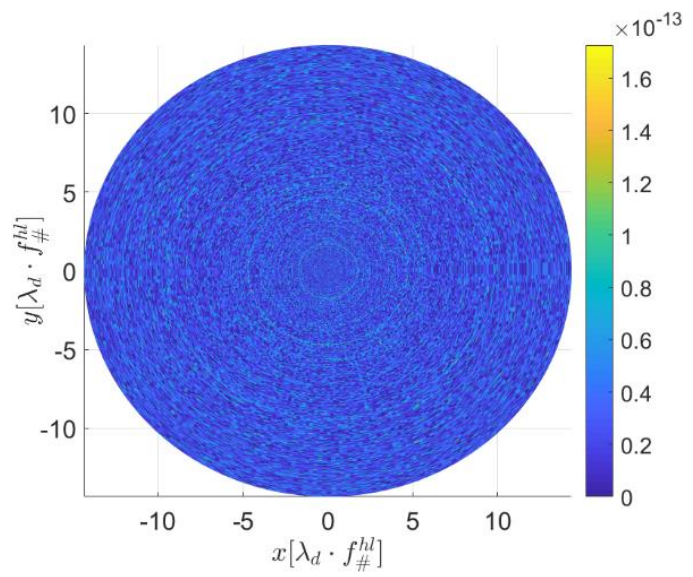

(a)

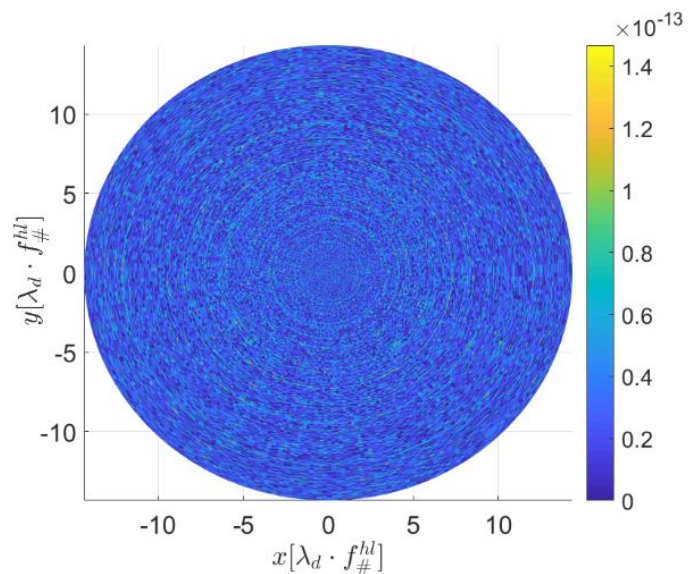

(b)

Figure 4.11: Relative amplitude errors of the GO fields: (a) $\left|E_{\theta}\right|$ component. (b) $\left|E_{\phi}\right|$ component, for a hyperhemispherical silicon $\left(\varepsilon_{r}=11.9\right)$ lens with $D_{h l}=5 \lambda_{0}\left(f_{0}=300 \mathrm{GHz}\right), f_{\#}^{h l}=0.6, R_{s p h}=2.66 \lambda_{0}$, and $L=0.29 R_{s p h}$, illuminated by a unitary x-polarized convergent wave incoming from the broadside. A quarterwavelength matching layer at $300 \mathrm{GHz}$ made of Parylene $\left(\varepsilon_{m}=2.62\right)$ is applied.

\section{- Hyperbolic lens}

Here we consider a hyperbolic plastic $\left(\varepsilon_{r}=2\right)$ lens with $D_{h}=100 \lambda_{0}\left(f_{0}=300 \mathrm{GHz}\right)$ and $f_{\#}^{h}=0.6$, illuminated by a unitary TE polarized plane wave incoming from the broadside. Fig. 4.12 shows the relative amplitude errors and it can be seen from the figure that the analytical GO field is in excellent agreement with the GO ray field.

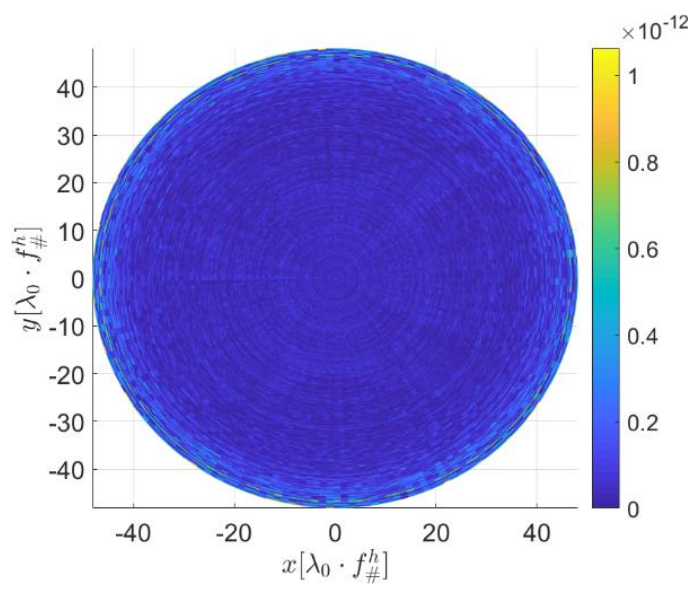

(a)

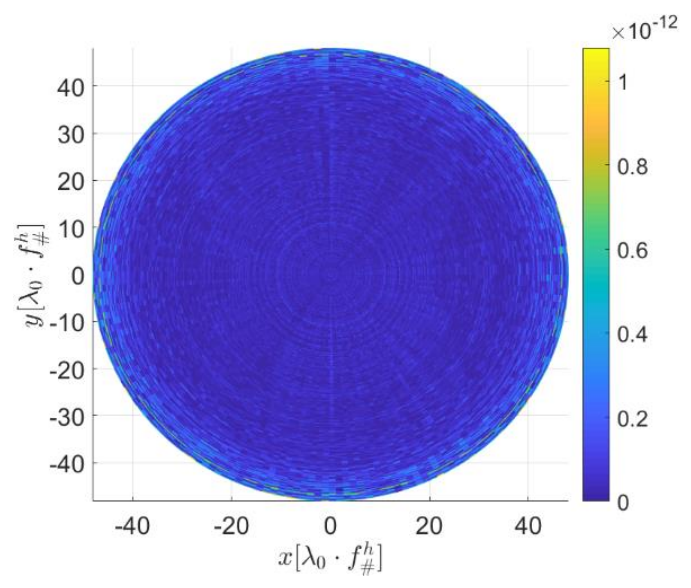

(b)

Figure 4.12: Relative amplitude errors of the GO fields: (a) $\left|E_{\theta}\right|$ component. (b) $\left|E_{\phi}\right|$ component, for a hyperbolic plastic $\left(\varepsilon_{r}=2\right)$ lens with $D_{h}=100 \lambda_{0}\left(f_{0}=300 \mathrm{GHz}\right)$ and $f_{\#}^{h}=0.6$, illuminated by a unitary TE polarized plane wave incoming from the broadside. 


\section{- Elliptical mirror}

For an elliptical mirror, we consider the case when a source is placed at the lower focus. The source is a unitary Huygens source with the electric current oriented along $\hat{x}$. The mirror has the diameter of $D_{m}=100 \lambda_{0}\left(f_{0}=300 \mathrm{GHz}\right)$, the semi-major axis of $a=75 \lambda_{0}$, and the focal distance of $c=15 \lambda_{0}$, i.e. the eccentricity is $e=0.2$ and the f-number is $f_{\#}^{m}=0.6$. Fig. 4.13 shows the relative amplitude errors of $\left|E_{\theta}\right|$ (Fig. 4.13a) and $\left|E_{\phi}\right|$ (Fig. 4.13b) components. As it can be seen, the error is very small. Therefore, the analytical GO field can be assumed the same as the GO ray field.

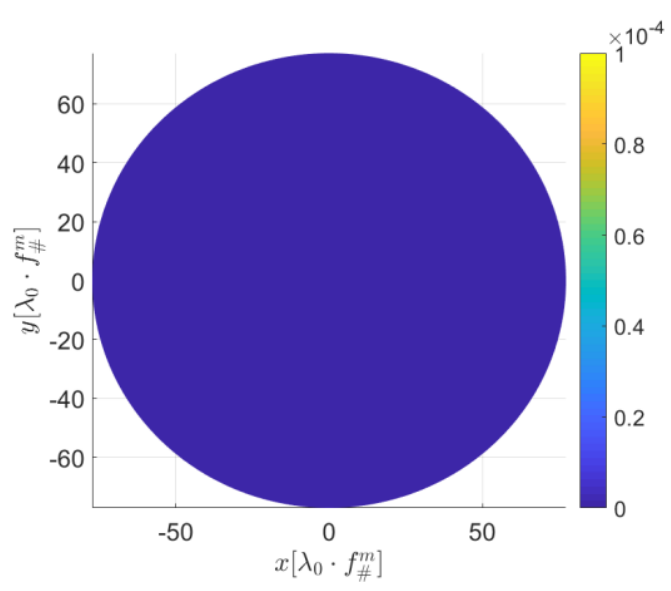

(a)

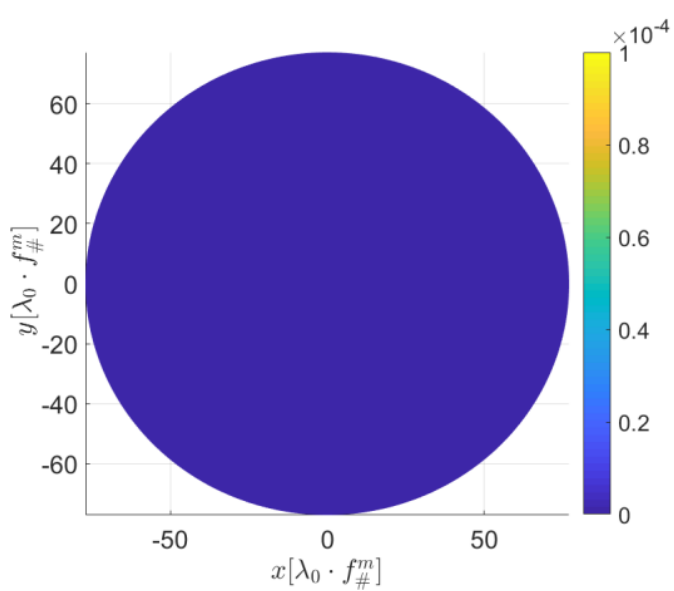

(b)

Figure 4.13: Relative amplitude errors of the GO fields: (a) $\left|E_{\theta}\right|$ component. (b) $\left|E_{\phi}\right|$ component, for an elliptical mirror with $D_{m}=100 \lambda_{0}\left(f_{0}=300 \mathrm{GHz}\right), f_{\#}^{m}=0.6$, and $e=0.2$, illuminated by a Huygens source placed at the lower focus with the electric current oriented along $\hat{x}$.

\section{- Conclusion}

To conclude, for all QO components with low f-number configurations, the analytical GO fields are in excellent agreement with the GO ray fields for broadside incidence. Therefore, we can assume the analytical GO fields are accurate approximations of the GO ray fields for broadside incidence. 


\subsubsection{Slightly off-broadside incidence}

In this subsection, we consider the analytical GO field for a slightly off-broadside incidence. When an external source is incident from a direction slightly off broadside, $\left(\theta_{s}, \phi_{s}\right)$, we introduce a key simplifying hypothesis to the incident field that the polarization of the incident field remains the same as the one in broadside case, while a progressive phase shift is added:

$$
\vec{E}_{i}\left(\theta_{s}\right) \simeq \vec{E}_{i}\left(\theta_{s}=0\right) \mathrm{e}^{-j \Phi}
$$

where $\Phi$ is the progressive phase shift.

The approximation in Eq. (4.44) corresponds to neglect the z-direction polarization vector which is proportional to $\tan \theta_{s}$. If we set the vector error as $20 \%$, meaning $\tan \theta_{s}<0.2$, we can calculate the angular limitation: $\theta_{s} \leq 11^{\circ}$. We have discussed in section 4.1 that the field on the FO sphere is evaluated by resorting to GO propagation of the incident field. Therefore, by implementing the approximation in Eq. (4.44), the analytical GO field can also be approximated by the one for broadside illumination (derived analytically in section 4.2.1), multiplied by the progressive phase shift:

$$
\vec{E}_{G O}\left(\theta_{S}\right) \simeq \vec{E}_{G O}\left(\theta_{s}=0\right) \mathrm{e}^{-j \Phi}
$$

The incident source can be a plane wave or a point source; therefore, we discuss two cases in the following.

\subsubsection{Plane wave incidence}

For a plane wave incidence, the progressive phase can be defined as follows:

$$
\Phi_{p w}=\vec{k}_{\rho, s} \cdot \vec{\rho}
$$

where $\vec{k}_{\rho, s}=k \hat{k}_{\rho, s}=k \sin \theta_{s}\left(\cos \phi_{s} \hat{x}+\sin \phi_{s} \hat{y}\right), k$ is the propagation constant in the medium, $\vec{\rho}=r(\theta) \sin \theta \hat{\rho}, \hat{\rho}=\cos \phi \hat{x}+\sin \phi \hat{y}$, and $r(\theta)$ is the radial distance of the surface $S$ shown in Fig. 4.14. 


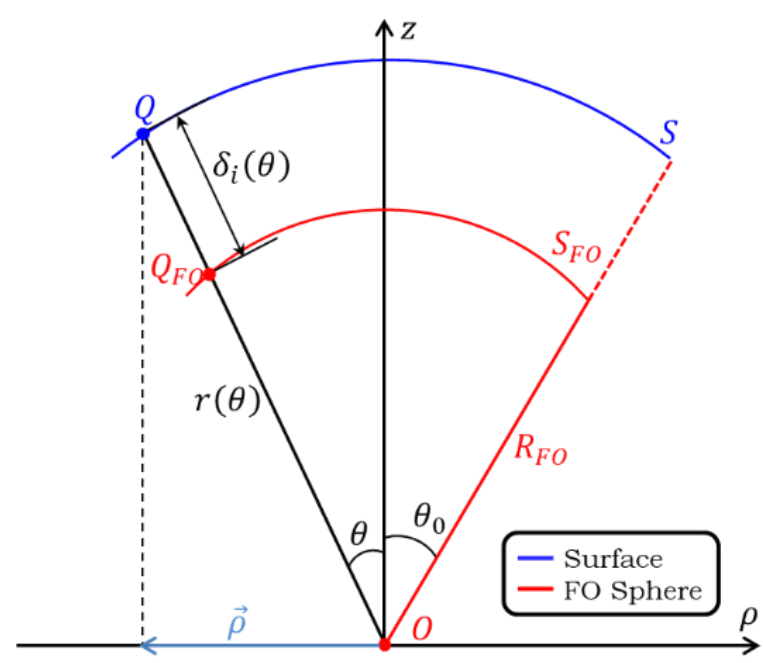

Figure 4.14: Geometry for the description of evaluating the progressive phase shift for slightly off-broadside plane wave incidence.

By substituting Eq. (4.46) in Eq. (4.45):

$$
\vec{E}_{G O}\left(\vec{k}_{\rho, s}\right) \simeq \vec{E}_{G O}\left(\vec{k}_{\rho, s}=0\right) \mathrm{e}^{-j \vec{k}_{\rho, s} \cdot \vec{\rho}}
$$

It can be seen in Fig. 4.14 that $r(\theta)=R_{F O}+\delta_{i}(\theta)$, where $\delta_{i}(\theta)$ is the distance between $Q_{F O}$ and $Q$. Therefore, we can express the vector $\vec{\rho}$ as follows:

$$
\vec{\rho}=\left(R_{F O}+\delta_{i}(\theta)\right) \sin \theta \hat{\rho}
$$

and calculate the term $\vec{k}_{\rho, s} \cdot \vec{\rho}$ :

$$
\begin{gathered}
\vec{k}_{\rho, s} \cdot \vec{\rho}=R_{F O} \frac{\vec{k}_{\rho, s}}{k}\left(1+\frac{\delta_{i}(\theta)}{R_{F O}}\right) \cdot k \sin \theta \hat{\rho} \\
\Rightarrow \vec{k}_{\rho, s} \cdot \vec{\rho}=\vec{\rho}_{f p}\left(1+\delta_{n}(\theta)\right) \cdot \vec{k}_{\rho}
\end{gathered}
$$

where $\vec{\rho}_{f p}$ is called flash point that is defined as:

$$
\vec{\rho}_{f p}=R_{F O} \frac{\vec{k}_{\rho, s}}{k}
$$

and $\delta_{n}(\theta)$ is the distance defined as:

$$
\delta_{n}(\theta)=\frac{\delta_{i}(\theta)}{R_{F O}}
$$

By substituting Eq. (4.49) in Eq. (4.47):

$$
\vec{E}_{G O}\left(\vec{k}_{\rho, s}\right) \approx \vec{E}_{G O}\left(\vec{k}_{\rho, s}=0\right) \mathrm{e}^{-j \vec{k}_{\rho} \cdot \vec{\rho}_{f p}} e^{-j \vec{k}_{\rho} \cdot \vec{\rho}_{f p} \delta_{n}(\theta)}
$$

In conclusion, for different QO components, as long as we calculate the flash point $\vec{\rho}_{f p}$ and the distance $\delta_{n}(\theta)$ within the defined applicability region, we can approximate the analytical GO field by the one defined for the broadside illumination multiplied by two phase terms. The 
first phase term in Eq. (4.52), $\mathrm{e}^{-j \vec{k}_{\rho} \cdot \vec{\rho}_{f p}}$, indicates a linear phase shift; while the second term, $e^{-j \vec{k}_{\rho} \cdot \vec{\rho}_{f p} \delta_{n}(\theta)}$, represents a coma phase. To better explain $\vec{\rho}_{f p}, \delta_{n}(\theta)$ and these phase terms, we take a parabolic reflector as an example:

\section{- Parabolic reflector}

For a parabolic reflector, $\delta_{n}(\theta)$ is calculated as follows:

$$
\delta_{n}(\theta)=\frac{r(\theta)-R_{F O}}{R_{F O}}=\frac{1-\cos \theta}{1+\cos \theta}
$$

and the flash point $\vec{\rho}_{f p}$ is:

$$
\vec{\rho}_{f p}=R_{F O} \frac{\vec{k}_{\rho, s}}{k_{0}}=f \sin \theta_{s}\left(\cos \phi_{s} \hat{x}+\sin \phi_{s} \hat{y}\right)
$$

Next, we discuss a specific reflector, with the diameter of $D_{r}=100 \lambda_{0}\left(f_{0}=100 \mathrm{GHz}\right)$ and the f-number of $f_{\#}^{r}=0.6$, illuminated by a unitary TM polarized plane wave with the skew angle of $\theta_{s}=2.3^{\circ}, \phi_{s}=0^{\circ}$. Fig. 4.15 shows the variation of linear and coma phase with respect to $\theta$. It can be seen that the linear phase varies linearly, while the coma phase is nonlinear and reaches maximum at the edge.

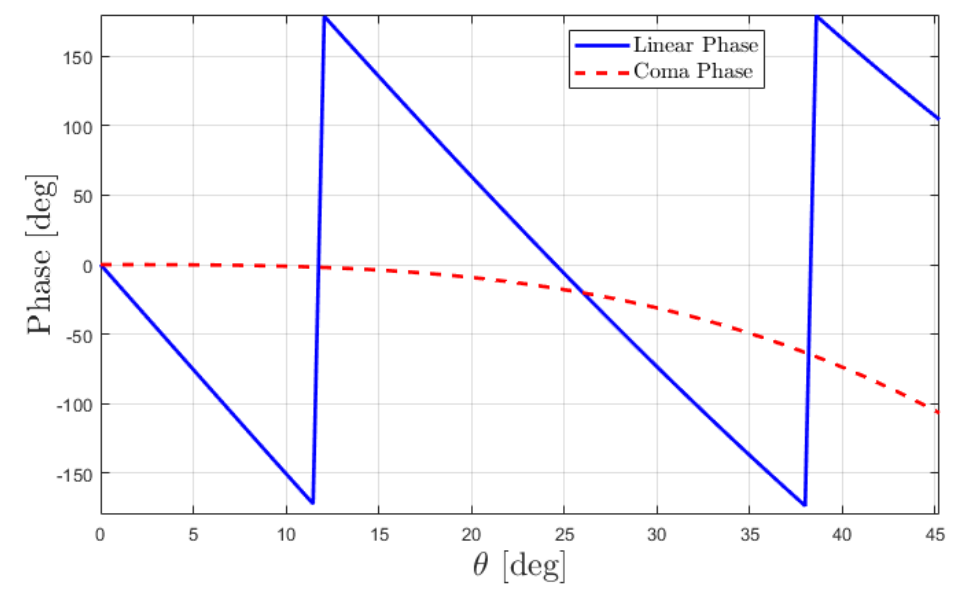

Figure 4.15: Variation of linear and coma phase for a parabolic reflector with $D_{r}=100 \lambda_{0}\left(f_{0}=100 \mathrm{GHz}\right)$ and $f_{\#}^{r}=0.6$, illuminated by a unitary TM polarized plane wave with the skew angle of $\theta_{s}=2.3^{\circ}, \phi_{s}=0^{\circ}$.

Furthermore, if we observe the field focalized on the focal plane by the same reflector, we can understand these phase terms more clearly. The reflector is illuminated by two plane waves: broadside and $\theta_{s}=2.3^{\circ}=4 \lambda_{0} / D_{r}, \phi_{s}=0^{\circ}$ (4 beams scanning). In this case, the flash point is calculated as: $\vec{\rho}_{f p}=4 \lambda_{0} f_{\#}^{r} \hat{x}$. As it can be seen in Fig. 4.16, the focalized fields are evaluated by resorting to the FO analysis with and without coma phase term, represented by the solid and dashed lines, respectively. Here we use the PO analysis as the reference that is marked with squares. 


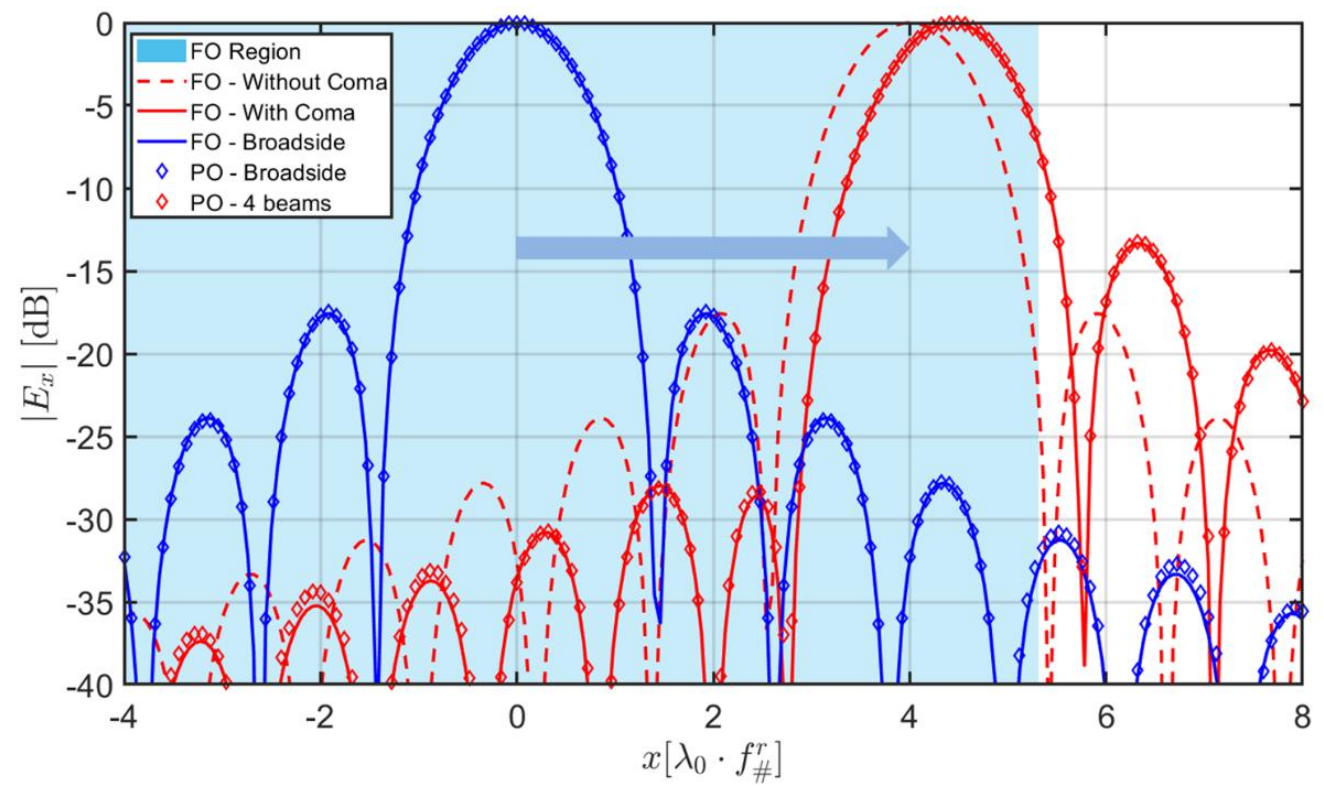

Figure 4.16: The $\mathrm{x}$-component of the electric fields on the focal plane of a parabolic reflector with $D_{r}=100 \lambda_{0}$ $\left(f_{0}=100 \mathrm{GHz}\right)$ and $f_{\#}^{r}=0.6$. The reflector is illuminated by two plane waves: broadside and $\theta_{s}=2.3^{\circ}, \phi_{s}=$ $0^{\circ}$. The solid lines are calculated by resorting to the FO analysis, while the squares represent the PO results. The dashed line is the case when the coma phase term is not included. One of the main planes $(y=0)$ is shown. The blue region is the FO applicability region.

It can be clearly seen in the figure that when the linear phase term is included only, the focalized field is a linear translation of the broadside one from the center to the 4-beam position, which is exact the flash point position. However, this translation is not accurate compared with the PO result. When the coma phase term is included, the result becomes accurate. The side lobes become asymmetric and the main beam is deviated from the flash point. This observation shows that the linear phase term corresponds to steer the maximum of the focal plane field of a QO component from the center of the focal plane to the flash point position. While the coma phase term leads to asymmetric side lobes and deviation of the main beam, which is quantified by the distance $\delta_{n}(\theta)$.

Practically, when we scan the incident field, it is useful to find the maximum skew angle where the coma phase term is sufficiently small and can be neglected. Within this angular region, the focalized field is almost a linear translation of the broadside one. We can set the phase error for neglecting the coma phase term as $\sigma$ :

$$
\vec{k}_{\rho} \cdot \vec{\rho}_{f p} \delta_{n}(\theta) \leq \sigma
$$

For a reflector, we have derived in Appendix I.1 the limit of the coma phase in terms of the number of beams, i.e. $N=\theta_{s} /(\lambda / D)$ :

$$
N_{\max }^{c o m a} \leq \frac{\sigma}{\pi}\left[2 f_{\#}+\sqrt{4 f_{\#}^{2}-1}\right]^{2}
$$


- Elliptical lens

In the case of an elliptical lens, $\delta_{n}(\theta)$ is derived as:

$$
\delta_{n}(\theta)=\frac{e\left(\cos \theta-\cos \theta_{0}\right)}{1-e \cos \theta}
$$

and the flash point is:

$$
\vec{\rho}_{f p}=R_{F O} \frac{\vec{k}_{\rho, s}}{k_{d}}=-R_{l} \frac{k_{0}}{k_{d}} \sin \theta_{s}\left(\cos \phi_{s} \hat{x}+\sin \phi_{s} \hat{y}\right)
$$

Moreover, the limit of the coma phase is derived in Appendix I.2:

$$
N_{\max }^{c o m a} \leq \frac{\sigma}{\pi} \frac{1-e \cos \theta_{\max }}{e \sin \theta_{\max }} \frac{1}{2 f_{\#} \cos \theta_{\max }-\sqrt{4 f_{\#}^{2}-1}}
$$

where $\theta_{\max }$ is the angle that maximizes the coma phase.

\section{- Hyperbolic lens}

In the case of a hyperbolic lens, $\delta_{n}(\theta)$ is:

$$
\delta_{n}(\theta)=\frac{e(\cos \theta-1)}{1-e \cos \theta}
$$

and the flash point is:

$$
\vec{\rho}_{f p}=R_{F O} \frac{\vec{k}_{\rho, s}}{k_{0}}=-f \frac{k_{d}}{k_{0}} \sin \theta_{s}\left(\cos \phi_{s} \hat{x}+\sin \phi_{s} \hat{y}\right)
$$

We can also derive the limit of the coma phase (Appendix I.3):

$$
N_{\text {max }}^{c o m a} \leq \frac{\sigma}{\pi e}\left[2 f_{\#}(e-1)\left(2 f_{\#}+\sqrt{4 f_{\#}^{2}-1}\right)-e\right]
$$

\subsubsection{Point source incidence}

In the case of an elliptical mirror, we use a point source to generate the incident field. We can place a source at either the upper focal plane or the lower focal plane.

\section{- Case 1: Source placed at the upper focal plane}

In Fig. 4.17, a source is placed at the upper focal plane with the displacement $\vec{\rho}_{s}$. In this case, the mirror and the FO sphere are parameterized by $\left(\theta_{2}, \phi_{2}\right)$, while the incident field is defined by $\left(\theta_{1}, \phi_{1}\right)$. 


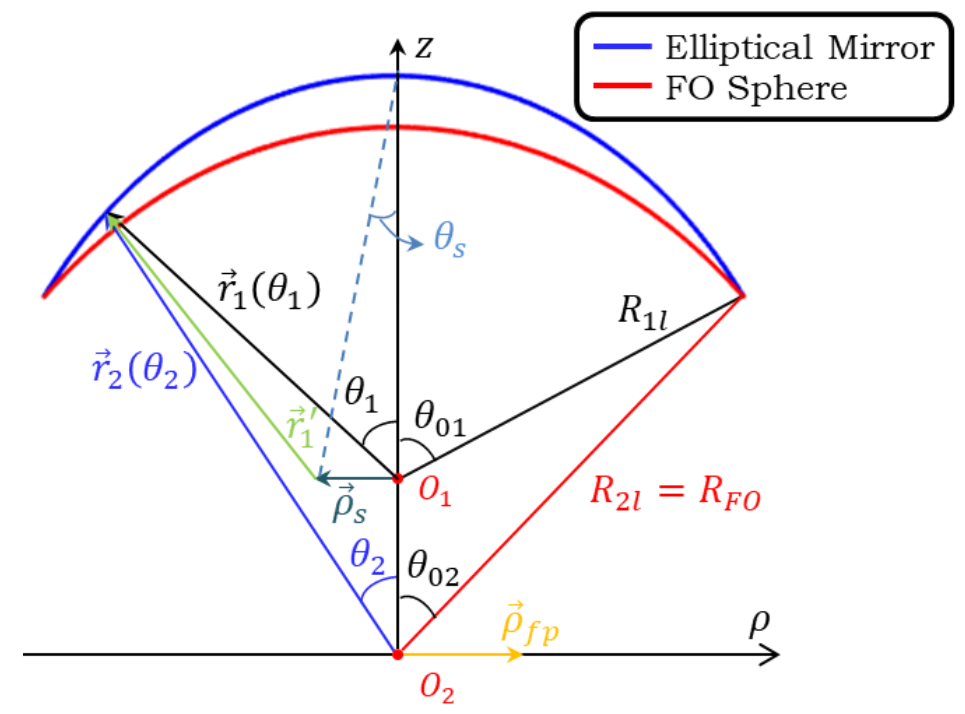

Figure 4.17: Geometry used to evaluate the progressive phase shift for an elliptical mirror with a point source placed at the upper focal plane.

Here we assume the approximation in Eq. (4.44) is still applicable, and define the progressive phase term as $\Phi=\Phi_{1}$. To calculate $\Phi_{1}$, we can express $\left|\vec{r}_{1}^{\prime}\right|$ as:

$$
\left|\vec{r}_{1}^{\prime}\right|=\left|\vec{r}_{1}-\vec{\rho}_{s}\right|=\sqrt{\left(\vec{r}_{1}-\vec{\rho}_{s}\right) \cdot\left(\vec{r}_{1}-\vec{\rho}_{s}\right)}=r_{1} \sqrt{1-\frac{2}{r_{1}}\left(\hat{r}_{1} \cdot \vec{\rho}_{s}\right)+\frac{\rho_{s}^{2}}{r_{1}^{2}}}
$$

where $r_{1}=\left|\vec{r}_{1}\right|, \hat{r}_{1}=\vec{r}_{1} / r_{1}$, and $\rho_{s}=\left|\vec{\rho}_{s}\right|$. Eq. (4.63) can be approximated by expanding the square root for small argument to the second order, i.e. $\sqrt{1+x} \simeq 1+\frac{x}{2}-\frac{x^{2}}{8}+\cdots$ :

$$
\left|\vec{r}_{1}^{\prime}\right|=r_{1}-\hat{r}_{1} \cdot \vec{\rho}_{s}+\frac{\rho_{s}^{2}}{2 r_{1}}\left[1-\left(\hat{r}_{1} \cdot \hat{\rho}_{s}\right)^{2}\right]+\frac{\rho_{s}^{3}}{2 r_{1}^{2}}\left(\hat{r}_{1} \cdot \hat{\rho}_{s}\right)
$$

where $\hat{\rho}_{s}=\vec{\rho}_{s} / \rho_{s}$. By using the value of $\left|\vec{r}_{1}^{\prime}\right|$, the incident field can be expressed as follows:

$$
\begin{gathered}
\vec{E}_{i}=\left(E_{i}^{\perp} \hat{\phi}+E_{i}^{\|} \hat{\theta}\right) \frac{e^{-j k_{0}\left|\vec{r}_{1}^{\prime}\right|}}{\left|\vec{r}_{1}^{\prime}\right|} \approx \frac{\left(E_{i}^{\perp} \hat{\phi}+E_{i}^{\|} \hat{\theta}\right)}{r_{1}} e^{-j k_{0}\left[r_{1}-\hat{r}_{1} \cdot \vec{\rho}_{S}+\frac{\rho_{S}^{2}}{2 r_{1}}\left[1-\left(\hat{r}_{1} \cdot \hat{\rho}_{S}\right)^{2}\right]+\frac{\rho_{S}^{3}}{2 r_{1}^{2}}\left(\hat{r}_{1} \cdot \hat{\rho}_{S}\right)\right]} \\
=\frac{\left(E_{i}^{\perp} \hat{\phi}+E_{i}^{\|} \hat{\theta}\right)}{r_{1}} e^{-j k_{0} r_{1}} e^{-j k_{0}\left[-\hat{r}_{1} \cdot \vec{\rho}_{S}+\frac{\rho_{S}^{2}}{2 r_{1}}\left[1-\left(\hat{r}_{1} \cdot \hat{\rho}_{S}\right)^{2}\right]+\frac{\rho_{S}^{3}}{2 r_{1}^{2}}\left(\hat{r}_{1} \cdot \hat{\rho}_{S}\right)\right]}
\end{gathered}
$$

Therefore, we can approximate $\vec{E}_{i}$ by the broadside illumination with a progressive phase term:

$$
\vec{E}_{i} \simeq \vec{E}_{i}\left(\vec{\rho}_{s}=0\right) \mathrm{e}^{-j \Phi_{1}}
$$

where $\Phi_{1}$ is expressed as below:

$$
\Phi_{1}=k_{0}\left[-\hat{r}_{1} \cdot \vec{\rho}_{s}+\frac{\rho_{s}^{2}}{2 r_{1}}\left[1-\left(\hat{r}_{1} \cdot \hat{\rho}_{s}\right)^{2}\right]+\frac{\rho_{s}^{3}}{2 r_{1}^{2}}\left(\hat{r}_{1} \cdot \hat{\rho}_{s}\right)\right]
$$

Next, we can divide the phase term $\mathrm{e}^{-j \Phi_{1}}$, similar to Eq. (4.52), into linear phase term and coma phase term: 


$$
e^{-j \Phi_{1}}=e^{j k_{0} \hat{r}_{1} \cdot \vec{\rho}_{s}} e^{-j k_{0} \frac{\rho_{S}^{2}}{2 r_{1}}\left[1-\left(\hat{r}_{1} \cdot \widehat{\rho}_{S}\right)^{2}\right]} e^{-j k_{0} \frac{\rho_{S}^{3}}{2 r_{1}^{2}}\left(\hat{r}_{1} \cdot \widehat{\rho}_{S}\right)}
$$

Since the mirror is parameterized by $\vec{r}_{2}\left(\theta_{2}\right)$, we can represent $\vec{r}_{1}\left(\theta_{1}\right)$ as a function of $\vec{r}_{2}\left(\theta_{2}\right)$, and calculate $\hat{r}_{1} \cdot \vec{\rho}_{s}$ as follows (Appendix H.1):

$$
\hat{r}_{1} \cdot \vec{\rho}_{s}=\frac{r_{2}}{r_{1}} \hat{r}_{2} \cdot \vec{\rho}_{s}=\frac{r_{2}}{r_{1}} \sin \theta_{2} \hat{\rho} \cdot \vec{\rho}_{s}
$$

By substituting Eq. (4.69) in Eq. (4.68), and defining a compensation phase term $\Phi_{\text {comp } 1}$ :

$$
e^{-j \Phi_{1}}=e^{j \frac{r_{2}}{r_{1}} \vec{k}_{\rho} \cdot \vec{\rho}_{s}} e^{-j \Phi_{c o m p 1}}
$$

where $\vec{k}_{\rho}=k_{0} \sin \theta_{2} \hat{\rho}$ and $\Phi_{\text {comp } 1}=k_{0}\left[\frac{\rho_{s}^{2}}{2 r_{1}}\left[1-\left(\hat{r}_{1} \cdot \hat{\rho}_{s}\right)^{2}\right]+\frac{\rho_{s}^{3}}{2 r_{1}^{2}}\left(\hat{r}_{1} \cdot \hat{\rho}_{s}\right)\right]$.

Moreover, $r_{2} / r_{1}$ can be expressed as follows (Appendix H.1):

$$
\frac{r_{2}}{r_{1}}=M_{1}\left(1+\Phi_{c o m a 1}\left(\theta_{2}\right)\right)
$$

where $M_{1}=R_{2 l} / R_{1 l}$, and $\Phi_{\text {coma1 }}\left(\theta_{2}\right)$ is:

$\Phi_{\text {coma } 1}\left(\theta_{2}\right)=-$

$$
\frac{e^{2}\left(\cos \theta_{1} \cos \theta_{02}-\cos \theta_{2} \cos \theta_{01}\right)+e\left(\cos \theta_{01}-\cos \theta_{1}+\cos \theta_{02}-\cos \theta_{2}\right)}{\left(1-e \cos \theta_{2}\right)\left(1+e \cos \theta_{01}\right)}
$$

If we define the flash point $\vec{\rho}_{f p}$ as:

$$
\vec{\rho}_{f p}=M_{1} \vec{\rho}_{s}
$$

By substituting Eq. (4.71) in Eq. (4.70), we can divide the progressive phase term into three terms:

$$
e^{-j \Phi_{1}}=e^{-j \Phi_{c o m p 1}} e^{j \vec{k}_{\rho} \cdot \vec{\rho}_{f p}} e^{j \vec{k}_{\rho} \cdot \vec{\rho}_{f p} \Phi_{c o m a 1}}
$$

The first term, $e^{-j \Phi_{c o m p} 1}$, is the compensation phase term, the second term, $e^{j \vec{k}_{\rho} \cdot \vec{\rho}_{f p}}$, is the linear phase term, and the last term, $e^{j \vec{k}_{\rho} \cdot \vec{\rho}_{f p} \Phi_{c o m a 1}}$, is the coma phase term. Consequently, as described in Eq. (4.45), the analytical GO field can be approximated as:

$$
\vec{E}_{G O}\left(\vec{\rho}_{S}\right) \approx \vec{E}_{G O}\left(\vec{\rho}_{s}=0\right) e^{-j \Phi_{c o m p 1}} e^{j \vec{k}_{\rho} \cdot \vec{\rho}_{f p}} e^{j \vec{k}_{\rho} \cdot \vec{\rho}_{f p} \Phi_{c o m a 1}}
$$

Furthermore, $\Phi_{\text {comp } 1}$ can be approximated by a constant within a specific region $\left|\rho_{s}\right| \leq \rho_{s, \text { max }}^{f}$ (Appendix H.2):

$$
\Phi_{\mathrm{comp} 1} \approx k_{0} \frac{\rho_{s}^{2}}{2 R_{1 l}}
$$

If the displacement of the source is inside this region, the GO field can be expressed as:

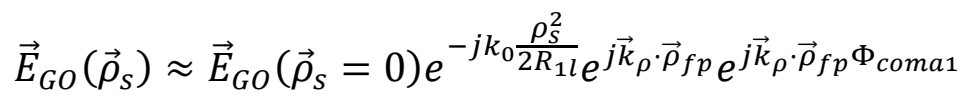




\section{- Case 2: Source placed at lower focal plane}

In Fig. 4.18, a source is placed at the lower focal plane with the displacement $\vec{\rho}_{S}$. In this case, the mirror and the FO sphere are parameterized by $\left(\theta_{1}, \phi_{1}\right)$, while the incident field is defined by $\left(\theta_{2}, \phi_{2}\right)$.

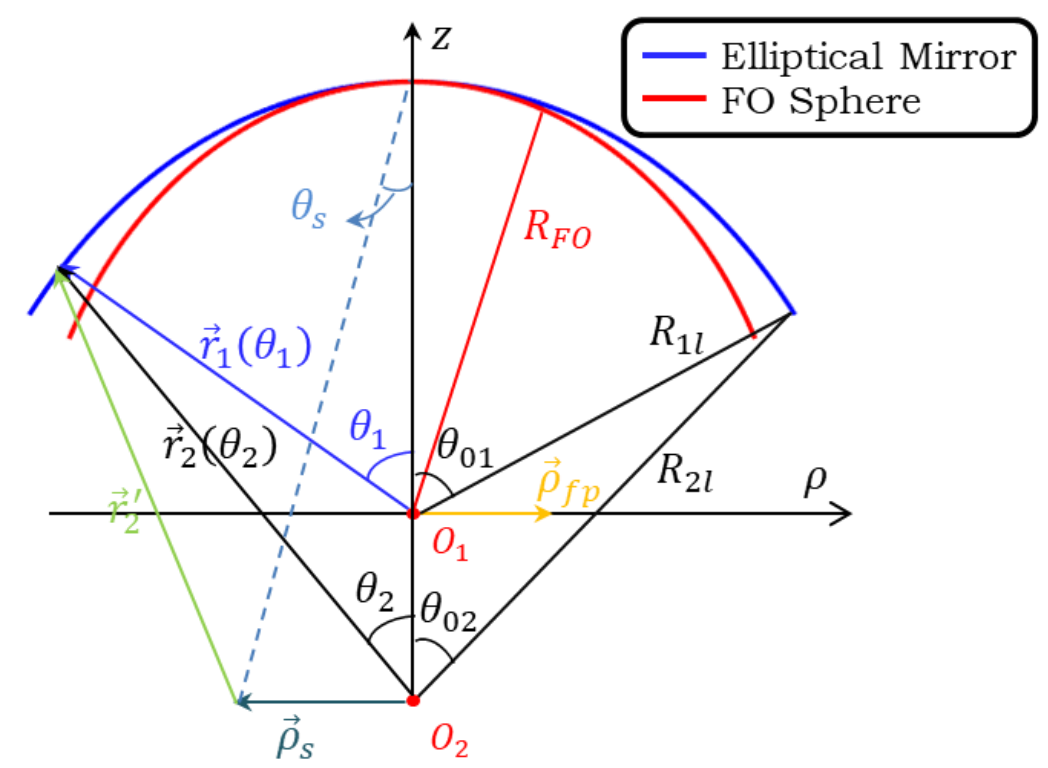

Figure 4.18: Geometry used to evaluate the progressive phase shift for an elliptical mirror with a point source placed at the lower focal plane.

We define the progressive phase term as $\Phi=\Phi_{2}$. Similar to the steps in case 1 , we can expand $\left|\vec{r}_{2}^{\prime}\right|$ and obtain $\Phi_{2}$ :

$$
\Phi_{2}=k_{0}\left[-\hat{r}_{2} \cdot \vec{\rho}_{s}+\frac{\rho_{s}^{2}}{2 r_{2}}\left[1-\left(\hat{r}_{2} \cdot \hat{\rho}_{s}\right)^{2}\right]+\frac{\rho_{s}^{3}}{2 r_{2}^{2}}\left(\hat{r}_{2} \cdot \hat{\rho}_{s}\right)\right]
$$

After similar derivations described in case 1, one can divide the progressive phase term into compensation phase term, linear phase term and coma phase term:

$$
e^{-j \Phi_{2}}=e^{-j k_{0} \Phi_{c o m p} 2} e^{j \vec{k}_{\rho} \cdot \vec{\rho}_{f p}} e^{j \vec{k}_{\rho} \cdot \vec{\rho}_{f p} \Phi_{c o m a 2}}
$$

where $\Phi_{\text {comp } 2}=k_{0}\left[\frac{\rho_{s}^{2}}{2 r_{2}}\left[1-\left(\hat{r}_{2} \cdot \hat{\rho}_{s}\right)^{2}\right]+\frac{\rho_{s}^{3}}{2 r_{2}^{2}}\left(\hat{r}_{2} \cdot \hat{\rho}_{s}\right)\right]$ and $\vec{k}_{\rho}=k_{0} \sin \theta_{1} \hat{\rho}$. The flash point is:

$$
\vec{\rho}_{f p}=M_{2} \vec{\rho}_{s}, M_{2}=\frac{R_{1 l}}{R_{2 l}}
$$

and $\Phi_{\text {coma2 }}\left(\theta_{1}\right)$ is:

$$
\begin{aligned}
& \Phi_{\text {coma } 2}\left(\theta_{1}\right)= \\
& \frac{e^{2}\left(\cos \theta_{1} \cos \theta_{02}-\cos \theta_{2} \cos \theta_{01}\right)+e\left(\cos \theta_{01}-\cos \theta_{1}+\cos \theta_{02}-\cos \theta_{2}\right)}{\left(1+e \cos \theta_{1}\right)\left(1-e \cos \theta_{02}\right)}
\end{aligned}
$$


Therefore, as described in Eq. (4.45), the analytical GO field can be approximated as:

$$
\vec{E}_{G O}\left(\vec{\rho}_{S}\right) \approx \vec{E}_{G O}\left(\vec{\rho}_{S}=0\right) e^{-j k_{0} \Phi_{c o m p} 2} e^{j \vec{k}_{\rho} \cdot \vec{\rho}_{f p}} e^{j \vec{k}_{\rho} \cdot \vec{\rho}_{f p} \Phi_{c o m a 2}}
$$

Moreover, $\Phi_{\text {comp } 2}$ can be approximated by a constant within a specific region $\left|\rho_{s}\right| \leq \rho_{s, \text { max }}^{f}$ (Appendix H.2):

$$
\Phi_{\text {comp2 }} \approx \frac{\rho_{s}^{2}}{2 R_{2 l}}
$$

If the displacement of the source is inside this region, the GO field can be expressed as:

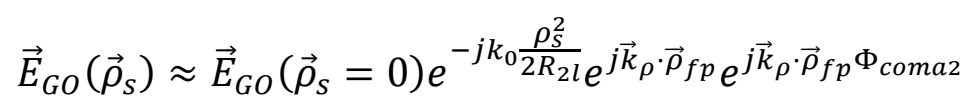

\section{- Illustration of phase terms}

Here we show an example to better illustrate the linear, coma, and compensation phase terms. We consider an elliptical mirror with the diameter of $D_{m}=100 \lambda_{0}\left(f_{0}=300 \mathrm{GHz}\right)$, the semimajor axis of $a=86 \lambda_{0}$, and the focal distance of $c=17 \lambda_{0}$, i.e. the eccentricity is $e=0.2$. The mirror is illuminated by a unitary Huygens source placed at the upper focal plane, with the electric current oriented along $\hat{x}$ and a displacement in $\mathrm{x}$-direction, $x_{s}=3.3 \lambda_{0} f_{\#}^{m}$. In this case, the $\mathrm{f}$-number is calculated as $f_{\#}^{m}=1$.

Fig. 4.19 shows the variation of linear, coma, and compensation phase with respect to $\theta$. As it can be seen, the linear phase varies linearly, the coma phase has a maximum around $\theta \approx$ $\frac{1}{2} \theta_{02}=15^{\circ}$, and the compensation phase increases slowly and behaves like a constant. In this case, the region where the compensation phase can be approximated as a constant is calculated as $\rho_{s, \text { max }}^{f}=3 \lambda_{0} f_{\#}^{m}$. The source displacement is $\rho_{s}=3.3 \lambda_{0} f_{\#}^{m}$; therefore, this approximation is not applicable.

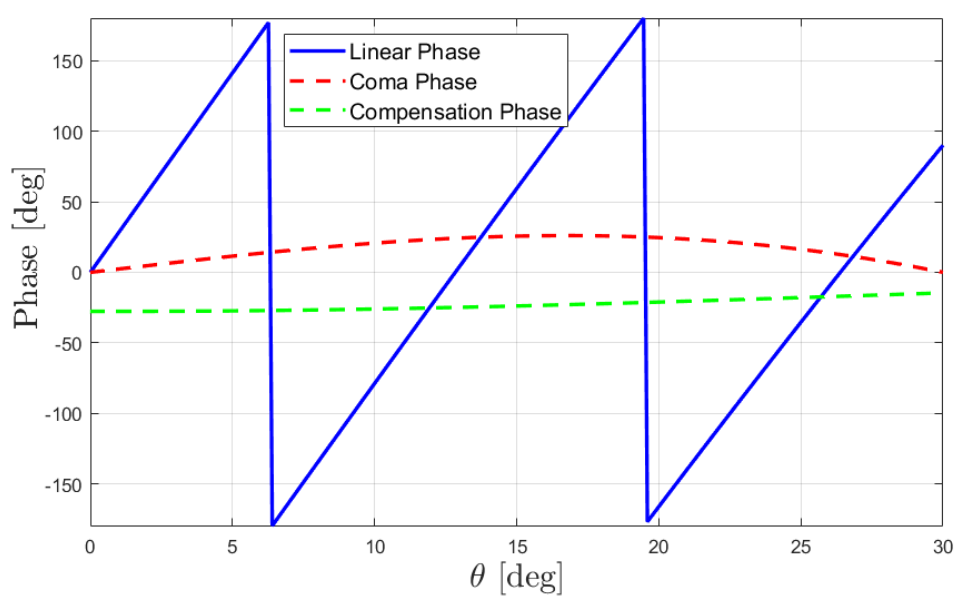

Figure 4.19: Variation of linear, coma, and compensation phase for an elliptical mirror with $D_{m}=100 \lambda_{0}\left(f_{0}=\right.$ $300 \mathrm{GHz}), f_{\#}^{m}=1$, and $e=0.2$, illuminated by a unitary Huygens source placed at the upper focal plane, with the electric current oriented along $\hat{x}$ and a displacement in $\mathrm{x}$-direction, $x_{s}=3.3 \lambda_{0} f_{\#}^{m}$. 
Furthermore, we can observe the field focalized on the focal plane by the same mirror and the same source, as shown in Fig. 4.20. As it can be seen, the fields evaluated by using the FO analysis are compared with the PO result. Moreover, in FO analysis, we compare the field calculated by using all phase terms with the one with only the linear phase term and the one without the compensation phase term. Here the case of broadside incidence is also shown as a contrast.

By using Eq. (4.73), one can calculate the flash point as: $\vec{\rho}_{f p}=-4.5 \lambda_{0} f_{\#}^{m} \hat{x}$. Therefore, the linear phase term translates the main beam to this flash point, shown by the dashed red line in Fig. 4.20a. When the coma phase term is included, the main beam deviates from the flash point and the side lobes become asymmetric, represented by the dashed black line. However, when the compensation phase term is included, the amplitude remains almost the same, shown by the red solid line. Therefore, we turn to the phase plot (Fig. 4.20b). It can be seen when the compensation phase term is neglected, the phase of the focal plane field changes obviously within the FO applicability region. Therefore, this phase term must be included to compensate the phase of the focal plane field. It is worth mentioning that the compensation phase is quantified by the source displacement, $\vec{\rho}_{s}$. When $\vec{\rho}_{s}$ is very small, the compensation phase can be approximated as a constant, or even be neglected.

\section{- Limit of the coma phase}

For the limit of the coma phase, we introduced the term $\Phi_{\text {coma }}$ instead of $\delta_{n}$. Therefore, the condition in Eq. (4.55) should be modified:

$$
\vec{k}_{\rho} \cdot \vec{\rho}_{f p} \Phi_{\text {coma }} \leq \sigma
$$

It is derived in Appendix I.4 that when the observation plane is at the lower focal plane, the maximum number of beams is:

$$
N_{\text {max }}^{\text {coma }} \leq \frac{\sigma}{2 \pi f_{\#}} \frac{1}{\max \left(\sin \theta_{2} \Phi_{\text {coma } 1}\left(\theta_{2}\right)\right)}
$$

where the term " $\max \left(\sin \theta_{2} \Phi_{\text {coma } 1}\left(\theta_{2}\right)\right)$ " can be roughly approximated by substituting $\theta_{2}=$ $\frac{1}{2} \theta_{02}$; or accurately evaluated by using numerical calculation. When the observation plane is at the upper focal plane:

$$
N_{\text {max }}^{\text {coma }} \leq \frac{\sigma}{2 \pi f_{\#}} \frac{1}{\max \left(\sin \theta_{1} \Phi_{\text {coma2 }}\left(\theta_{1}\right)\right)}
$$

where the term " $\max \left(\sin \theta_{1} \Phi_{\text {coma2 }}\left(\theta_{1}\right)\right)$ " can be roughly approximated by substituting $\theta_{1}=$ $\frac{1}{2} \theta_{01}$, or calculated numerically. 


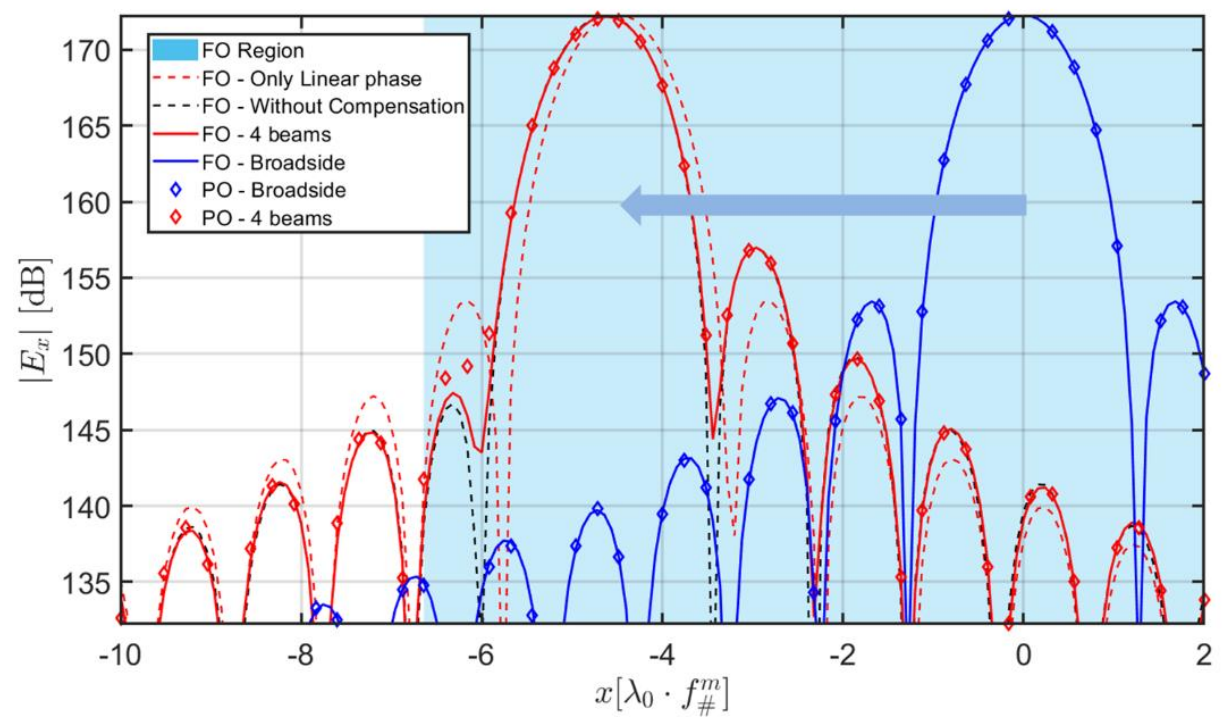

(a)

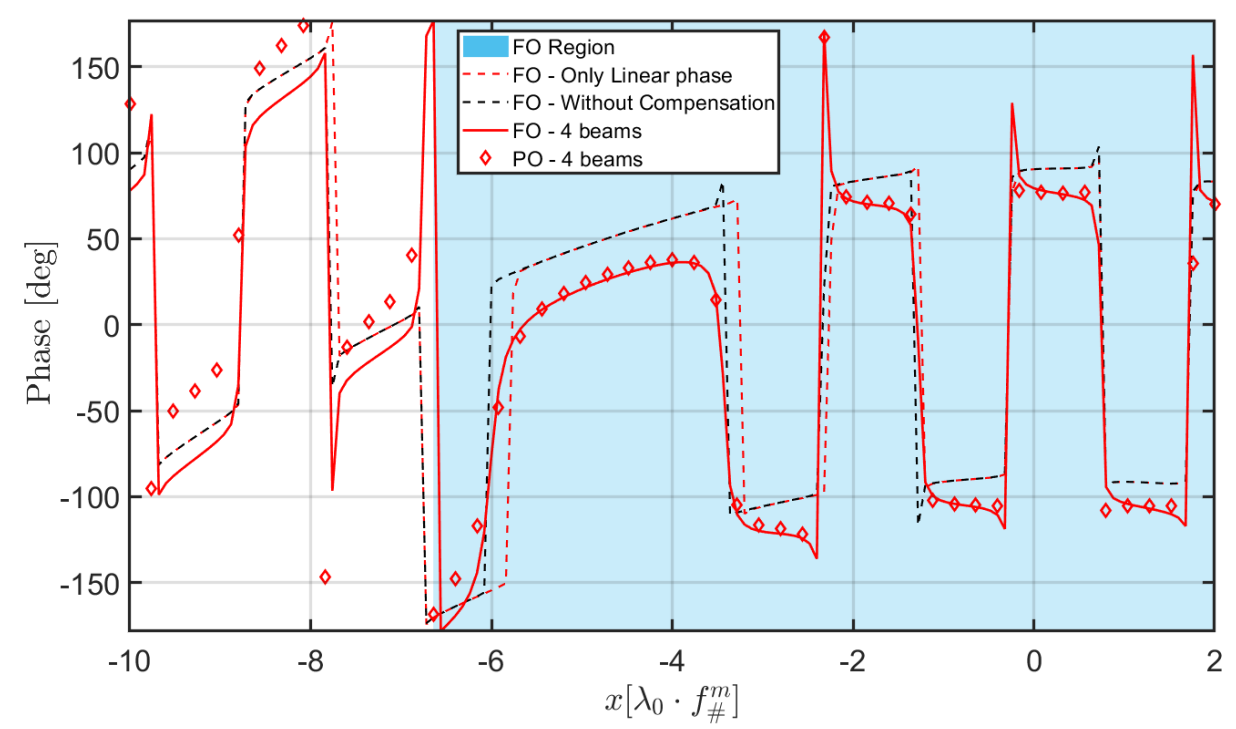

(b)

Figure 4.20: The $\mathrm{x}$-component of the electric fields on the focal plane of an elliptical mirror with $D_{m}=$ $100 \lambda_{0}\left(f_{0}=300 \mathrm{GHz}\right), f_{\#}^{m}=1$ and the eccentricity $e=0.2$. The mirror is illuminated by a unitary Huygens sources placed at the upper focal plane, with the electric current oriented along $\hat{x}$ and a displacement in $\mathrm{x}$ direction, $x_{s}=3.3 \lambda_{0} f_{\#}^{m}$. The solid lines are calculated by resorting to the FO analysis, while the squares represent the PO results. The dashed red and black lines represent the cases when the coma and compensation phase terms are neglected, respectively: (a) Amplitude. (b) Phase. One of the main planes $(y=0)$ is shown. The blue region is the FO applicability region. 


\subsubsection{Comparison of coma limits}

We have derived the limits of the coma phase (coma limits) for parabolic reflectors, elliptical lenses, hyperbolic lenses and elliptical mirrors. It can be found that a coma limit is always a

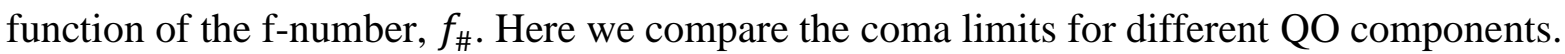

Here we choose the operative frequency as $f_{0}=300 \mathrm{GHz}$ and fix the diameter as $D=$ $100 \lambda_{0}$. This diameter is not suitable for elliptical lenses; therefore, we compare parabolic reflectors, hyperbolic lenses and elliptical mirrors. For hyperbolic lenses, the relative permittivity is chosen as $\varepsilon_{r}=2$. In the case of elliptical mirrors, the eccentricity is selected as $e=0.2$.

The comparison of the coma limits is shown in Fig. 4.21. As it can be seen, all coma limits $\left(N_{\max }^{\operatorname{coma}}\right)$ increase monotonically with respect to the f-number, $f_{\#}$. For a fixed $f_{\#}$, the elliptical mirror with the observation at the upper focal plane always has the highest limit; while the hyperbolic lens always has the lowest one. Therefore, we can conclude that elliptical mirrors are less sensitive to the coma phase term compared with parabolic reflectors and hyperbolic lenses.

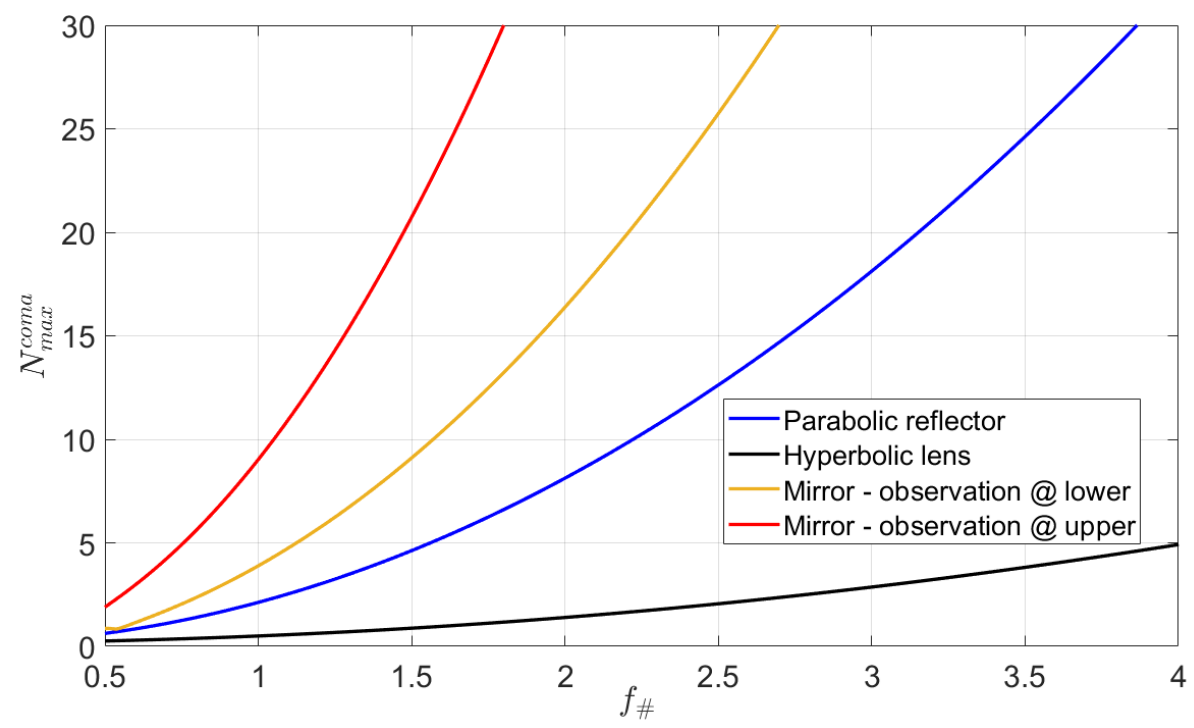

Figure 4.21: Comparison of coma limits for parabolic reflectors, hyperbolic lenses, and elliptical mirrors (with observation at upper and lower focal planes). 


\section{Chapter 5: Validation of GO fields and PWS}

In chapter 3, we have discussed the importance of PWS and shown that it is proportional to the GO fields on a FO sphere. And later in chapter 4, GO ray fields and analytical GO fields were derived explicitly. Finally, in this chapter, we focus on validating GO fields and their corresponding PWS.

The radius of a FO sphere is chosen as large as possible since the applicability region for the FO approximations is proportional to this radius, as described in Eq. (3.12). As a result, the distance between the surface of a QO component and the FO sphere could be very small. In such a case, the fields on the FO sphere are numerically difficult to be evaluated by using the PO analysis, namely it is hard to validate GO fields directly on a FO sphere. In FO analysis, it is known in Eq. (3.9) that focal plane fields can be obtained by integrating GO fields. Consequently, instead of validating the fields on the FO sphere, one can validate the fields on the focal plane which can be calculated easily and accurately by the PO analysis. If focal plane fields are validated by the PO, one can assume GO fields are also validated. This assumption is also applicable for validating PWS. One can validate the focal plane fields evaluated by using the coherent FO method. If the focal plane fields are validated, the PWS is also assumed to be validated.

In section 5.1, GO fields are validated. Focal plane fields calculated by using the FO analysis are compared with the ones evaluated by using the PO analysis. In section 5.2, first we show some numerical examples of PWS. We compare the full CFO spectrum with the linearized CFO spectrum. And then we validate the coherent FO for all QO components.

\subsection{Validating the derived GO fields}

In this section, we validate both GO ray fields (section 4.1) and analytical GO fields (section 4.2) by validating the focal plane fields with the PO analysis. Here we use Eq. (3.9) to calculate focal plane fields, i.e. use both electric and magnetic GO fields. We call the focal plane fields calculated by using the GO ray fields "GO-FO", and the ones evaluated by using the analytical GO fields “Analytical FO”.

Three types of QO components, namely elliptical lenses, hyperbolic lenses, and elliptical mirrors, with TM polarized incidence are discussed here. Additional examples of the other QO components and TE validation are listed in Appendix J. Here we validate both amplitude and phase and show one of the main planes $(y=0)$. FO applicability regions are highlighted by blue regions, and phase is wrapped from $-180^{\circ}$ to $180^{\circ}$. 


\subsubsection{Elliptical lens}

An elliptical silicon $\left(\varepsilon_{r}=11.9\right)$ lens with $D_{l}=5 \lambda_{0}\left(f_{0}=300 \mathrm{GHz}\right)$ and $f_{\#}^{l}=0.6$ is introduced here. A quarter-wavelength matching layer at $300 \mathrm{GHz}$ made of Parylene $\left(\varepsilon_{m}=\right.$ 2.62 ) is applied. For such a lens, two different scenarios are analyzed: One is the case with small skew angle; the other is the case with large skew angle.

\section{- Case 1: Small skew angle}

In this case, the lens is illuminated by a unitary TM polarized plane wave with the skew angle of $\theta_{S}=10^{\circ}, \phi_{S}=0^{\circ}$, meaning analytical GO expressions are applicable. Fig. 5.1 shows the $\mathrm{x}$-component of the electric fields on the focal plane of the lens. The focal plane fields evaluated by resorting to the analytical FO and the GO-FO approaches are compared with the one obtained using the PO. Here the 3D PO field is plotted in the inset of the figure. It can be seen in the figure, the agreement is within the accepted error margin inside the FO applicability region, for both the amplitude and phase.

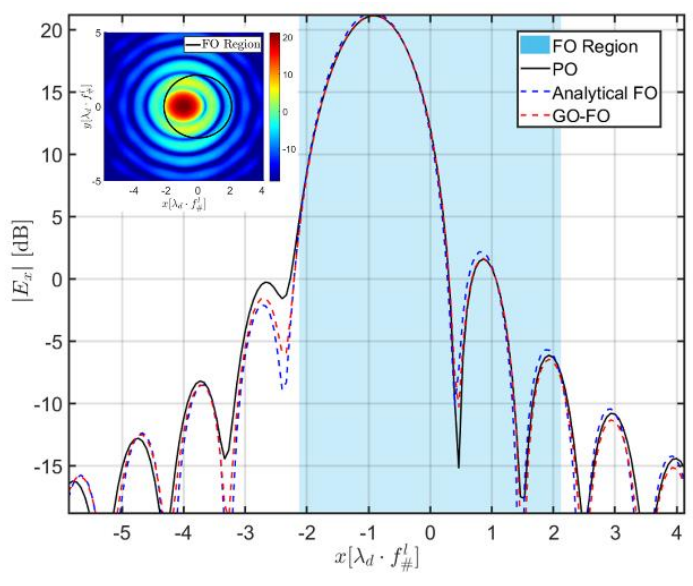

(a)

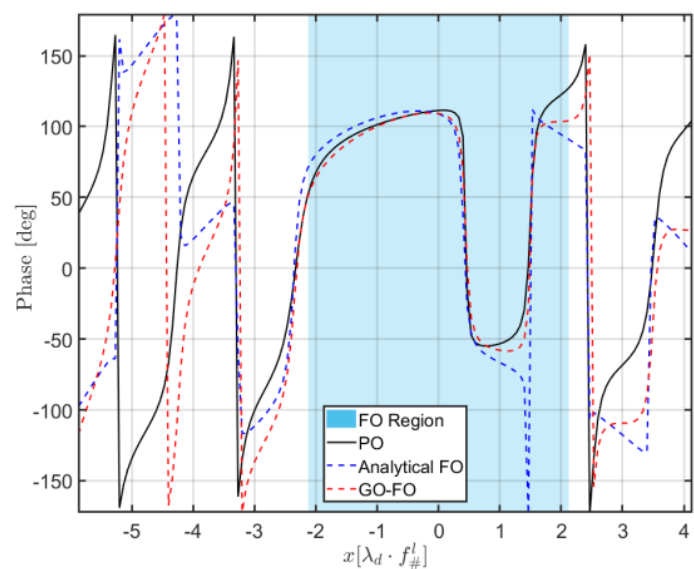

(b)

Figure 5.1: The $x$-components of the electric fields on the focal plane of an elliptical silicon $\left(\varepsilon_{r}=11.9\right)$ lens with $D_{l}=5 \lambda_{0}\left(f_{0}=300 \mathrm{GHz}\right)$ and $f_{\#}^{l}=0.6$, illuminated by a unitary TM polarized plane wave with the skew angle of $\theta_{s}=10^{\circ}, \phi_{s}=0^{\circ}$. Here a quarter-wavelength matching layer at $300 \mathrm{GHz}$ made of Parylene $\left(\varepsilon_{m}=2.62\right)$ is applied. The focal plane fields calculated by using the analytical FO and the GO-FO approaches are compared with the one obtained using the PO: (a) Amplitude. (b) Phase. One of the main planes $(y=0)$ is shown. Blue region is the FO applicability region. Inset is the 3D PO field.

\section{- Case 2: Large skew angle}

In this case, the lens is illuminated by a unitary TM polarized plane wave with the skew angle of $\theta_{s}=20^{\circ}, \phi_{s}=0^{\circ}$, meaning analytical GO expressions are no longer accurate. Fig. 5.2 shows the $\mathrm{x}$-component of the electric fields on the focal plane of the lens. The focal plane 
fields evaluated by using the analytical FO and the GO-FO approaches are compared with the one obtained using the PO. As it can be seen, the analytical FO approach does not match with the PO. While for the GO-FO approach, the agreement is very good within the FO applicability region. Therefore, when the skew angle is larger than $11^{\circ}$, one should always apply the GOFO approach to ensure accuracy.

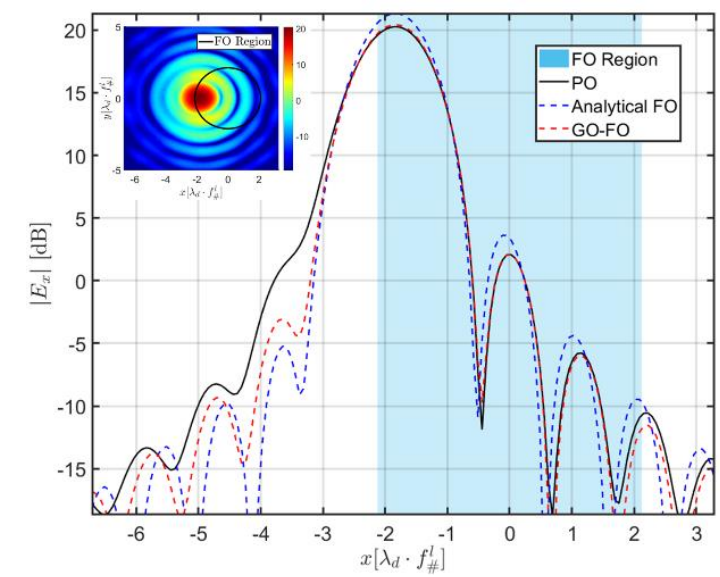

(a)

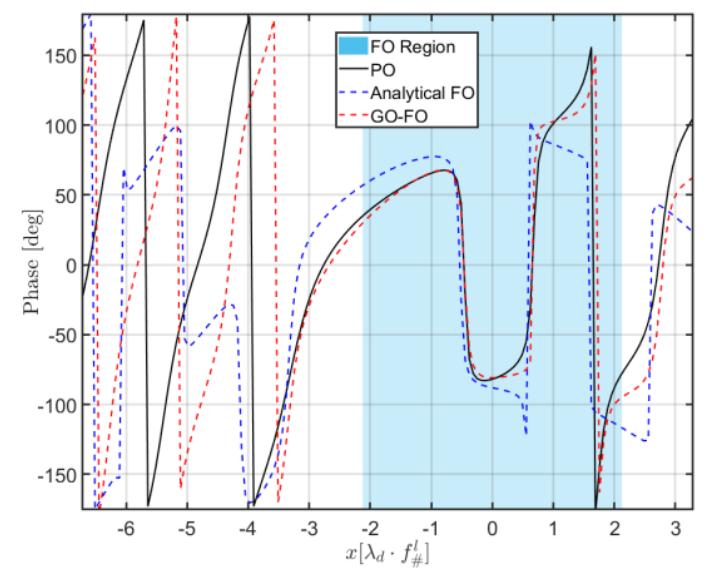

(b)

Figure 5.2: The x-components of the electric fields on the focal plane of an elliptical silicon $\left(\varepsilon_{r}=11.9\right)$ lens with $D_{l}=5 \lambda_{0}\left(f_{0}=300 \mathrm{GHz}\right)$ and $f_{\#}^{l}=0.6$, illuminated by a unitary TM polarized plane wave with the skew angle of $\theta_{s}=20^{\circ}, \phi_{s}=0^{\circ}$. Here a quarter-wavelength matching layer at $300 \mathrm{GHz}$ made of Parylene $\left(\varepsilon_{m}=2.62\right)$ is applied. The focal plane fields calculated by using the analytical FO and the GO-FO approaches are compared with the one obtained using the PO: (a) Amplitude. (b) Phase. One of the main planes $(y=0)$ is shown. Blue region is the FO applicability region. Inset is the 3D PO field.

\subsubsection{Hyperbolic lens}

Fig. 5.3 shows the $\mathrm{x}$-component of the electric fields on the focal plane of a hyperbolic plastic $\left(\varepsilon_{r}=2\right)$ lens with $D_{h}=100 \lambda_{0}\left(f_{0}=300 \mathrm{GHz}\right)$ and $f_{\#}^{h}=1$. The incident field is a unitary TM polarized plane wave with the skew angle of $\theta_{s}=3.2\left(\lambda_{d} / D_{h}\right)=1.3^{\circ}, \phi_{s}=0^{\circ}$. The focal plane fields evaluated by using the analytical FO and the GO-FO approaches are compared with the one obtained using the PO. Here the 3D PO field is plotted in the inset of the figure.

As it can be seen in the figure, for the amplitude comparison, the analytical FO and the GO-FO approaches are validated by the PO within the FO applicability region. For the phase comparison, the analytical FO approach is still very good while the GO-FO approach performs some acceptable error. This is due to the fact that the interpolation operation involved in calculating the GO ray fields for a hyperbolic lens is not very accurate for a small f-number geometry. 


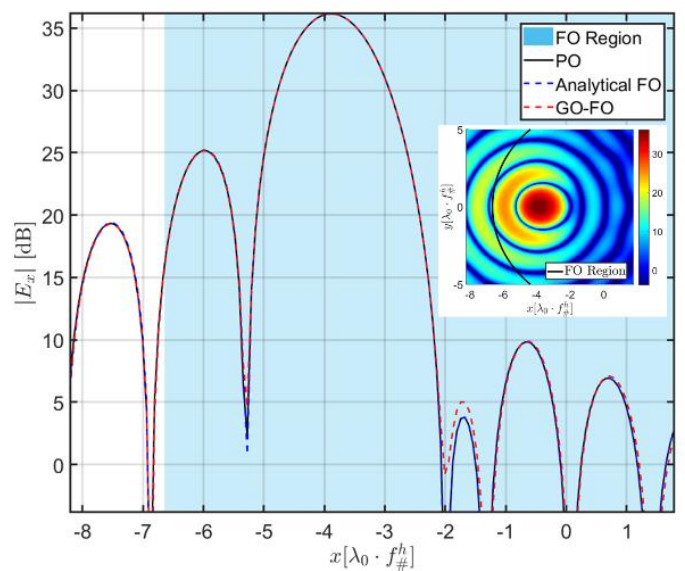

(a)

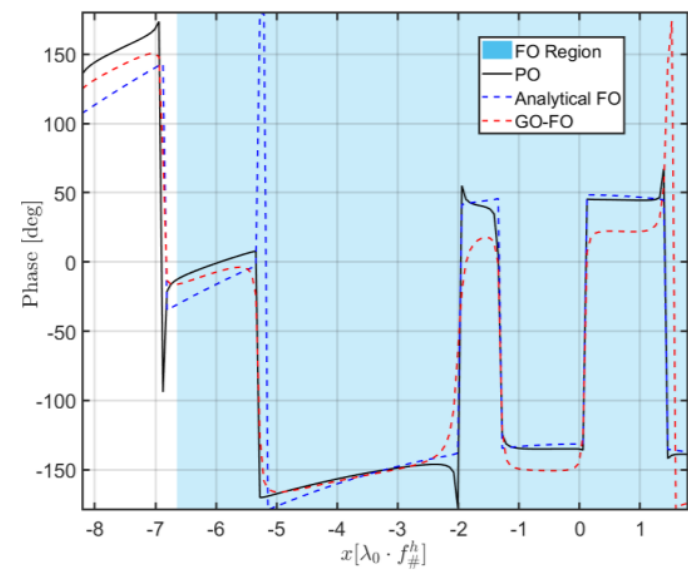

(b)

Figure 5.3: The $\mathrm{x}$-component of the electric fields on the focal plane of a hyperbolic plastic $\left(\varepsilon_{r}=2\right)$ lens with $D_{h}=100 \lambda_{0}\left(f_{0}=300 \mathrm{GHz}\right)$ and $f_{\#}^{h}=1$, illuminated by a unitary TM polarized plane wave with the skew angle of $\theta_{s}=3.2\left(\lambda_{d} / D_{h}\right)=1.3^{\circ}, \phi_{s}=0^{\circ}$. The focal plane fields calculated by using the analytical FO and the GO-FO approaches are compared with the one obtained using the PO: (a) Amplitude. (b) Phase. One of the main planes $(y=0)$ is shown. Blue region is the FO applicability region. Inset is the 3D PO field.

\subsubsection{Elliptical mirror}

In the case of an elliptical mirror, a source can be placed at either its upper focal plane or its lower focal plane. Here we consider a mirror with the diameter of $D_{m}=100 \lambda_{0}\left(f_{0}=\right.$ $300 \mathrm{GHz}$ ), the semi-major axis of $a=80 \lambda_{0}$, and the focal distance of $c=20 \lambda_{0}$, i.e. the eccentricity is $e=0.25$.

\section{- Case 1: Source placed at the upper focal plane}

In this case, the f-number is calculated as $f_{\#}^{m}=0.95$. The incident field is generated by a unitary Huygens source placed at the upper focal plane, with the electric current oriented along $\hat{x}$ and a displacement in $\mathrm{x}$-direction, $x_{s}=2.2 \lambda_{0} f_{\#}^{m}$. Fig. 5.4 shows the $\mathrm{x}$-component of the electric fields on the focal plane of the mirror. The focal plane fields calculated by using the analytical FO and the GO-FO approaches are compared with the one obtained using the PO. It can be seen in the figure, the analytical FO and the GO-FO approaches are in fair agreement with the $\mathrm{PO}$ inside the FO applicability region, for both the amplitude and the phase. 


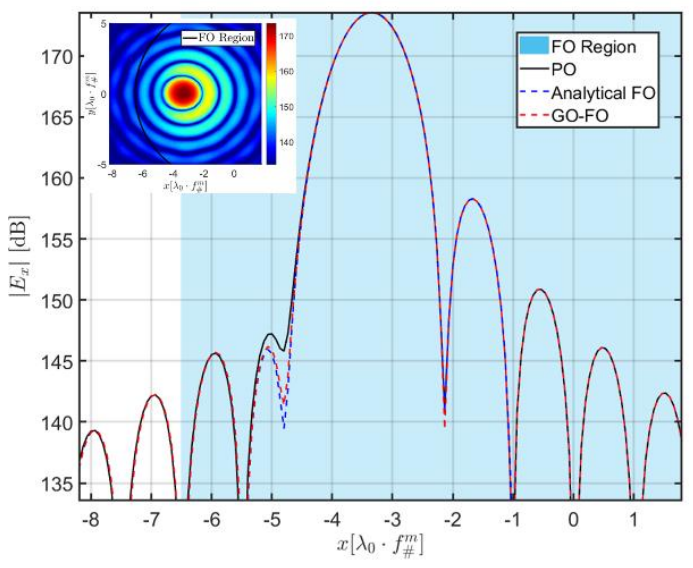

(a)

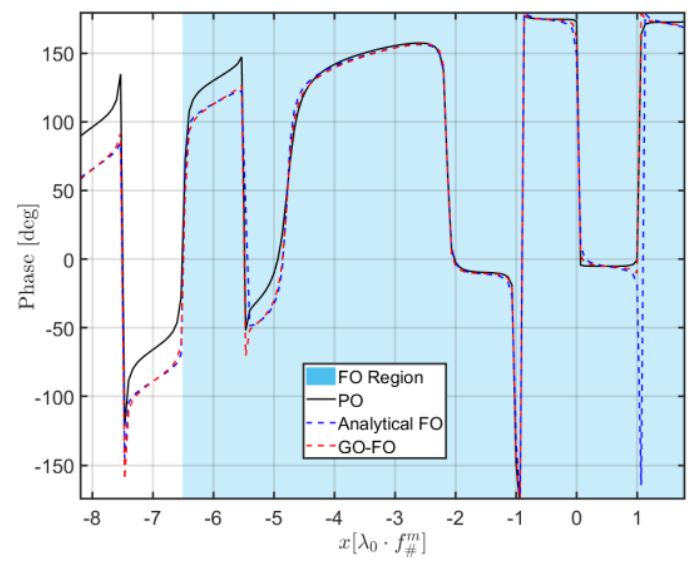

(b)

Figure 5.4: The x-component of the electric fields on the focal plane of an elliptical mirror with $D_{m}=$ $100 \lambda_{0}\left(f_{0}=300 \mathrm{GHz}\right), f_{\#}^{m}=0.95$ and $e=0.25$. The mirror is illuminated by a unitary Huygens source placed at the upper focal plane, with the electric current oriented along $\hat{x}$ and a displacement in $\mathrm{x}$-direction, $x_{s}=$ $2.2 \lambda_{0} f_{\#}^{m}$. The focal plane fields evaluated by using the analytical FO and the GO-FO approaches are compared with the one obtained using PO: (a) Amplitude. (b) Phase. One of the main planes $(y=0)$ is shown. Blue region is the FO applicability region. Inset is the $3 \mathrm{D}$ PO field.

\section{- Case 2: Source placed at the lower focal plane}

In this case, the f-number is calculated as $f_{\#}^{m}=0.6$. The unitary Huygens source is placed at the lower focal plane, with the electric current oriented along $\hat{x}$ and a displacement in $\mathrm{x}-$ direction, $x_{s}=4.7 \lambda_{0} f_{\#}^{m}$. Fig. 5.5 shows the x-component of the electric fields on the focal plane of the mirror. The focal plane fields evaluated by resorting to the analytical FO and the GO-FO approaches are compared with the one obtained using the PO. As it can be seen in the figure, the agreement is very good inside the FO applicability region, for both the amplitude and the phase.

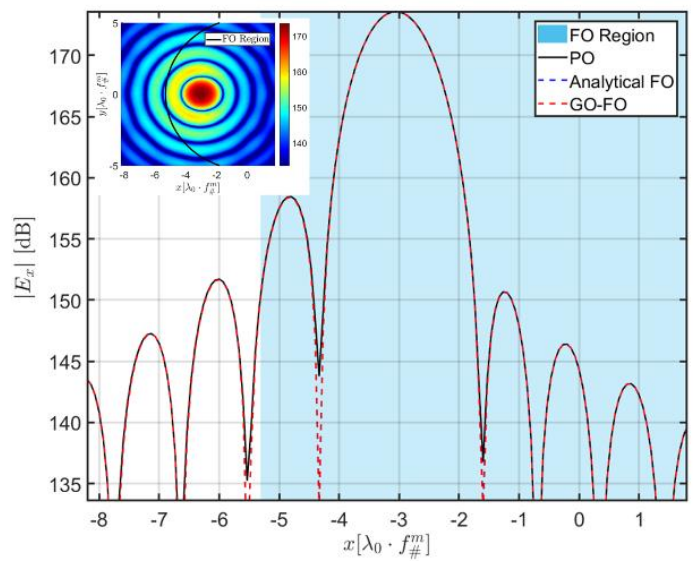

(a)

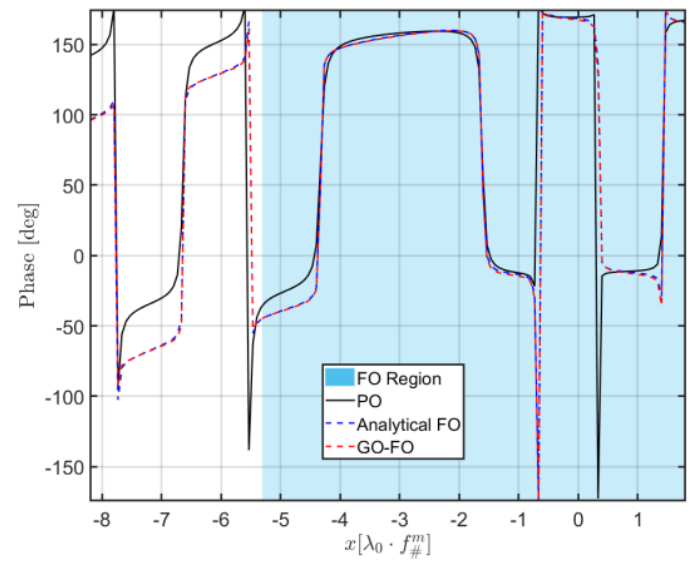

(b)

Figure 5.5: The $\mathrm{x}$-component of the electric fields on the focal plane of an elliptical mirror with $D_{m}=$ $100 \lambda_{0}\left(f_{0}=300 \mathrm{GHz}\right), f_{\#}^{m}=0.6$ and $e=0.25$. The mirror is illuminated by a unitary Huygens source placed at the lower focal plane, with the electric current oriented along $\hat{x}$ and a displacement in $\mathrm{x}$-direction, $x_{s}=$ $4.7 \lambda_{0} f_{\#}^{m}$. The focal plane fields evaluated by using the analytical FO and the GO-FO approaches are compared with the one obtained using PO: (a) Amplitude. (b) Phase. One of the main planes $(y=0)$ is shown. Blue region is the FO applicability region. Inset is the 3D PO field. 


\subsection{Numerical examples and validation of the coherent FO}

It has been discussed in section 3.3 that the coherent FO can evaluate the PWS of the focal plane field that includes also its phase property. Two types of spectra are derived. The first one is the full CFO spectrum that is evaluated by calculating a convolution integral. The second one is the linearized CFO spectrum. By using the linearization approximation on the quadratic phase term, the convolution is approximated by a linear shift in the spectral domain, and the full spectrum becomes a linearized local spectrum.

In section 5.2.1, numerical examples of the preceding spectra are shown. In section 5.2.2, the coherent FO method is validated for all QO components. The focal plane fields evaluated by using the coherent FO are compared with the ones calculated by the FO with quadratic phase term, i.e. the analytical FO and GO-FO discussed in section 5.1.

\subsubsection{Numerical examples of PWS}

It has been discussed in section 3.3 that when the observation point is not far away from the center of the focal plane, i.e. the quadratic phase term is negligible, the PWS of the focal plane field is $\vec{E}_{F O}\left(k_{x}, k_{y}\right)$ which is proportional to the GO fields. However, when we observe a point further from the center, the quadratic phase term must be included and the PWS cannot be approximated by $\vec{E}_{F O}\left(k_{x}, k_{y}\right)$. In such a case, it is derived in the coherent FO method that the full CFO spectrum of the focal plane field is a convolution between two spectral functions:

$$
\vec{E}_{f}\left(-k_{x},-k_{y}\right)=F\left(-k_{x},-k_{y}\right) * \vec{E}_{F O}\left(k_{x}, k_{y}\right)
$$

where $F\left(k_{x}, k_{y}\right)$ is the FT of the quadratic phase term and $\vec{E}_{F O}\left(-k_{x},-k_{y}\right)$ is described in Eq. (3.26). Moreover, the convolution operation in Eq. (5.1) can be simplified by applying the linearization approximation on the quadratic phase term. The linearized CFO spectrum can be expressed as follows:

$$
\vec{E}_{f}\left(-k_{x},-k_{y}\right)=e^{-j k \frac{\left|\vec{\rho}_{o}\right|^{2}}{2 R_{F O}}} \vec{E}_{F O}\left(\vec{k}_{\rho}+\vec{k}_{o}\right) e^{j\left(\vec{k}_{\rho}+\vec{k}_{o}\right) \cdot \vec{\rho}_{o}}
$$

where $\vec{\rho}_{o}$ is the position where we introduce the linearization, $\vec{k}_{\rho}=k_{x} \hat{x}+k_{y} \hat{y}$, and $\vec{k}_{o}=$ $\frac{k}{R_{F O}} \vec{\rho}_{o}$.

To better illustrate these spectra, we take a parabolic reflector as an example. We first assume the quadratic phase term can be neglected; therefore, the spectrum is $\vec{E}_{F O}\left(k_{x}, k_{y}\right)$. Next, we calculate the convolution integral in Eq. (5.1) to evaluate the full CFO spectrum. Furthermore, we apply the linearization approximation and calculate the linearized CFO 
spectrum described in Eq. (5.2). Finally, the focal plane fields evaluated by using $\vec{E}_{F O}\left(k_{x}, k_{y}\right)$, the full spectrum and the linearized spectrum are compared with the one calculated by the FO with quadratic phase term, i.e. the analytical FO and GO-FO described in section 5.1.

The parabolic reflector has the diameter of $D_{r}=500 \lambda_{0}\left(f_{0}=300 \mathrm{GHz}\right)$ and the f-number of $f_{\#}^{r}=4$, illuminated by a unitary TM polarized plane wave with the skew angle of $\theta_{s}=$ $20\left(\lambda_{0} / D_{r}\right)=2.3^{\circ}, \phi_{s}=0^{\circ}$.

\section{- Spectrum of $\vec{E}_{F O}\left(k_{x}, k_{y}\right)$}

When the quadratic phase term is neglected, the PWS of the focal plane field is $\vec{E}_{F O}\left(k_{x}, k_{y}\right)$, as shown in Fig. 5.6. Since the rim angle of the reflector is $\theta_{0}$, the spectrum is bounded within $k_{\rho 0}=k_{0} \sin \theta_{0}$. Moreover, the amplitude spectrum is divided into $\left|\vec{E}_{F O, \theta}\right|$ and $\left|\vec{E}_{F O, \phi}\right|$ components.

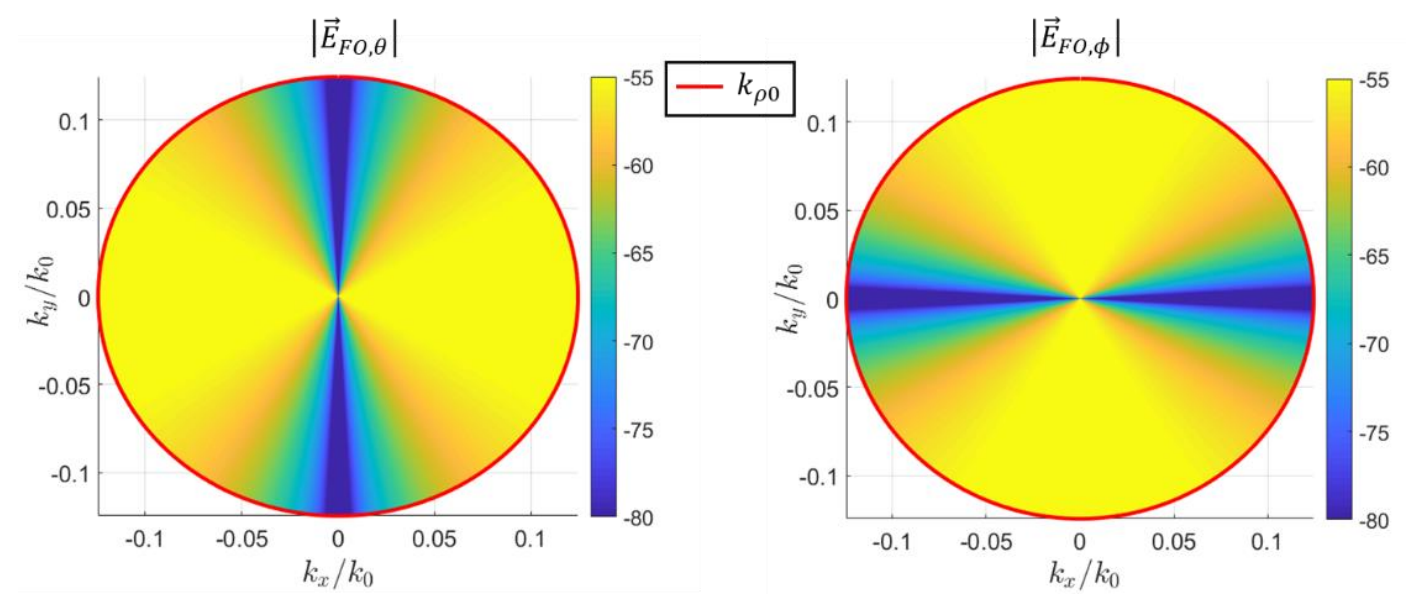

Figure 5.6: Amplitude of the spectral function $\vec{E}_{F O}\left(k_{x}, k_{y}\right)$ of a parabolic reflector with $D_{r}=500 \lambda_{0}\left(f_{0}=\right.$ $300 \mathrm{GHz}$ ) and $f_{\#}^{r}=4$, illuminated by a unitary TM polarized plane wave with the skew angle of $\theta_{s}=$ $20\left(\lambda_{0} / D_{r}\right)=2.3^{\circ}, \phi_{s}=0^{\circ}$. The spectrum is bounded within $k_{\rho 0}$ : Left-hand side is the $\left|\vec{E}_{F O, \theta}\right|$ component while the right-hand side is the $\left|\vec{E}_{F O, \phi}\right|$ component.

\section{- Full CFO spectrum}

Next, we consider convolving $\vec{E}_{F O}\left(-k_{x},-k_{y}\right)$ with $F\left(k_{x}, k_{y}\right)$ and calculating the full CFO spectrum, $\vec{E}_{f}\left(-k_{x},-k_{y}\right)$. The amplitude spectrum is shown in Fig. 5.7 and is divided into $\left|\vec{E}_{f, \theta}\right|$ and $\left|\vec{E}_{f, \phi}\right|$ components. Due to the property of the convolution operation, the spectrum is not bounded within $k_{\rho 0}$. As it can be seen, we limit the spectrum within $1.7 k_{\rho 0}$, since the spectrum outside this region is negligible. 

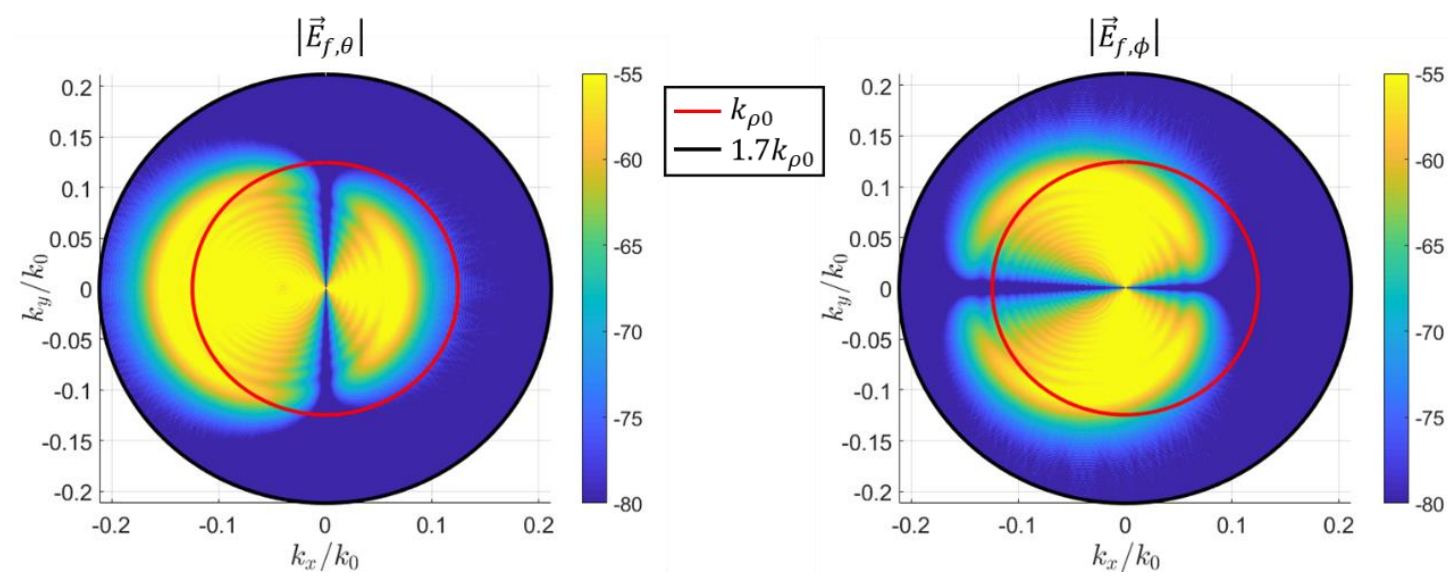

Figure 5.7: Amplitude of the full spectrum $\vec{E}_{f}\left(-k_{x},-k_{y}\right)$ of a parabolic reflector with $D_{r}=500 \lambda_{0}\left(f_{0}=\right.$ $300 \mathrm{GHz}$ ) and $f_{\#}^{r}=4$, illuminated by a unitary TM polarized plane wave with the skew angle of $\theta_{s}=$ $20\left(\lambda_{0} / D_{r}\right)=2.3^{\circ}, \phi_{s}=0^{\circ}$. The spectrum is bounded within $1.7 k_{\rho 0}$ : Left-hand side is the $\left|\vec{E}_{f, \theta}\right|$ component while the right-hand side is the $\left|\vec{E}_{f, \phi}\right|$ component.

\section{- Linearized CFO spectrum}

In this case, we apply the linearization approximation on the quadratic phase term, and linearize the full spectrum in the surrounding of the flash point position. The skew angle of the incident plane wave is $\theta_{s}=20\left(\lambda_{0} / D_{r}\right)=2.3^{\circ}, \phi_{s}=0^{\circ}$. Therefore, the linearization point is equal to the flash point and can be calculated as $\vec{\rho}_{o}=\vec{\rho}_{f p} \approx 20 \lambda_{0} f_{\#}^{r} \hat{x}$. Fig. 5.8 shows the amplitude of the linearized CFO spectrum, i.e. $\left|\vec{E}_{f}\left(-k_{x},-k_{y}\right)\right|=\left|\vec{E}_{F O}\left(\vec{k}_{\rho}+\vec{k}_{o}\right)\right|$, with $\left|\vec{E}_{f, \theta}\right|$ and $\left|\vec{E}_{f, \phi}\right|$ components. It can be seen in the figure that the linearized spectrum is a linear translation of $\left|\vec{E}_{F O}\left(k_{x}, k_{y}\right)\right|$ (Fig. 5.6), with the spectral shift $\vec{k}_{x o}=\frac{k_{0}}{R_{F O}} \vec{\rho}_{o}$.

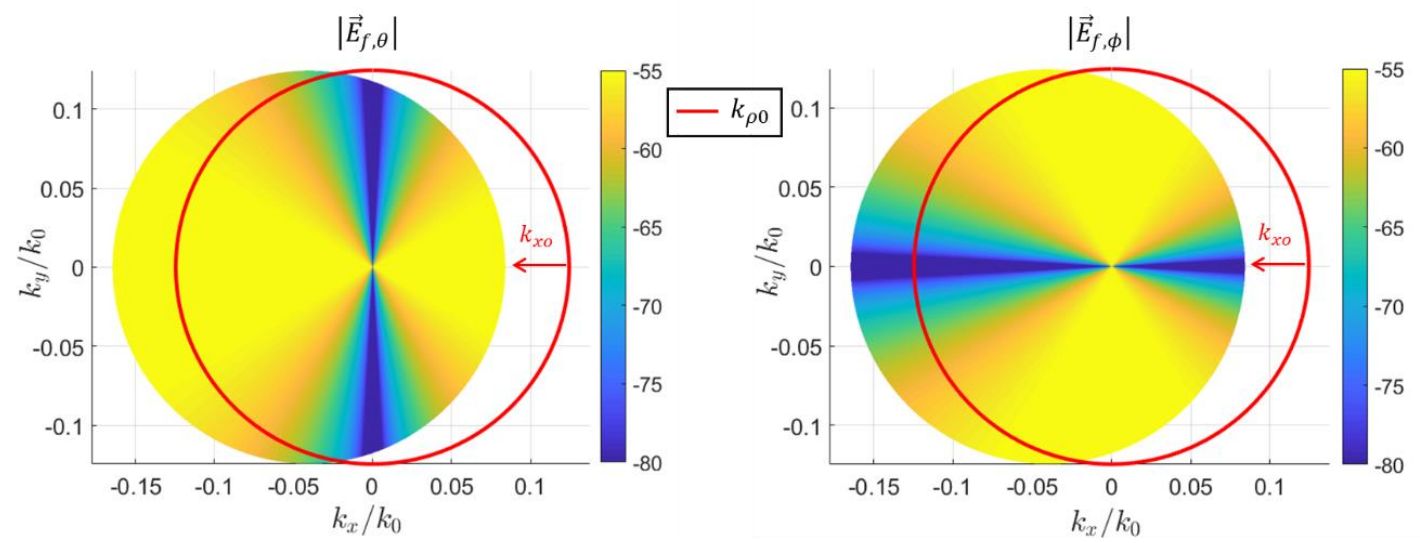

Figure 5.8: Amplitude of the linearized spectrum $\vec{E}_{f}\left(-k_{x},-k_{y}\right)$ of a parabolic reflector with $D_{r}=500 \lambda_{0}\left(f_{0}=\right.$ $300 \mathrm{GHz}$ ) and $f_{\#}^{r}=4$, illuminated by a unitary TM polarized plane wave with the skew angle of $\theta_{s}=$ $20\left(\lambda_{0} / D_{r}\right)=2.3^{\circ}, \phi_{s}=0^{\circ}$. The spectrum is shifted with $k_{x o}$ from the center and bounded within $k_{\rho 0}$ : Left-hand side is the $\left|\vec{E}_{f, \theta}\right|$ component while the right-hand side is the $\left|\vec{E}_{f, \phi}\right|$ component. 


\section{- Focal plane fields}

The focal plane field can be evaluated by integrating its PWS, as described in Eq. (3.33). Fig. 5.9 shows the $\mathrm{x}$-component of the electric fields on the focal plane of the parabolic reflector. The focal plane fields evaluated by using the full spectrum and the linearized spectrum are compared with the ones obtained using the FO with and without the quadratic phase term. Here for the linearized spectrum, the linearization point is chosen the same the flash point, i.e. $\vec{\rho}_{o} \approx 20 \lambda_{0} f_{\#}^{r} \hat{x}$.

It can be seen in the figure that the full-spectrum coherent FO is in fair agreement with the analytical FO within the FO applicability region. For the linearized-spectrum coherent FO, it is validated within the PWS applicability region. Outside this region, the phase error becomes larger. Here the PWS applicability region is the region where the linearization approximation is applicable. While for the FO without the quadratic phase term, the amplitude is accurate but the phase shows a large error. Therefore, the quadratic phase term must be included when the observation point is far away from the focus.

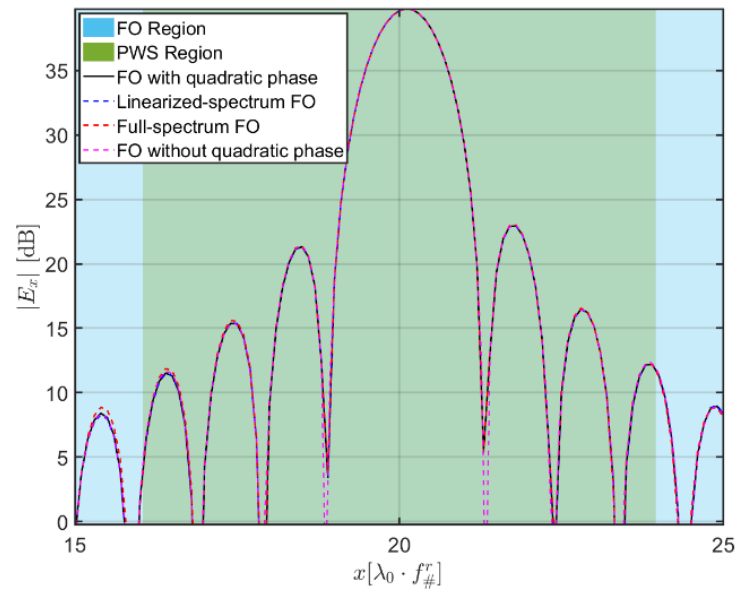

(a)

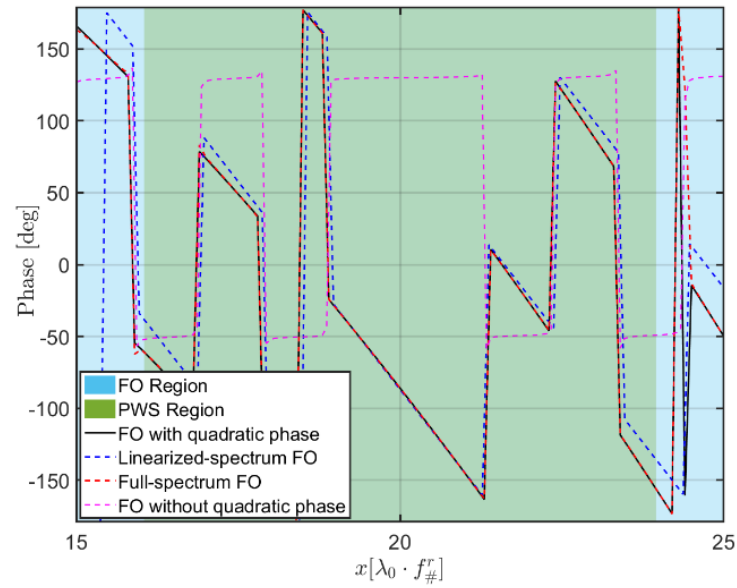

(b)

Figure 5.9: The $\mathrm{x}$-component of the electric fields on the focal plane of a parabolic reflector with $D_{r}=500 \lambda_{0}$ $\left(f_{0}=300 \mathrm{GHz}\right)$ and $f_{\#}^{r}=4$, illuminated by a unitary TM polarized plane wave with the skew angle of $\theta_{s}=$ $20\left(\lambda_{0} / D_{r}\right)=2.3^{\circ}, \phi_{s}=0^{\circ}$. The linearization point is chosen the same the flash point, i.e. $\vec{\rho}_{o} \approx 20 \lambda_{0} f_{\#}^{r} \hat{x}$. And the focal plane fields calculated by using the full-spectrum and linearized-spectrum coherent FO are compared with the ones obtained using the FO with and without quadratic phase term: (a) Amplitude. (b) Phase. One of the main planes $(y=0)$ is shown. Blue region is the FO applicability region and the green region is the PWS applicability region.

\subsubsection{Validation examples of the coherent FO}

The full-spectrum coherent FO is accurate within the entire FO applicability region; however, the calculation of the convolution integral requires large number of points to converge, which is time-consuming and numerically cumbersome. Moreover, full spectrum is not always needed in practice. One only needs to obtain a spectrum that is locally accurate around the position of 
a feed antenna. Therefore, in this subsection, we only validate the linearized-spectrum coherent FO, by validating the focal plane fields calculated by it.

Here the spectral function $\vec{E}_{F O}\left(k_{x}, k_{y}\right)$ is evaluated by using both electric and magnetic GO fields as:

$$
\vec{E}_{F O}\left(k_{x}, k_{y}\right)=\frac{j \pi R_{F O} e^{-j k R_{F O}}}{k_{z}}\left\{\left[\vec{E}_{G O}-\left(\hat{n} \cdot \vec{E}_{G O}\right) \hat{n}\right]-\zeta\left(\hat{n} \times \vec{H}_{G O}\right)\right\} \operatorname{circ}\left(k_{\rho}, k_{\rho 0}\right)
$$

Moreover, only TM validation is introduced here. Examples of TE validation are listed in Appendix K.

\section{- Elliptical lens}

Here an elliptical lens with a matching layer is considered. Fig. 5.10 shows the X-component of the electric fields on the focal plane of an elliptical silicon $\left(\varepsilon_{r}=11.9\right)$ lens with $D_{l}=5 \lambda_{0}$ $\left(f_{0}=300 \mathrm{GHz}\right)$ and $f_{\#}^{l}=0.6$. A quarter-wavelength matching layer at $300 \mathrm{GHz}$ made of Parylene $\left(\varepsilon_{m}=2.62\right)$ is applied. The lens is illuminated by a unitary TM polarized plane wave with the skew angle of $\theta_{s}=20^{\circ}, \phi_{s}=0^{\circ}$ which corresponds to a flash point $\vec{\rho}_{f p} \approx$ $-1.7 \lambda_{d} f_{\#}^{l} \hat{x}$. The linearization position is chosen the as the flash point, i.e. $\vec{\rho}_{o}=\vec{\rho}_{f p}$. And the focal plane field evaluated by using the coherent FO is compared with the one obtained using the GO-FO. As it can be seen, the coherent FO is in very good agreement with the GO-FO, for both the amplitude and the phase, within the PWS applicability region.

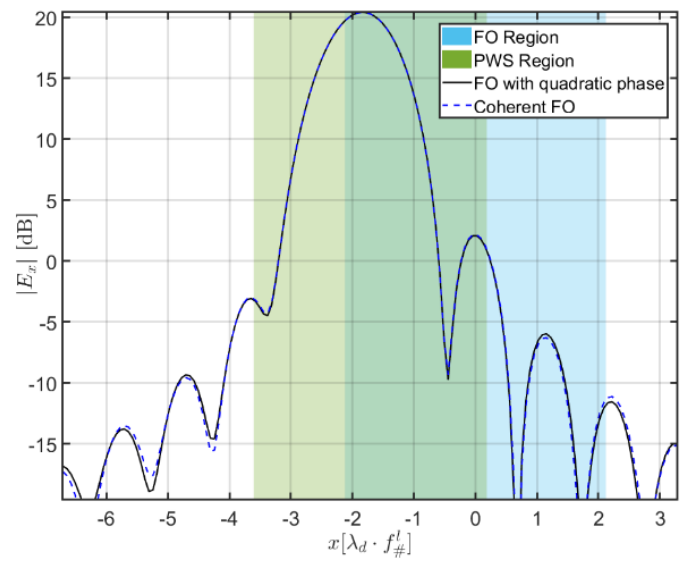

(a)

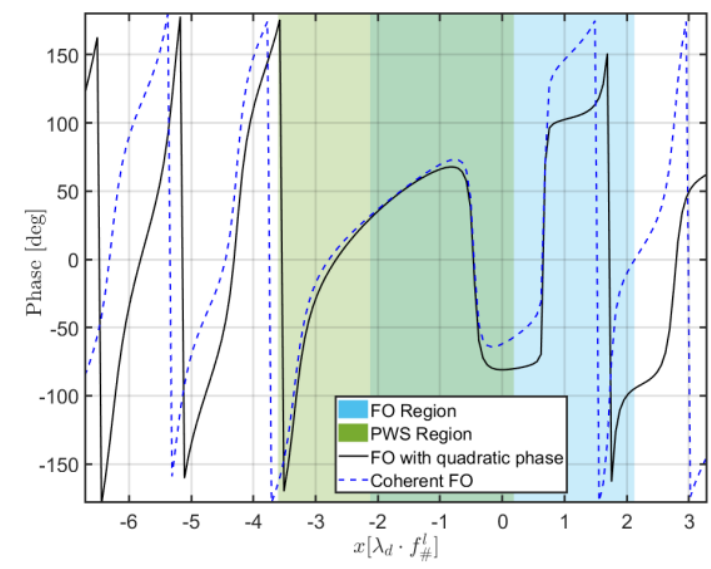

(b)

Figure 5.10: The x-component of the electric fields on the focal plane of an elliptical silicon $\left(\varepsilon_{r}=11.9\right)$ lens with $D_{l}=5 \lambda_{0}\left(f_{0}=300 \mathrm{GHz}\right)$ and $f_{\#}^{l}=0.6$, illuminated by a unitary TM polarized plane wave with the skew angle of $\theta_{s}=20^{\circ}, \phi_{s}=0^{\circ}$. Here a quarter-wavelength matching layer at $300 \mathrm{GHz}$ made of Parylene $\left(\varepsilon_{m}=2.62\right)$ is applied. The linearization point is chosen the same the flash point, i.e. $\vec{\rho}_{o} \approx-1.7 \lambda_{d} f_{\#}^{l} \hat{x}$. And the focal plane field calculated by using the coherent FO is compared with the one obtained using the GO-FO: (a) Amplitude. (b) Phase. One of the main planes $(y=0)$ is shown. Blue region is the FO applicability region and green region is the PWS applicability region. 
- Hemispherical lens

A hemispherical silicon $\left(\varepsilon_{r}=11.9\right)$ lens with $D_{h l}=5 \lambda_{0}\left(f_{0}=300 \mathrm{GHz}\right), f_{\#}^{h l}=0.6$, $R_{s p h}=2.6 \lambda_{0}$, and $L=0.362 R_{s p h}$ is introduced here. A quarter-wavelength matching layer at $300 \mathrm{GHz}$ made of Parylene $\left(\varepsilon_{m}=2.62\right)$ is applied. The lens is illuminated by a unitary TM polarized plane wave with the skew angle of $\theta_{s}=15^{\circ}, \phi_{s}=0^{\circ}$ which corresponds to a flash point $\vec{\rho}_{f p} \approx-1.3 \lambda_{d} f_{\#}^{h l} \hat{x}$. The linearization point is chosen the as the flash point. Fig. 5.11 shows the $\mathrm{x}$-component of the electric fields on the focal plane of the lens. The focal plane field evaluated by using the coherent FO is compared with the one obtained using the GO-FO. As it can be seen in the figure, the coherent FO is validated inside the PWS applicability region, for both the amplitude and the phase.

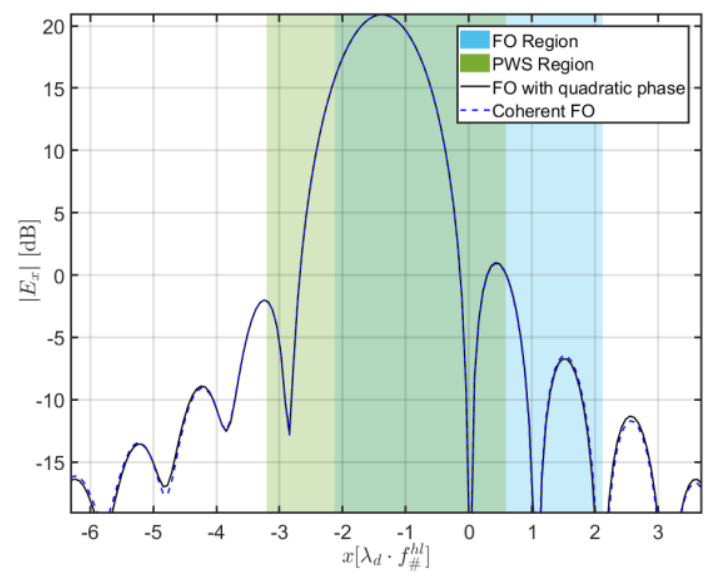

(a)

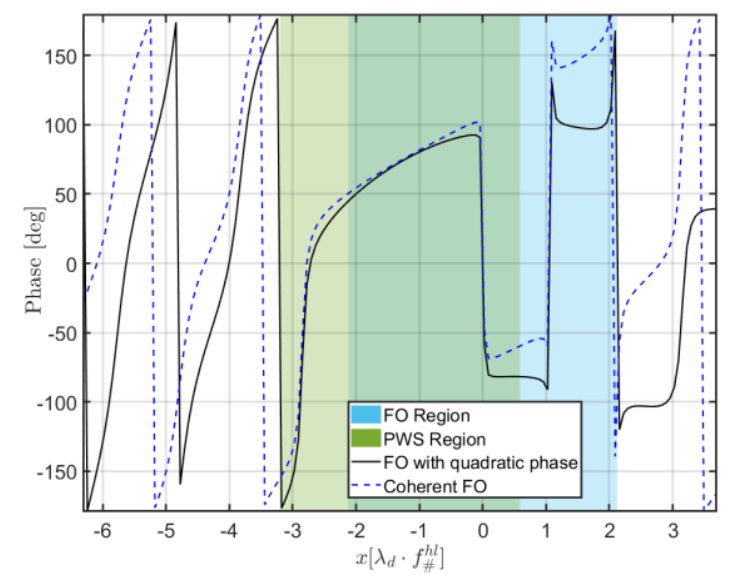

(b)

Figure 5.11: The $x$-component of the electric fields on the focal plane of a hemispherical silicon $\left(\varepsilon_{r}=11.9\right)$ lens with $D_{h l}=5 \lambda_{0}\left(f_{0}=300 \mathrm{GHz}\right), f_{\#}^{h l}=0.6, R_{s p h}=2.6 \lambda_{0}$, and $L=0.362 R_{s p h}$, illuminated by a unitary TM polarized plane wave with the skew angle of $\theta_{s}=15^{\circ}, \phi_{s}=0^{\circ}$. Here a quarter-wavelength matching layer at 300 $\mathrm{GHz}$ made of Parylene $\left(\varepsilon_{m}=2.62\right)$ is applied. The linearization point is chosen the same the flash point, i.e. $\vec{\rho}_{o} \approx$ $-1.3 \lambda_{d} f_{\#}^{h l} \hat{x}$. And the focal plane field calculated by using the coherent FO approach is compared with the one obtained using the GO-FO: (a) Amplitude. (b) Phase. One of the main planes $(y=0)$ is shown. Blue region is the FO applicability region and green region is the PWS applicability region. 


\section{- Hyperbolic lens}

A hyperbolic plastic $\left(\varepsilon_{r}=2\right)$ lens with $D_{h}=100 \lambda_{0}\left(f_{0}=300 \mathrm{GHz}\right)$ and $f_{\#}^{h}=1$ is introduced. The incident field is a unitary TM polarized plane wave with the skew angle of $\theta_{s}=3.2\left(\lambda_{d} / D_{h}\right)=1.3^{\circ}, \phi_{s}=0^{\circ}$, i.e. the flash point is $\vec{\rho}_{f p} \approx-3.2 \lambda_{0} f_{\#}^{h} \hat{x}$. The linearization point is chosen the as the flash point. Fig. 5.12 shows the $\mathrm{x}$-component of the electric fields on the focal plane of the hyperbolic lens. The focal plane field calculated by using the coherent FO is compared with the one obtained using the analytical FO. It can be seen that the coherent FO is validated inside the PWS applicability region.

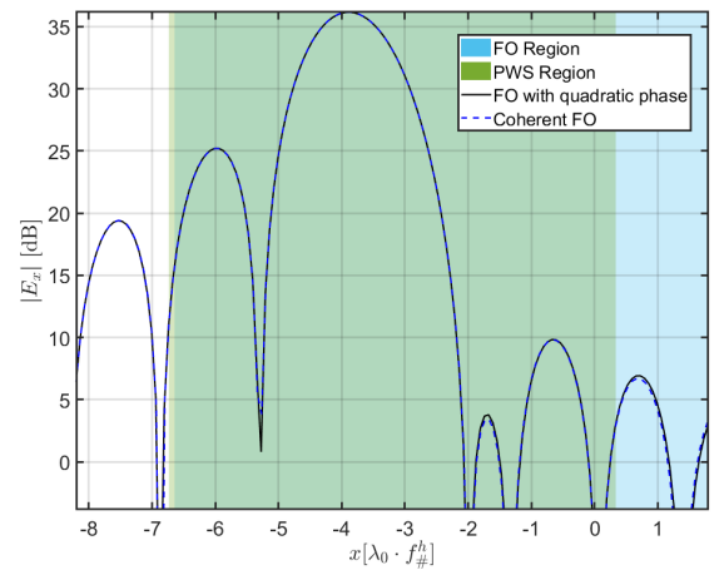

(a)

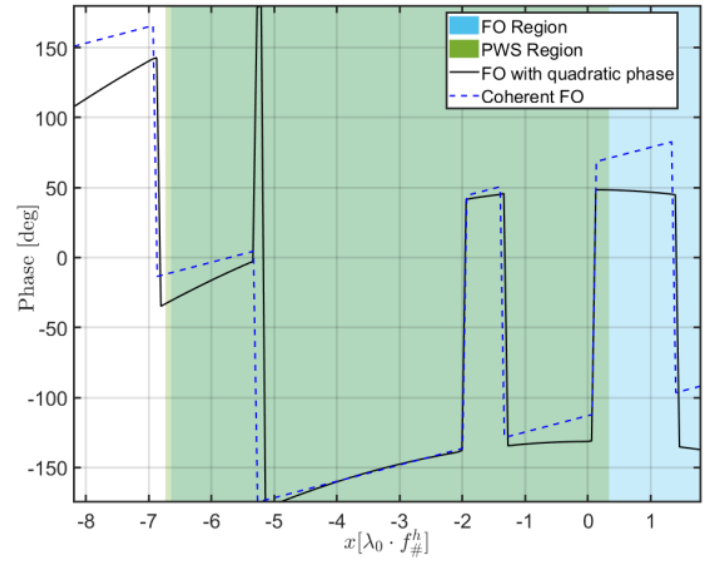

(b)

Figure 5.12: The $\mathrm{x}$-component of the electric fields on the focal plane of a hyperbolic plastic $\left(\varepsilon_{r}=2\right)$ lens with $D_{h}=100 \lambda_{0}\left(f_{0}=300 \mathrm{GHz}\right)$ and $f_{\#}^{h}=1$, illuminated by a unitary TM polarized plane wave with the skew angle of $\theta_{s}=3.2\left(\lambda_{d} / D_{h}\right)=1.3^{\circ}, \phi_{s}=0^{\circ}$. The linearization point is chosen the same the flash point, i.e. $\vec{\rho}_{o} \approx$ $-3.2 \lambda_{0} f_{\#}^{h} \hat{x}$. And the focal plane field calculated by using the coherent FO approach is compared with the one obtained using the analytical FO: (a) Amplitude. (b) Phase. One of the main planes $(y=0)$ is shown. Blue region is the FO applicability region and green region is the PWS applicability region. 
- Elliptical mirror

For an elliptical mirror, a source can be placed at either its upper focal plane or its lower focal plane. Here we consider a mirror with the diameter of $D_{m}=100 \lambda_{0}\left(f_{0}=300 \mathrm{GHz}\right)$, the semi-major axis of $a=80 \lambda_{0}$, and the focal distance of $c=20 \lambda_{0}$, i.e. the eccentricity is $e=$ 0.25 .

\section{Case 1: Source placed at the upper focal plane}

In this case, the f-number is calculated as $f_{\#}^{m}=0.95$. The incident field is generated by a unitary Huygens source placed at the upper focal plane, with the electric current oriented along $\hat{x}$ and a displacement in $\mathrm{x}$-direction, $x_{s}=2.2 \lambda_{0} f_{\#}^{m}$. This displacement corresponds to a flash point $\vec{\rho}_{f p} \approx-3.2 \lambda_{0} f_{\#}^{m} \hat{x}$ and the linearization point is the same as this flash point. Fig. 5.13 shows the $\mathrm{x}$-component of the electric fields on the focal plane of the mirror, evaluated by using the coherent FO and compared with the analytical FO. As it can be seen, the agreement is within the accepted error margin inside the PWS applicability region.

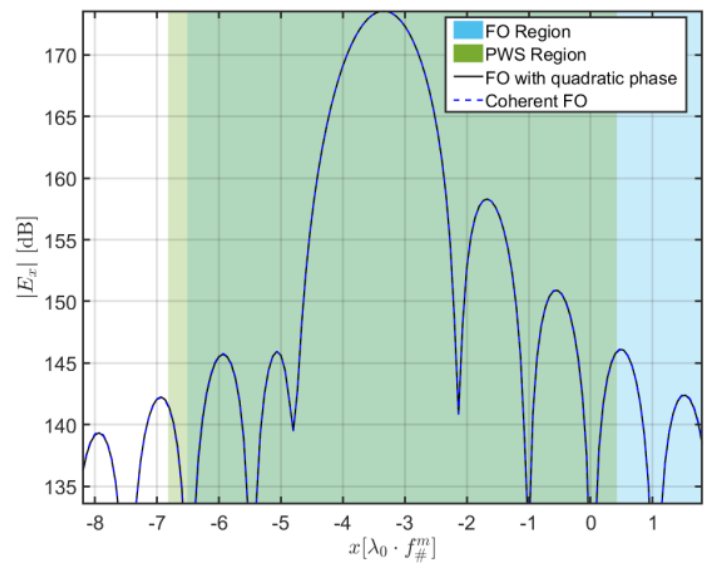

(a)

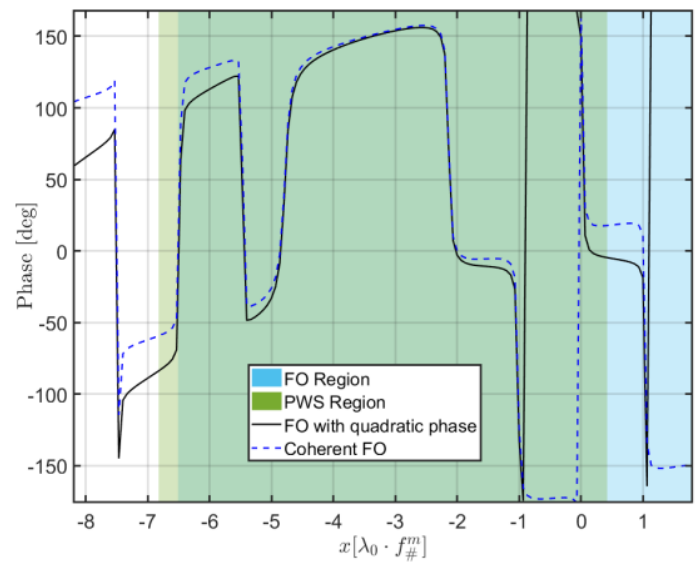

(b)

Figure 5.13: The $x$-component of the electric fields on the focal plane of an elliptical mirror with $D_{m}=$ $100 \lambda_{0}\left(f_{0}=300 \mathrm{GHz}\right), f_{\#}^{m}=0.95$ and $e=0.25$. The mirror is illuminated by a unitary Huygens source placed at the upper focal plane, with the electric current oriented along $\hat{x}$ and a displacement in $\mathrm{x}$-direction, $x_{s}=$ $2.2 \lambda_{0} f_{\#}^{m}$. The linearization point is chosen the same the flash point, i.e. $\vec{\rho}_{o} \approx-3.2 \lambda_{0} f_{\#}^{m} \hat{x}$. And the focal plane field calculated by using the coherent FO is compared with the one obtained using the analytical FO: (a) Amplitude. (b) Phase. One of the main planes $(y=0)$ is shown. Blue region is the FO applicability region and green region is the PWS applicability region. 


\section{Case 2: Source placed at the lower focal plane}

In this case, the f-number is calculated as $f_{\#}^{m}=0.6$. the Huygens source is placed at the lower focal plane, with the electric current oriented along $\hat{x}$ and a displacement in $\mathrm{x}$-direction, $x_{s}=4.7 \lambda_{0} f_{\#}^{m}$. This displacement corresponds to a flash point $\vec{\rho}_{f p} \approx-3.2 \lambda_{0} f_{\#}^{m} \hat{x}$ and the linearization point is the same as this flash point. Fig. 5.14 shows the $\mathrm{x}$-component of the electric fields on the focal plane of the mirror, evaluated by using the coherent FO and compared with the analytical FO. It can be seen that the coherent FO is in excellent agreement with the analytical FO inside the PWS applicability region.

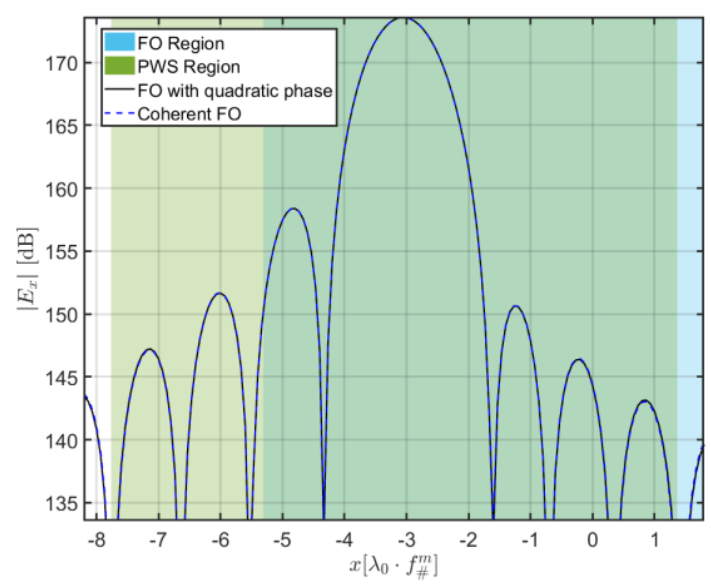

(a)

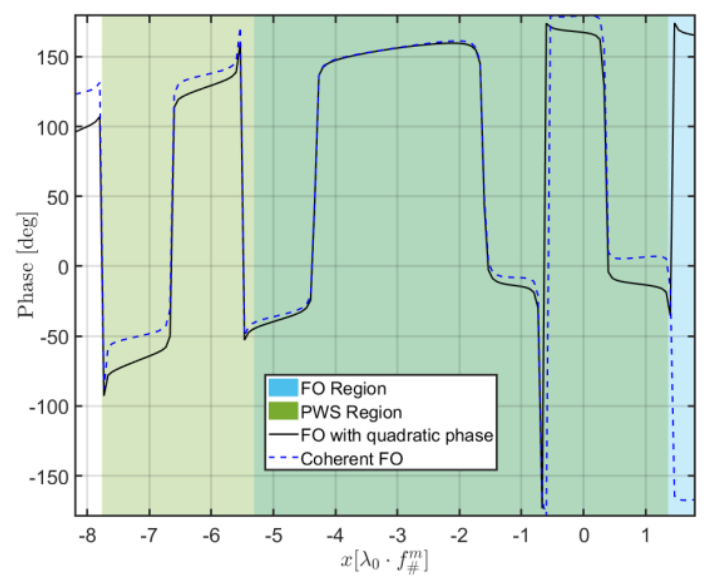

(b)

Figure 5.14: The $x$-component of the electric fields on the focal plane of an elliptical mirror with $D_{m}=$ $100 \lambda_{0}\left(f_{0}=300 \mathrm{GHz}\right), f_{\#}^{m}=0.6$ and $e=0.25$. The mirror is illuminated by a unitary Huygens source placed at the lower focal plane, with the electric current oriented along $\hat{x}$ and a displacement in $\mathrm{x}$-direction, $x_{s}=$ $4.7 \lambda_{0} f_{\#}^{m}$. The linearization point is chosen the same the flash point, i.e. $\vec{\rho}_{o} \approx-3.2 \lambda_{0} f_{\#}^{m} \hat{x}$. And the focal plane field calculated by using the coherent FO is compared with the one obtained using the analytical FO: (a) Amplitude. (b) Phase. One of the main planes $(y=0)$ is shown. Blue region is the FO applicability region and green region is the PWS applicability region. 


\section{Chapter 6: Antenna-coupled QO systems}

An important application of this thesis is to analyze and design antenna-coupled QO systems in reception. As will be shown in this chapter, for such a system, one can evaluate the power intercepted by the antenna and coupled to its load, by resorting to the Thevenin equivalent circuit [47] and antenna in reception formulism [17].

In this chapter, we analyze the performance of an antenna placed at the focal plane of a QO component illuminated by a generalized incident field. The antenna is assumed to be connected with a single-mode transmission line. Firstly, in section 6.1, we show the Thevenin equivalent circuit for the system in reception. We can evaluate the open-circuit voltage generator in this circuit by calculating a reaction integral; or under some approximations, by performing a field matching integral. Next in section 6.2, we explain explicitly how to calculate the power delivered to the antenna load. And then in section 6.3, we introduce parameters used to analyze the performance of an antenna in reception, including pattern, directivity, gain, and common efficiency terms. Finally, in section 6.4, the performance of antenna-coupled QO components is validated.

\subsection{Thevenin equivalent circuit and open-circuit voltage}

To analyze an antenna in reception, one needs to evaluate the power delivered to the load of the antenna. A good start point is the Thevenin equivalent circuit described in [47]. In this circuit, as will be discussed in subsection 6.1.1, an equivalent voltage generator is proposed for a generalized incidence. Once this voltage is known, one can estimate the power delivered to the load.

In order to calculate this voltage (subsection 6.1.2), a reaction integral between induction currents and fields in transmission is derived. Moreover, by introducing some approximations, one can express the voltage as a field matching between the incident GO field on a FO sphere and the field transmitted by the antenna. Based on this field matching integral, one can conclude that once these fields are conjugately matched, the power received by the antenna is maximized.

\subsubsection{Thevenin equivalent circuit}

We use the Thevenin equivalent circuit to estimate the power delivered to an antenna load, i.e. $P_{L}$. Fig. 6.1a shows the geometry of an antenna in reception. The antenna intercepts an incident 
field in a generic shape $\left(\vec{E}_{i}, \vec{H}_{i}\right)$ and delivers the incident power to its load through a singlemode transmission line. $\left(\vec{E}_{S}, \vec{H}_{S}\right)$ is the field scattered by the antenna and $\vec{E}_{o c}$ is the total field on the antenna gap. We select an arbitrary cross section $A A^{\prime}$ within the single-mode transmission line. If we separate the antenna and the load as two problems and use Schelkunoff's formulation of the equivalent theorem [48] at the cross section $A A^{\prime}$, after solving these two equivalent problems, we are able to derive the Thevenin equivalent circuit (Fig. 6.1b) for the reception scenario. Once we obtain the open-circuit voltage $V_{o c}$, we can evaluate the power dissipated by the load $Z_{L}$.

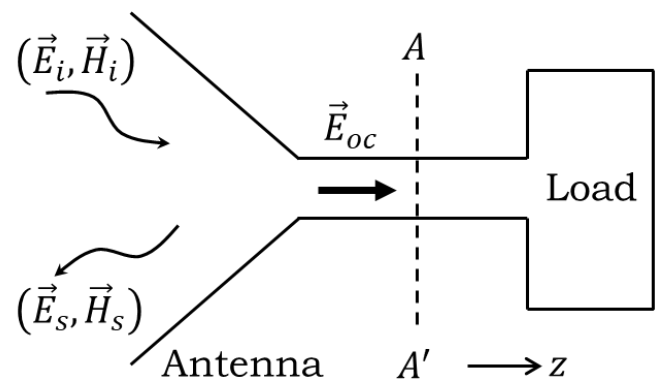

(a)

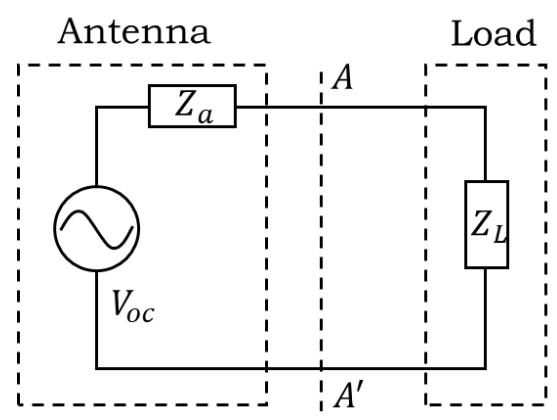

(b)

Figure 6.1: (a) An antenna intercepts an incident field and delivers its power to a load through a single-mode transmission line (b) The Thevenin equivalent circuit that models the incident field to the antenna as a voltage generator $V_{o c}$, the antenna as an impedance $Z_{a}$, and the load connected to the antenna as $Z_{l}$.

\subsubsection{Open-circuit voltage}

For a single-mode transmission line, the tangent components of electric and magnetic fields in the transmission line can be expressed as:

$$
\left\{\begin{array}{l}
\vec{E}_{0}=V_{0} \vec{e}_{0} \\
\vec{H}_{0}=I_{0} \vec{h}_{0}
\end{array}\right.
$$

where $\vec{e}_{0}$ and $\vec{h}_{0}$ are eigen vectors that are related to each other: $\vec{h}_{0}=\hat{n} \times \vec{e}_{0}, V_{0}$ and $I_{0}$ are modal amplitudes that can be obtained at a cross section $S_{C S}$ as:

$$
\left\{\begin{array}{c}
V_{0}=\iint_{S_{C S}} \vec{E}_{0} \cdot \vec{e}_{0} d S \\
I_{0}=\iint_{S_{C S}} \vec{H}_{0} \cdot \vec{h}_{0} d S
\end{array}\right.
$$

In the equivalent circuit, one can represent the open-circuit voltage at the cross section $S_{A A^{\prime}}$ as:

$$
V_{o c}=\iint_{S_{A A^{\prime}}} \vec{E}_{o c} \cdot \vec{e}_{0} d S
$$


Moreover, one can relate Eq. (6.3) to a transmitting antenna fed by an equivalent current $\vec{J}_{0}=$ $-\hat{z} \times \vec{H}_{0}$ at $A A^{\prime}$, by choosing an appropriate normal vector, $\hat{n}=-\hat{z}$, and multiplying $I_{0}$ at both sides of Eq. (6.3):

$$
V_{o c} I_{0}=\iint_{S_{A A^{\prime}}} \vec{E}_{O c} \cdot\left(I_{0} \vec{h}_{0} \times(-\hat{z})\right) d S=-\iint_{S_{A A^{\prime}}} \vec{E}_{o c} \cdot \vec{J}_{0} d S
$$

Therefore, Eq. (6.4) is now a reaction integral between two sources: the open-circuit voltage generator $V_{o c}$ representing the incoming field and the antenna equivalent current $\vec{J}_{0}$ in transmission. However, in this work, another form of Eq. (6.4) is desirable since $\vec{E}_{o c}$ and $\vec{J}_{0}$ are not straightforward quantities. Alternatively, we can represent Eq. (6.4) by another reaction integral, by resorting to the induction theorem [49] and the reciprocity theorem. To begin with, by using the induction theorem, one can assume $\vec{E}_{O c}$ is the field radiated by a set of currents $\vec{J}_{S i}$ and $\vec{M}_{S i}$ that are induced by the incident fields, $\left(\vec{E}_{i}, \vec{H}_{i}\right)$, on an arbitrary surface $S_{i}$. This surface is referred to as the induction surface that encircles the antenna and its surroundings. In the case of an antenna-coupled QO component, we can choose the induction surface as the FO sphere, as shown in Fig. 6.2. Accordingly, the incident fields are the GO fields described in chapter 4 , and the induction currents can be calculated as:

$$
\left\{\begin{array}{l}
\vec{J}_{G O}=\hat{n} \times \vec{H}_{G O} \\
\vec{M}_{G O}=\vec{E}_{G O} \times \hat{n}
\end{array}\right.
$$

where $\hat{n}=-\hat{r}^{\prime}$ is the normal vector of the FO sphere.

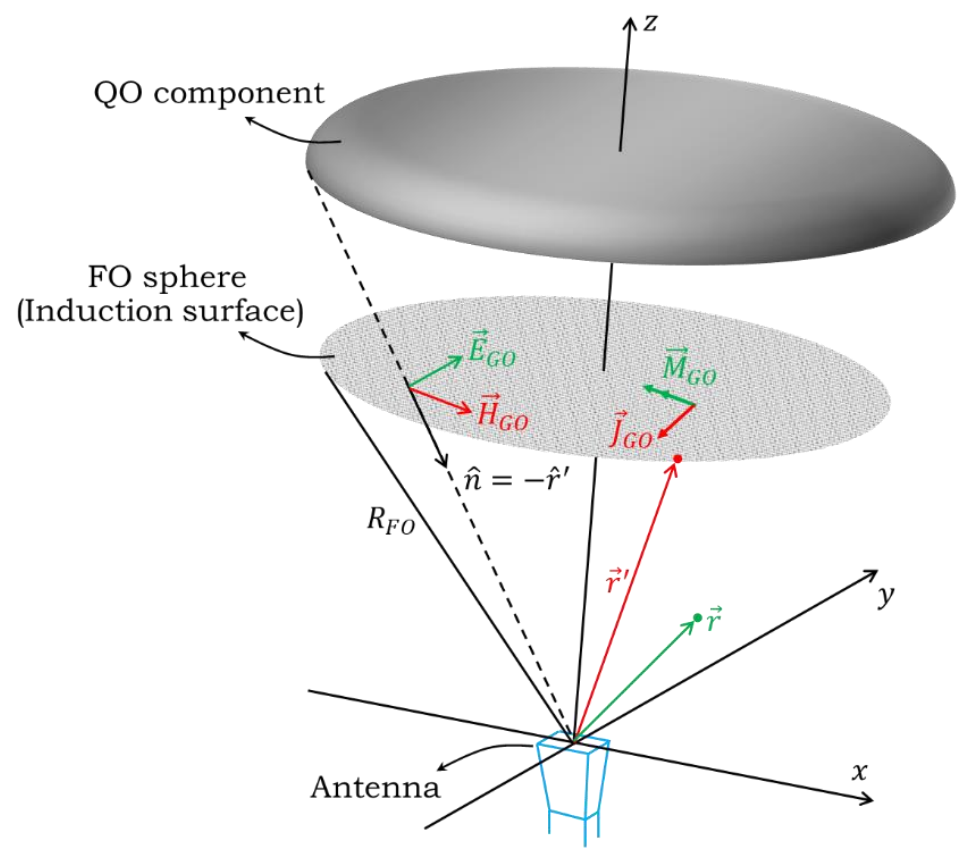

Figure 6.2: Induction surface is chosen the same as the equivalent FO sphere for an antenna-coupled QO component. 
Moreover, the open-circuit field, $\vec{E}_{o c}$, can be obtained by radiating the induction currents in the presence of the open circuited antenna and its surroundings:

$$
\vec{E}_{o c}(\vec{r})=\iint_{S_{F O}} \tilde{G}_{a}^{e j}\left(\vec{r}-\vec{r}^{\prime}\right) \vec{J}_{G O}\left(\vec{r}^{\prime}\right) d \vec{r}^{\prime}+\iint_{S_{F O}} \tilde{G}_{a}^{e m}\left(\vec{r}-\vec{r}^{\prime}\right) \vec{M}_{G O}\left(\vec{r}^{\prime}\right) d \vec{r}^{\prime}
$$

where $\tilde{G}_{a}^{e j}$ and $\tilde{G}_{a}^{e m}$ are the Green's functions in the presence of the open circuited antenna and its surroundings. One can also introduce the fields $\left(\vec{E}_{a}^{T x}, \vec{H}_{a}^{T x}\right)$ radiated by the current $\vec{J}_{0}$ in transmission and also in the presence of the antenna and its surroundings. Therefore, now we have two sets of sources that radiate with the same Green's functions: One is the induction currents that radiate $\vec{E}_{o c}$, the other is $\vec{J}_{0}$ that produces $\left(\vec{E}_{a}^{T x}, \vec{H}_{a}^{T x}\right)$. We can then apply the reciprocity theorem on Eq. (6.4), as depicted in Fig. 6.3, and calculate the reaction between the fields in transmission and the induction surface currents as follows:

$$
V_{o c}=\iint_{S_{F O}}\left[\vec{H}_{a}^{T x} \cdot \vec{M}_{G O}-\vec{E}_{a}^{T x} \cdot \vec{J}_{G O}\right] d S
$$

Here a unitary current amplitude in transmission is assumed, i.e. $I_{0}=1$.
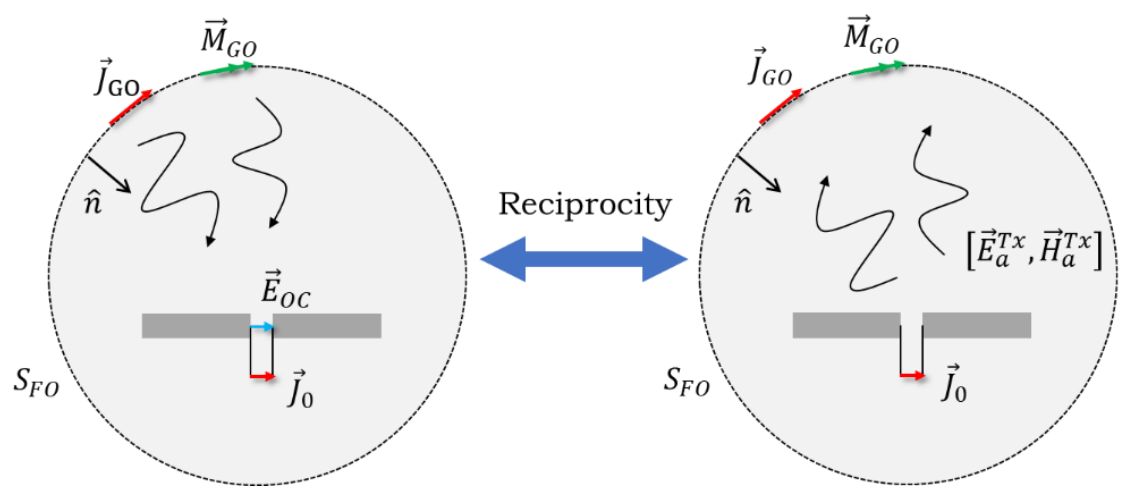

Figure 6.3: The reciprocity theorem between the induction surface currents $\left(\vec{J}_{G O}, \vec{M}_{G O}\right)$ and the equivalent current representing antenna in transmission $\vec{J}_{0}$.

Furthermore, if we assume the GO field behaves as a local plane wave and approximate the propagation unit vector, $\hat{s}_{G O}$, as $\hat{s}_{G O} \approx \hat{n}=-\hat{r}^{\prime}$, then we can relate the magnetic GO field to the electric GO field as:

$$
\vec{H}_{G O}\left(\vec{r}^{\prime}\right) \approx-\frac{1}{\zeta} \hat{r}^{\prime} \times \vec{E}_{G O}\left(\vec{r}^{\prime}\right)
$$

By substituting Eq. (6.8) in Eq. (6.7), and rewriting $d S$ as $R_{F O}^{2} \sin \theta d \theta d \phi$ :

$$
V_{o c}=\int_{0}^{2 \pi} \int_{0}^{\theta_{0}}\left[\frac{1}{\zeta} \vec{E}_{a}^{T x} \cdot \vec{E}_{G O}-\zeta \vec{H}_{a}^{T x} \cdot \vec{H}_{G O}\right] R_{F O}^{2} \sin \theta d \theta d \phi
$$


In most cases, the FO sphere is in the far-field region with respect to the antenna terminal; therefore, one can represent the fields transmitted by the antenna by its far fields, i.e. magnetic and electric fields are related to each other as:

$$
\vec{H}_{a}^{T x}\left(\vec{r}^{\prime}\right) \approx \frac{1}{\zeta} \hat{r}^{\prime} \times \vec{E}_{a}^{T x}\left(\vec{r}^{\prime}\right)
$$

By using Eq. (6.10), one can represent Eq. (6.9) as a field matching integral between the incident GO field and the far field of the antenna:

$$
V_{o c}=\frac{2}{\zeta} \int_{0}^{2 \pi} \int_{0}^{\theta_{0}} \vec{E}_{a}^{T x} \cdot \vec{E}_{G O} R_{F O}^{2} \sin \theta d \theta d \phi
$$

Eq. (6.11) indicates that when the antenna far field is conjugately matched with the incident GO field, i.e. $\vec{E}_{a}^{T x}=\left(\vec{E}_{G O}\right)^{*}$, the maximum open-circuit voltage can be achieved.

\subsection{Power delivered to an antenna load}

For an antenna-coupled QO system in reception, we have derived its Thevenin equivalent circuit and open-circuit voltage in section 6.1. Therefore, we are able to evaluate the power coupled to the load as:

$$
P_{L}=\frac{\left|V_{o c}\right|^{2}}{8 R_{a}} x_{m a t c h}
$$

where $R_{a}$ is the real part of the antenna impedance, $Z_{a}$, and $X_{\text {match }}=\frac{4 R_{L} R_{a}}{\left(R_{L}+R_{a}\right)^{2}+\left(X_{a}+X_{L}\right)^{2}}$ is the efficiency of impedance matching, where $Z_{L}=R_{L}+j X_{L}$ is the load impedance and $Z_{a}=$ $R_{a}+j X_{a}$ is the antenna impedance. Assuming an impedance matching condition for the load, i.e. $Z_{L}=Z_{a}^{*}$, Eq. (6.12) can be expressed as:

$$
P_{\text {load }}=\frac{\left|V_{o c}\right|^{2}}{8 R_{a}}
$$

Moreover, the antenna in transmission radiates the power, $P_{\text {rad }}=\frac{1}{2} R_{a}\left|I_{0}\right|^{2}$. When a unitary current is impressed, i.e. $I_{0}=1$, one can relate $R_{a}$ to $P_{\text {rad }}$ as:

$$
R_{a}=\frac{2 P_{\text {rad }}}{\left|I_{0}\right|^{2}}=2 P_{\text {rad }}
$$

By substituting Eq. (6.14) in Eq. (6.13), the power delivered to the load can be expressed as:

$$
P_{L}=\frac{\left|V_{o c}\right|^{2}}{16 P_{\text {rad }}}
$$


Moreover, $P_{\text {rad }}$ can be interpreted as the total power radiated by the antenna on the FO sphere:

$$
P_{\text {rad }}=\int_{0}^{2 \pi} \int_{0}^{\pi}\left|\vec{W}_{\text {rad }} \cdot \hat{r}^{\prime}\right| R_{F O}^{2} \sin \theta d \theta d \phi
$$

where $\vec{W}_{\text {rad }}$ is the time-average radiated power density:

$$
\vec{W}_{\text {rad }}=\frac{1}{2} \operatorname{Re}\left(\vec{E}_{a}^{T x} \times \vec{H}_{a}^{T x *}\right)
$$

By applying the expressions of $V_{o c}$ and $P_{r a d}$, one can express the coupled power for a generic case as follows:

$$
P_{L}=\frac{\left|\int_{0}^{2 \pi} \int_{0}^{\theta_{0}}\left[\vec{H}_{a}^{T x} \cdot \vec{M}_{G O}-\vec{E}_{a}^{T x} \cdot \vec{J}_{G O}\right] \sin \theta d \theta d \phi\right|^{2}}{16 \int_{0}^{2 \pi} \int_{0}^{\pi}\left|\vec{W}_{r a d} \cdot \hat{r}^{\prime}\right| \sin \theta d \theta d \phi}
$$

It is worth mentioning that this expression can be used also when the QO component is in the near field of the antenna and for very large skewed angles.

Furthermore, if one considers the approximations described in Eq. (6.8) and (6.10), Eq. (6.18) can be represented as the field matching between the filed in transmission and the GO field:

$$
P_{L}=\frac{\left|\int_{0}^{2 \pi} \int_{0}^{\theta_{0}} \vec{E}_{a}^{T x} \cdot \vec{E}_{G O} \sin \theta d \theta d \phi\right|^{2}}{2 \zeta \int_{0}^{2 \pi} \int_{0}^{\pi}\left|\vec{E}_{a}^{T x}\right|^{2} \sin \theta d \theta d \phi}
$$

For the case when the QO system is illuminated by an incident field from $\left(\theta_{S}, \phi_{S}\right)$ direction, the power delivered to the load of the antenna is represented by $P_{L}\left(\theta_{s}, \phi_{S}\right)$. Moreover, similar steps can be repeated for other incident skew angles. When the field in transmission, $\vec{E}_{a}^{T x}$, is significant only within the truncation angle $\theta_{0}$ and conjugately matched with the inward GO field, i.e. $\vec{E}_{a}^{T x}=\left(\vec{E}_{G O}\right)^{*}$, Eq. (6.19) can be simplified as follows:

$$
P_{L}=\frac{1}{2 \zeta} \int_{0}^{2 \pi} \int_{0}^{\theta_{0}}\left|\vec{E}_{a}^{T x}\right|^{2} \sin \theta d \theta d \phi=\frac{1}{2 \zeta} \int_{0}^{2 \pi} \int_{0}^{\theta_{0}}\left|\vec{E}_{G O}\right|^{2} \sin \theta d \theta d \phi=P_{\text {inw }}
$$

where $P_{i n w}$ is the inward incident power, i.e. the power of the GO fields crossing the FO sphere. As the result, a conclusion can be derived from Eq. (6.20) that once the field radiated by the antenna in transmission is conjugately matched with the GO field in reception, all inward incident power can be captured by the receiving antenna and delivered to the matched load; in other words, we are able to receive $100 \%$ of the inward incident power. When the fields are not fully matched, in order to evaluate $P_{L}$ efficiently, we consider two cases to calculate $\vec{E}_{G O}$ and $\vec{E}_{a}^{T x}$. 


\section{- Case 1: Slightly off-broadside incidence and slightly off-focus antenna}

In this case, we assume the skew angle is $\theta_{s} \leq 11^{\circ}$. Therefore, one can evaluate the GO fields analytically, as described in section 4.2.2:

$$
\vec{E}_{G O}\left(\theta_{S}, \phi_{S}\right) \simeq \vec{E}_{G O}\left(\theta_{s}=0\right) \mathrm{e}^{-j \vec{k}_{\rho} \cdot \vec{\rho}_{f p}} e^{-j \Phi_{c o m a}}\left(e^{-j \Phi_{c o m p}}\right)
$$

where $e^{-j \Phi_{\text {comp }}}$ is the compensation phase term for the case of elliptical mirrors. One can also displace an antenna within the focal plane of a QO component with a distance $\vec{d}_{a}$ from the center, as shown in Fig. 6.4. For small displacements, one can approximate the far field of the antenna as the one of a central feed multiplied by a progressive phase term:

$$
\vec{E}_{a}^{T x}\left(\vec{d}_{a}\right) \simeq \vec{E}_{a}^{T x}\left(\vec{d}_{a}=0\right) e^{j \vec{k}_{\rho} \cdot \vec{d}_{a}}
$$

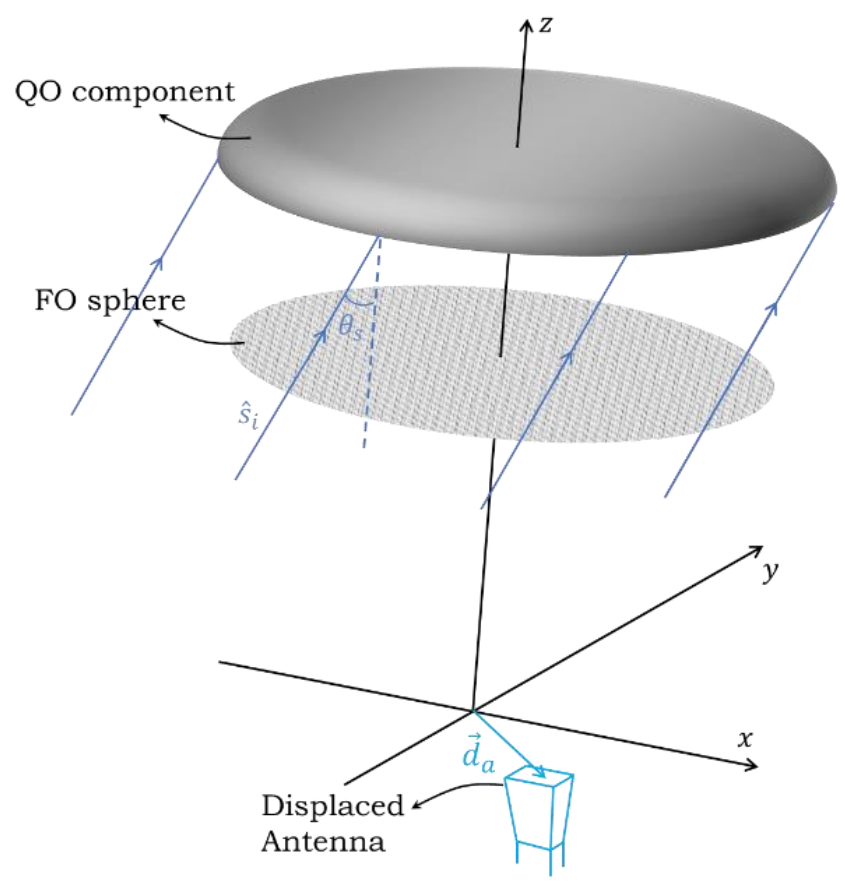

Figure 6.4: A QO component illuminated by incident fields with the skew angle of $\left(\theta_{s}, \phi_{s}\right)$. A receiving antenna is placed on the focal plane of the QO component with a displacement $\vec{d}_{a}$ from the center.

By substituting Eq. (6.21) and (6.22) in the field matching integral, Eq. (6.11), one can represent $V_{o c}$ as a function of the skew angle $\left(\theta_{s}, \phi_{s}\right)$ and the displacement of the antenna $\vec{d}_{a}$ :

$$
\begin{aligned}
V_{o c}\left(\theta_{s}, \phi_{s}, \vec{d}_{a}\right)=\frac{2}{\zeta} & \int_{0}^{2 \pi} \int_{0}^{\theta_{0}} \vec{E}_{a}^{T x}\left(\vec{d}_{a}=0\right) \cdot \vec{E}_{G O}\left(\theta_{s}=0\right) \cdot \\
& e^{j \vec{k}_{\rho} \cdot \vec{d}_{\mathrm{a}}} \mathrm{e}^{-j \vec{k}_{\rho} \cdot \vec{\rho}_{f p}} e^{-j \Phi_{c o m a}}\left(e^{-j \Phi_{c o m p}}\right) R_{F O}^{2} \sin \theta d \theta d \phi
\end{aligned}
$$

In Eq. (6.23), if the skew angle is smaller than the limit of the coma phase, i.e. $e^{-j \Phi_{\text {coma }} \approx} \approx$ 1 , and the compensation phase term can be neglected for the case of elliptical mirrors, i.e. 
$e^{-j \Phi_{\text {comp }}} \approx 1$, then $V_{o c}\left(\theta_{s}, \phi_{s}, \vec{d}_{a}\right)$ is maximum when $\vec{d}_{\mathrm{a}}=\vec{\rho}_{f p}$. In other words, the coupled power $P_{L}$ can be maximized from a certain skew incidence if one places the antenna feed at the corresponding flash point position.

\section{- Case 2: Large-angle incidence and large displacement of an antenna}

In cases when the skew angle is $\theta_{s} \geq 11^{\circ}$, one needs to calculate the GO ray field described in section 4.1. Moreover, when the displacement of the antenna, $\vec{d}_{a}$, is large so that one cannot use the approximation in Eq. (6.22), the field radiated by the antenna, $\vec{E}_{a}^{T x}\left(\vec{d}_{a}\right)$, should be evaluated numerically, which will be explicitly discussed in chapter 7, section 7.5.2.

\subsection{Performance of antenna-coupled QO systems in reception}

One can evaluate the performance of an antenna-coupled QO system in reception by analyzing its pattern, directivity, gain, and common efficiency terms.

\section{- Power pattern in reception}

One can define the power pattern in reception for an antenna-coupled QO system as:

$$
F_{R x}\left(\theta_{s}, \phi_{s}\right)=\frac{P_{L}\left(\theta_{s}, \phi_{s}\right)}{\max \left(P_{L}\left(\theta_{s}, \phi_{s}\right)\right)}
$$

where $F_{R x}\left(\theta_{s}, \phi_{s}\right)$ is the pattern for one skew angle $\left(\theta_{s}, \phi_{s}\right)$. If one wants to obtain the complete pattern in reception, one needs to illuminate the QO component by incident fields with different skew angles: $\theta_{s} \in\left[0, \theta_{R x}\right], \phi_{s} \in[0,2 \pi]$. This pattern indicates how much power is coupled to a load as a function of the incident skew angle, and due to the reciprocity, it should be the same as the far-field pattern of the same antenna in transmission. Therefore, one can compare the pattern in transmission with the one in reception to validate the procedure. And examples of validation will be discussed in section 6.4 for each QO component. Moreover, one can evaluate the directivity using the pattern in reception, $F_{R x}\left(\theta_{S}, \phi_{S}\right)$, as:

$$
D_{i r}^{R x}=\max \left(4 \pi \frac{F_{R x}\left(\theta_{s}, \phi_{s}\right)}{P_{R x}\left(\theta_{s}, \phi_{s}\right)}\right)
$$

where $P_{R x}$ is calculated by integrating the pattern in reception:

$$
P_{R x}=\int_{0}^{2 \pi} \int_{0}^{\theta_{R x}} F_{R x}\left(\theta_{s}, \phi_{s}\right) \sin \theta_{s} d \theta_{s} d \phi_{s}
$$


- Common efficiency terms

Besides the pattern, efficiency terms are also important parameters when analyzing the performance. Common efficiency terms are aperture efficiency, taper efficiency and spillover efficiency. The aperture efficiency of an antenna in reception can be expressed as a ratio between the coupled power to the load and the incident power:

$$
\eta_{a p}^{R x}=\frac{P_{L}}{P_{\text {inc }}}
$$

The incident power in Eq. (6.27), i.e. $P_{\text {inc }}$, for a plane wave incidence, can be expressed as:

$$
P_{i n c}=\frac{\left|E_{0}\right|^{2}}{2 \zeta} A_{Q O}
$$

where $E_{0}$ is the amplitude of the plane wave, $\zeta$ is the impedance of the medium, and $A_{Q O}$ is the area of the QO component. For a point-source incidence, $P_{i n c}$ is calculated as follows:

$$
P_{i n c}=\int_{0}^{2 \pi} \int_{0}^{\theta_{0}}\left|\vec{W}_{i n c} \cdot \vec{n}\right| d \theta d \phi
$$

where $\vec{W}_{i n c}=\frac{1}{2} \operatorname{Re}\left(\vec{E}_{i} \times \vec{H}_{i}\right)$ is the time-average radiated power density and $\vec{n}$ is the normal vector of the QO component. By using the aperture efficiency, one can also obtain the gain in reception:

$$
G_{a i n}^{R x}=D_{i r}^{\max } \eta_{a p}^{R x}
$$

where $D_{i r}^{\max }=\frac{4 \pi}{\lambda^{2}} A_{Q O}$ is the maximum theoretical directivity, which is achieved by a constant illumination of the QO surface in transmission. The taper efficiency in reception can be calculated as the ratio between the achieved directivity and the maximum theoretical directivity:

$$
\eta_{t}^{R x}=\frac{D_{i r}^{R x}}{D_{i r}^{\max }}
$$

Finally, the spillover efficiency in reception is the ratio between the aperture efficiency and the taper efficiency:

$$
\eta_{s o}^{R x}=\eta_{a p}^{R x} / \eta_{t}^{R x}
$$




\subsection{Validation of the performance in reception}

In this section, we validate the performance of antenna-coupled QO components in reception, including pattern, aperture efficiency, directivity, and gain. Due to the reciprocity, one can use the performance in transmission as the reference to validate the performance in reception. Here the power delivered to an antenna load is evaluated by using Eq. (6.18).

First in subsection 6.4.1, we introduce an approach to display pattern in reception. And then in subsection 6.4.2, examples of validation are shown for all QO components.

\subsubsection{Display a pattern in reception}

The power pattern in reception is a function of skew angles, i.e. $F_{R x}\left(\theta_{s}, \phi_{s}\right)$, as described in Eq. (6.24). To describe the pattern, one can select three $2 \mathrm{D}$ cuts: $\phi_{s}=0^{\circ} / 45^{\circ} / 90^{\circ}$. For each $\phi_{s}$ cut, the pattern is a function of $\theta_{s}$, as depicted in Fig. 6.5a. However, displaying a pattern for a scanning scenario is not convenient in such a matter. Since the center of the coordinate system is located at $\left(\theta_{s}=0^{\circ}, \phi_{s}=0^{\circ}\right), \phi_{s}=0^{\circ} / 45^{\circ} / 90^{\circ}$ cuts may not cross the main lobe of the pattern, as depicted in Fig. 6.5b with the dashed lines. In such cases, we introduce a local system. As it can be seen in Fig. 6.5b, we move the center of the coordinate system to the main lobe position, i.e. $\left(u_{c}, v_{c}\right)$, and display the pattern by using $u_{s} / v_{s} / \rho_{s}$ cuts, where $u_{s}=$ $\sin \theta_{s} \cos \phi_{s}, v_{s}=\sin \theta_{s} \sin \phi_{s}$, and $\rho_{s}=\sqrt{2} u_{s}$. By introducing this local system, one is able to display the pattern within a rectangular region that encloses the area around the main lobe. Moreover, in most practical cases with semi-symmetric patterns, $u_{s} / v_{s} / \rho_{s}$ cuts are sufficient to describe the whole pattern.

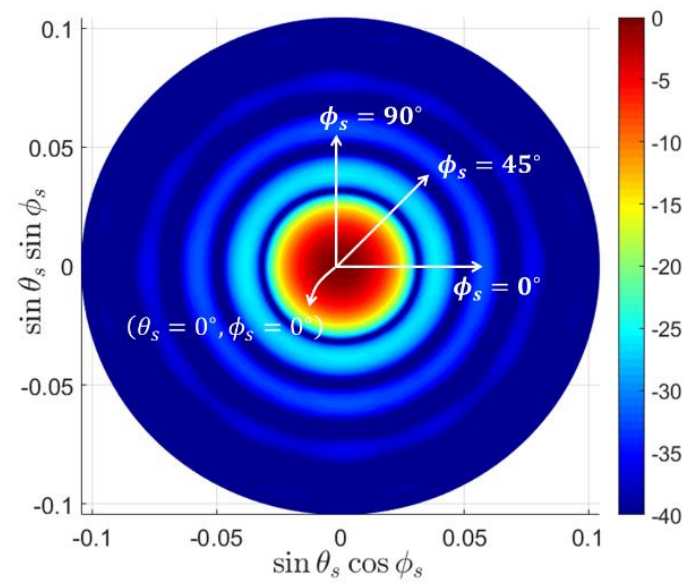

(a)

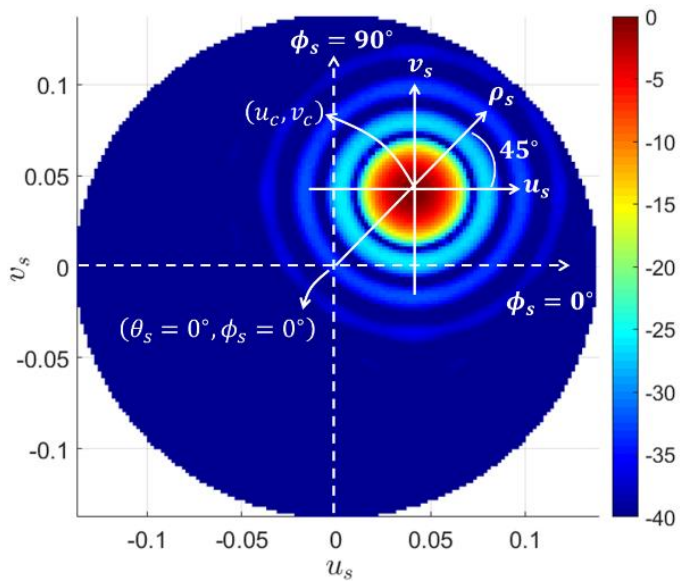

(b)

Figure 6.5: Pattern in reception: (a) Pattern centered at $\left(\theta_{s}=0^{\circ}, \phi_{s}=0^{\circ}\right)$, with $\phi_{s}=0^{\circ} / 45^{\circ} / 90^{\circ}$ cuts. (b) Pattern shifted to $\left(u_{c}, v_{c}\right)$, with $u_{s} / v_{s} / \rho_{s}$ cuts. 


\subsubsection{Examples of validation for all QO components}

In this subsection, all antenna-coupled QO components in reception scenarios are analyzed and validated. A leaky-wave slot is studied here as the receiving antenna. When it is integrated with a dielectric lens, as depicted in Fig. 6.6, the structure is referred to as the leaky lens antenna [50]. The fields radiated by the leaky-wave slot are exported from CST. While the fields transmitted out of the lens are calculated by using a developed PO code in transmission.

Here we use Eq. (6.18) to evaluate the power received by an antenna. In Eq. (6.18), the induction currents on a FO sphere can be calculated by using the GO fields discussed in chapter 4. The fields radiated by the antenna on the FO sphere are evaluated by using the antenna propagation approach, which will be discussed in the following chapter, section 7.5.2. The power pattern, the aperture efficiency, and the gain are described in Eq. (6.24), (6.27), and (6.30), respectively. For the directivity, we use an efficient way to calculate it, which will be discussed in section 7.5.3, Eq. (7.30).

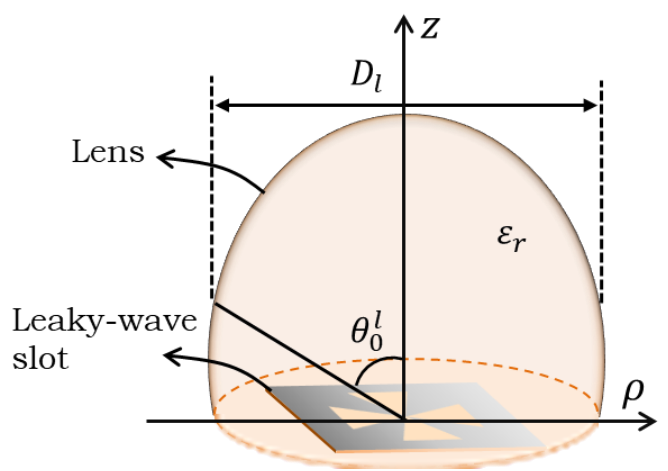

Figure 6.6: A schematic representation of a leaky lens antenna.

\subsubsection{Parabolic reflector}

In the case of a parabolic reflector, we place a leaky lens antenna at its focal plane as the receiving antenna that intercepts the fields focalized by it. Here we introduce a reflector with the diameter of $D_{r}=0.125 \mathrm{~m}$ and the f-number of $f_{\#}^{r}=1.5\left(\theta_{0}^{r}=19^{\circ}\right)$, illuminated by unitary Co-Pol. and Cx-Pol. plane waves; and a leaky lens antenna that consists of an elliptical silicon $\left(\varepsilon_{r}=11.9\right)$ lens with $D_{l}=1.3 \mathrm{~mm}$ and $f_{\#}^{l}=0.526\left(\theta_{0}^{l}=72^{\circ}\right)$ and a y-polarized leaky-wave slot operated at $720 \mathrm{GHz}$ [51]. The leaky lens antenna is shifted from the center of the focal plane with the distance $\vec{d}_{a}=17.5 \mathrm{~mm}$, as depicted in Fig. 6.7. 


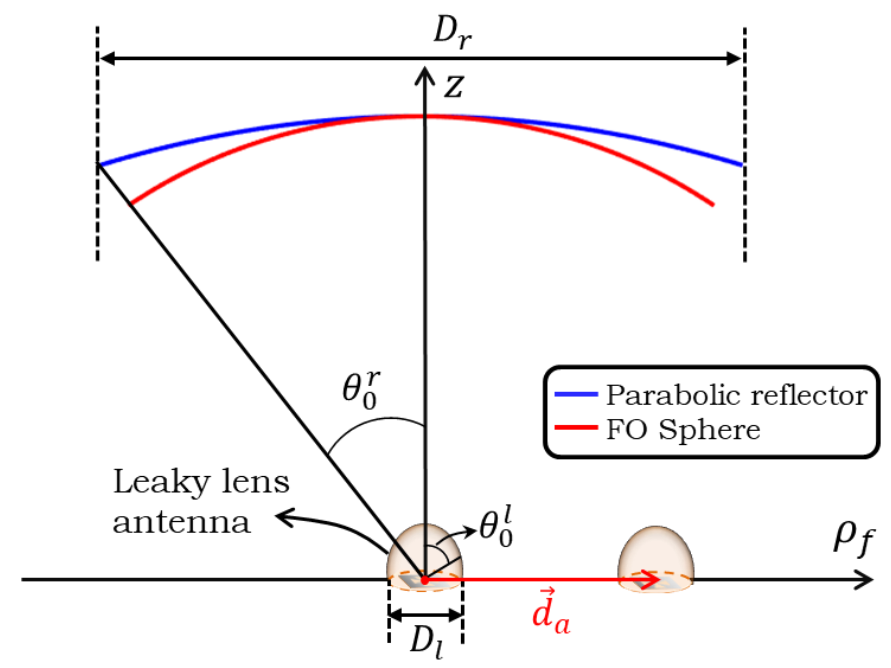

Figure 6.7: Geometry of an antenna-coupled (leaky lens antenna) parabolic reflector. The reflector has the diameter of $D_{r}=0.125 \mathrm{~m}$ and the f-number of $f_{\#}^{r}=1.5\left(\theta_{0}^{r}=19^{\circ}\right)$, illuminated by unitary Co-Pol. and Cx-Pol. plane waves. At its focal plane, the leaky lens antenna is shifted $\vec{d}_{a}=17.5 \mathrm{~mm}$ from the center. The antenna consists of an elliptical silicon $\left(\varepsilon_{r}=11.9\right)$ lens with $D_{l}=1.3 \mathrm{~mm}$ and $f_{\#}^{l}=0.526\left(\theta_{0}^{l}=72^{\circ}\right)$, and a y-polarized leaky-wave slot operated at $720 \mathrm{GHz}$.

Fig. 6.8 shows the resulting pattern in reception $(\mathrm{Rx})$, compared with the pattern in transmission (Tx). In this case, when calculating the pattern in $\mathrm{Rx}$, the far fields radiated by the leaky lens antenna are evaluated by using the PO in transmission code. The pattern in Tx is obtained by importing the far-field pattern of the same leaky lens antenna into GRASP. Fig. 6.8a to Fig. 6.8c show $u_{s}, \rho_{s}$, and $v_{s}$ planes, respectively; and Fig. $6.8 \mathrm{~d}$ is the 3D Co-Pol. pattern in Rx. As it can be seen, the pattern in Rx is in excellent agreement with the pattern in Tx, for both the Co-pol. and the Cx-pol. components. Moreover, the aperture efficiency, the directivity and the gain in Rx are well validated, as shown in Table. 6.1.

\begin{tabular}{|c|c|c|c|}
\hline Frequency: $\mathbf{7 2 0} \mathbf{~ G H z}$ & Aperture Efficiency & Directivity [dB] & Gain [dB] \\
\hline $\mathrm{Tx}$ & $29.64 \%$ & 58.51 & 54.20 \\
\hline $\mathrm{Rx}$ & $29.40 \%$ & 58.47 & 54.17 \\
\hline
\end{tabular}

Table 6.1: Performance of the antenna-coupled (leaky lens antenna) parabolic reflector, evaluated in Rx and compared with the one in Tx. 


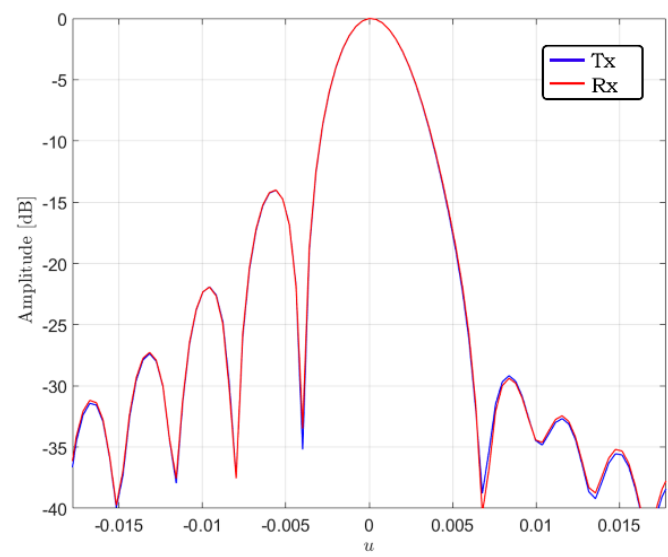

(a)

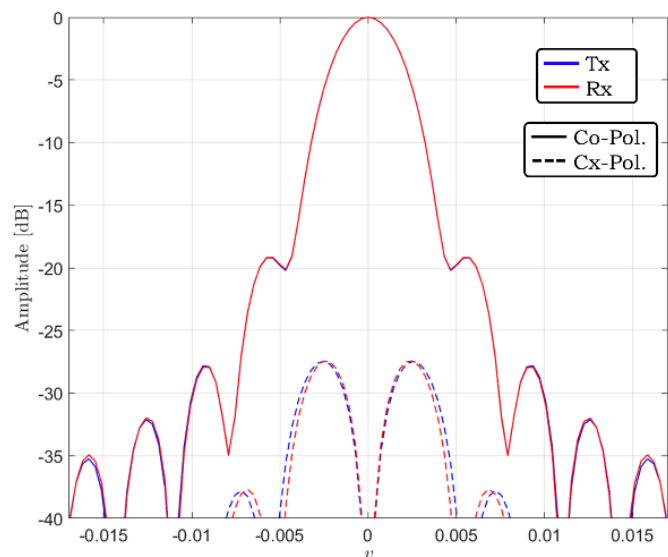

(c)

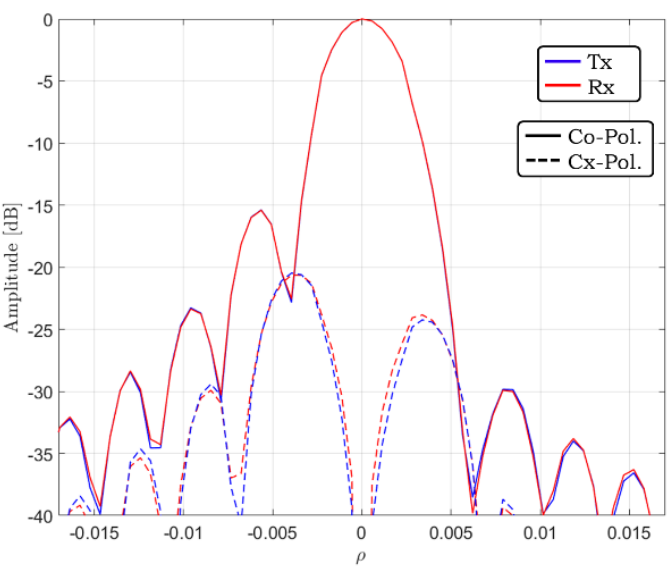

(b)

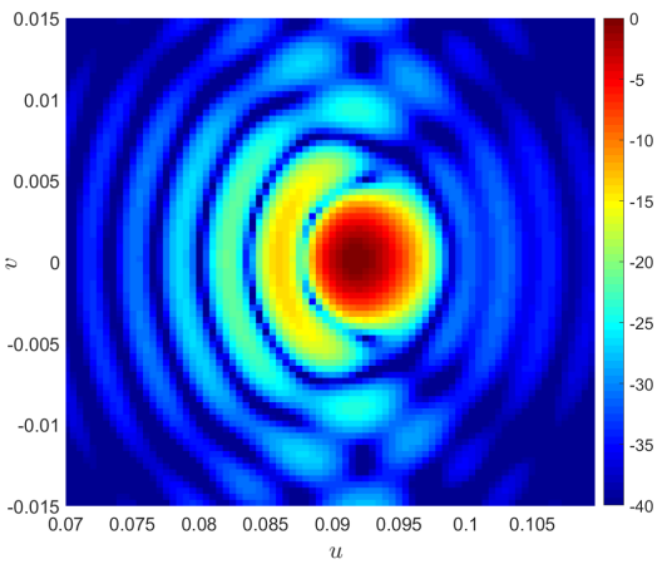

(d)

Figure 6.8: The pattern obtained in Rx of the antenna-coupled (leaky lens antenna) parabolic reflector, compared with the pattern evaluated in Tx: (a) $u_{s}$ plane. (b) $\rho_{s}$ plane. (c) $v_{s}$ plane. (d) 3D Co-Pol. pattern in Rx.

\subsubsection{Elliptical lens}

In the case of an elliptical lens, we analyze two types of leaky lens antennas. In the first case, a feed is placed close to the lens base, i.e. with an electrically small air gap $h$ (Fig. 6.9a). In the second case, a feed is placed $\lambda / 2$ away from the lens base (Fig. 6.9b), which is referred to as the resonant leaky lens antenna [52]. The difference between these two lenses is that the first lens is in the far-field region of the feed while the second lens is in the near-field region. The feed can be shifted with a distance $\vec{d}_{a}$ from the broadside position, as shown in Fig. 6.9. 


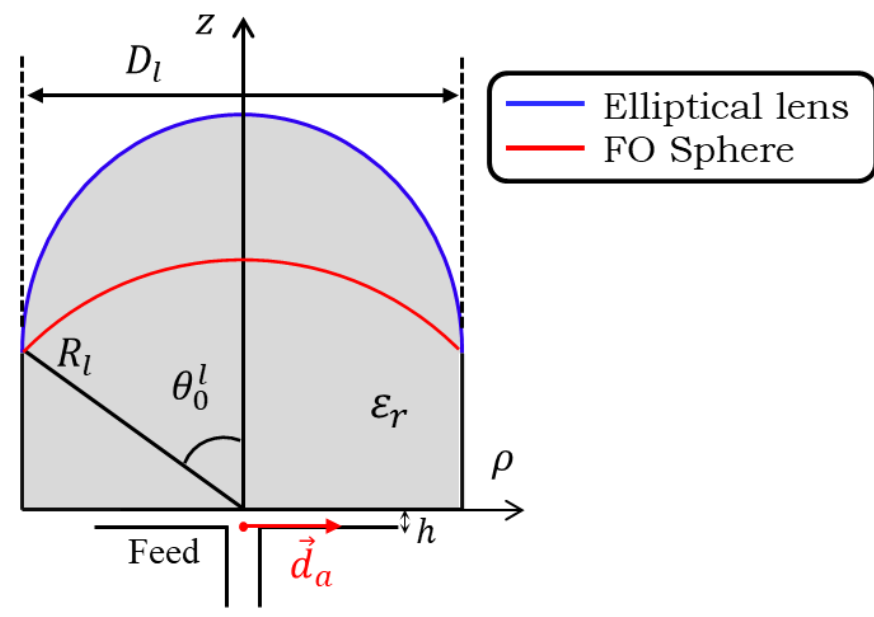

(a)

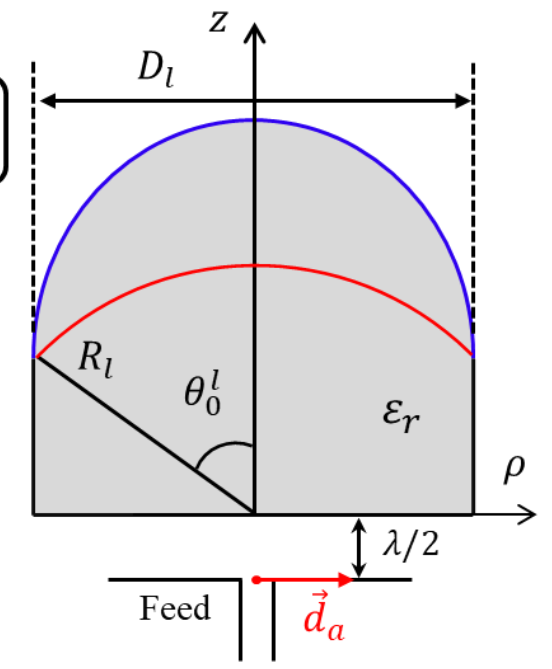

(b)

Figure 6.9: Antenna-coupled elliptical lenses: (a) leaky lens antenna. (b) Resonant leaky lens antenna.

\section{- Case 1: Leaky lens antenna}

In this case, we place a leaky-wave slot below an elliptical lens as the receiving antenna that intercepts the fields focalized by the lens. The lens is made of silicon $\left(\varepsilon_{r}=11.9\right)$, with $D_{l}=$ $5 \mathrm{~mm}$ and $f_{\#}^{l}=0.526\left(\theta_{0}^{l}=72^{\circ}\right)$, illuminated by unitary Co-Pol. and Cx-Pol. plane waves. Here a quarter-wavelength matching layer at $480 \mathrm{GHz}$ made of Parylene $\left(\varepsilon_{m}=2.62\right)$ is applied. The feed antenna is y-polarized and operated at $720 \mathrm{GHz}$, shifted from the broadside position with the distance $\vec{d}_{a}=0.11 \mathrm{~mm}$. Fig. 6.10 shows the pattern obtained in $\mathrm{Rx}$, compared with the pattern in Tx. In this case, the FO sphere is in the far-field region of the antenna. When calculating the pattern in Rx, the antenna far fields are exported from CST and are calculated on the FO sphere by using the antenna propagation approach. The pattern in $\mathrm{Tx}$ is the far field transmitted out of the lens, which is evaluated by using the PO in transmission code. Fig. 6.10a to Fig. 6.10c show $u_{s}, \rho_{s}$, and $v_{s}$ planes, respectively; and Fig. 6.10d is the 3D Co-Pol. pattern in Rx. As it can be seen, the pattern in Rx is in good agreement with the pattern in Tx, for both the Co-pol. and the Cx-pol. components. Moreover, the aperture efficiency, the directivity and the gain in Rx are validated, as shown in Table. 6.2.

\begin{tabular}{|c|c|c|c|}
\hline Frequency: 720 GHz & Aperture Efficiency & Directivity [dB] & Gain [dB] \\
\hline $\mathrm{Tx}$ & $33.55 \%$ & 27.56 & 26.78 \\
\hline $\mathrm{Rx}$ & $34.56 \%$ & 27.69 & 26.91 \\
\hline
\end{tabular}

Table 6.2: Performance of the leaky lens antenna evaluated in Rx and compared with the one in Tx. 


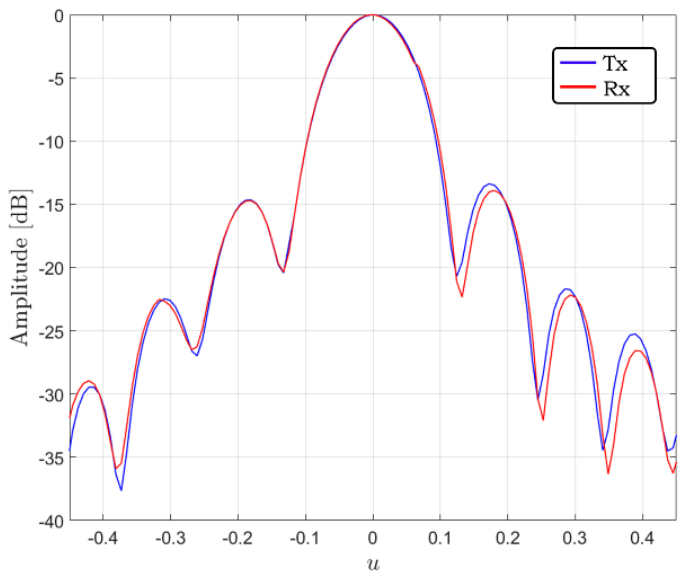

(a)

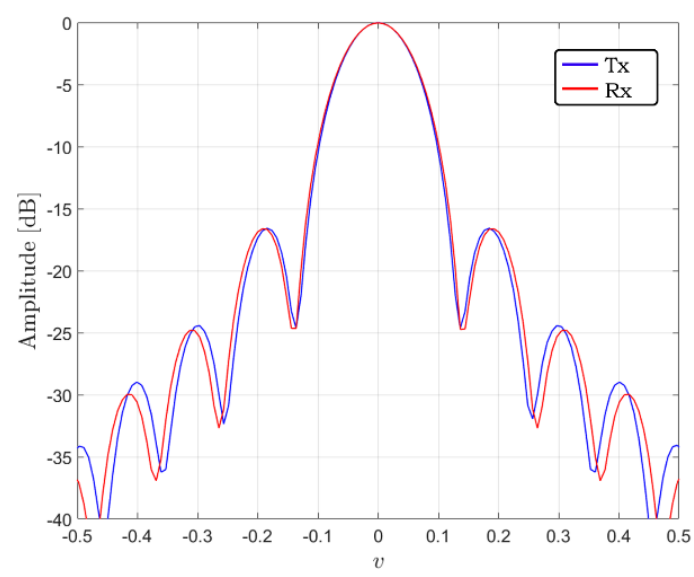

(c)

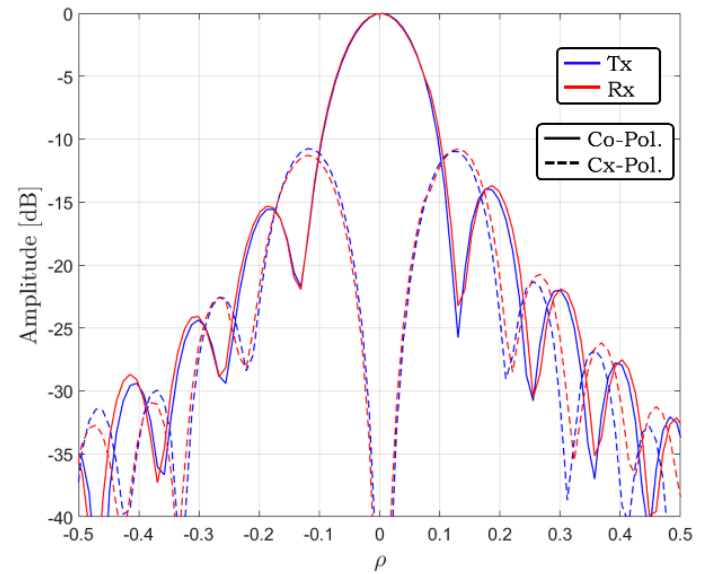

(b)

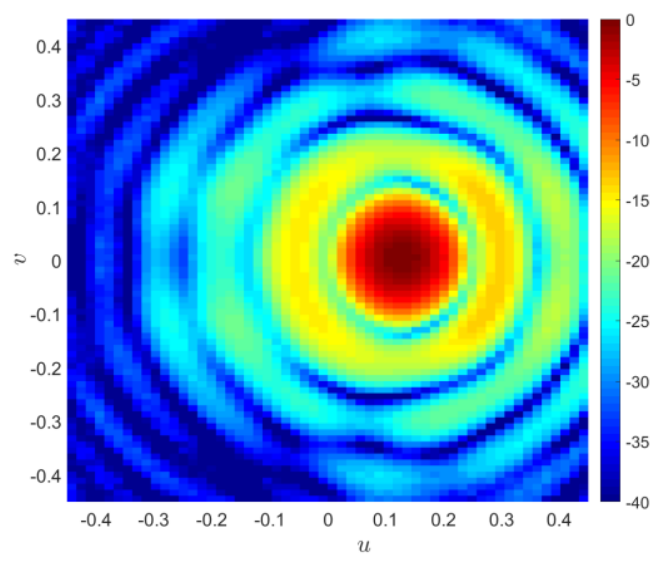

(d)

Figure 6.10: The pattern obtained in Rx of a leaky lens antenna, compared with the pattern evaluated in Tx: (a) $u_{s}$ plane. (b) $\rho_{s}$ plane. (c) $v_{s}$ plane. (d) 3D Co-Pol. pattern in Rx. The elliptical lens is made of silicon $\left(\varepsilon_{r}=11.9\right)$, with $D_{l}=5 \mathrm{~mm}$ and $f_{\#}^{l}=0.526\left(\theta_{0}^{l}=72^{\circ}\right)$, illuminated by unitary Co-Pol. and Cx-Pol. plane waves. Here a quarter-wavelength matching layer at $480 \mathrm{GHz}$ made of Parylene $\left(\varepsilon_{m}=2.62\right)$ is applied. The feed antenna is $\mathrm{y}-$ polarized and operated at $720 \mathrm{GHz}$, shifted from the broadside position with the distance $\vec{d}_{a}=0.11 \mathrm{~mm}$.

\section{- Case 2: Resonant leaky lens antenna}

In this case, an elliptical silicon $\left(\varepsilon_{r}=11.9\right)$ lens has the diameter of $D_{l}=2.78 \mathrm{~mm}$ and the $\mathrm{f}$ number of $f_{\#}^{l}=1.54\left(\theta_{0}^{l}=19^{\circ}\right)$, illuminated by unitary Co-Pol. and Cx-Pol. plane waves. The feed antenna is x-polarized and operated at $540 \mathrm{GHz}$, shifted from the broadside position with the distance $\vec{d}_{a}=0.375 \mathrm{~mm}$. Fig. 6.11 shows the pattern obtained in Rx, compared with the pattern in Tx. In this case, the FO sphere is in the near-field region of the antenna. When calculating the pattern in Rx, the antenna near fields are exported from CST and are calculated on the FO sphere by using the antenna propagation approach. The pattern in Tx is the far field transmitted out of the lens, which is evaluated by CST simulation. Fig. 6.11a to Fig. 6.11c show 
$u_{s}, \rho_{s}$, and $v_{s}$ planes, respectively; and Fig. $6.11 \mathrm{~d}$ is the 3D Co-pol. pattern in Rx. It can be seen that the pattern in Rx is in fair agreement with the pattern in Tx, for both the Co-pol. and the Cx-pol. components. Moreover, the aperture efficiency, the directivity and the gain in $\mathrm{Rx}$ are validated, as shown in Table. 6.3.

\begin{tabular}{|c|c|c|c|}
\hline Frequency: 540 GHz & Aperture Efficiency & Directivity [dB] & Gain [dB] \\
\hline $\mathrm{Tx}$ & $41.00 \%$ & 22.70 & 20.05 \\
\hline $\mathrm{Rx}$ & $45.25 \%$ & 22.86 & 20.48 \\
\hline
\end{tabular}

Table 6.3: Performance of the resonant leaky lens antenna, evaluated in Rx and compared with the one in Tx.

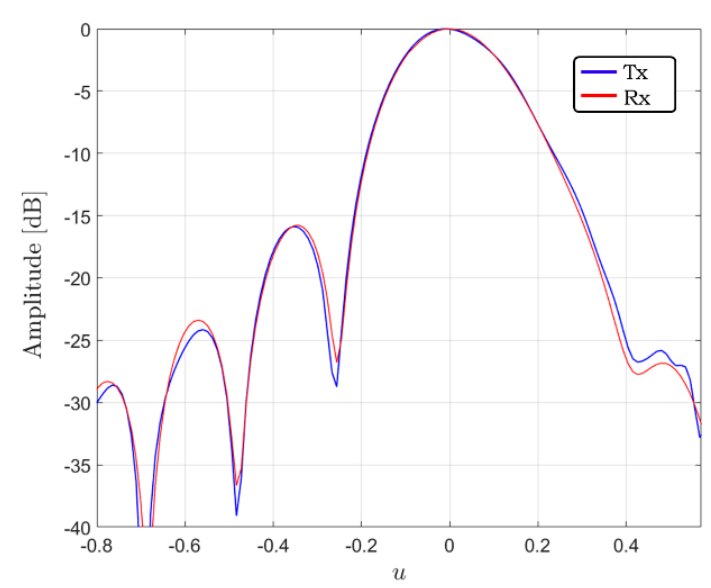

(a)

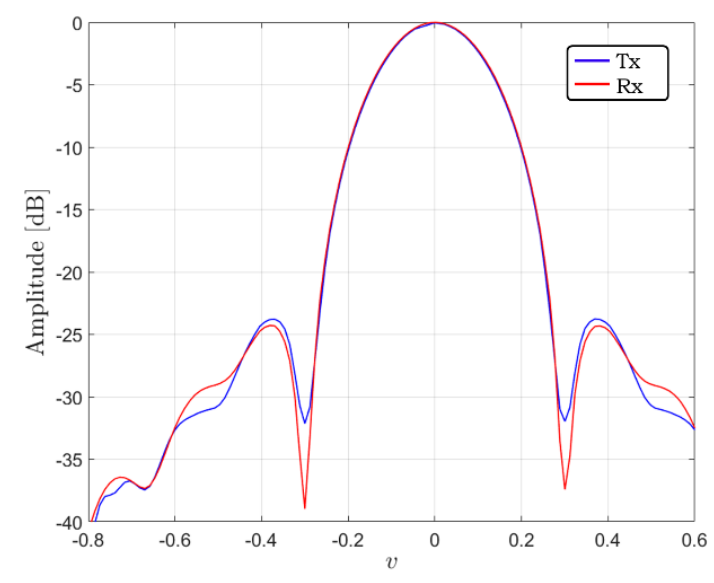

(c)

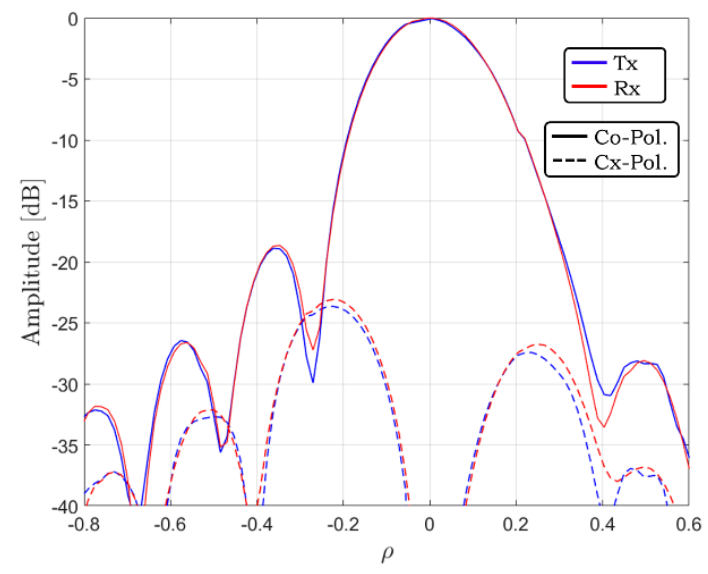

(b)

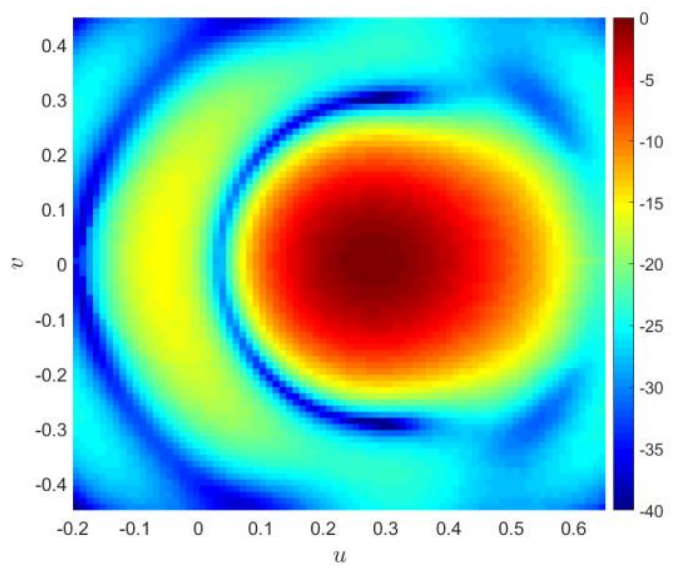

(d)

Figure 6.11: The pattern obtained in Rx of a resonant leaky lens antenna, compared with the pattern evaluated in Tx: (a) $u_{s}$ plane. (b) $\rho_{s}$ plane. (c) $v_{s}$ plane. (d) 3D Co-Pol. pattern in Rx. The elliptical lens is made of silicon $\left(\varepsilon_{r}=11.9\right)$, with $D_{l}=2.78 \mathrm{~mm}$ and $f_{\#}^{l}=1.54\left(\theta_{0}^{l}=19^{\circ}\right)$, illuminated by unitary Co-Pol. and Cx-Pol. plane waves. The feed antenna is x-polarized and operated at $540 \mathrm{GHz}$, shifted from the broadside position with the distance $\vec{d}_{a}=0.375 \mathrm{~mm}$. 


\subsubsection{Hemispherical lens}

In this case of a hemispherical lens, we place a leaky-wave slot below the lens as the receiving antenna that intercepts the fields focalized by the lens. Here we consider a hemispherical silicon $\left(\varepsilon_{r}=11.9\right)$ lens with $D_{h l}=5 \mathrm{~mm}, f_{\#}^{h l}=0.526\left(\theta_{0}^{h l}=56^{\circ}\right), R_{s p h}=2.6 \mathrm{~mm}$, and $L=$ $0.362 R_{s p h}$, illuminated by unitary Co-Pol. and $\mathrm{Cx}-\mathrm{Pol}$. plane waves. A quarter-wavelength matching layer at $480 \mathrm{GHz}$ made of Parylene $\left(\varepsilon_{m}=2.62\right)$ is applied. The feed antenna is ypolarized and operated at $720 \mathrm{GHz}$, shifted from the broadside position with the distance $\vec{d}_{a}=$ $0.14 \mathrm{~mm}$, as depicted in Fig. 6.12.

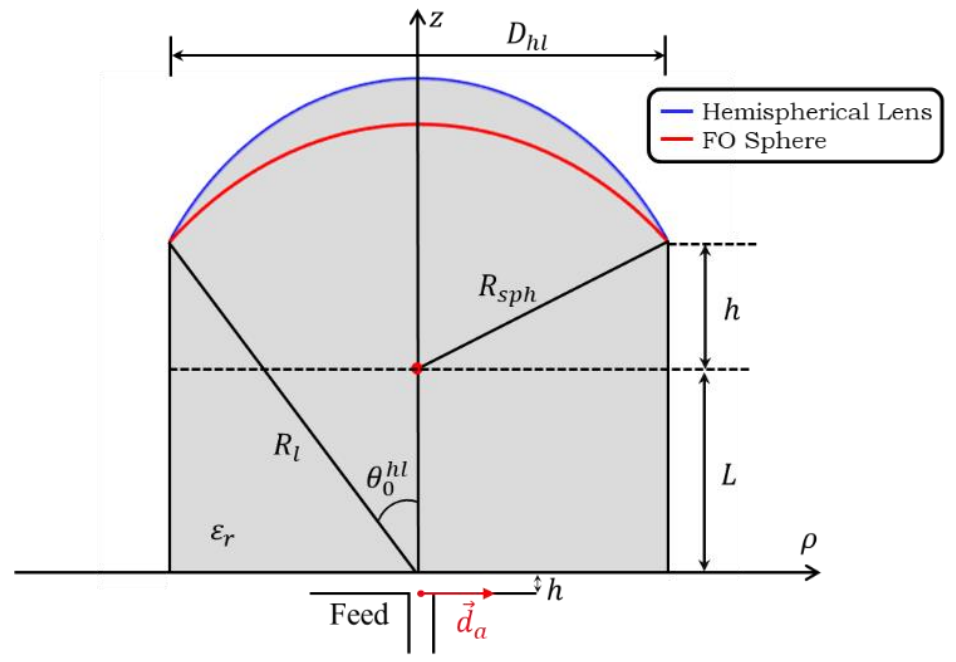

Figure 6.12: Geometry of a leaky hemispherical lens antenna. The hemispherical lens is made of silicon $\left(\varepsilon_{r}=\right.$ $11.9)$, with $D_{h l}=5 \mathrm{~mm}, f_{\#}^{h l}=0.526\left(\theta_{0}^{h l}=56^{\circ}\right), R_{s p h}=2.6 \mathrm{~mm}$, and $L=0.362 R_{s p h}$, illuminated by unitary Co-Pol. and Cx-Pol. plane waves. Here a quarter-wavelength matching layer at $480 \mathrm{GHz}$ made of Parylene $\left(\varepsilon_{m}=\right.$ 2.62 ) is applied. The feed antenna is y-polarized and operated at $720 \mathrm{GHz}$, shifted from the broadside position with the distance $\vec{d}_{a}=0.14 \mathrm{~mm}$.

Fig. 6.13 shows the pattern obtained in Rx, compared with the pattern in Tx. In this case, the antenna far fields are exported from CST and are calculated on the FO sphere by using the antenna propagation approach. The pattern in Tx is the far field transmitted out of the lens, which is evaluated by using the PO in transmission code. Fig. 6.13a to Fig. 6.13c show $u_{s}, \rho_{s}$, and $v_{s}$ planes, respectively; and Fig. $6.13 \mathrm{~d}$ is the 3D Co-pol. pattern in Rx. As it can be seen, the pattern in $\mathrm{Rx}$ is in good agreement with the pattern in $\mathrm{Tx}$, for both the Co-pol. and the Cxpol. components. Moreover, the aperture efficiency, directivity and gain in Rx are validated, as shown in Table. 6.4. 


\begin{tabular}{|c|c|c|c|}
\hline Frequency: 720 GHz & Aperture Efficiency & Directivity [dB] & Gain [dB] \\
\hline $\mathrm{Tx}$ & $39.57 \%$ & 28.37 & 27.50 \\
\hline $\mathrm{Rx}$ & $40.83 \%$ & 28.51 & 27.64 \\
\hline
\end{tabular}

Table 6.4: Performance of the leaky lens antenna evaluated in Rx and compared with the one in Tx.

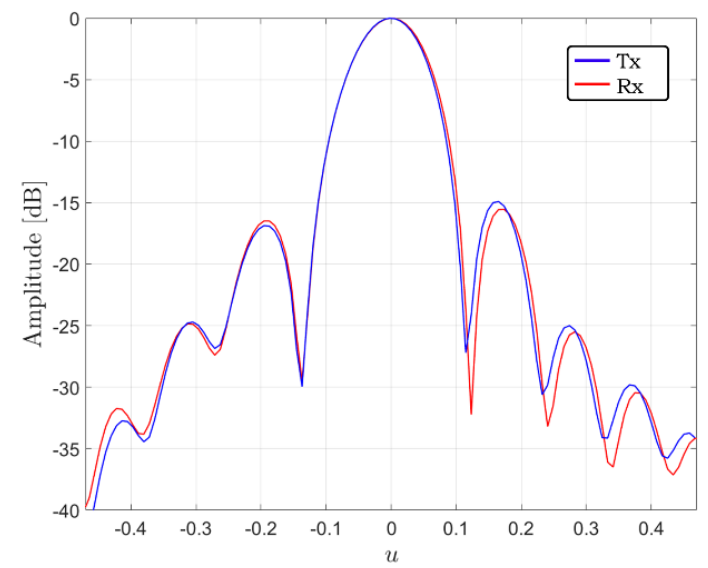

(a)

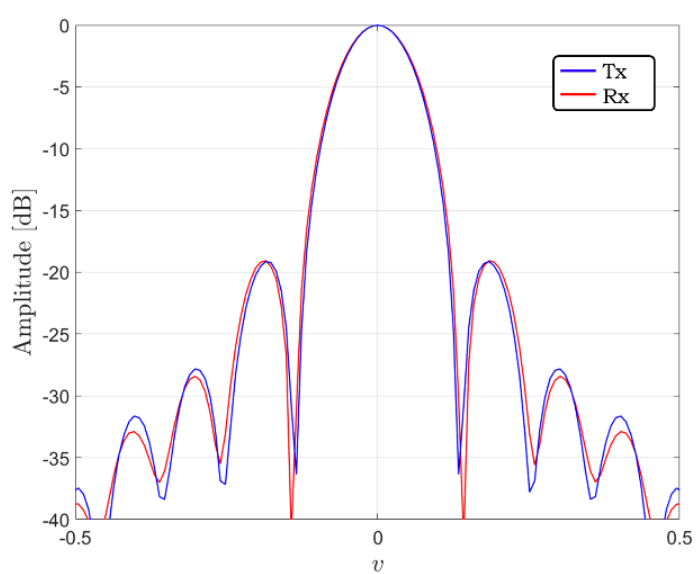

(c)

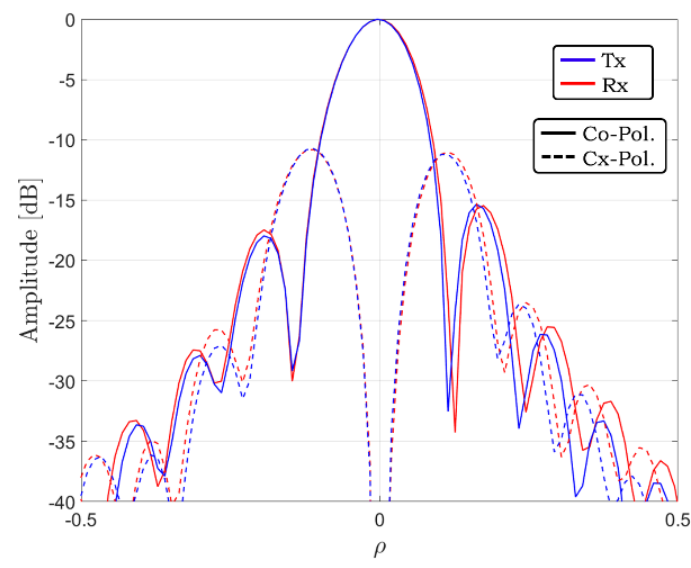

(b)

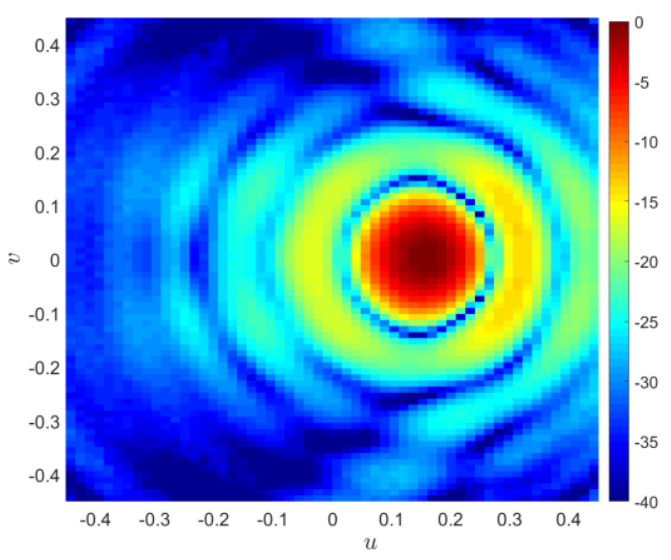

(d)

Figure 6.13: The pattern obtained in Rx of the leaky hemispherical lens antenna, compared with the pattern evaluated in Tx: (a) $u_{s}$ plane. (b) $\rho_{s}$ plane. (c) $v_{s}$ plane. (d) $3 \mathrm{D}$ Co-Pol. pattern in Rx. 


\subsubsection{Hyperbolic lens}

In the case of a hyperbolic lens, we place a leaky lens antenna at its focal plane as the receiving antenna that intercepts the fields focalized by it. Here we consider a hyperbolic plastic $\left(\varepsilon_{r}=\right.$ 2) lens with $D_{h}=0.125 \mathrm{~m}$ and $f_{\#}^{h}=2\left(\theta_{0}^{h}=13^{\circ}\right)$, illuminated by unitary Co-Pol. and CxPol. plane wave. The leaky lens antenna consists of an elliptical silicon $\left(\varepsilon_{r}=11.9\right)$ lens with $D_{l}=1.3 \mathrm{~mm}$ and $f_{\#}^{l}=0.526\left(\theta_{0}^{l}=72^{\circ}\right)$, and a y-polarized leaky-wave slot operated at 720 GHz. It is shifted from the center of the focal plane with the distance $\vec{d}_{a}=20 \mathrm{~mm}$, as depicted in Fig. 6.14.

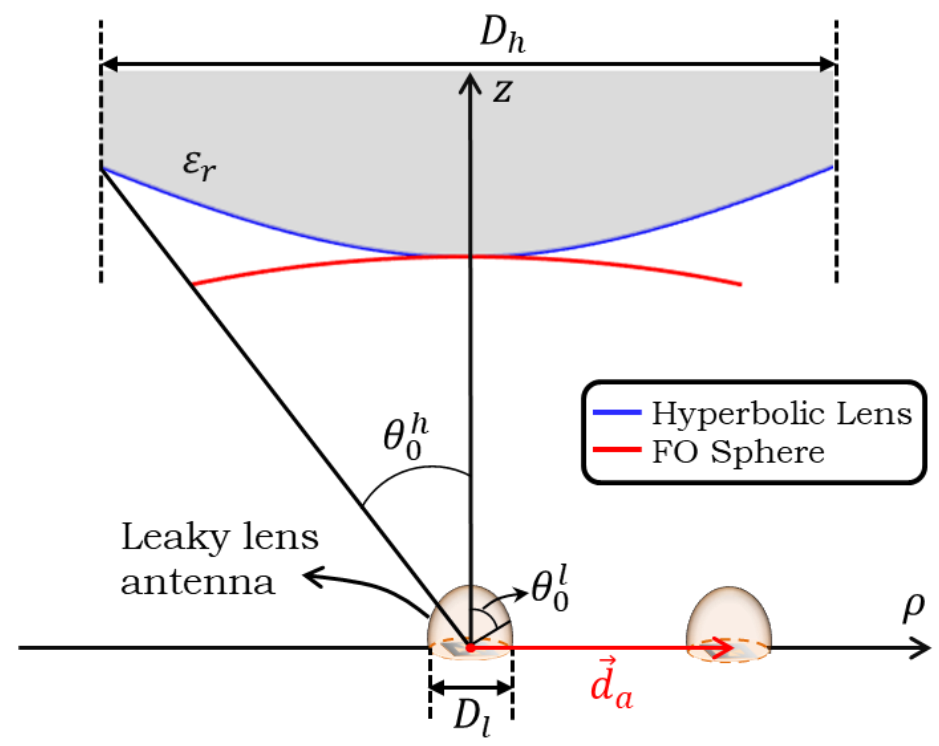

Figure 6.14: Geometry of an antenna-coupled (leaky lens antenna) hyperbolic lens. The hyperbolic plastic $\left(\varepsilon_{r}=\right.$ 2) lens has the diameter of $D_{h}=0.125 \mathrm{~m}$ and the f-number of $f_{\#}^{h}=2\left(\theta_{0}^{h}=13^{\circ}\right)$, illuminated by unitary CoPol. and Cx-Pol. plane waves. At its focal plane, the leaky lens antenna is shifted $\vec{d}_{a}=20 \mathrm{~mm}$ from the center. It consists of an elliptical silicon $\left(\varepsilon_{r}=11.9\right)$ lens with $D_{l}=1.3 \mathrm{~mm}$ and $f_{\#}^{l}=0.526\left(\theta_{0}^{l}=72^{\circ}\right)$, and a ypolarized leaky-wave slot operated at $720 \mathrm{GHz}$.

Fig. 6.15 shows the pattern in Rx, compared with the pattern in Tx. In this case, when calculating the pattern in $\mathrm{Rx}$, the far fields radiated by the leaky lens antenna are evaluated by using the $\mathrm{PO}$ in transmission code. The pattern in $\mathrm{Tx}$ is also obtained using the $\mathrm{PO}$ in transmission code since GRASP cannot evaluate the fields in a dielectric. Fig. 6.15a to Fig. 6.15c show $u_{s}, \rho_{s}$, and $v_{s}$ planes, respectively; and Fig. $6.15 \mathrm{~d}$ is the $3 \mathrm{D}$ Co-pol. pattern in Rx. As it can be seen, the pattern in $\mathrm{Rx}$ is in great agreement with the pattern in Tx, for both the Co-pol. and the Cx-pol. components. Moreover, the aperture efficiency, the directivity and the gain in Rx are validated, as shown in Table. 6.5. 


\begin{tabular}{|c|c|c|c|}
\hline Frequency: $\mathbf{7 2 0} \mathbf{~ G H z}$ & Aperture Efficiency & Directivity [dB] & Gain [dB] \\
\hline $\mathrm{Tx}$ & $13.37 \%$ & 59.85 & 53.76 \\
\hline $\mathrm{Rx}$ & $12.70 \%$ & 59.67 & 53.53 \\
\hline
\end{tabular}

Table 6.5: Performance of the antenna-coupled (leaky lens antenna) hyperbolic lens, evaluated in $\mathrm{Rx}$ and compared with the one in Tx.

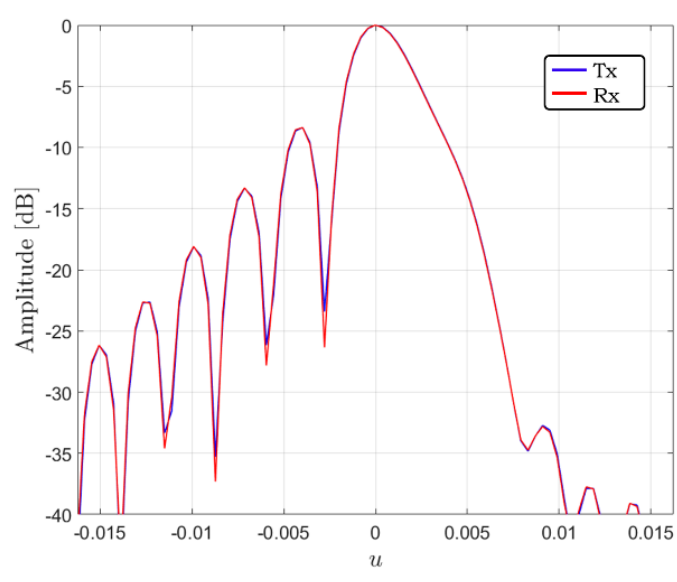

(a)

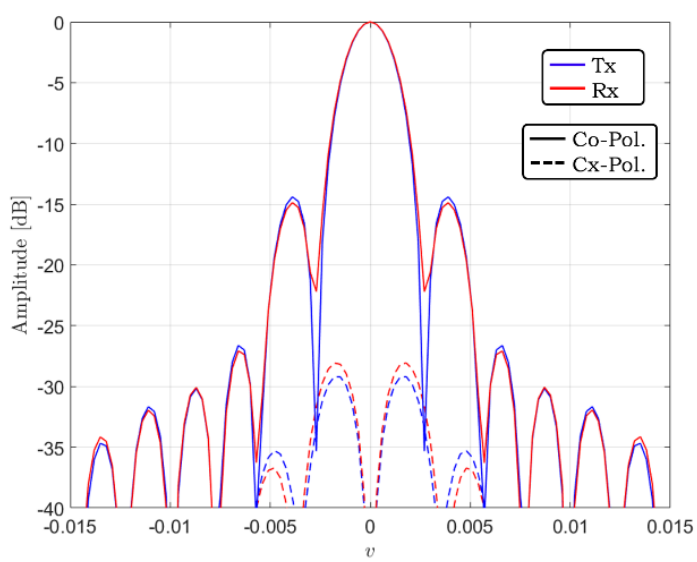

(c)

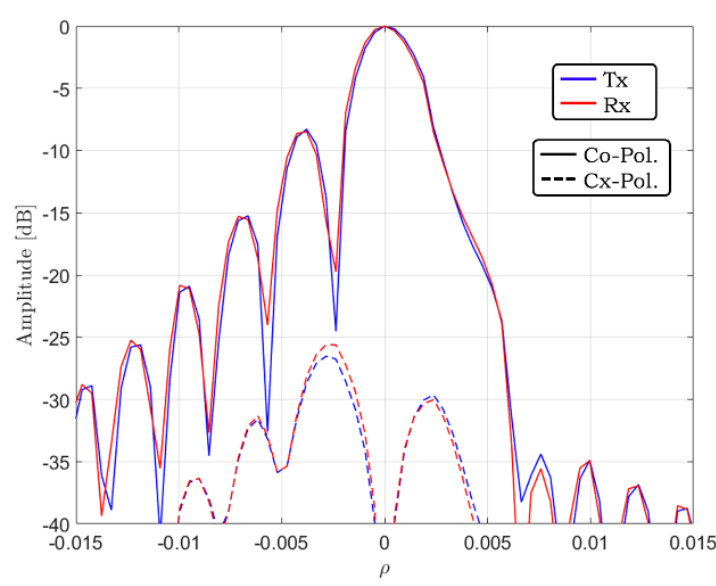

(b)

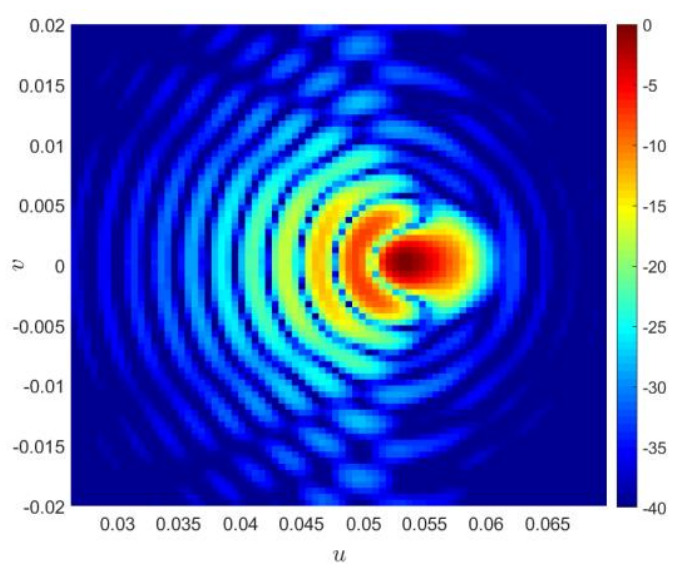

(d)

Figure 6.15: The pattern obtained in Rx of the antenna-coupled (leaky lens antenna) hyperbolic lens, compared with the pattern evaluated in Tx: (a) $u_{s}$ plane. (b) $\rho_{s}$ plane. (c) $v_{s}$ plane. (d) 3D Co-Pol. pattern in Rx. 


\subsubsection{Elliptical Mirror}

For an elliptical mirror, incident fields are emitted by a Huygens source placed at one of its focal planes; while a feed antenna is placed at the other focal plane where the reflected fields are focalized. Here we consider a mirror with the diameter of $D_{m}=0.125 \mathrm{~m}$, the semi-major axis of $a=0.3125 \mathrm{~m}$, and the focal distance of $c=0.0625 \mathrm{~m}$, i.e. the eccentricity is $e=0.2$.

\section{- Case 1: Antenna placed at the upper focal plane}

In this case, the $\mathrm{f}$-number is calculated as $f_{\#}^{m}=2\left(\theta_{0}^{m}=14.4^{\circ}\right)$. The incident fields are generated by unitary Co-Pol. and Cx-Pol. Huygens sources placed at the lower focal plane. A leaky lens antenna is placed at the upper focal plane as the receiving antenna that intercepts the fields focalized by the mirror. It consists of an elliptical silicon $\left(\varepsilon_{r}=11.9\right)$ lens with $D_{l}=$ $1.3 \mathrm{~mm}$ and $f_{\#}^{l}=0.526\left(\theta_{0}^{l}=72^{\circ}\right)$, and a y-polarized leaky-wave slot operated at $720 \mathrm{GHz}$. The leaky lens antenna is shifted from the center of the focal plane with the distance $\vec{d}_{a}=$ $20 \mathrm{~mm}$, as depicted in Fig. 6.16.

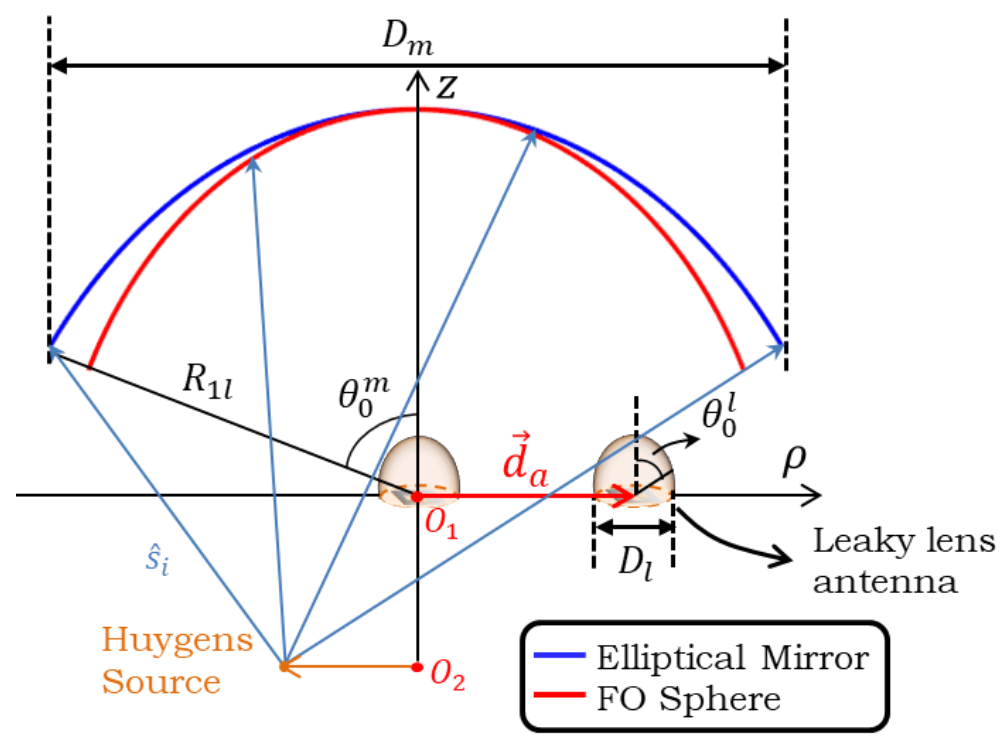

Figure 6.16: Geometry of an antenna-coupled (leaky lens antenna) elliptical mirror. The mirror has the diameter of $D_{m}=0.125 \mathrm{~m}$, the f-number of $f_{\#}^{m}=2\left(\theta_{0}^{m}=14.4^{\circ}\right)$, and the eccentricity of $e=0.2$, illuminated by unitary Co-Pol. and Cx-Pol. Huygens sources placed at the lower focal plane. At its upper focal plane, the leaky lens antenna is shifted $\vec{d}_{a}=20 \mathrm{~mm}$ from the center. It consists of an elliptical silicon $\left(\varepsilon_{r}=11.9\right)$ lens with $D_{l}=$ $1.3 \mathrm{~mm}$ and $f_{\#}^{l}=0.526\left(\theta_{0}^{l}=72^{\circ}\right)$, and a y-polarized leaky-wave slot operated at $720 \mathrm{GHz}$.

Fig. 6.17 shows the pattern obtained in Rx, compared with the pattern in Tx. In this case, when calculating the pattern in $\mathrm{Rx}$, the far fields radiated by the leaky lens antenna are evaluated by using the PO in transmission code. The pattern in Tx is obtained by importing the far-field pattern of the same leaky lens antenna into GRASP. Fig. 6.17a to Fig. 6.17c show $u_{s}$, 
$\rho_{s}$, and $v_{s}$ planes, respectively; and Fig. $6.17 \mathrm{~d}$ is the 3D Co-pol. pattern in Rx. It can be seen that the pattern in Rx is well validated, for both the Co-pol. and the Cx-pol. components. Moreover, the aperture efficiency, the directivity and the gain in Rx are in great agreement with the ones in Tx, as shown in Table. 6.6.

\begin{tabular}{|c|c|c|c|}
\hline Frequency: 720 GHz & Aperture Efficiency & Directivity [dB] & Gain [dB] \\
\hline $\mathrm{Tx}$ & $24.45 \%$ & 58.96 & 53.37 \\
\hline $\mathrm{Rx}$ & $24.23 \%$ & 58.92 & 53.33 \\
\hline
\end{tabular}

Table 6.6: Performance of the antenna-coupled elliptical mirror (leaky lens antenna placed at the upper focal plane), evaluated in $\mathrm{Rx}$ and compared with the one in Tx.

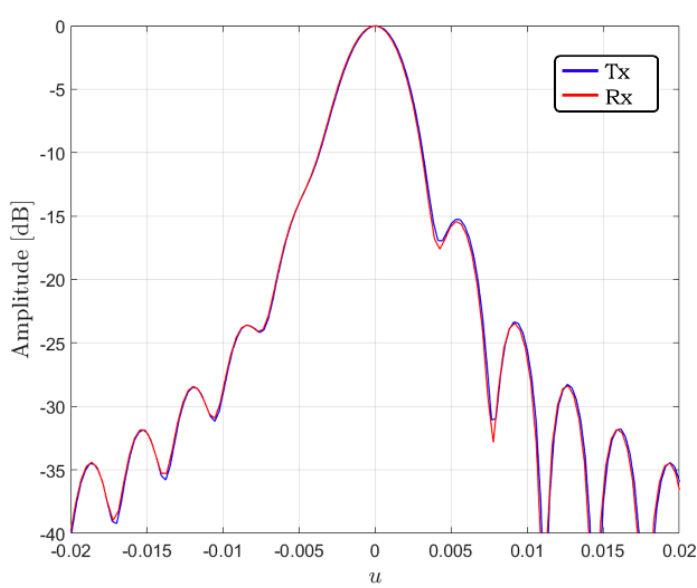

(a)

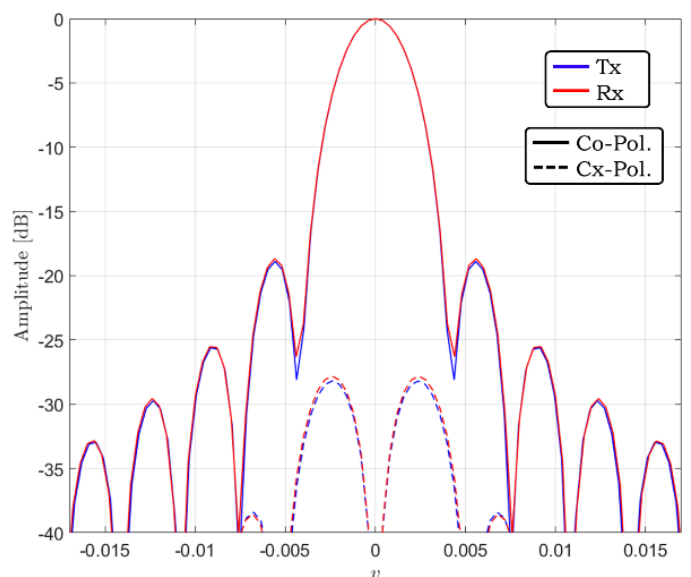

(c)

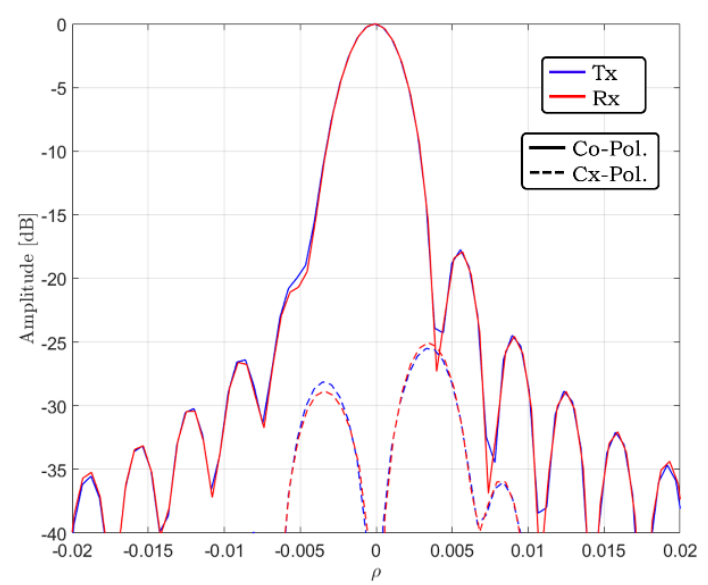

(b)

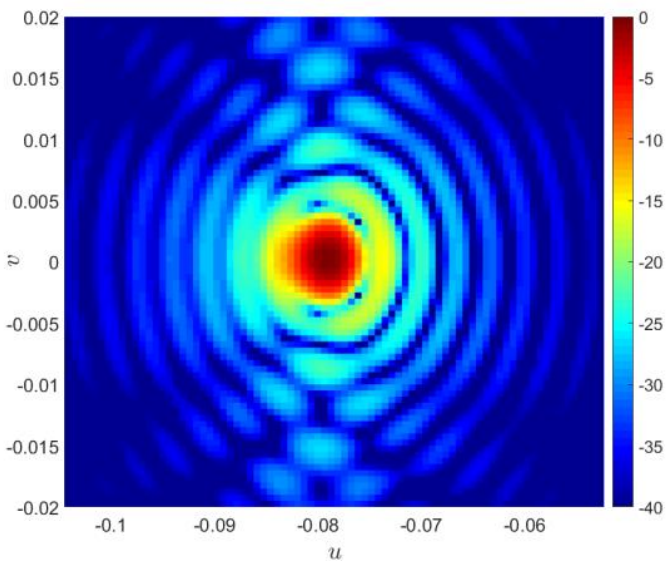

(d)

Figure 6.17: The pattern obtained in Rx of the antenna-coupled elliptical mirror (leaky lens antenna placed at the upper focal plane), compared with the pattern evaluated in Tx: (a) $u_{s}$ plane. (b) $\rho_{s}$ plane. (c) $v_{s}$ plane. (d) 3D CoPol. pattern in Rx. 


\section{- Case 2: Antenna placed at the lower focal plane}

In this case, the f-number is calculated as $f_{\#}^{m}=3\left(\theta_{0}^{m}=9.6^{\circ}\right)$. The incident fields are generated by unitary Co-Pol. and Cx-Pol. Huygens sources placed at the upper focal plane. The same leaky lens antenna described in case 1 is now placed at the lower focal plane as the receiving antenna. It is shifted from the center of the focal plane with the distance $\vec{d}_{a}=$ $33.75 \mathrm{~mm}$, as depicted in Fig. 6.18.

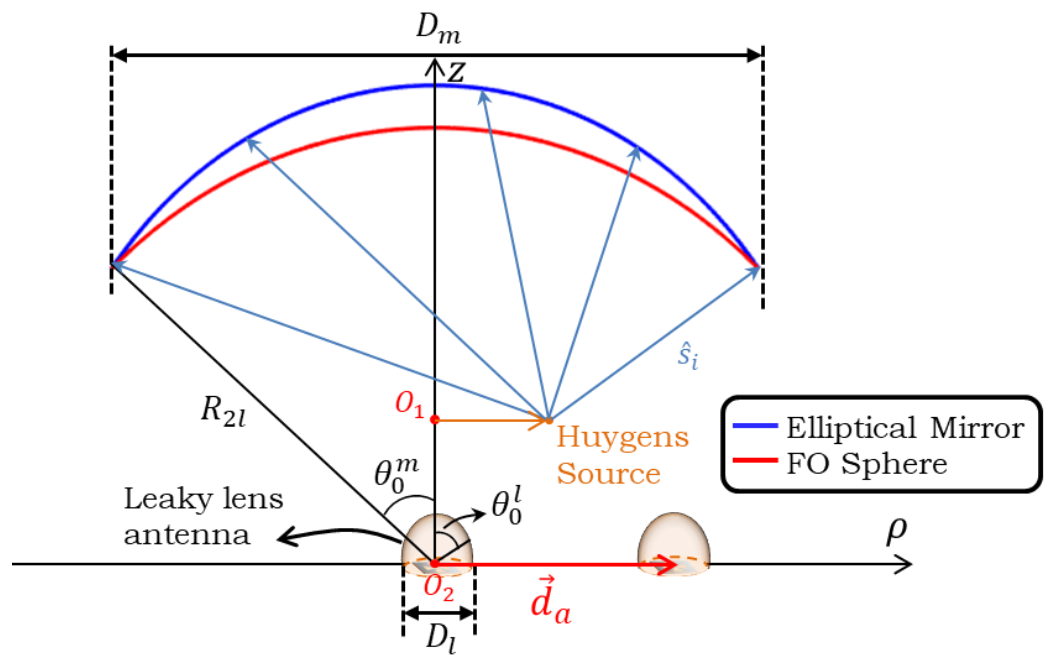

Figure 6.18: Geometry of an antenna-coupled (leaky lens antenna) elliptical mirror. The mirror has the diameter of $D_{m}=0.125 \mathrm{~m}$, the f-number of $f_{\#}^{m}=3\left(\theta_{0}^{m}=9.6^{\circ}\right)$, and the eccentricity of $e=0.2$, illuminated by unitary Co-Pol. and Cx-Pol. Huygens sources placed at the upper focal plane. At its lower focal plane, the leaky lens antenna is shifted $\vec{d}_{a}=33.75 \mathrm{~mm}$ from the center. It consists of an elliptical silicon $\left(\varepsilon_{r}=11.9\right)$ lens with $D_{l}=$ $1.3 \mathrm{~mm}$ and $f_{\#}^{l}=0.526\left(\theta_{0}^{l}=72^{\circ}\right)$, and a y-polarized leaky-wave slot operated at $720 \mathrm{GHz}$.

Fig. 6.19 shows the pattern obtained in Rx, compared with the pattern in Tx. Fig. 6.19a to Fig. 6.19c show $u_{s}, \rho_{s}$, and $v_{s}$ planes, respectively; and Fig. 6.19d is the 3D Co-pol. pattern in Rx. It can be seen that the pattern in Rx is well validated. Moreover, the aperture efficiency, the directivity and the gain in Rx are also validated, as shown in Table. 6.7.

\begin{tabular}{|c|c|c|c|}
\hline Frequency: 720 GHz & Aperture Efficiency & Directivity [dB] & Gain [dB] \\
\hline $\mathrm{Tx}$ & $12.16 \%$ & 58.68 & 50.33 \\
\hline $\mathrm{Rx}$ & $11.98 \%$ & 58.62 & 50.27 \\
\hline
\end{tabular}

Table 6.7: Performance of the antenna-coupled elliptical mirror (leaky lens antenna placed at the lower focal plane), evaluated in Rx and compared with the one in Tx. 


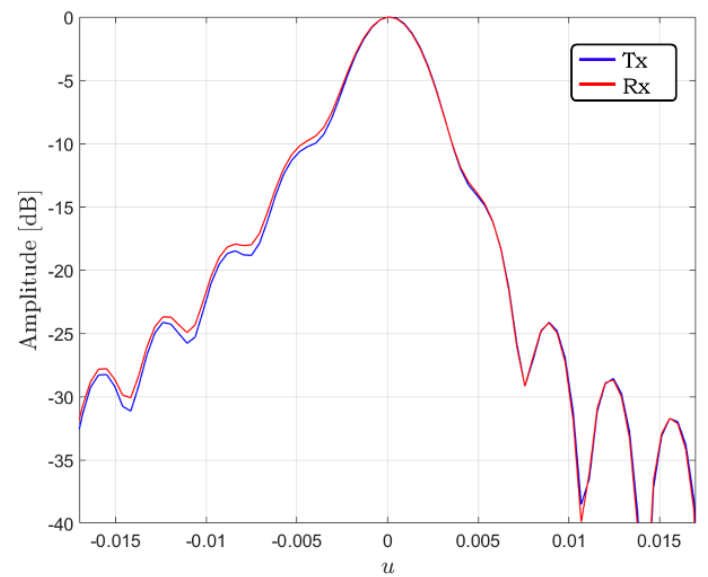

(a)

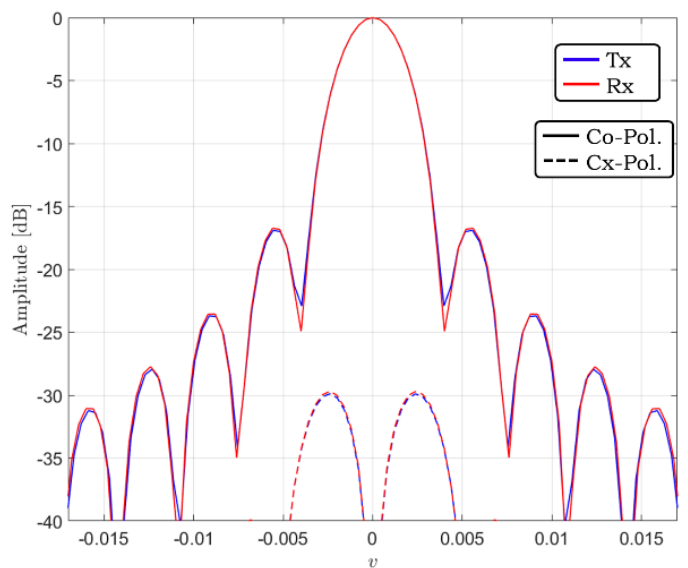

(c)

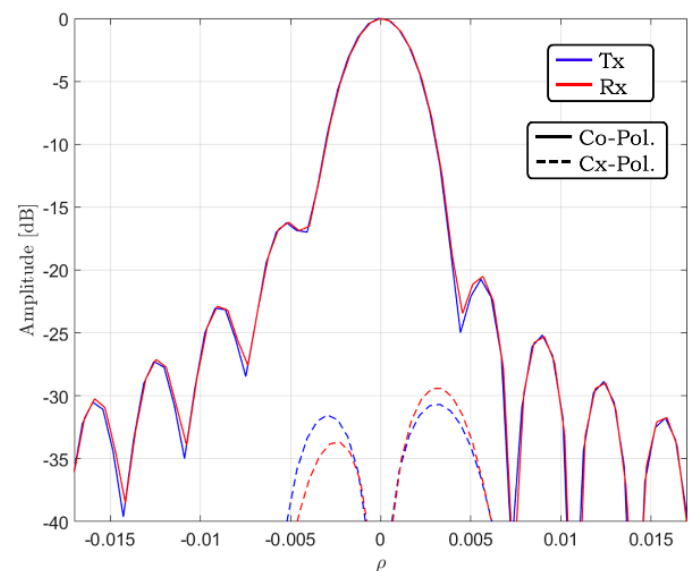

(b)

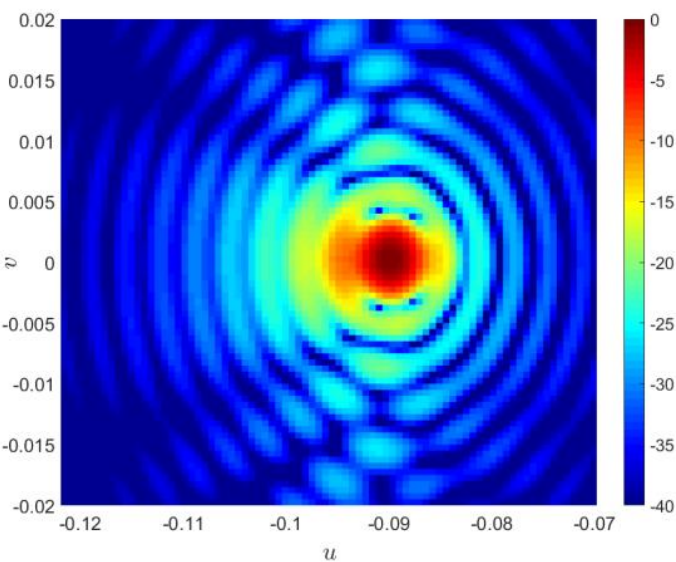

(d)

Figure 6.19: The pattern obtained in Rx of the antenna-coupled elliptical mirror (leaky lens antenna placed at the lower focal plane), compared with the pattern evaluated in Tx: (a) $u_{s}$ plane. (b) $\rho_{s}$ plane. (c) $v_{s}$ plane. (d) 3D CoPol. pattern in Rx. 


\section{Chapter 7: Designed GUI Tool}

FO method is a powerful tool to represent the focalized field by a PWS at the focal plane of a QO component in reception. By using the PWS, one can analyze the performance of detectorcoupled QO systems. However to our knowledge, no universal tools are developed to implement this FO method for variety of QO components in reception. There are a few codes implementing the FO method [7, 15, 16], but they are limited in applicability. First, existing codes do not include variety of QO components. They mainly focus on analyzing parabolic reflectors and elliptical lenses. Moreover, they are not built with user-friendly interfaces, which means one cannot operate them easily. Accordingly, in this thesis, a MATLAB based GUI tool is built to analyze the antenna-coupled QO systems in reception by using the FO method. The tool includes five canonical QO components to improve design possibilities. Also, the user interface is informative so users do not need to know the details of FO methodology. In addition, the tool takes into account the presence of a matching layer, which is an essential element for designing dielectric lenses.

In this chapter, we will show the interface of the tool and explain how it implements the methods described from chapter 2 to chapter 6 to analyze antenna-coupled QO systems in reception. The GUI tool is divided into two sub-GUIs and the interface of the main GUI is shown in Fig. 7.1. This main GUI is introduced from section 7.1 to 7.4. First in section 7.1, we briefly introduce how it defines the main QO components and implements the ray tracing technique. Next in section 7.2, we discuss how it implements the coherent method. And then in section 7.3, we explain how the GUI calculates GO fields on a FO sphere. Finally, in section 7.4, numerical examples of GO fields and focal plane fields evaluated by the GUI are shown. In section 7.5, the second GUI is introduced. It is used to analyze the performance of antennacoupled QO systems. Conclusion is in section 7.6. 


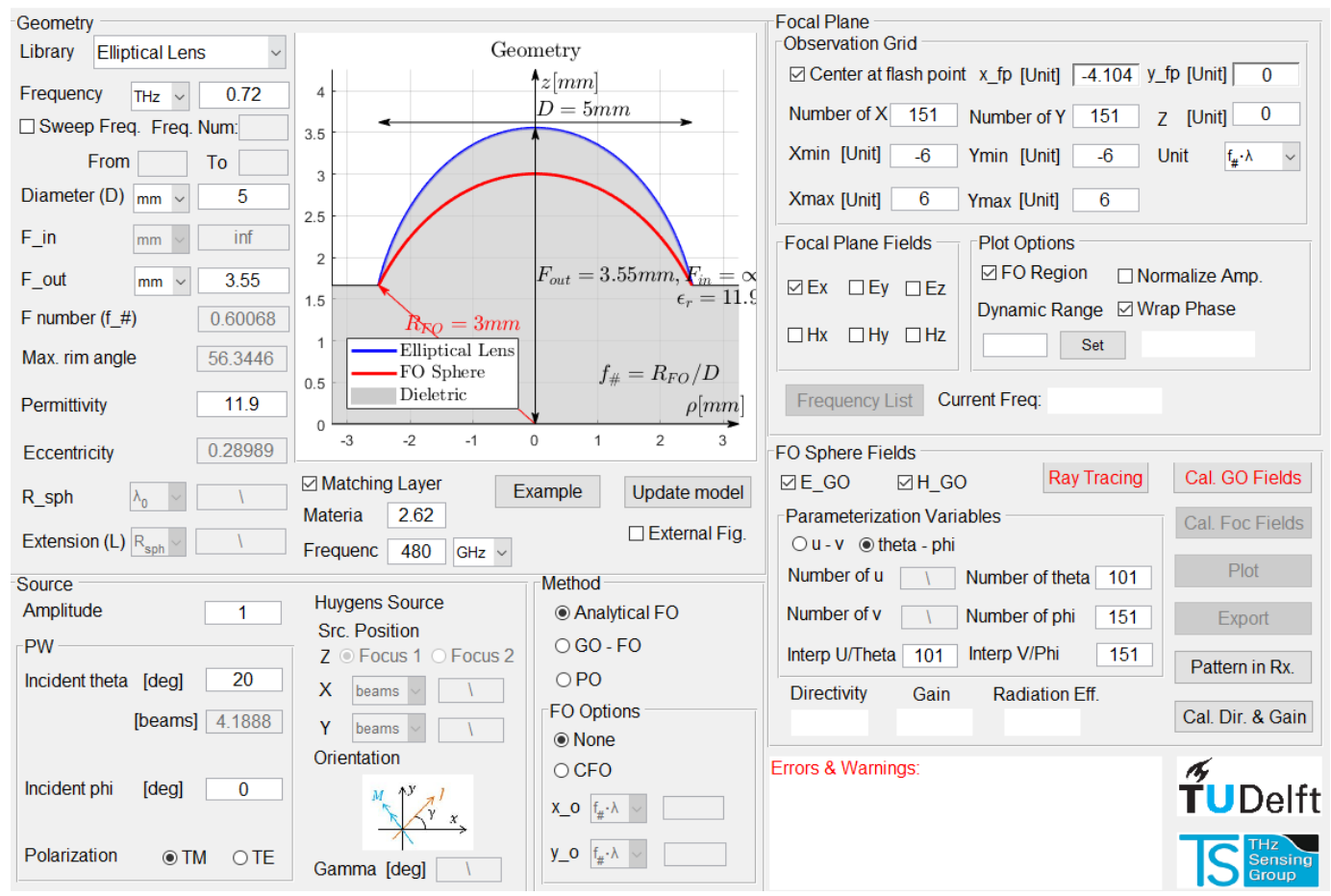

Figure 7.1: Layout of the main GUI tool.

\subsection{Main QO components and ray tracing}

\section{- Define a QO surface}

In chapter 2, five widely used QO components are parameterized, namely parabolic reflectors, elliptical lenses, hemispherical lenses, hyperbolic lenses, and elliptical mirrors. And in this section, we mainly discuss how to define them in the main GUI. Fig. 7.2 shows the interface used to define QO components. One can choose a QO surface from the "Library" and define it by inputting necessary parameters. As it can be seen in the figure, here we define an elliptical silicon $\left(\varepsilon_{r}=11.9\right)$ lens with a matching layer. The corresponding $2 \mathrm{D}$ geometry is plotted in the inset to visualize the surface. 


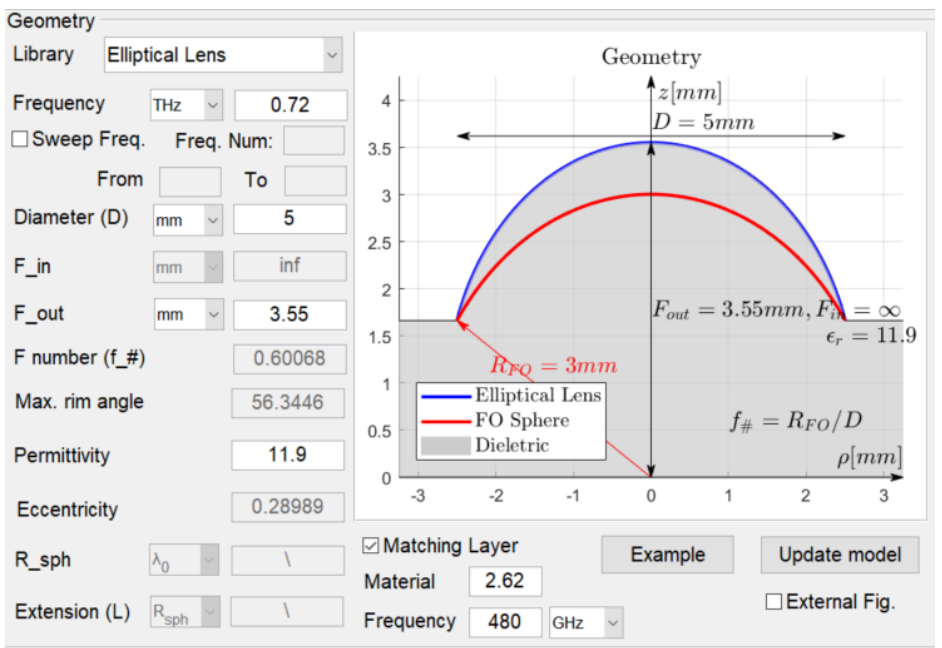

Figure 7.2: Interface used to define QO components

\section{- Ray tracing}

An in-house ray tracing code is developed to describe and visualize reception scenarios for all QO components. Here we take the preceding elliptical silicon lens as an example. The lens has the diameter of $D_{l}=5 \mathrm{~mm}$ and the f-number of $f_{\#}^{l}=0.6$. A quarter-wavelength matching layer at $480 \mathrm{GHz}$ made of Parylene $\left(\varepsilon_{m}=2.62\right)$ is applied.

To start a ray tracing, one needs to define incident fields: A unitary TM polarized plane wave operated at $720 \mathrm{GHz}$ with the skew angle of $\theta_{s}=20^{\circ}, \phi_{s}=0^{\circ}$ is defined, as shown in Fig. 7.3a. Meanwhile, an observation plane should be chosen. And then the ray tracing of this scenario is plotted in Fig. 7.3b. It can be seen in the figure that the ray tracing includes six basic components: The incident and transmitted rays follow Snell's law described in Eq. (2.30). The FO sphere is chosen as large as possible and is defined in section 3.2.2. The lens surface (section 2.1.3) is half transparent and the dielectric slab is from the edge of the surface to the focal plane. Moreover, the observation plane is a $4 \mathrm{~mm}$ by $4 \mathrm{~mm}$ square centered at the focal plane. In this work, it is approximated that the thin matching layer does not change the ray path. Therefore, the layer is not shown. By using this ray tracing tool, one is able to preliminarily analyze QO systems by minimizing phase aberrations associated to different path lengths. 


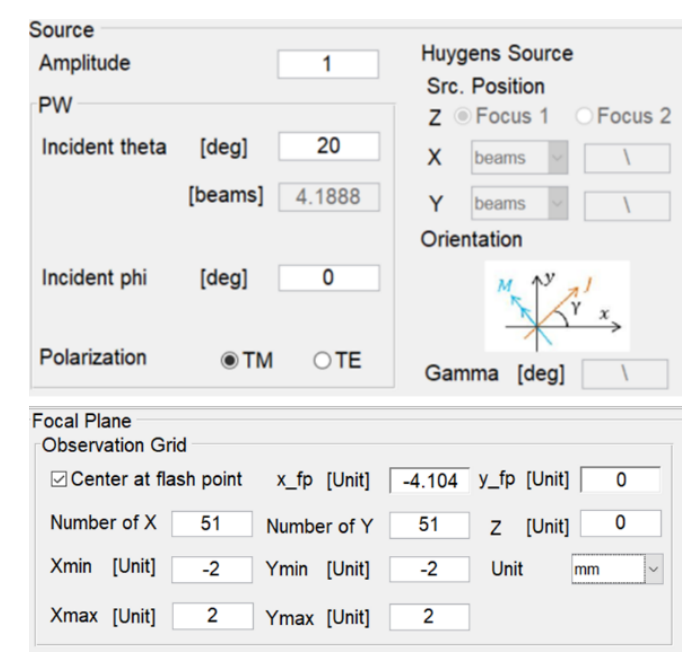

(a)

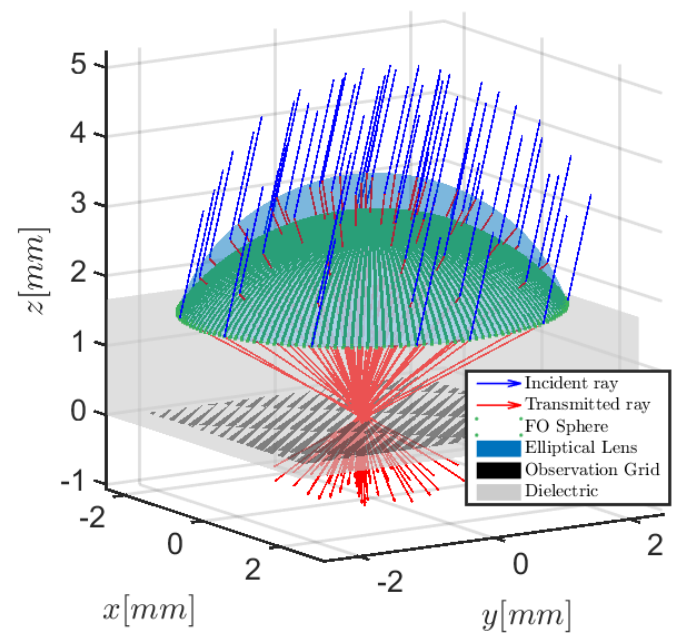

(b)

Figure 7.3: Ray tracing tool: (a) Interface used to set up ray tracing. (b) Ray tracing plot for the elliptical silicon lens illuminated by a unitary TM polarized plane wave with the skew angle of $\theta_{s}=20^{\circ}, \phi_{s}=0^{\circ}$.

\subsection{Coherent FO}

In the section of the FO integral, section 3.2, we mentioned that the focal plane electric field can be evaluated by using both the GO electric and magnetic fields, $\vec{E}_{G O}$ and $\vec{H}_{G O}$, as described in Eq. (3.9); or using only the GO electric field, $\vec{E}_{G O}$, as describe in Eq. (3.11), which depends on the incident skew angle. When the skew angle is relatively small, e.g. $\theta_{s} \leq 11^{\circ}$, one can assume $\vec{E}_{G O}$ and $\vec{H}_{G O}$ are tangent to the FO sphere. In this case, one can use either $\vec{E}_{G O}$ or $\vec{H}_{G O}$ to evaluate the focal plane fields. While for large skew angles, the tangential condition is not applicable. Therefore, one needs both $\vec{E}_{G O}$ and $\vec{H}_{G O}$. This is the same case when evaluating the coherent FO spectra.

In the tool, there are two options to calculate coherent FO spectra. In the first option, the tool uses both GO electric and magnetic fields, $\vec{E}_{G O}$ and $\vec{H}_{G O}$. While in the second option, only one of the GO fields is used, either $\vec{E}_{G O}$ or $\vec{H}_{G O}$. In the following, we will explain what is implemented in the tool for these two options.

\section{- Option 1: Using both $\vec{E}_{G O}$ and $\vec{H}_{G O}$}

In this case, the focal plane electric field can be expressed as follows:

$$
\begin{aligned}
\vec{e}_{f}^{E \& H}\left(\vec{\rho}_{f}\right)=\frac{j k e^{-j k R_{F O}} e^{-j k \frac{\rho_{f}^{2}}{2 R_{F O}}}}{4 \pi R_{F O}} . \\
\int_{S_{F O}}\left\{\left[\vec{E}_{G O}-\left(\hat{n} \cdot \vec{E}_{G O}\right) \hat{n}\right]-\zeta\left(\hat{n} \times \vec{H}_{G O}\right)\right\} e^{j k \vec{\rho}_{f} \cdot \hat{r}^{\prime}} d \vec{r}^{\prime}
\end{aligned}
$$


where $\vec{\rho}_{f}$ is a point on the observation plane, $R_{F O}$ is the radius of a FO sphere, $\hat{n}$ is the normal vector of the FO sphere, and $\vec{r}^{\prime}$ is a point on the FO sphere. Moreover, the magnetic field can be calculated as:

$$
\begin{aligned}
\vec{h}_{f}^{E \& H}\left(\vec{\rho}_{f}\right)=\frac{j k e^{-j k R_{F O}} e^{-j k \frac{\rho_{f}^{2}}{2 R_{F O}}}}{4 \pi R_{F O}} . \\
\qquad \int_{S_{F O}}\left\{\left[\vec{H}_{G O}-\left(\hat{n} \cdot \vec{H}_{G O}\right) \hat{n}\right]+\frac{1}{\zeta}\left(\hat{n} \times \vec{E}_{G O}\right)\right\} e^{j k \vec{\rho}_{f} \cdot \hat{r}^{\prime}} d \vec{r}^{\prime}
\end{aligned}
$$

In Eq. (7.1) and (7.2), since we do not apply any approximation on GO fields, the focal plane fields are calculated accurately within the FO applicability region. Furthermore, the electric field $\vec{e}_{f}^{E \& H}$ can be expressed by its Cartesian spectral representation, as discussed in section 3.2.3. In this representation, the electric spectral function $\vec{E}_{F O}\left(k_{x}, k_{y}\right)$ can be expressed as:

$$
\vec{E}_{F O}^{E \& H}\left(k_{x}, k_{y}\right)=\frac{j \pi R_{F O} e^{-j k R_{F O}}}{k_{z}}\left\{\left[\vec{E}_{G O}-\left(\hat{n} \cdot \vec{E}_{G O}\right) \hat{n}\right]-\zeta\left(\hat{n} \times \vec{H}_{G O}\right)\right\} \operatorname{circ}\left(k_{\rho}, k_{\rho 0}\right)
$$

where $k_{\rho}=k \sin \theta$ and $k_{z}=\sqrt{k^{2}-k_{\rho}^{2}}$. While for the magnetic one:

$$
\vec{H}_{F O}^{E \& H}\left(k_{x}, k_{y}\right)=\frac{j \pi R_{F O} e^{-j k R_{F O}}}{k_{z}}\left\{\left[\vec{H}_{G O}-\left(\hat{n} \cdot \vec{H}_{G O}\right) \hat{n}\right]+\frac{1}{\zeta}\left(\hat{n} \times \vec{E}_{G O}\right)\right\} \operatorname{circ}\left(k_{\rho}, k_{\rho 0}\right)
$$

Therefore, the linearized coherent FO spectrum described in section 3.3.2 can be evaluated by using $\vec{E}_{F O}^{E \& H}\left(k_{x}, k_{y}\right)$ as:

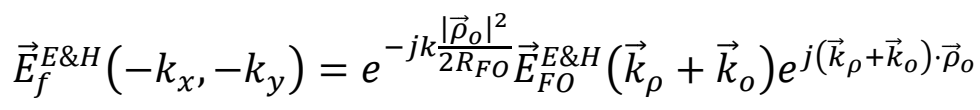

where $\vec{\rho}_{o}$ is the point where we introduce the linearization and $\vec{k}_{o}=\frac{k}{R_{F O}} \vec{\rho}_{o}$. Moreover, the magnetic spectrum is:

$$
\vec{H}_{f}^{E \& H}\left(-k_{x},-k_{y}\right)=e^{-j k \frac{\left|\vec{\rho}_{o}\right|^{2}}{2 R_{F O}}} \vec{H}_{F O}^{E \& H}\left(\vec{k}_{\rho}+\vec{k}_{o}\right) e^{j\left(\vec{k}_{\rho}+\vec{k}_{o}\right) \cdot \vec{\rho}_{o}}
$$

These electric and magnetic spectra can be exported by the tool and used in spectral techniques such as equivalent Floquet circuits. To validate these spectra, we have mentioned in chapter 5 that we indirectly validate the focal plane fields calculated by using these spectra:

$$
\left\{\begin{array}{l}
\vec{e}_{f}^{E \& H}\left(\vec{\rho}^{\prime}\right)=\frac{1}{4 \pi^{2}} \int_{-\infty}^{\infty} \int_{-\infty}^{\infty} \vec{E}_{f}^{E \& H}\left(-k_{x},-k_{y}\right) e^{j k_{x} x^{\prime}} e^{j k_{y} y^{\prime}} d k_{x} d k_{y} \\
\vec{h}_{f}^{E \& H}\left(\vec{\rho}^{\prime}\right)=\frac{1}{4 \pi^{2}} \int_{-\infty}^{\infty} \int_{-\infty}^{\infty} \vec{H}_{f}^{E \& H}\left(-k_{x},-k_{y}\right) e^{j k_{x} x^{\prime}} e^{j k_{y} y^{\prime}} d k_{x} d k_{y}
\end{array}\right.
$$

where $\vec{\rho}^{\prime}$ is the point surrounding around $\vec{\rho}_{o}$. 


\section{- Option 2: Using either $\vec{E}_{G O}$ or $\vec{H}_{G O}$}

In this case, we assume the GO magnetic field is orthogonal to the electric field with respect to the normal to the FO sphere, $\hat{n}$, as follows:

$$
\vec{H}_{G O}=\frac{1}{\zeta} \hat{n} \times \vec{E}_{G O}
$$

By using Eq. (7.8), the focal plane fields described in Eq. (7.1) and (7.2) can be simplified as:

$$
\left\{\begin{array}{l}
\vec{e}_{f}^{E / H}\left(\vec{\rho}_{f}\right) \simeq \frac{j k e^{-j k R_{F O}} e^{-j k \frac{\rho_{f}^{2}}{2 R_{F O}}}}{4 \pi R_{F O}} \int_{S_{F O}} 2 \vec{E}_{G O}(\theta, \phi) e^{j k \vec{\rho}_{f} \cdot \hat{r}^{\prime}} R_{F O}^{2} \sin \theta d \theta d \phi \\
\vec{h}_{f}^{E / H}\left(\vec{\rho}_{f}\right) \simeq \frac{j k e^{-j k R_{F O}} e^{-j k \frac{\rho_{f}^{2}}{2 R_{F O}}}}{4 \pi R_{F O}} \int_{S_{F O}} 2 \vec{H}_{G O}(\theta, \phi) e^{j k \vec{\rho}_{f} \cdot \hat{r}^{\prime}} R_{F O}^{2} \sin \theta d \theta d \phi
\end{array}\right.
$$

Moreover, the spectral functions $\vec{E}_{F O}\left(k_{x}, k_{y}\right)$ and $\vec{H}_{F O}\left(k_{x}, k_{y}\right)$ can be approximated as:

$$
\left\{\begin{array}{l}
\vec{E}_{F O}^{E / H}\left(k_{x}, k_{y}\right) \simeq \frac{j 2 \pi R_{F O} e^{-j k R_{F O}}}{k_{z}} \vec{E}_{G O}\left(k_{x}, k_{y}\right) \operatorname{circ}\left(k_{\rho}, k_{\rho 0}\right) \\
\vec{H}_{F O}^{E / H}\left(k_{x}, k_{y}\right) \simeq \frac{j 2 \pi R_{F O} e^{-j k R_{F O}}}{k_{z}} H_{G O}\left(k_{x}, k_{y}\right) \operatorname{circ}\left(k_{\rho}, k_{\rho 0}\right)
\end{array}\right.
$$

Furthermore, the linearized coherent FO spectra can be expressed as:

$$
\left\{\begin{array}{l}
\vec{E}_{f}^{E / H}\left(-k_{x},-k_{y}\right)=e^{-j k \frac{\left|\vec{\rho}_{o}\right|^{2}}{2 F_{F O}}} \vec{E}_{F O}^{E / H}\left(\vec{k}_{\rho}+\vec{k}_{o}\right) e^{j\left(\vec{k}_{\rho}+\vec{k}_{o}\right) \cdot \vec{\rho}_{o}} \\
\vec{H}_{f}^{E / H}\left(-k_{x},-k_{y}\right)=e^{-j k \frac{\left|\vec{\rho}_{o}\right|^{2}}{2 R_{F O}}} \vec{H}_{F O}^{E / H}\left(\vec{k}_{\rho}+\vec{k}_{o}\right) e^{j\left(\vec{k}_{\rho}+\vec{k}_{o}\right) \cdot \vec{\rho}_{o}}
\end{array}\right.
$$

Similar to the option 1 , these spectra can be exported by the tool and are validated by validating their corresponding focal plane fields:

$$
\left\{\begin{array}{l}
\vec{e}_{f}^{E / H}\left(\vec{\rho}^{\prime}\right)=\frac{1}{4 \pi^{2}} \int_{-\infty}^{\infty} \int_{-\infty}^{\infty} \vec{E}_{f}^{E / H}\left(-k_{x},-k_{y}\right) e^{j k_{x} x^{\prime}} e^{j k_{y} y^{\prime}} d k_{x} d k_{y} \\
\vec{h}_{f}^{E / H}\left(\vec{\rho}^{\prime}\right)=\frac{1}{4 \pi^{2}} \int_{-\infty}^{\infty} \int_{-\infty}^{\infty} \vec{H}_{f}^{E / H}\left(-k_{x},-k_{y}\right) e^{j k_{x} x^{\prime}} e^{j k_{y} y^{\prime}} d k_{x} d k_{y}
\end{array}\right.
$$




\subsection{Calculation of GO fields}

The coherent FO spectra are related to the GO fields and we have introduced GO ray fields and analytical GO fields in chapter 4. In this section, we will discuss how the GUI calculates these GO fields.

\section{- Analytical GO fields}

When the incident skew angle $\theta_{s}$ is less than $11^{\circ}$, the GO electric field on a FO sphere can be expressed as:

$$
\vec{E}_{G O}\left(\theta_{S}, \phi_{s}\right) \simeq \vec{E}_{G O}\left(\theta_{s}=0\right) \mathrm{e}^{-j \vec{k}_{\rho} \cdot \vec{\rho}_{f p}} e^{-j \Phi_{c o m a}}\left(e^{-j \Phi_{c o m p}}\right)
$$

where $e^{-j \Phi_{c o m p}}$ is the compensation phase term for the case of elliptical mirrors. Since the skew angle is small, one can use the relation described in Eq. (7.8) to calculate the GO magnetic field $\vec{H}_{G O}\left(\theta_{s}, \phi_{s}\right)$.

\section{- GO ray fields}

When the skew angle is $\theta_{s}>11^{\circ}$, analytical GO fields are not applicable. In such a case, the tool calculates the GO ray fields. The GO electric field can be evaluated by using the spreading factor $S_{\text {read }}(\theta)$ and the distance along the ray path $s(\theta, \phi)$ as:

$$
\vec{E}_{G O}\left(\theta^{\prime}, \phi^{\prime}\right)=\vec{E}_{r / t}(\theta, \phi) \cdot S_{\text {pread }}(\theta) \cdot e^{-j k s(\theta, \phi)}
$$

where $\vec{E}_{r / t}$ is the reflected or transmitted field discussed in section 2.2.2.1, and $\left(\theta^{\prime}, \phi^{\prime}\right)$ are non-uniform parameterization variables in GO propagation which are shown in Fig. 7.4 for a reflection case. The explicit explanation of $\left(\theta^{\prime}, \phi^{\prime}\right)$ is described in Appendix E.1. Moreover, the GO magnetic field is related to $\vec{E}_{G O}$ as:

$$
\vec{H}_{G O}\left(\theta^{\prime}, \phi^{\prime}\right)=\frac{1}{\eta} \hat{s}_{r / t} \times \vec{E}_{G O}\left(\theta^{\prime}, \phi^{\prime}\right)
$$

where $\hat{s}_{r / t}$ is the reflected or transmitted propagation unit vector. It is worth noting that the GO fields, $\vec{E}_{G O}\left(\theta^{\prime}, \phi^{\prime}\right)$ and $\vec{H}_{G O}\left(\theta^{\prime}, \phi^{\prime}\right)$, are non-uniformly distributed on a FO sphere. Therefore, one should numerically interpolate the non-uniform fields $\overrightarrow{E / H}_{G O}\left(\theta^{\prime}, \phi^{\prime}\right)$ to the uniform ones

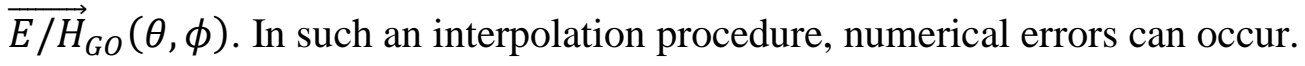




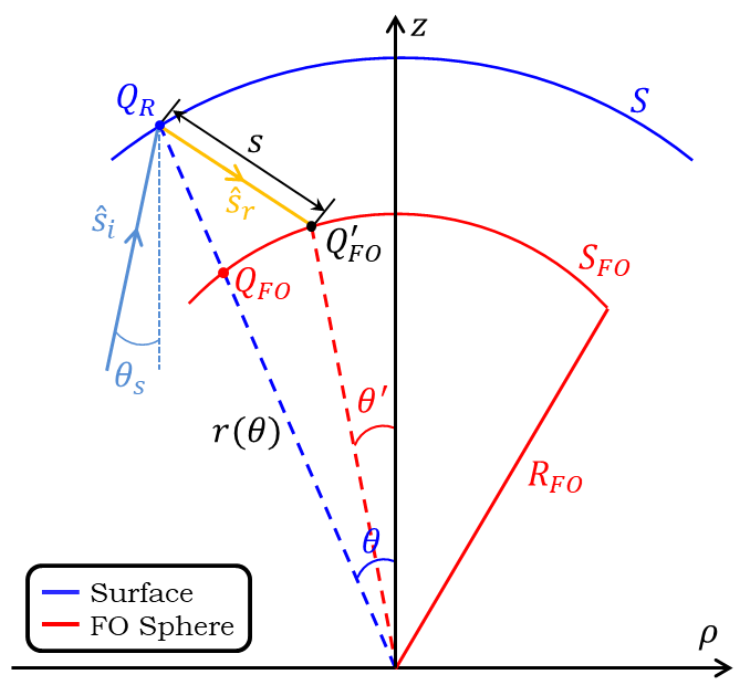

Figure 7.4: GO propagation in a reflection problem.

\subsection{Numerical examples of GO fields and focal plane fields}

In this section, we will first introduce some configurations in the main GUI, and then show some numerical examples of GO fields and focal plane fields calculated by the GUI.

\subsubsection{Configurations in the GUI}

Fig. 7.5 shows the configurations set in the GUI for calculating GO and focal plane fields. In Fig. 7.5a, there are three approaches to calculate focal plane fields. "PO" is used as validation. It calculates focal plane fields by using the PO radiation integral.

In the FO method, "Analytical FO" means that GO fields are calculated analytically by using Eq. (7.13). While "GO-FO" uses the GO ray fields described in Eq. (7.14). By using the GO fields, one can calculate the focal plane fields in the presence of the quadratic phase term, i.e. "None" option. In this option, the GUI implements Eq. (7.1), (7.2) or Eq.(7.9), depending on the selection of GO electric and magnetic fields. Moreover, one can evaluate the linearized coherent FO spectrum at a point $\left(x_{o}, y_{o}\right)$, i.e. "CFO" option; and then calculate the focal plane fields by integrating the PWS, as described in Eq. (7.7) or (7.12).

Next in Fig. 7.5b, one should define an observation grid, and choose components of the focal plane fields, i.e. " $E_{x, y, z}$ " and " $H_{x, y, z}$ ". Finally, in Fig. $7.5 \mathrm{c}$, one can decide to use both GO electric and magnetic fields or just one of them, with $(u, v)$ or $(\theta, \phi)$ parameterization. 


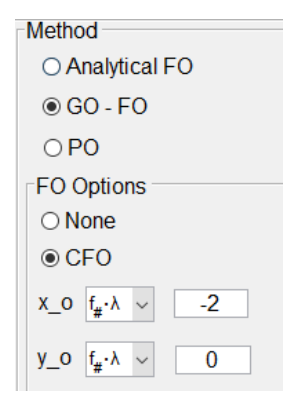

(a)

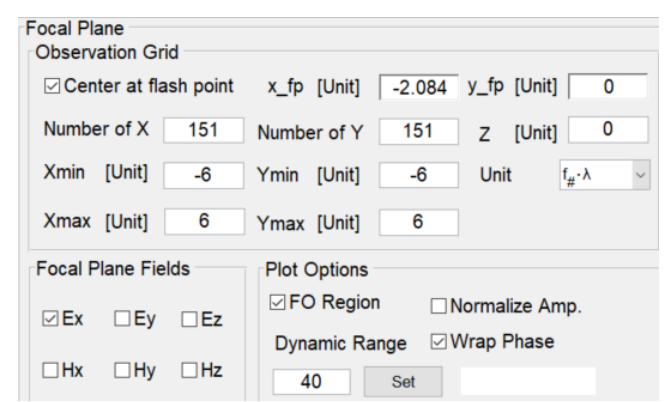

(b)

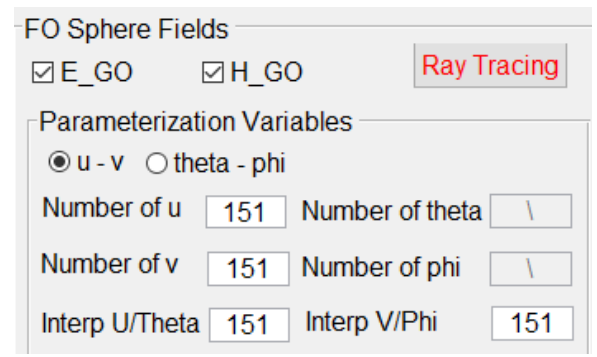

(c)

Figure 7.5: Configurations in the GUI: (a) Calculation methods. (b) Observation grid and components of fields. (c) Selection of GO fields and parameterization variables.

\subsubsection{GO fields and focal plane fields calculated by the GUI}

Here we take an elliptical silicon $\left(\varepsilon_{r}=11.9\right)$ lens as an example. The lens has the diameter of $D_{l}=5 \mathrm{~mm}$ and the f-number of $f_{\#}^{l}=0.6$. A quarter-wavelength matching layer at $480 \mathrm{GHz}$ made of Parylene $\left(\varepsilon_{m}=2.62\right)$ is applied. And the incident field is a unitary TM polarized plane wave operated at $720 \mathrm{GHz}$ with the skew angle of $\theta_{s}=10^{\circ}, \phi_{s}=0^{\circ}$.

In this case, Fig. 7.6 shows the GO ray fields on the FO sphere. GO electric field (Fig. 7.6a) is divided into $E_{\theta}$ and $E_{\phi}$ components, and GO magnetic field (Fig. 7.6b) is divided into $H_{\theta}$ and $H_{\phi}$ components. As it can be seen, both amplitude and phase are shown.

By using the GO electric and magnetic fields, the focal plane fields are evaluated and plotted in Fig. 7.7. Here the incident plane wave is TM polarized. Therefore, we only show the dominant component, $E_{x}$. Moreover, the 3D coherent FO field is plotted in the inset. In Fig. 7.7, we use the PO analysis as the reference, and compare the analytical FO, GO-FO, and coherent FO, for both the amplitude and the phase. It should be mentioned that the GUI can only use one approach at a time. Here we put all approaches together for comparison purpose. 


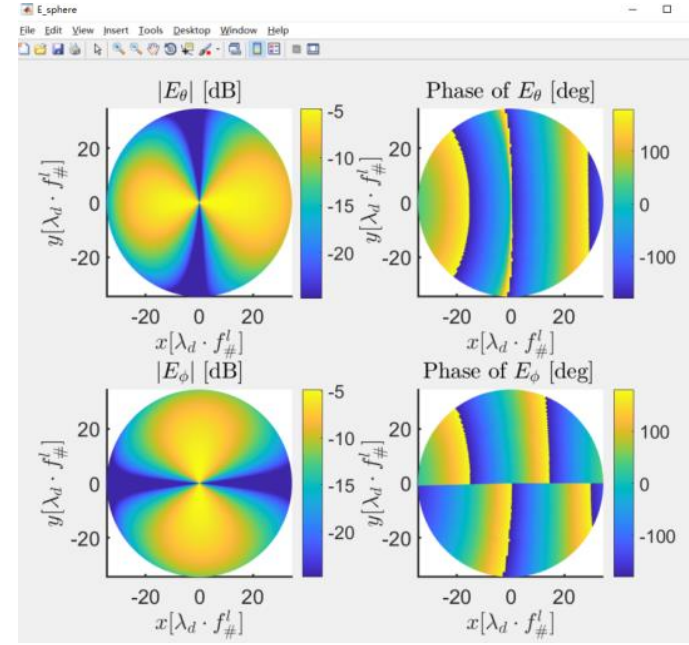

(a)

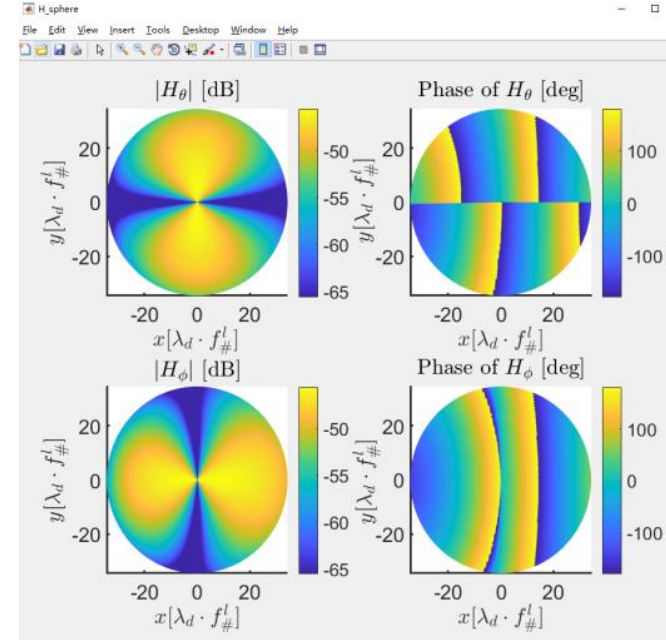

(b)

Figure 7.6: GO ray fields calculated by the GUI: (a) GO electric field. (b) GO magnetic field.
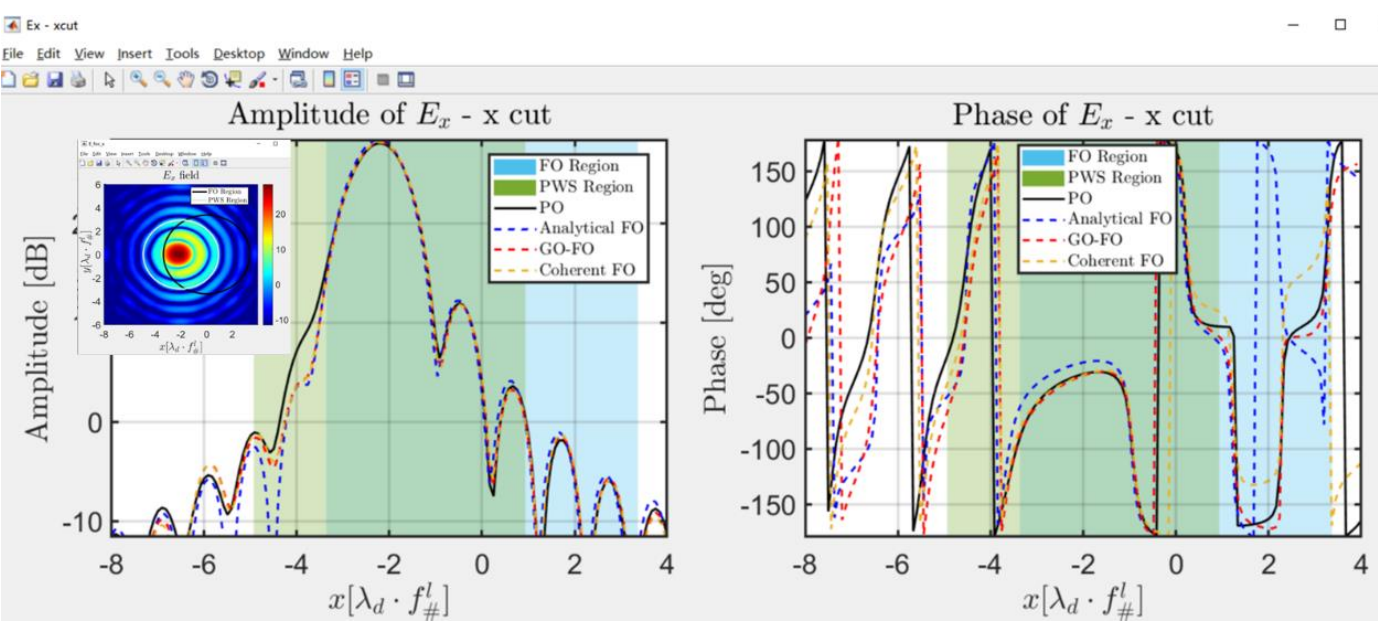

Figure 7.7: Focal plane fields evaluated by the GUI.

\subsection{Antenna-coupled QO systems}

A second GUI is built to analyze antenna-coupled QO systems, as shown in Fig. 7.8. As it can be seen, there are three areas, namely "Incident fields", "Feed antenna", and "Calculation \& Performance". In this section, we will discuss these areas and explain how the GUI calculates the power delivered to the load of an antenna. In subsection 7.5.1, incident fields used in this GUI are discussed. Next in subsection 7.5.2, the procedure to obtain the fields radiated by an antenna is explained. Finally, in subsection 7.5.3, the reaction integral and the calculation of common efficiency terms used in the GUI are listed. 


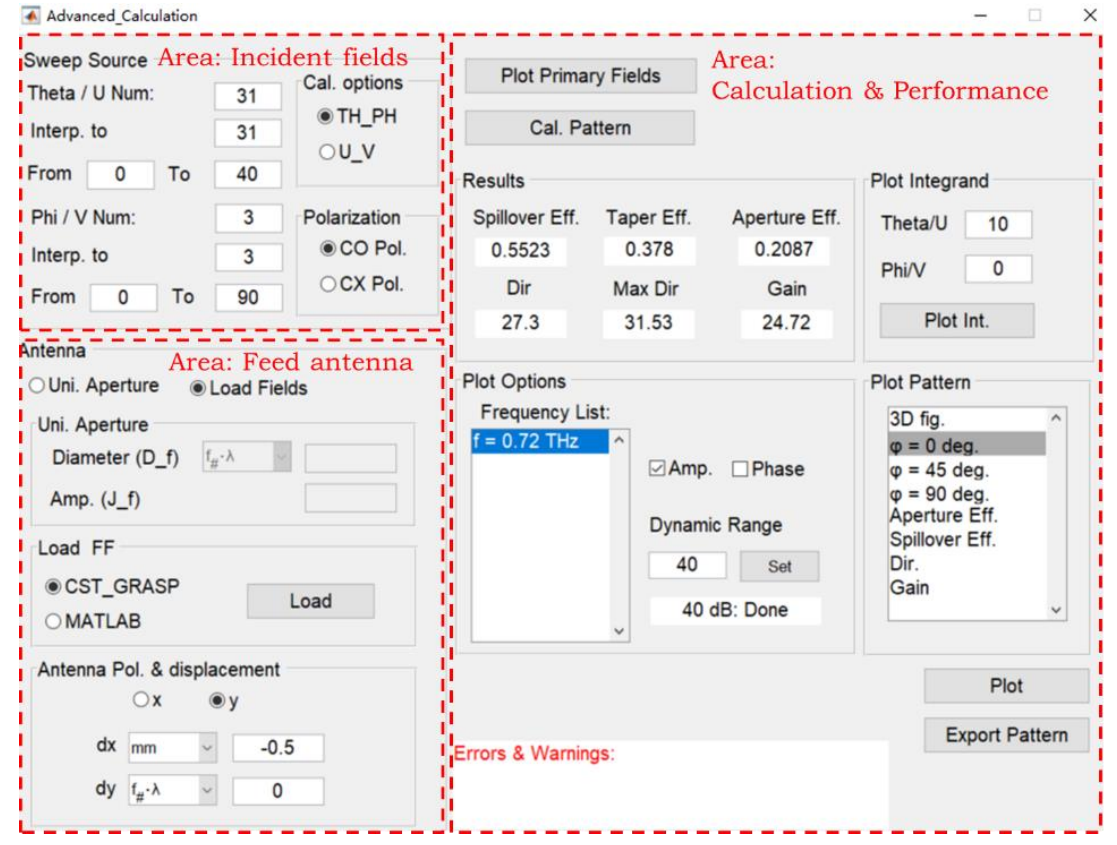

Figure 7.8: Layout of the second GUI for analyzing antenna-coupled QO systems, which is divided into three areas.

\subsubsection{Incident fields}

Two types of incident fields are discussed in section 2.2.1: A plane wave and a Huygens source. For a plane wave incidence, we define the polarization of the plane wave by using the LudwigIII definition, as described in Eq. (2.23) and (2.24). In the second GUI, we relate the reference polarization (Co-Pol.) to the antenna polarization as:

$$
\left\{\begin{array}{l}
\hat{p}_{i, C o}=\cos \phi_{s} \hat{\theta}_{s}-\sin \phi_{s} \hat{\phi}_{s}: x-\text { pol. Antenna } \\
\hat{p}_{i, C o}=\sin \phi_{s} \hat{\theta}_{s}+\cos \phi_{s} \hat{\phi}_{s}: y-\text { pol. Antenna }
\end{array}\right.
$$

For a Huygens source, the electric dipole can be $\mathrm{x}$ - or $\mathrm{y}$ - polarized, which depends on the orientation angle $\gamma$ :

$$
\left\{\begin{array}{l}
\gamma=0^{\circ}: \hat{p}_{i, C o}=\hat{x}: x-\text { pol. Antenna } \\
\gamma=90^{\circ}: \hat{p}_{i, C o}=\hat{y}: y-\text { pol. Antenna }
\end{array}\right.
$$

Moreover, the incident fields incoming from different skew angles can be defined by two sets of variables: $\left(\theta_{s}, \phi_{s}\right)$ and $\left(u_{s}, v_{s}\right)$, which are related to each other as:

$$
\theta_{s}=\operatorname{asin} \sqrt{u_{s}^{2}+v_{s}^{2}}, \phi_{s}=\operatorname{atan} \frac{v_{s}}{u_{s}}
$$

It has been mentioned in section 6.4.1 that $\left(u_{s}, v_{s}\right)$ variables are preferred to display a pattern. One can define a rectangular $u_{s}-v_{s}$ grid and display the pattern conveniently. 


\subsubsection{Fields radiated by an antenna}

In order to calculate the reaction integral for open-circuit voltage, one needs to evaluate two sets of sources: GO fields on a FO sphere and the fields radiated by a feed antenna. The GO fields have been discussed. In this subsection, we mainly focus on evaluating the fields radiated by the antenna.

\section{- Antenna propagation}

The tool is able to load the fields radiated by an antenna from external files like CST-GRASP cuts or MATLAB matrices. In such files, the fields are always assumed to be broadside radiated, meaning the feed antenna is placed at the center of the reference system, as depicted in Fig. 7.9. Here we assume a far-field (FF) radiation and the radiated fields are recorded on an FF sphere $S_{F F}$ with the radius of $R_{F F}$. Therefore, the electric field radiated by the antenna on $S_{F F}$ can be expressed as a spherical wave:

$$
\vec{E}_{a}^{T x}\left(\theta_{F F}, \phi_{F F}\right)=\left(E_{a}^{T x, \perp} \hat{\phi}_{F F}+E_{a}^{T x, \|} \hat{\theta}_{F F}\right) \cdot \frac{e^{-j k R_{F F}}}{R_{F F}}
$$

where $E_{a}^{T x, \perp}$ and $E_{a}^{T x, \|}$ are TE and TM components of the radiated field, respectively. Moreover, the magnetic field is related to the electric field as:

$$
\vec{H}_{a}^{T x}=\frac{1}{\zeta} \hat{s}_{i, F F} \times \vec{E}_{a}^{T x}
$$

To calculate the reaction integral, the radiated fields on a FO sphere are needed. Therefore, one should evaluate the fields captured by the FO sphere, i.e. $\vec{E}_{a}^{T x}\left(\theta_{F O}, \phi_{F O}\right)$, which can be calculated by back/forward propagating $\vec{E}_{a}^{T x}\left(\theta_{F F}, \phi_{F F}\right)$ as:

$$
\vec{E}_{a}^{T x}\left(\theta_{F O}, \phi_{F O}\right)=\vec{E}_{a}^{T x}\left(\theta_{F F}, \phi_{F F}\right) \cdot R_{F F} \cdot e^{j k R_{F F}} \cdot \frac{e^{-j k R_{F O}}}{R_{F O}}
$$

It should be noticed that since $\left(\theta_{F F}, \phi_{F F}\right)$ and $\left(\theta_{F O}, \phi_{F O}\right)$ are two sets of variables, one should use interpolation to numerically transform $\left(\theta_{F F}, \phi_{F F}\right)$ to $\left(\theta_{F O}, \phi_{F O}\right)$. The same step can also be done for the magnetic field. 


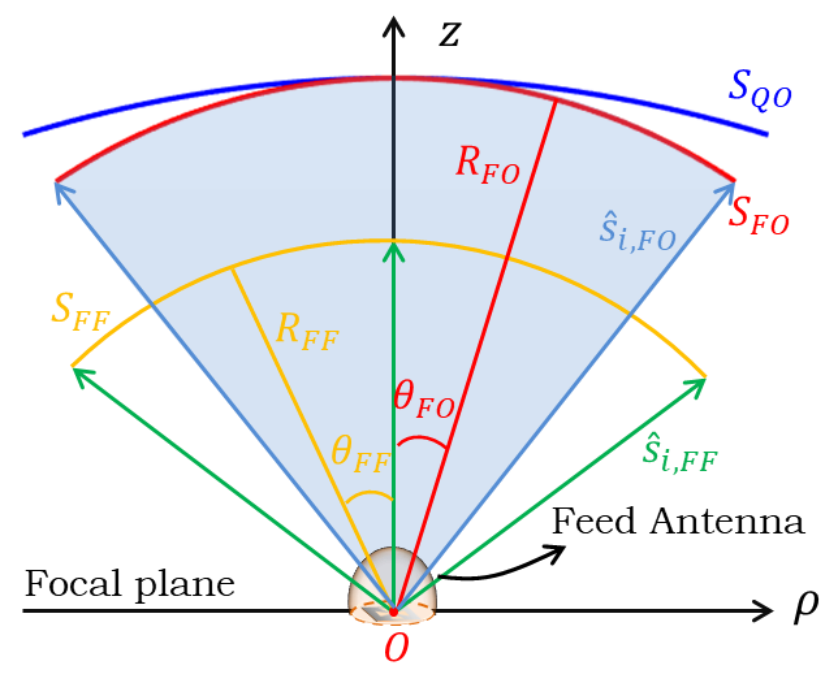

Figure 7.9: A schematic representation of broadside radiation for a central feed.

Next, we consider shifting the antenna within the focal plane of the QO component, with a distance $\vec{d}_{a}=d x \hat{x}+d y \hat{y}$, as depicted in Fig. 7.10. In this case, the reference system is located at the center of the focal plane, $O$. The FO sphere in this system is parameterized by the uniform variables $\left(\theta_{F O}, \phi_{F O}\right)$, and a point on it is denoted by $Q_{F O}(x, y, z)$.

And then we introduce a local system centered at the antenna position, $O^{\prime}$. In this system, the FO sphere is parameterized by the non-uniform variables $\left(\theta_{F O}^{\prime}, \phi_{F O}^{\prime}\right)$. To calculate them, we first use the Cartesian variables $\left(x^{\prime}, y^{\prime}, z^{\prime}\right)$ to represent the point on the FO sphere, i.e. $Q_{F O}^{\prime}\left(x^{\prime}, y^{\prime}, z^{\prime}\right)$ :

$$
\left\{\begin{array}{l}
Q_{F O, x^{\prime}}^{\prime}=Q_{F O, x}-d x \\
Q_{F O, y^{\prime}}^{\prime}=Q_{F O, y}-d y \\
Q_{F O, z^{\prime}}^{\prime}=Q_{F O, z}
\end{array}\right.
$$

The radius of the FO sphere then becomes asymmetric, i.e. $R_{F O}^{\prime}=\left|Q_{F O}^{\prime}\left(x^{\prime}, y^{\prime}, z^{\prime}\right)\right|$. By using $Q_{F O}^{\prime}$ and $R_{F O}^{\prime}$, the non-uniform variables $\left(\theta_{F O}^{\prime}, \phi_{F O}^{\prime}\right)$ can be calculated as:

$$
\left\{\begin{array}{l}
\theta_{F O}^{\prime}=\cos ^{-1} \frac{Q_{F O, z^{\prime}}^{\prime}}{R_{F O}^{\prime}} \\
\phi_{F O}^{\prime}=\tan ^{-1} \frac{Q_{F O, y^{\prime}}^{\prime}}{Q_{F O, x^{\prime}}^{\prime}}
\end{array}\right.
$$

Finally, we discuss the fields radiated by the shifted antenna. As mentioned in the central feed case, we only need the fields illuminating the FO sphere, shown as the blue region in Fig. 7.10. Therefore, by interpolating the broadside field $\vec{E}_{a}^{T x}\left(\theta_{F F}, \phi_{F F}\right)$ into the off-broadside one $\vec{E}_{a}^{T x}\left(\theta_{F O}^{\prime}, \phi_{F O}^{\prime}\right)$ and considering the back/forward propagation: 


$$
\vec{E}_{a}^{T x}\left(\theta_{F O}, \phi_{F O}\right)=\left[\vec{E}_{a}^{T x}\left(\theta_{F O}^{\prime}, \phi_{F O}^{\prime}\right) \cdot R_{F F} \cdot e^{j k R_{F F}}\right] \cdot \frac{e^{-j k R_{F O}^{\prime}}}{R_{F O}^{\prime}}
$$

The same steps can also be done to derive the magnetic field $\vec{H}_{a}^{T x}\left(\theta_{F O}, \phi_{F O}\right)$.

It is worth mentioning that Eq. (7.24) is derived under the assumption that the FO sphere is in the far-field region of the antenna. Consequently, this method is not applicable when the FO sphere is in the near-field region. In such cases, the tool can either directly import the offbroadside near fields on the FO sphere, or still use Eq. (7.24) with a reduction in the accuracy.

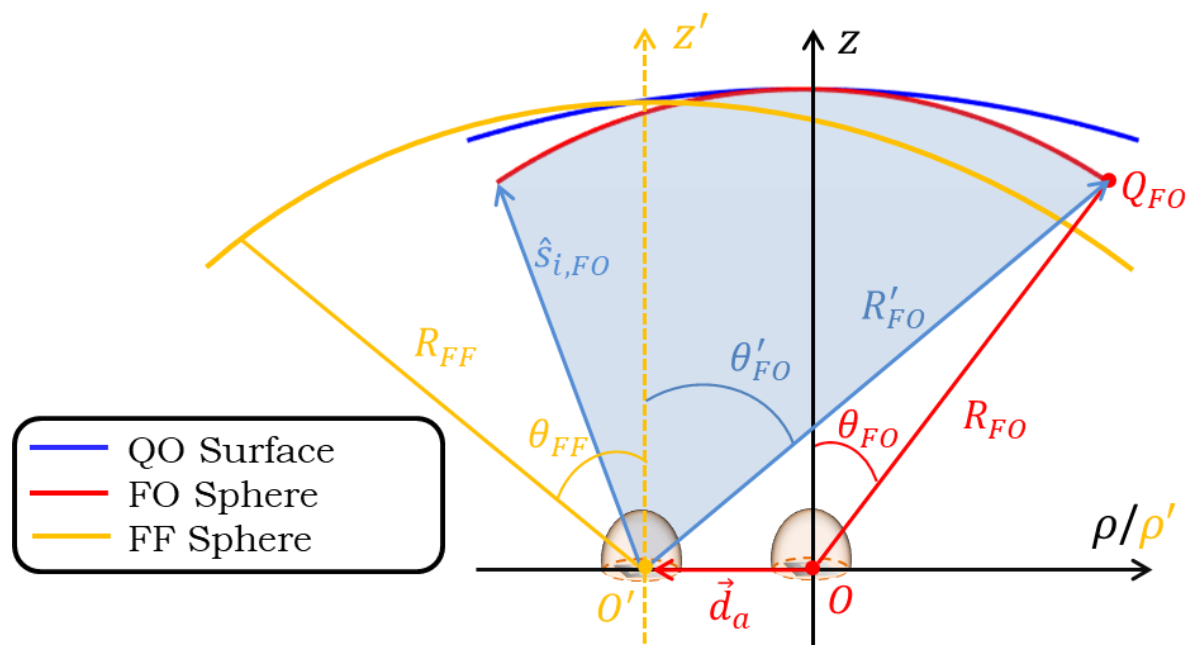

Figure 7.10: A schematic representation of off-broadside radiation for a displaced feed.

\section{- Examples of fields radiated by an antenna}

In the second GUI, there is an area used to load fields radiated by an antenna, as shown in Fig 7.11a. One can define a uniform aperture by inputting the diameter and the amplitude of the aperture. Also, one can import the fields from external files, like CST-GRASP cuts or MATLAB matrices. The polarization of the antenna is " $x$ " or " $y$ ", which decides the reference polarization (Co-Pol.) of the primary field:

$$
\left\{\begin{array}{l}
\hat{e}_{a, c o}^{T x}=\cos \left(\phi_{F F}\right) \hat{\theta}_{F F}-\sin \left(\phi_{F F}\right) \hat{\phi}_{F F}: x-\text { pol. Antenna } \\
\hat{e}_{a, c o}^{T x}=\sin \left(\phi_{F F}\right) \hat{\theta}_{F F}+\cos \left(\phi_{F F}\right) \hat{\phi}_{F F}: y-\text { pol. Antenna }
\end{array}\right.
$$

and the displacement of the antenna is $\vec{d}_{a}=d x \hat{x}+d y \hat{y}$. After loading the fields radiated by the antenna, one can plot them in an external window, Fig 7.11b. In this window, one can plot the EM fields on a far-field sphere, as described in Eq. (7.19); or the fields on a FO sphere, as described in Eq. (7.24). 


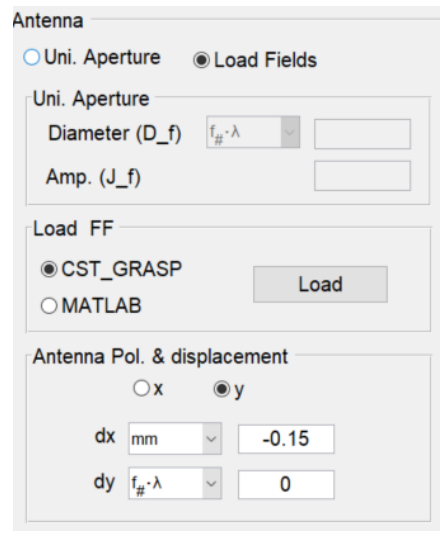

(a)

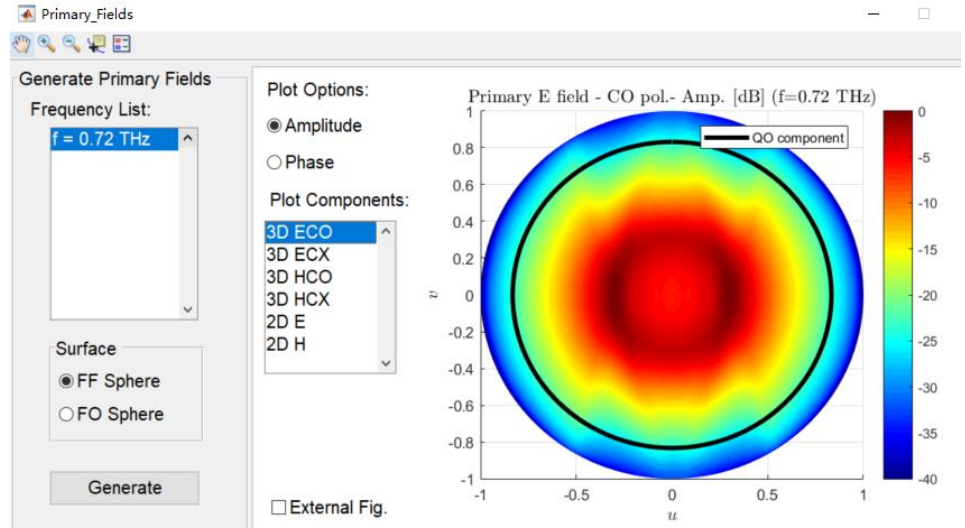

(b)

Figure 7.11: Screenshots of the second GUI: (a) Area used to define an antenna. (b) External window used to plot fields radiated by the defined antenna.

Here we take an elliptical silicon $\left(\varepsilon_{r}=11.9\right)$ lens as an example to show the loaded primary fields inside the lens. The lens has the diameter of $D_{l}=5 \mathrm{~mm}$ and the f-number of $f_{l}^{l}=0.6$. A quarter-wavelength matching layer at $480 \mathrm{GHz}$ made of Parylene $\left(\varepsilon_{m}=2.62\right)$ is applied. The antenna is a y-polarized leaky-wave antenna operated at $720 \mathrm{GHz}$ [51], with a displacement $d x=-0.15 \mathrm{~mm}$. Fig. 7.12 shows the Co-Pol. component of the primary electric fields, evaluated on a FF sphere with the radius of $R_{F F}=1 \mathrm{~m}$ (Fig. 7.12a) and on the FO sphere of the lens (Fig. 7.12b). As it can be seen in Fig. 7.12a, the antenna radiates most of the field within the rim angle subtended by the lens. Moreover, in Fig. 7.12b, the main beam position moves due to the displacement of the antenna.

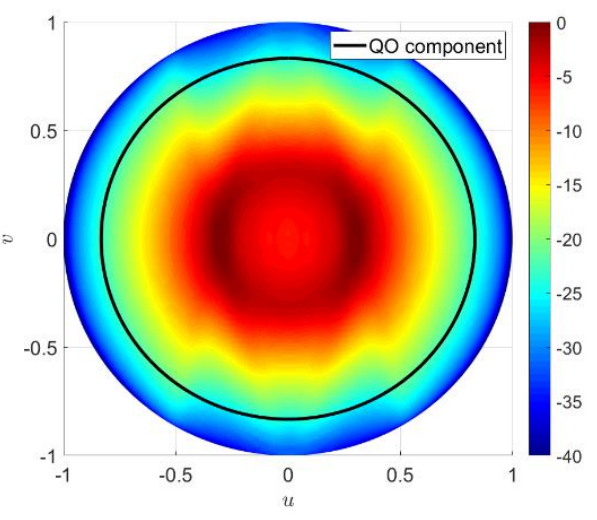

(a)

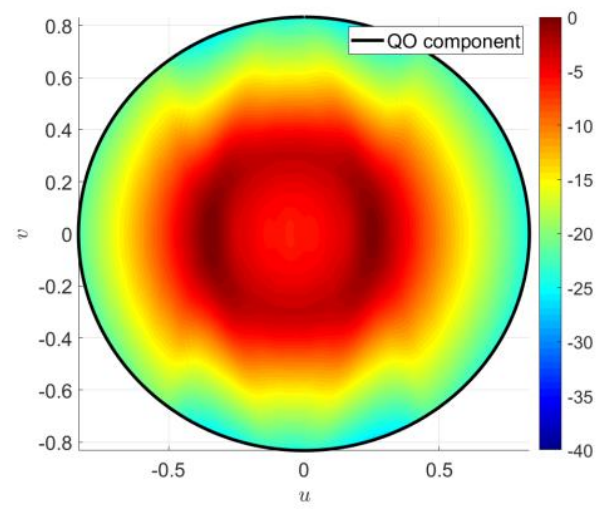

(b)

Figure 7.12: Co-Pol. component of the primary electric fields evaluated on: (a) A FF sphere with the radius of $R_{F F}=1 \mathrm{~m}$. (b) The FO sphere of the lens. The black circle is the boundary of the lens. 


\subsubsection{Power delivered to an antenna load and common efficiency terms}

\section{- Power delivered to an antenna load}

It has been discussed in chapter 6 that for an antenna-coupled QO system, the open-circuit voltage of the antenna can be evaluated by calculating the reaction between the fields in transmission and the induction currents on a FO sphere:

$$
V_{o c}\left(\theta_{S}, \phi_{S}\right)=\iint_{S_{F O}}\left[\vec{H}_{a}^{T x} \cdot \vec{M}_{G O}-\vec{E}_{a}^{T x} \cdot \vec{J}_{G O}\right] d S
$$

where $\vec{J}_{G O}=\hat{n} \times \vec{H}_{G O}, \vec{M}_{G O}=\vec{E}_{G O} \times \hat{n}$, and $\hat{n}$ is the normal vector of the FO sphere. It is worth noting that Eq. (7.26) does not apply any approximation; therefore, it can be used also when the QO system is in the near field of the antenna and for very large skewed angles. In the GUI, Eq. (7.26) is implemented due to its generality. Moreover, the power delivered to the load of the antenna is evaluated as follows:

$$
P_{L}\left(\theta_{s}, \phi_{s}\right)=\frac{\left|\int_{0}^{2 \pi} \int_{0}^{\theta_{0}}\left[\vec{H}_{a}^{T x} \cdot \vec{M}_{G O}-\vec{E}_{a}^{T x} \cdot \vec{J}_{G O}\right] \sin \theta d \theta d \phi\right|^{2}}{16 \int_{0}^{2 \pi} \int_{0}^{\pi}\left|\vec{W}_{\text {rad }} \cdot \hat{n}\right| \sin \theta d \theta d \phi}
$$

where $\theta_{0}$ is the rim angle of the QO surface, and $\vec{W}_{\text {rad }}=\frac{1}{2} \operatorname{Re}\left(\vec{E}_{a}^{T x} \times \vec{H}_{a}^{T x *}\right)$ is the timeaverage radiated power density.

\section{- Common efficiency terms}

In Eq. (6.31), the taper efficiency $\eta_{t}^{R x}$ is calculated by using the directivity $D_{i r}^{R x}$. However, $D_{i r}^{R x}$ can be obtained only when the complete pattern is evaluated, which is time-consuming. Therefore, in the second GUI, we use another way to efficiently calculate $\eta_{t}^{R x}$ and $D_{i r}^{R x}$. In the GUI, the aperture efficiency in reception is calculated as:

$$
\eta_{a p}^{R x}=\frac{P_{L}}{P_{\text {inc }}}
$$

where $P_{L}$ is the power delivered to an antenna load and $P_{\text {inc }}$ is the incident power. For the spillover efficiency in reception, $\eta_{s o}^{R x}$, due to the reciprocity, we can assume it is the same as the one in transmission, i.e $\eta_{s o}^{R x}=\eta_{s o}^{T x}$. Therefore, the taper efficiency in reception can be evaluated efficiently as:

$$
\eta_{t}^{R x}=\eta_{a p}^{R x} / \eta_{s o}^{T x}
$$

Furthermore, the directivity in reception can also be obtained efficiently as:

$$
D_{i r}^{R x}=D i r_{\max } \cdot \eta_{t}^{R x}
$$

where $D_{i r}^{\max }$ is the maximum theoretical directivity. 


\section{- Performance in reception evaluated by the GUI}

Here we use the GUI to evaluate the performance of the leaky lens antenna introduced in subsection 7.5.2. Fig. 7.13 shows the performance of the leaky lens antenna evaluated by the GUI. The common efficiency terms, the directivity, and the gain are shown in Fig. 7.13a, marked by the red dashed box. The Co-Pol. power pattern is plotted in $\left(u_{s}, v_{s}\right)$ with 3D (Fig. 7.13b) and $u_{s}$ cut (Fig. 7.13c). Moreover, there is an option to plot the phase of the open-circuit voltage, i.e. $\angle V_{o c}$, as shown in Fig. 7.13d. In addition, one can plot the integrand of the reaction integral described in Eq. (7.26), i.e. $\vec{H}_{a}^{T x} \cdot \vec{M}_{G O}-\vec{E}_{a}^{T x} \cdot \vec{J}_{G O}$. This can be done for a specific incident skew angle; and in Fig. 7.13e, we choose $\theta_{s}=10^{\circ}, \phi_{s}=0^{\circ}$. 


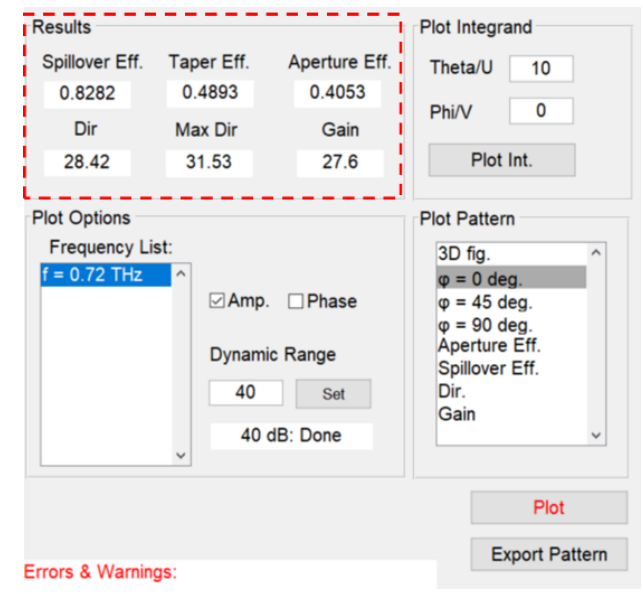

(a)

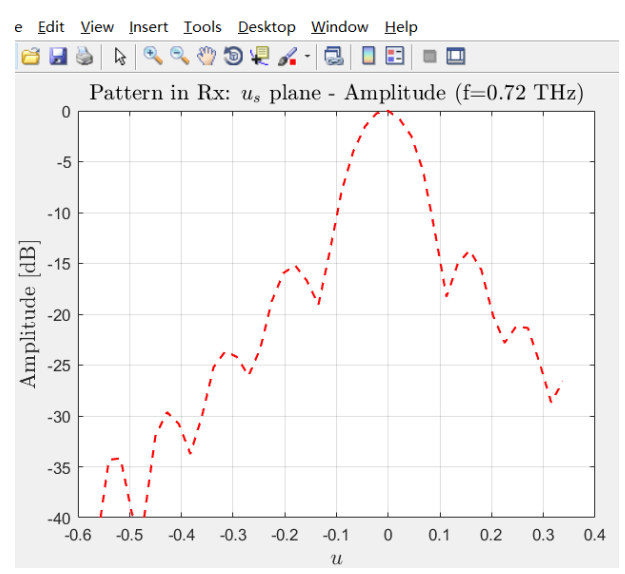

(c)

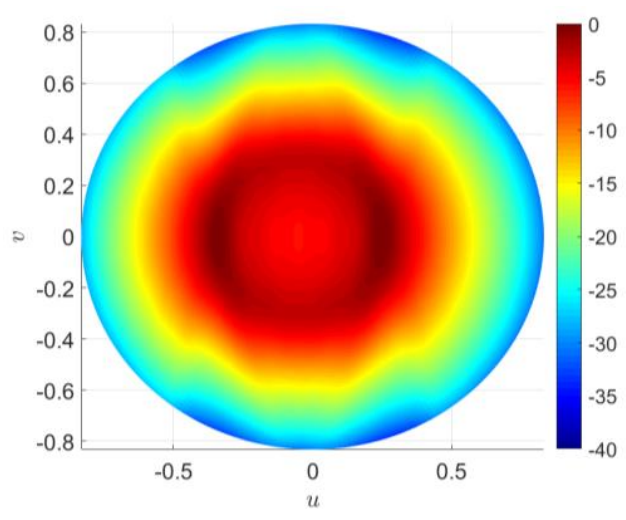

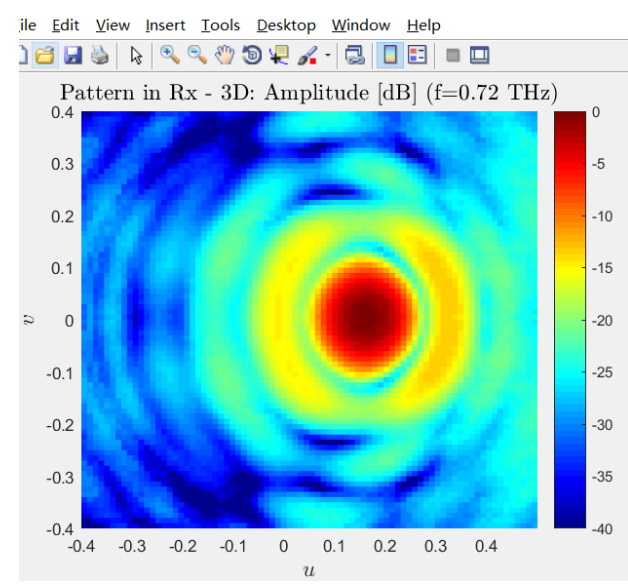

(b)

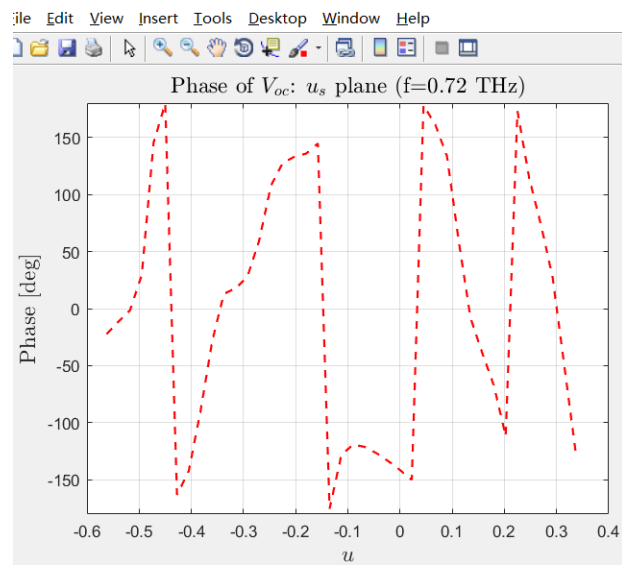

(d)

(e)

Figure 7.13: The performance of the leaky lens antenna evaluated by the GUI: (a) Common efficiency terms, directivity, and gain. (b) 3D power pattern (Co-Pol.). (c) $u_{s}$ cut of the pattern. (d) Phase of $V_{o c}$. (e) Integrand of the reaction integral for the incident skew angle $\theta_{s}=10^{\circ}, \phi_{s}=0^{\circ}$. 
In the GUI, it is also possible to analyze the case of multiple frequencies. For the same leaky lens antenna, we choose three frequency points: $f=0.24,0.48,0.72 \mathrm{THz}$. In this case, one can select a specific frequency in the frequency list to plot the pattern, as shown in Fig. 7.14a. Moreover, the efficiency terms, the directivity, and the gain are functions of frequency, as shown in Fig. 7.14b for the aperture efficiency.

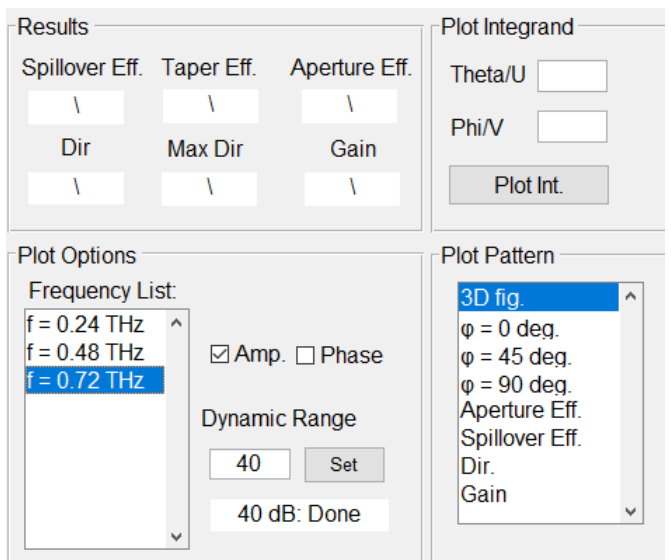

(a)

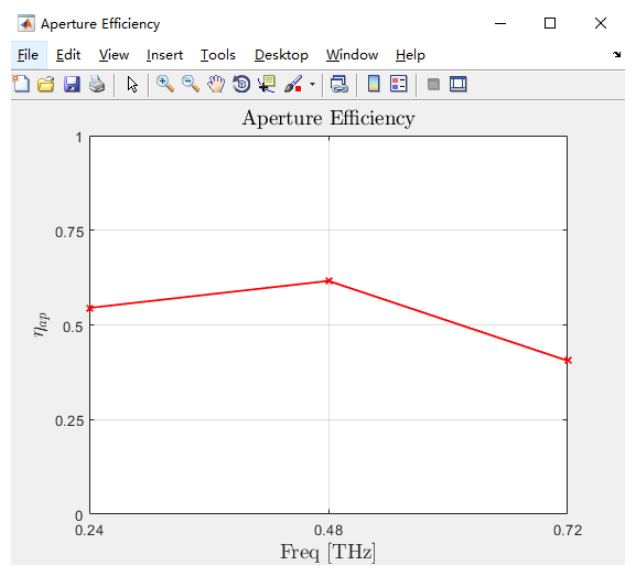

(b)

Figure 7.14: The leaky lens antenna with three frequency points, $f=0.24,0.48,0.72 \mathrm{THz}$ : (a) Frequency list. (b) Aperture efficiency.

\subsection{Conclusion}

To conclude, this GUI tool represents a GO/FO based tool that can be used to analyze and design QO systems in reception. It has the following outputs:

(1) Five commonly used QO components are included to improve design possibilities. Their 2D geometries can be plotted and exported.

(2) To preliminarily analyze QO systems, a ray tracing code is developed to visualize ray propagation. For all QO components, ray tracing figures can be plotted.

(3) The coherent FO method is implemented and validated. The tool can evaluate and export coherent FO spectra. Moreover, the focal plane fields calculated by using these spectra can be plotted and exported.

(4) The tool is able to load and plot fields radiated by an antenna. Moreover, by calculating the reaction integral, the tool outputs key parameters used to evaluate the performance of antenna-coupled QO systems in reception, including pattern, common efficiency terms, directivity and gain. 


\section{Chapter 8: Conclusion}

\subsection{Summary and conclusion}

In this thesis we discussed a GUI tool for analyzing antenna-coupled QO systems in reception using the FO methodology. This work fulfills the need for a universal tool which implements the FO method for variety of QO components. The current available FO based codes are limited in applicability. The existing codes mainly focus on analyzing parabolic reflectors and elliptical lenses, which leads to small design possibilities. Moreover, their interfaces were not developed with the goal to be user-friendly for designing purposes. The proposed tool solves these constrains. The tool provides five widely used QO components to improve design possibilities. Also, the graphical user interface is informative. Users can easily operate the tool without extensive knowledge about the FO methodology. In addition, the possibility for designing matching layers is included for dielectric lenses, which is essential in practical dielectric lens designs.

The GUI tool is mainly based on evaluating the GO fields on a FO sphere. Besides evaluating the GO ray fields, analytical GO fields are also derived to increase the speed of the tool. Once GO fields are obtained, coherent FO spectra can also be evaluated. Moreover, for an antenna-coupled QO system in reception, the reaction between the GO fields and the fields radiated by the antenna leads to the power delivered to the antenna. This FO analysis in reception is convenient when analyzing multi-mode antenna configurations. More importantly, it provides insight in synthesizing antennas. In addition, the tool is tested and validated by using CST and GRASP full-wave simulation software. Therefore, it can be concluded that this tool represents a GO/FO based tool that can be used for analyzing and designing antenna-coupled QO systems in reception.

In chapter 2 we discussed the most widely used QO components in reception scenarios. Five canonical QO components were included. We explained the applications of each one in $\mathrm{THz}$ systems. These components are then parameterized by defining truncation angle, fnumber, radial distance, and normal vector. Moreover, we discussed the ray tracing technique that can be used to describe reception scenarios for the QO components. In the ray tracing, incident rays were defined; and by using the laws of reflection and refraction, the reflected and transmitted rays were evaluated. In addition, in order to reduce the reflection coefficient for dielectric lenses, the case of adding a matching layer was investigated. 
In the third chapter, we applied the FO method to derive the PWS of the direct field focalized by a QO component. To start with, the PO analysis was introduced. It was implemented as validation on focal plane fields. More importantly, by using the FO approximations, the PO radiation integral was approximated by the FO integral where the focal plane field was evaluated by integrating the fields on a FO sphere, referred to as the GO fields. Moreover, the applicability region for those approximations was discussed. To maximize this region, the radius of the FO sphere was chosen as large as possible for each QO component.

When the FO integral was written as a spectral representation, we found the focal plane field could be represented by a spectral function with a quadratic phase term. It was explained that this phase term cannot be neglected when the observation was far away from the focus. In such a case, we discussed a coherent FO method to include this phase term in the spectrum of the focal plane field. We showed that by applying FT on this quadratic phase term, the full coherent FO spectrum could be evaluated by calculating a convolution integral. Furthermore, we introduced a linearization approximation on the quadratic phase term to simplify this convolution operation. As a result, the convolution was approximated by a linear shift in the spectral domain, and the full spectrum became a linearized local spectrum.

Chapter 4 focuses on evaluating the fields scattered by a QO component and propagating these fields to the corresponding FO sphere by resorting to the GO technique. Under GO approximation, EM waves can be approximated as tubes of rays propagating in a homogenous medium from one point to another. Moreover, the scattered ray fields follow the laws of reflection and refraction at a two-media separation surface. In order to derive the GO ray expression, we discussed an asymptotic evaluation on the PO radiation integral. By applying the Method of Stationary Phase for reflection and transmission problems, the GO reflected and transmitted fields were derived, respectively.

Subsequently, we showed that it was possible to find analytical expressions for GO ray fields with broadside incidence on QO components. Analytical GO fields were investigated for all QO components, by deriving spreading factor, phase variation, and polarization information. At the end of this section, we checked that analytical GO fields were in excellent agreement with the GO ray fields. Furthermore, we explored the case of slightly off-broadside incidence. In this case, analytical GO fields could be approximated by the ones for broadside illumination multiplied by the linear and coma phase terms, for plane wave incidence; while linear, coma, and compensation phase terms, for point source incidence. Moreover, the limits of the coma phase, where the coma phase term could be neglected, were found and compared for all QO components. 
In chapter 5 numerical examples and validation of GO fields and PWS were shown. The validation was done indirectly by validating the focal plane fields. First the focal plane fields evaluated by using the GO ray fields and the analytical GO fields were compared with the ones obtained using the PO analysis. It was shown that the agreement was very good. Furthermore, numerical examples of PWS were shown to better illustrate the coherent FO method. The full CFO spectrum was compared with the linearized CFO spectrum. Both spectra were accurate but we chose to use the linearized CFO spectrum since it was numerically efficient. Finally, the focal plane fields calculated by using the linearized CFO spectra were validated for all QO components.

In chapter 6 antenna-coupled QO systems were analyzed in reception scenarios. To do so, first the Thevenin equivalent circuit was introduced to represent a system in reception. The open-circuit voltage generator in this circuit was evaluated by calculating the reaction between the fields in transmission and the induction currents on a FO sphere. In the subsequent section, we explained the calculation of the power delivered to an antenna load. The next section discussed the parameters used to evaluate the performance of the systems in reception, including pattern, directivity, gain, and common efficiency terms. Finally, we validated the performance of antenna-coupled QO components in reception. The performance in transmission obtained from CST and GRASP was used as the reference, and the results corresponding to all QO components were validated.

In chapter 7 we described the interface of the developed tool and explained the implementations in it. The GUI tool was divided into two sub-GUIs. In the main GUI, first we described the procedures to define the main QO components and to start a ray tracing analysis. In the next section, we explained the implementation of the coherent FO method in the GUI. The GUI provided two options to evaluate the coherent FO spectra, namely using both GO electric and magnetic fields or using only one of them. Next, the calculation of GO fields used in the GUI was discussed. In addition, numerical examples of GO fields and focal plane fields calculated by the GUI were shown.

Subsequently, the second GUI was introduced. This GUI was used to analyze the performance of antenna-coupled QO systems in reception. It could load the fields radiated by an antenna from external files. In order to evaluate the radiated fields intercepted by a FO sphere, antenna propagation approach was discussed. Moreover, the reaction integral and the calculation of common efficiency terms used in the GUI were shown. Finally, we concluded the outputs of the entire GUI tool built in this thesis. 


\subsection{Future work}

The developed GUI tool is validated and proved to work accurately in analyzing antennacoupled QO systems in reception. However, when analyzing lenses, the execution speed of the tool is relatively low. This is due to the fact that the interpolation procedure involved in calculating the GO ray fields is relatively slow. To solve this issue, a fast interpolation routine could be developed in MATLAB specially for this problem; or a parallel computation code could be implemented. Moreover, we have shown that the accuracy of the GO ray fields for hyperbolic lenses decreases for small f-number cases. Therefore, current calculation could be improved. In addition, when deriving the analytical GO fields for hyperhemispherical lenses, we only consider the broadside incidence. In the future, slightly off-broadside case could also be investigated. Furthermore, the GUI could be extended to evaluate the performance of absorber-coupled QO systems.

For the GUI itself, the robustness of the code could be improved by following more extensive tests. There could be bugs needed to be fixed. Also, the GUI could be improved according to the suggestions from users. Finally, the GUI could be packed as a software, which is more convenient to users. 


\section{Bibliography}

[1] R. Appleby and H. B. Wallace, "Standoff detection of weapons and contraband in the 100 GHz to $1 \mathrm{THz}$ region," IEEE transactions on antennas and propagation, vol. 55, no. 11, pp. 2944-2956, 2007.

[2] K. B. Cooper, R. J. Dengler, N. Llombart, B. Thomas, G. Chattopadhyay, and P. H. Siegel, "THz imaging radar for standoff personnel screening," IEEE Transactions on Terahertz Science and Technology, vol. 1, no. 1, pp. 169-182, 2011.

[3] R. Piesiewicz, T. Kleine-Ostmann, N. Krumbholz, D. Mittleman, M. Koch, J. Schoebei, and T. Kurner, "Short-range ultra-broadband terahertz communications: Concepts and perspectives," IEEE Antennas and Propagation Magazine, vol. 49, no. 6, pp. 24-39, 2007.

[4] T. Kleine-Ostmann and T. Nagatsuma, "A review on terahertz communications research," Journal of Infrared, Millimeter, and Terahertz Waves, vol. 32, no. 2, pp. 143-171, 2011.

[5] A. Endo, J. Baselmans, P. van der Werf, B. Knoors, S. Javadzadeh, S. Yates, D. Thoen et al., "Development of DESHIMA: a redshift machine based on a superconducting on-chip filterbank," in Millimeter, Submillimeter, and Far-Infrared Detectors and Instrumentation for Astronomy VI, 2012, vol. 8452, p. 84520X: International Society for Optics and Photonics.

[6] J. Baselmans, J. Bueno, S. J. Yates, O. Yurduseven, N. Llombart, K. Karatsu, A. Baryshev et al., "A kilo-pixel imaging system for future space based far-infrared observatories using microwave kinetic inductance detectors," Astronomy \& Astrophysics, vol. 601, p. A89, 2017.

[7] N. Llombart, S. O. Dabironezare, G. Carluccio, A. Freni, and A. Neto, "Reception Power Pattern of Distributed Absorbers in Focal Plane Arrays: a Fourier Optics Analysis," IEEE Transactions on Antennas and Propagation, 2018.

[8] D. Rutledge and S. Schwarz, "Planar multimode detector arrays for infrared and millimeter-wave applications," IEEE Journal of Quantum Electronics, vol. 17, no. 3, pp. 407-414, 1981.

[9] M. Born and E. Wolf, Principles of optics: electromagnetic theory of propagation, interference and diffraction of light. Elsevier, 2013.

[10] A. S. Glassner, An introduction to ray tracing. Elsevier, 1989.

[11] Z. Yun and M. F. Iskander, "Ray tracing for radio propagation modeling: Principles and applications," IEEE Access, vol. 3, pp. 1089-1100, 2015.

[12] Zemax. Opticstudio - zemax [Online]. Available: http://www.zemax.com/os/opticstudio

[13] C. A. Balanis, Advanced engineering electromagnetics. John Wiley \& Sons, 1999.

[14] J. Gutiérrez-Meana, J. Á. Martínez-Lorenzo, and F. Las-Heras, "High frequency techniques: The physical optics approximation and the modified equivalent current approximation (MECA)," in Electromagnetic waves propagation in complex matter: InTech, 2011.

[15] N. Llombart, B. Blázquez, A. Freni, and A. Neto, "Fourier optics for the analysis of distributed absorbers under thz focusing systems," IEEE Transactions on Terahertz Science and Technology, vol. 5, no. 4, pp. 573-583, 2015.

[16] S. O. Dabironezare, G. Carluccio, A. Neto, and N. Llombart, "Coherent Fourier Optics Analysis of Large Format-Lens Based Focal Plane Arrays," in 12th European Conference on Antennas and Propagation, London, United Kingdom, April, 2018.

[17] O. Yurduseven, A. Freni, N. Llombart, and A. Neto, "On the Use of Thevenin Circuits in Distributed Transmission Lines and Its Consequences for Antennas in Reception," in The 10th European Conference on Antennas and Propagation (EuCAP), Davos, Switzerland, April, 2016. 
[18] R. Bustos, M. Rubio, A. Otárola, and N. Nagar, "Parque Astronómico de Atacama: An ideal site for millimeter, submillimeter, and mid-infrared astronomy," Publications of the Astronomical Society of the Pacific, vol. 126, no. 946, p. 1126, 2014.

[19] J. Lebreton and D. Matson, "Cassini/Huygens Mission," Encyclopedia of Astronomy and Astrophysics, 2002.

[20] P. Hannan, "Microwave antennas derived from the Cassegrain telescope," IRE Transactions on Antennas and Propagation, vol. 9, no. 2, pp. 140-153, 1961.

[21] G. M. Besenyei, "Reflector antenna designs for airborne radar applications," AIR FORCE INST OF TECH WRIGHT-PATTERSON AFB OH1988.

[22] D. B. Rutledge, D. P. Neikirk, and D. P. Kasilingam, "Integrated-circuit antennas," Infrared and millimeter waves, vol. 10, no. part 2, pp. 1-90, 1983.

[23] G. M. Rebeiz, "Millimeter-wave and terahertz integrated circuit antennas," Proceedings of the IEEE, vol. 80, no. 11, pp. 1748-1770, 1992.

[24] D. F. Filipovic, S. S. Gearhart, and G. M. Rebeiz, "Double-slot antennas on extended hemispherical and elliptical silicon dielectric lenses," IEEE Transactions on microwave theory and techniques, vol. 41, no. 10, pp. 1738-1749, 1993.

[25] L. Ferrari, O. Yurduseven, N. Llombart, S. J. Yates, J. Bueno, V. Murugesan, D. J. Thoen et al., "Antenna Coupled MKID Performance Verification at $850 \mathrm{GHz}$ for Large Format Astrophysics Arrays," IEEE Transactions on Terahertz Science and Technology, vol. 8, no. 1, pp. 127-139, 2018.

[26] T. Han and Z. Wu, "Planar hyperlens with homogeneous parameters based on linear optical transformation," EPL (Europhysics Letters), vol. 107, no. 3, p. 34002, 2014.

[27] C. Lv, W. Li, X. Jiang, and J. Cao, "Far-field super-resolution imaging with a planar hyperbolic metamaterial lens," EPL (Europhysics Letters), vol. 105, no. 2, p. 28003, 2014.

[28] B. Gompf, M. Gerull, T. Müller, and M. Dressel, "THz-micro-spectroscopy with backward-wave oscillators," Infrared physics \& technology, vol. 49, no. 1-2, pp. 128-132, 2006.

[29] H. Rehn, "Optical properties of elliptical reflectors," Optical Engineering, vol. 43, no. 7, pp. 1480-1489, 2004.

[30] M. R. Benson and M. A. Marciniak, "Design considerations regarding ellipsoidal mirror based reflectometers," Optics express, vol. 21, no. 23, pp. 27519-27536, 2013.

[31] H.-T. Chou, L.-R. Kuo, H.-H. Chou, K.-L. Hung, and P. Nepa, "Realistic implementation of ellipsoidal reflector antennas to produce near-field focused patterns," Radio Science, vol. 46, no. 05, pp. 1-10, 2011.

[32] S. C. Hagness, A. Taflove, and J. E. Bridges, "Two-dimensional FDTD analysis of a pulsed microwave confocal system for breast cancer detection: Fixed-focus and antennaarray sensors," IEEE transactions on biomedical engineering, vol. 45, no. 12, pp. 14701479, 1998.

[33] K. B. Cooper, R. J. Dengler, N. Llombart, A. Talukder, A. V. Panangadan, C. S. Peay, I. Mehdi et al., "Fast high-resolution terahertz radar imaging at 25 meters," in Terahertz Physics, Devices, and Systems IV: Advanced Applications in Industry and Defense, 2010, vol. 7671, p. 76710Y: International Society for Optics and Photonics.

[34] E. Gandini, N. Llombart, and N. Andrea, "Focal Plane Array Size Reduction for Terahertz Transceivers in Integrated Technology," in European conference on Antennas and Propagation (EuCAP), Lisbon, Portugal, 2015.

[35] A. Ludwig, "The definition of cross polarization," IEEE Transactions on Antennas and Propagation, vol. 21, no. 1, pp. 116-119, 1973.

[36] G. Franceschetti, Electromagnetics: theory, techniques, and engineering paradigms. Springer Science \& Business Media, 2013.

[37] B. Blázquez, N. Llombart, D. Cavallo, A. Freni, and A. Neto, "A rigorous equivalent 
network for linearly polarized THz absorbers," IEEE Trans. Antennas Propag, vol. 62, no. 10, pp. 5077-5088, 2014.

[38] V. Rumsey, "On the design and performance of feeds for correcting spherical aberration," IEEE Transactions on Antennas and Propagation, vol. 18, no. 3, pp. 343-351, 1970.

[39] CST. Cst microwave studio - $3 \mathrm{~d}$ em simulation software [Online]. Available: http://www.cst.com/

[40] TICRA. Grasp | ticra [Online]. Available: https://www.ticra.com/products/software/grasp

[41] E. Wolf, "Electromagnetic diffraction in optical systems-I. An integral representation of the image field," Proc. R. Soc. Lond. A, vol. 253, no. 1274, pp. 349-357, 1959.

[42] P. Pathak, "High frequency techniques for antenna analysis," Proceedings of the IEEE, vol. 80, no. 1, pp. 44-65, 1992.

[43] R. Kouyoumjian and P. Pathak, "A Unifm Geometrical Theory of Diffraction for an Edge in a Perfectly Conducting Surface. 62 (11): 1448-1461, 1974," ed: ISSN.

[44] M. Albani, G. Carluccio, and P. H. Pathak, "Uniform ray description for the PO scattering by vertices in curved surface with curvilinear edges and relatively general boundary conditions," IEEE Transactions on Antennas and Propagation, vol. 59, no. 5, pp. 15871596, 2011.

[45] G. Carluccio, M. Albani, and P. H. Pathak, "Uniform asymptotic evaluation of surface integrals with polygonal integration domains in terms of UTD transition functions," IEEE Transactions on Antennas and Propagation, vol. 58, no. 4, pp. 1155-1163, 2010.

[46] A. Constantine Balanies, "Antenna theory analysis and design," ed: John Wiley \& Sons Inc., New York, 1982.

[47] A. Neto, O. Yurduseven, N. Llombart, and A. Freni, "Generalization of the Thevenin Circuit to Antennas Excited by Coherent Fields: Application to Focusing Systems."

[48] S. Schelkunoff, "Some equivalence theorems of electromagnetics and their application to radiation problems," Bell System Technical Journal, vol. 15, no. 1, pp. 92-112, 1936.

[49] R. F. Harrington, Time-harmonic electromagnetic fields. McGraw-Hill, 1961.

[50] O. Yurduseven, N. Llombart, A. Neto, and J. Baselmans, "A dual polarized antenna for THz space applications: antenna design and lens optimization," in Antennas and Propagation Society International Symposium (APSURSI), 2014 IEEE, 2014, pp. 191192: IEEE.

[51] S. Bosma, "Quasi-optical system for the DESHIMA spectrometer," Delft University of Technology, 2017.

[52] A. Neto and N. Llombart, "Wideband localization of the dominant leaky wave poles in dielectric covered antennas," IEEE Antennas and Wireless Propagation Letters, vol. 5, no. $1,2006$. 


\section{Appendix A Parameterizing a QO component}

In this appendix, we will explain more explicitly how we parameterize each QO component. We start with explaining the importance of introducing the $(u, v)$ variable. Furthermore, a more extensive derivation of normal vectors is discussed. Finally, we calculate truncation angle for each QO component.

\section{A.1 Comparison between $(\theta, \phi)$ and $(u, v)$ variables}

Fig. A.1 shows the top view of a parabolic surface sampled by both $(\theta, \phi)$ and $(u, v)$ variables, with $15 \times 15$ sampling points. It can be seen that the $(\theta, \phi)$ variable samples the surface denser around the center and less at the edge; while the $(u, v)$ variable samples the surface uniformly, which means for the same number of sampling points, the $(u, v)$ variable can lead to better convergence than the $(\theta, \phi)$ one.

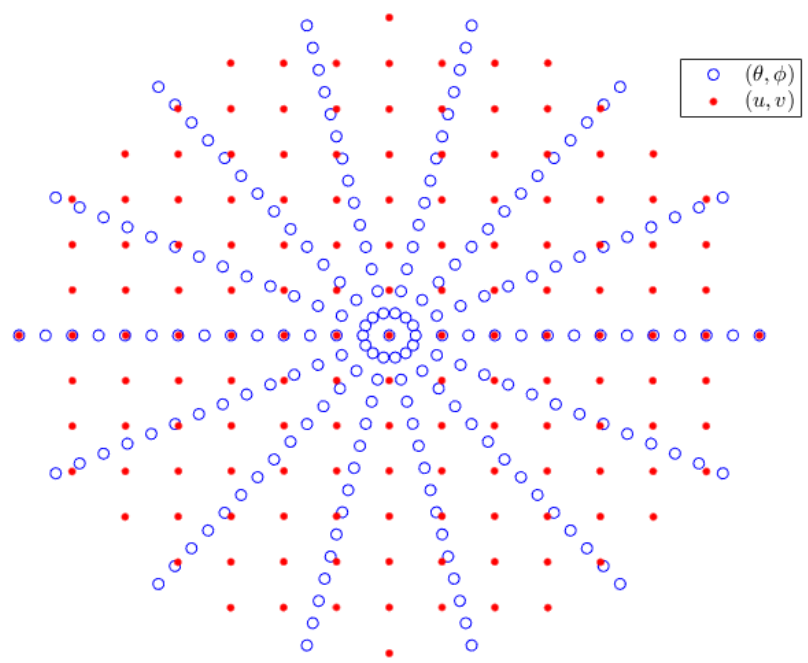

Figure A.1: Top view of a parabolic surface sampled by both $(\theta, \phi)$ and $(u, v)$ variables with $15 \times 15$ points. 


\section{A.2 Derivation of normal vectors for a generic surface}

In Eq. (2.6), we calculate the normal vector of a surface by using a generic expression. In this section, we will expand Eq. (2.6) by calculating the partial derivatives: $\frac{\partial \vec{Q}}{\partial \theta / u}$ and $\frac{\partial \vec{Q}}{\partial \phi / v}$.

- $(\theta, \phi)$ variable

For the $(\theta, \phi)$ variable, the partial derivatives are calculated as follows:

$$
\left\{\begin{array}{l}
\frac{\partial \vec{Q}}{\partial \theta}=\frac{\partial Q_{x}}{\partial \theta} \hat{x}+\frac{\partial Q_{y}}{\partial \theta} \hat{y}+\frac{\partial Q_{z}}{\partial \theta} \hat{z} \\
\frac{\partial \vec{Q}}{\partial \phi}=\frac{\partial Q_{x}}{\partial \phi} \hat{x}+\frac{\partial Q_{y}}{\partial \phi} \hat{y}+\frac{\partial Q_{z}}{\partial \phi} \hat{z}
\end{array}\right.
$$

Each $\theta$-related derivative can be expressed as:

$$
\left\{\begin{array}{l}
\frac{\partial Q_{x}}{\partial \theta}=\frac{\partial r(\theta)}{\partial \theta} \sin \theta \cos \phi+r(\theta) \cos \theta \cos \phi \\
\frac{\partial Q_{y}}{\partial \theta}=\frac{\partial r(\theta)}{\partial \theta} \sin \theta \sin \phi+r(\theta) \cos \theta \sin \phi \\
\frac{\partial Q_{z}}{\partial \theta}=\frac{\partial r(\theta)}{\partial \theta} \cos \theta-r(\theta) \sin \theta
\end{array}\right.
$$

where $\frac{\partial r(\theta)}{\partial \theta}$ depends on the radial distance of a specific surface. While each $\phi$-related derivative can be calculated as:

$$
\left\{\begin{array}{l}
\frac{\partial Q_{x}}{\partial \phi}=-r(\theta) \sin \theta \sin \phi \\
\frac{\partial Q_{y}}{\partial \phi}=r(\theta) \sin \theta \cos \phi \\
\frac{\partial Q_{z}}{\partial \phi}=0
\end{array}\right.
$$

Furthermore, if we define $\left|\vec{n}_{Q}(\theta, \phi)\right|=\left|\frac{\partial \vec{Q}}{\partial \theta} \times \frac{\partial \vec{Q}}{\partial \phi}\right|$, then for any scalar field $f(\theta, \phi)$, a surface integral becomes:

$$
\iint_{S} f(\theta, \phi) d S=\int_{0}^{2 \pi} \int_{0}^{\theta_{0}} f(\theta, \phi)\left|\frac{\partial \vec{Q}}{\partial \theta} \times \frac{\partial \vec{Q}}{\partial \phi}\right| d \theta d \phi
$$

where $\theta_{0}$ is the truncation angle. 
- $(u, v)$ variable

For the $(u, v)$ variable, the partial derivatives are expressed as follows:

$$
\left\{\begin{array}{l}
\frac{\partial \vec{Q}}{\partial u}=\frac{\partial Q_{x}}{\partial u} \hat{x}+\frac{\partial Q_{y}}{\partial u} \hat{y}+\frac{\partial Q_{z}}{\partial u} \hat{z} \\
\frac{\partial \vec{Q}}{\partial v}=\frac{\partial Q_{x}}{\partial v} \hat{x}+\frac{\partial Q_{y}}{\partial v} \hat{y}+\frac{\partial Q_{z}}{\partial v} \hat{z}
\end{array}\right.
$$

Each $u$-related derivative can be calculated as:

$$
\left\{\begin{array}{l}
\frac{\partial Q_{x}}{\partial u}=\frac{\partial r(u, v)}{\partial u} u+r(u, v) \\
\frac{\partial Q_{y}}{\partial u}=\frac{\partial r(u, v)}{\partial u} v \\
\frac{\partial Q_{z}}{\partial u}=\frac{\partial r(u, v)}{\partial u} \sqrt{1-\left(u^{2}+v^{2}\right)}-\frac{r(u, v) u}{\sqrt{1-\left(u^{2}+v^{2}\right)}}
\end{array}\right.
$$

While each $v$-related derivative can be obtained as:

$$
\left\{\begin{array}{l}
\frac{\partial Q_{x}}{\partial v}=\frac{\partial r(u, v)}{\partial v} u \\
\frac{\partial Q_{y}}{\partial v}=\frac{\partial r(u, v)}{\partial v} v+r(u, v) \\
\frac{\partial Q_{z}}{\partial v}=\frac{\partial r(u, v)}{\partial v} \sqrt{1-\left(u^{2}+v^{2}\right)}-\frac{r(u, v) v}{\sqrt{1-\left(u^{2}+v^{2}\right)}}
\end{array}\right.
$$

If we define $\left|\vec{n}_{Q}(u, v)\right|=\left|\frac{\partial \vec{Q}}{\partial u} \times \frac{\partial \vec{Q}}{\partial v}\right|$, then for any scalar field $f(u, v)$, a surface integral becomes:

$$
\iint_{S} f(u, v) d S=\int_{-\sin \theta_{0}}^{\sin \theta_{0}} \int_{-\sin \theta_{0}}^{\sin \theta_{0}} f(u, v)\left|\frac{\partial \vec{Q}}{\partial u} \times \frac{\partial \vec{Q}}{\partial v}\right| d u d v
$$




\section{A.3 Truncation angle of a $Q O$ component}

Here we discuss how to calculate the truncation angle, i.e. the subtended rim angle, for each QO component.

\section{- Parabolic reflector}

A parabolic reflector is shown in Fig. A.2, with the diameter $D_{r}$, the focal distance $f$, and the truncation angle $\theta_{0}$. The parabolic surface is defined by the parabola equation:

$$
z=f-\frac{\rho^{2}}{4 f}
$$

where $\rho=\sqrt{x^{2}+y^{2}}$. The lowest point of the surface, $z_{\text {min }}$, can be calculated by substituting $\rho=D_{r} / 2$ in Eq. (A.9):

$$
z_{\min }=f-\frac{D_{r}^{2}}{16 f}
$$

By using $D_{r}$ and $z_{\min }$, one can obtain the truncation angle as follows:

$$
\theta_{0}=\tan ^{-1}\left(\frac{D_{r}}{2 z_{\min }}\right)=2 \tan ^{-1}\left(\frac{D}{4 f}\right)
$$

\section{- Elliptical lens}

Fig. A.3 shows the 2D geometry of an elliptical lens (diameter $D_{l}$ ). Here we highlight the foci of the ellipse with the red solid dots, i.e. $O_{1}$ and $O_{2}$, and represent the center of the ellipse by the red dashed dot. The lens is defined by the ellipse equation as:

$$
\left(\frac{z-c}{a}\right)^{2}+\left(\frac{\rho}{b}\right)^{2}=1
$$

By substituting $\rho=D_{l} / 2$ in Eq. (A.12), one can obtain $z_{\text {min }}$ :

$$
z_{\text {min }}=a \sqrt{1-\left(\frac{D_{l}}{2 b}\right)^{2}}+c
$$

And then one can calculate $\theta_{0}$ as:

$$
\theta_{0}=\tan ^{-1}\left(\frac{D_{l}}{2 z_{\min }}\right)
$$

Moreover, the rim distance, $R_{l}$, can be derived by using $\theta_{0}$ :

$$
R_{l}=\frac{D_{l}}{2 \sin \theta_{0}}
$$




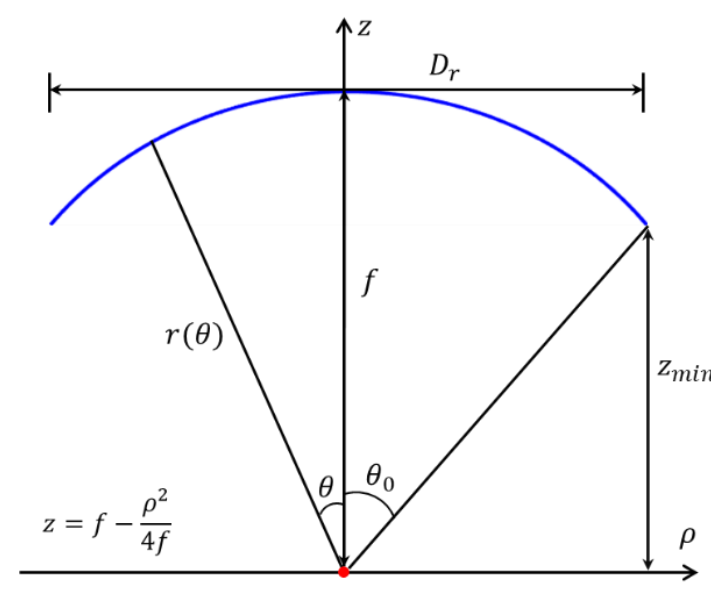

Figure A.2: 2D geometry of a parabolic reflector.

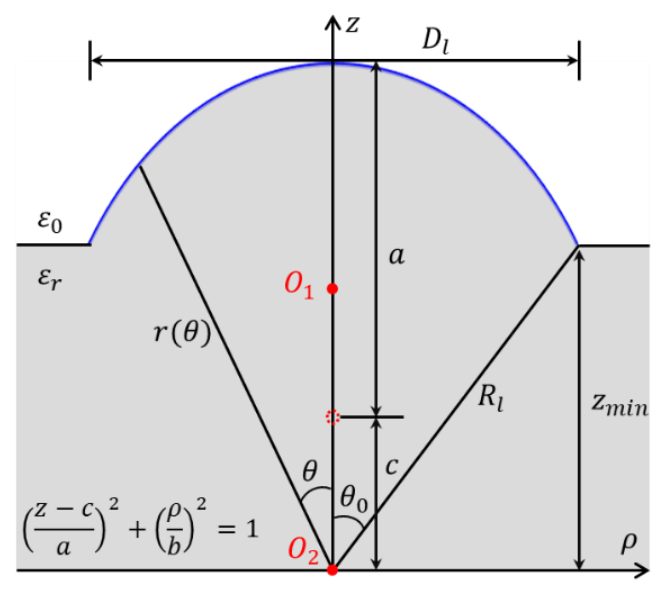

Figure A.3: 2D geometry of an elliptical lens.

\section{- Hemispherical lens}

Fig. A.4 shows the 2D geometry of a hemispherical lens which consists of a hemisphere (radius $R_{s p h}$ ) and an extended base (length $L$ ). The center of the hemisphere is represented by the red dashed dot, $O^{\prime}$. One can define the hemisphere by using the sphere equation:

$$
(z-L)^{2}+\rho^{2}=R_{s p h}^{2}
$$

The truncation angle $\theta_{0}$ is calculated as follows:

$$
\theta_{0}=\tan ^{-1}\left[\frac{D_{h l}}{2(h+L)}\right]
$$

where $h=\sqrt{R_{s p h}^{2}-\left(D_{h l} / 2\right)^{2}}$. And the rim distance, $R_{l}$, is expressed as:

$$
R_{l}=\frac{D_{h l}}{2 \sin \theta_{0}}
$$

\section{- Hyperbolic lens}

In the case of a hyperbolic lens, as shown in Fig. A.5, the center of the hyperbola is represented by the red dashed dot while the focus is represented by the red solid dot, $O$. The hyperbolic lens can be defined by using the hyperbola equation:

$$
\left(\frac{z-c}{a}\right)^{2}-\left(\frac{\rho}{b}\right)^{2}=1
$$

By substituting $\rho=D_{h} / 2$ in Eq. (A.19), one can obtain the highest point of the hyperbola, $z_{\max }$ :

$$
z_{\text {max }}=a \sqrt{1+\left(\frac{D_{h}}{2 b}\right)^{2}}+c
$$


The truncation angle $\theta_{0}$ can then be calculated as follows:

$$
\theta_{0}=\tan ^{-1}\left(\frac{D_{h}}{2 z_{\max }}\right)
$$

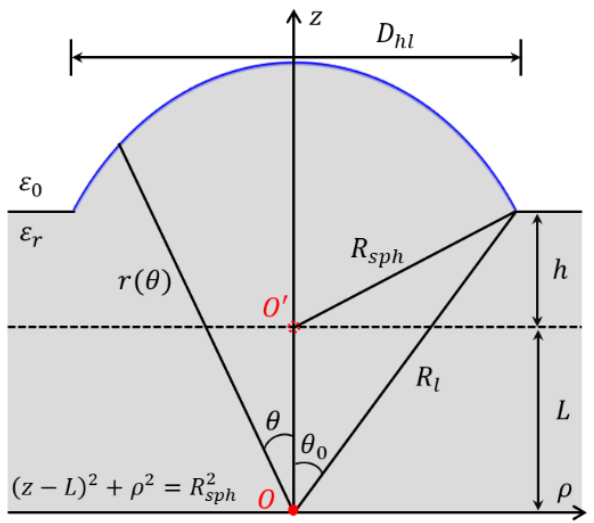

Figure A.4: 2D geometry of a hemispherical lens.

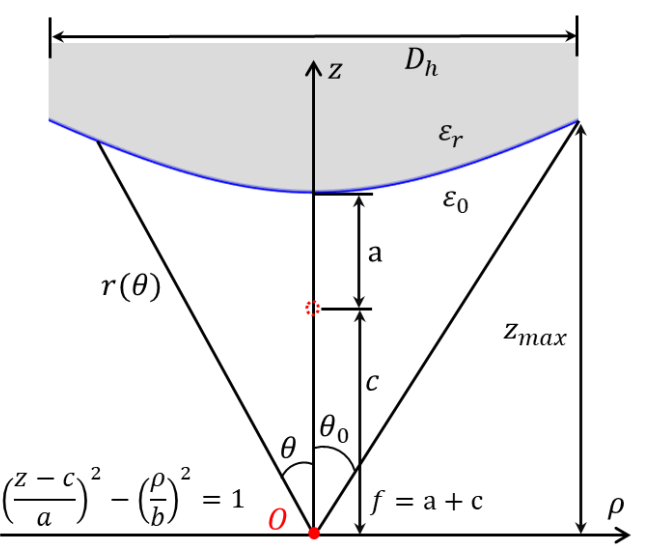

Figure A.5: 2D geometry of a hyperbolic lens.

\section{- Elliptical mirror}

In the case of an elliptical mirror, as shown in Fig. A.6, the truncation angle can be selected as $\theta_{01}$ or $\theta_{02}$, which depends on where we place the observation plane.

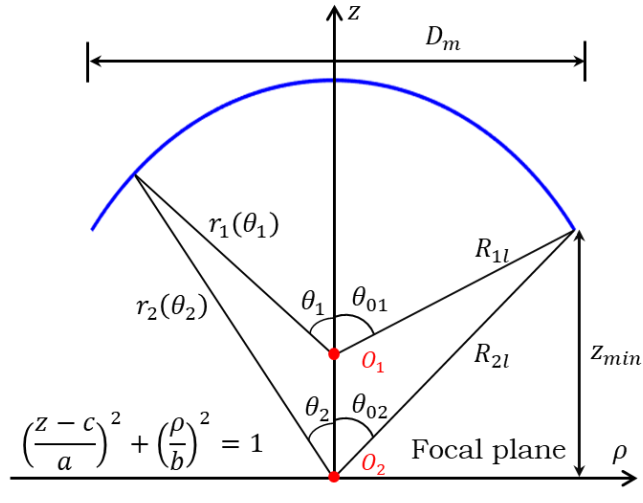

(a)

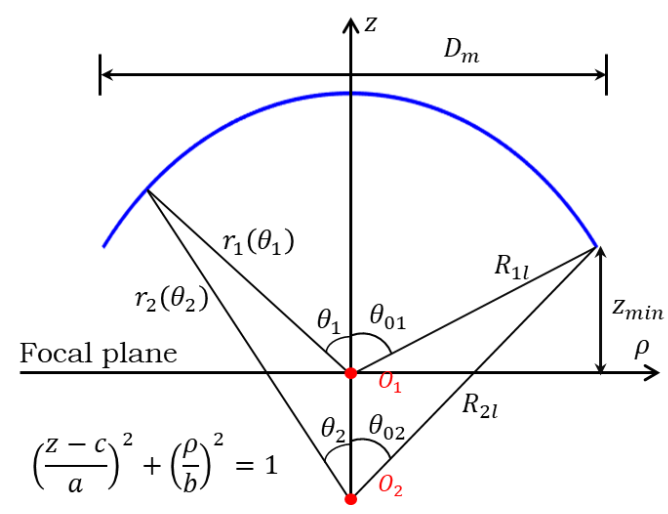

(b)

Figure A.6: 2D geometry of an elliptical mirror. (a) Observation at lower focal plane. (b) Observation at upper focal plane.

\section{Case 1: Observation at lower focal plane}

In this case, as shown in Fig. A.6a, the mirror is parameterized by the radial distance, $r_{2}$, with the variable $\left(\theta_{2}, \phi_{2}\right)$ or $\left(u_{2}, v_{2}\right)$. The lowest point of the ellipse, $z_{\min }$, is calculated by using the ellipse equation in Eq. (A.12): 


$$
z_{\text {min }}=a \sqrt{1-\left(\frac{D_{m}}{2 b}\right)^{2}}+c
$$

We define the truncation angle as $\theta_{02}$. By using $z_{\text {min }}$, one can obtain $\theta_{02}$ directly:

$$
\theta_{02}=\tan ^{-1}\left(\frac{D_{m}}{2 z_{\min }}\right)
$$

Moreover, one can calculate the rim distance, $R_{2 l}$ :

$$
R_{2 l}=\frac{D_{m}}{2 \sin \theta_{02}}
$$

The second rim angle, $\theta_{01}$, can be derived by using $\theta_{02}$ and $R_{2 l}$ :

$$
\theta_{01}=\tan ^{-1}\left[\frac{D_{m}}{2\left(R_{2 l} \cos \theta_{02}-2 c\right)}\right]
$$

And the second rim distance $R_{1 l}$ can be expressed as:

$$
R_{1 l}=\frac{D_{m}}{2 \sin \theta_{01}}
$$

\section{Case 2: Observation at upper focal plane}

In this case, as shown in Fig. A.6b, the mirror is parameterized by the radial distance, $r_{1}$, with the variable $\left(\theta_{1}, \phi_{1}\right)$ or $\left(u_{1}, v_{1}\right)$.The lowest point of the ellipse, $z_{\min }$, is calculated as:

$$
z_{\text {min }}=a \sqrt{1-\left(\frac{D_{m}}{2 b}\right)^{2}}-c
$$

We define the truncation angle as $\theta_{01}$ which can be calculated as follows:

$$
\theta_{01}=\tan ^{-1}\left(\frac{D_{m}}{2 z_{\min }}\right)
$$

$R_{1 l}$ and $R_{2 l}$ are derived in Eq. (A.26) and Eq. (A.24), respectively. And in Eq. (A.24), $\theta_{02}$ is obtained as follows:

$$
\theta_{02}=\tan ^{-1}\left[\frac{D_{m}}{2\left(R_{1 l} \cos \theta_{01}+2 c\right)}\right]
$$




\section{Appendix B Fresnel reflection and transmission coefficients}

In this appendix, we will derive Fresnel reflection and transmission coefficients explicitly for a surface with and without a matching layer, respectively.

\section{B.1 Surface without a matching layer}

When a flat interface is illuminated by an incident ray, as shown in Fig. B.1, the Fresnel transmission coefficients can be derived by imposing boundary conditions on electric and magnetic fields:

$$
\left\{\begin{array}{l}
\hat{n} \times\left.\left(\vec{E}_{1}-\vec{E}_{2}\right)\right|_{Q}=0 \\
\hat{n} \times\left.\left(\vec{H}_{1}-\vec{H}_{2}\right)\right|_{Q}=0
\end{array} \Rightarrow \begin{array}{l}
\hat{n} \times\left.\left(\vec{E}_{i}+\vec{E}_{r}-\vec{E}_{t}\right)\right|_{Q}=0 \\
\hat{n} \times\left.\left(\vec{H}_{i}+\vec{H}_{r}-\vec{H}_{t}\right)\right|_{Q}=0
\end{array}\right.
$$

By solving the boundary conditions for both TE and TM components, one can obtain the transmission coefficients:

$$
\left\{\begin{array}{l}
\tau^{\perp}(Q)=\frac{2 \zeta_{2} \cos \theta_{i}}{\zeta_{2} \cos \theta_{i}+\zeta_{1} \cos \theta_{t}} \\
\tau^{\|}(Q)=\frac{2 \zeta_{2} \cos \theta_{i}}{\zeta_{1} \cos \theta_{i}+\zeta_{2} \cos \theta_{t}}
\end{array}\right.
$$

and the reflection coefficients:

$$
\left\{\begin{array}{l}
\Gamma^{\perp}(Q)=\frac{\zeta_{2} \cos \theta_{i}-\zeta_{1} \cos \theta_{t}}{\zeta_{2} \cos \theta_{i}+\zeta_{1} \cos \theta_{t}} \\
\Gamma^{\|}(Q)=\frac{\zeta_{1} \cos \theta_{i}-\zeta_{2} \cos \theta_{t}}{\zeta_{1} \cos \theta_{i}+\zeta_{2} \cos \theta_{t}}
\end{array}\right.
$$

where $\cos \theta_{i}$ and $\cos \theta_{t}$ are derived as:

$$
\left\{\begin{array}{l}
\cos \theta_{i}=-\left(\hat{s}_{i} \cdot \hat{n}\right)=\cos \theta_{r} \\
\cos \theta_{t}=-\left(\hat{s}_{t} \cdot \hat{n}\right)
\end{array}\right.
$$

Moreover, one can derive a useful relation by using the preceding coefficients:

$$
1-\left|\Gamma^{\perp / \|}\right|^{2}=\left|\tau^{\perp / \|}\right|^{2} \frac{\zeta_{1}}{\zeta_{2}} \frac{\cos \theta_{t}}{\cos \theta_{i}}
$$

In the case of illuminating a PEC, there is no field transmitted into the PEC, i.e. $\tau^{\perp / \|}=0$. Therefore, Eq. (B.5) can be simplified:

$$
\left|\Gamma^{\perp / \|}\right|=1
$$




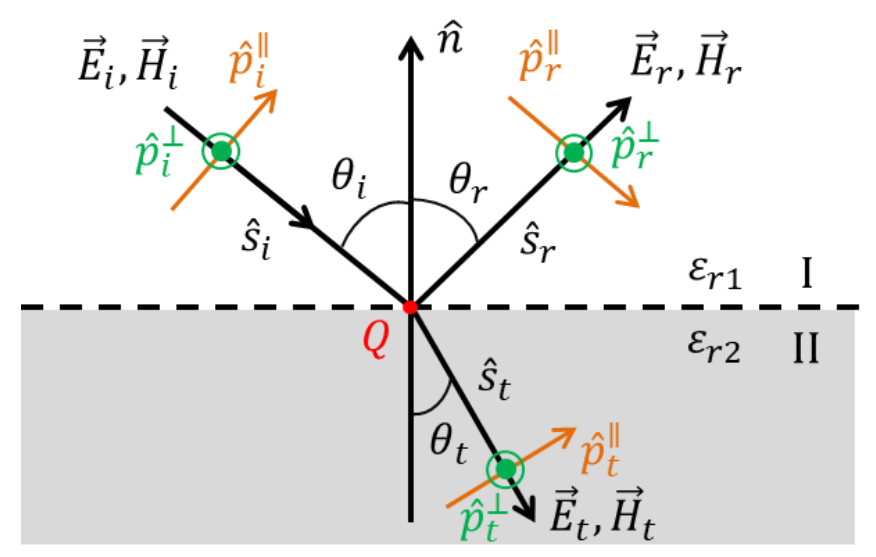

Figure B.1: Flat interface between medium I and II, illuminated by an incident ray.

\section{B.2 Surface with a matching layer}

We have introduced in section 2.2.2 that when a surface is fabricated with a matching layer, the transmission coefficients can be obtained by solving the equivalent transmission line representation, as shown in Fig. B.2.

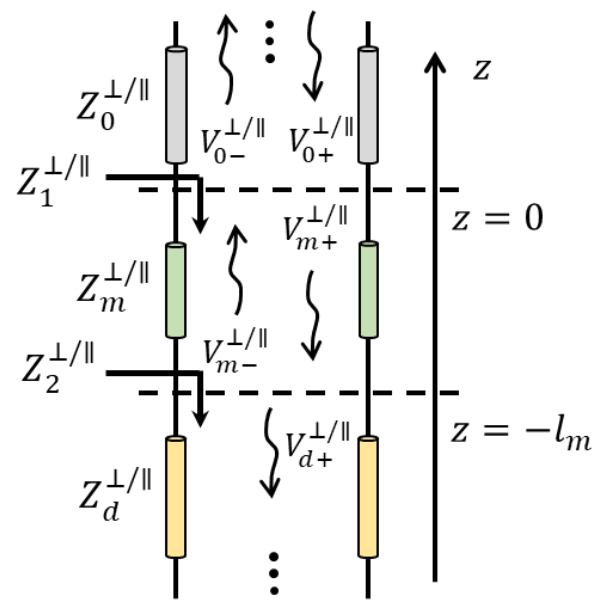

Figure. B.2: The transversal equivalent transmission line model of a dielectric stratification.

First, one needs to define the characteristic parameters of the transmission line for TE and TM components. The incident voltages can be represented by the incident fields as:

$$
\left\{\begin{array}{l}
V_{0+}^{\perp}=E_{i}^{\perp} \\
V_{0+}^{\|}=E_{i}^{\|} \cos \theta_{i}
\end{array}\right.
$$

Moreover, the propagation constants in $z$-direction are:

$$
k_{z i}=-j \sqrt{\left(k_{\rho i}\right)^{2}-\left(k_{i}\right)^{2}}, i=0, m, d
$$


where $k_{m}=k_{0} \sqrt{\varepsilon_{m}}$ and $k_{d}=k_{0} \sqrt{\varepsilon_{d}}$ are the wave numbers in the matching layer and the dielectric slab, respectively. $k_{\rho 0}=k_{0} \sin \theta_{i}, k_{\rho m}=k_{m} \sin \theta_{t}^{m}$, and $k_{\rho d}=k_{d} \sin \theta_{t}^{d}$ are the projections of the wave numbers in $\rho$-direction for air, the matching layer and the dielectric slab, respectively. By using Snell's law, one can obtain the transmitted angles: $\theta_{t}^{m}=$ $\sin ^{-1}\left(\sin \left(\theta_{i}\right) / \sqrt{\varepsilon_{m}}\right)$ and $\theta_{t}^{d}=\sin ^{-1}\left(\sin \left(\theta_{i}\right) / \sqrt{\varepsilon_{d}}\right)$. The characteristic impedances of different layers are:

$$
\left\{\begin{array}{l}
Z_{i}^{\perp}=\zeta_{i} \frac{k_{i}}{k_{z i}} \\
Z_{i}^{\|}=\zeta_{i} \frac{k_{z i}}{k_{i}}
\end{array}, i=0, m, d\right.
$$

where $\zeta_{m}=\zeta_{0} / \sqrt{\varepsilon_{m}}$, and $\zeta_{d}=\zeta_{0} / \sqrt{\varepsilon_{d}}$ are the impedances of the matching layer and the dielectric slab, respectively. Next, one is able to calculate the reflection and transmission coefficients for voltages at $z=0$ and $z=-l_{m}$ :

$$
\left\{\begin{array}{l}
\Gamma_{1}^{\perp / \|}=\frac{V_{0-}^{\perp / \|}(z=0)}{V_{0+}^{\perp / \|}(z=0)}=\frac{Z_{1}^{\perp / \|}-Z_{0}^{\perp / \|}}{Z_{1}^{\perp / \|}+Z_{0}^{\perp / \|}} \\
\Gamma_{2}^{\perp / \|}=\frac{V_{m-}^{\perp / \|}\left(z=-l_{m}\right)}{V_{m+}^{\perp / \|}\left(z=-l_{m}\right)}=\frac{z_{2}^{\perp / \|}-Z_{m}^{\perp / \|}}{Z_{2}^{\perp / \|}+Z_{m}^{\perp / \|}} \\
\tau_{1}^{\perp / \|}=\frac{V_{m}^{\perp / \|}(z=0)}{V_{0+}^{\perp / \|}(z=0)}=\frac{2 Z_{1}^{\perp / \|}}{Z_{1}^{\perp / \|}+Z_{0}^{\perp / \|}} \\
\tau_{2}^{\perp / \|}=\frac{V_{d+}^{\perp / \|}\left(z=-l_{m}\right)}{V_{m+}^{\perp / \|}\left(z=-l_{m}\right)}=\frac{2 Z_{2}^{\perp / \|}}{Z_{2}^{\perp / \|}+Z_{m}^{\perp / \|}}
\end{array}\right.
$$

where $z_{1}^{\perp / \|}$ and $z_{2}^{\perp / \|}$ are input impedances seen from $z=0$ and $z=-l_{m}$, respectively:

$$
\left\{\begin{array}{l}
Z_{2}^{\perp / \|}=Z_{d}^{\perp / \|} \\
Z_{1}^{\perp / \|}=Z_{m}^{\perp / \|} \frac{Z_{2}^{\perp / \|}+j Z_{m}^{\perp / \|} \tan \left(k_{z m} l_{m}\right)}{Z_{m}^{\perp / \|}+j Z_{2}^{\perp / \|} \tan \left(k_{z m} l_{m}\right)}
\end{array}\right.
$$

From Eq. (B.10), one can find the condition for zero reflection:

$$
\Gamma_{1}^{\perp / \|}=0 \Rightarrow Z_{1}^{\perp / \|}=Z_{0}^{\perp / \|} \Rightarrow\left\{\begin{array}{c}
\varepsilon_{m}=\sqrt{\varepsilon_{r}} \\
l_{m}=\frac{\lambda_{m}}{4}
\end{array}\right.
$$

Moreover, by using Eq. (B.10), one can calculate the progressive voltage in the matching layer:

$$
\begin{gathered}
V_{m}^{\perp / \|}(z=0)=V_{m+}^{\perp / \|}(z=0)+V_{m-}^{\perp / \|}(z=0)=\tau_{1}^{\perp / \|} V_{0+}^{\perp / \|}(z=0) \\
\Rightarrow V_{m+}^{\perp / \|}(z=0)=\frac{\tau_{1}^{\perp / \|} V_{0+}^{\perp / \|}(z=0)}{\left[1+\Gamma_{2}^{\perp / \|} e^{-2 j k_{z m} l_{m}}\right]}
\end{gathered}
$$


and the voltage in the dielectric slab:

$$
V_{d+}^{\perp / \|}\left(z=-l_{m}\right)=\tau_{2}^{\perp / \|} V_{m+}^{\perp / \|}\left(z=-l_{m}\right)=\frac{\tau_{1}^{\perp / \|} \tau_{2}^{\perp / \|} V_{0+}^{\perp / \|}(z=0) e^{-j k_{z m} l_{m}}}{\left[1+\Gamma_{2}^{\perp / \|} e^{-2 j k_{z m} l_{m}}\right]}
$$

Finally, by using Eq. (B.14), one can obtain the transmission coefficients of the stratification:

$$
\left\{\begin{array}{l}
\tau^{\perp}=\frac{E_{d}^{\perp}}{E_{i}^{\perp}}=\frac{V_{d+}^{\perp}\left(z=-l_{m}\right)}{V_{0+}^{\perp}(z=0)} \\
\tau^{\|}=\frac{E_{d}^{\|}}{E_{i}^{\|}}=\frac{V_{d+}^{\|}\left(z=-l_{m}\right)}{V_{0+}^{\|}(z=0)} \frac{\cos \theta_{i}}{\cos \theta_{t}^{d}}
\end{array}\right.
$$

\section{Appendix C Additional validation of the implemented PO analysis}

In this appendix, additional validation of the implemented PO analysis is introduced.

\section{C.1 Parabolic reflector}

Fig. C.1 shows the x-component of the electric fields on the focal plane of a parabolic reflector with the diameter of $D_{r}=100 \lambda_{0}\left(f_{0}=300 \mathrm{GHz}\right)$ and the f-number of $f_{\#}^{r}=0.6$. The reflector is illuminated by a unitary TM polarized plane wave with the skew angle of $\theta_{s}=$ $5\left(\lambda_{0} / D_{r}\right)=2.86^{\circ}, \phi_{s}=0^{\circ}$. The focal plane field evaluated by resorting to the PO analysis is compared with the GRASP simulation. The setup of the simulation in GRASP is plotted in the inset. It can be seen in the figure that the PO results are well validated for both the amplitude and the phase.

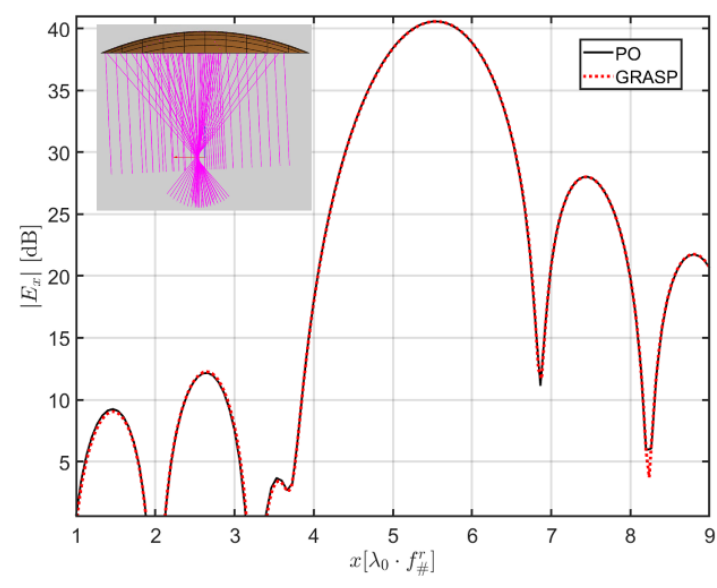

(a)

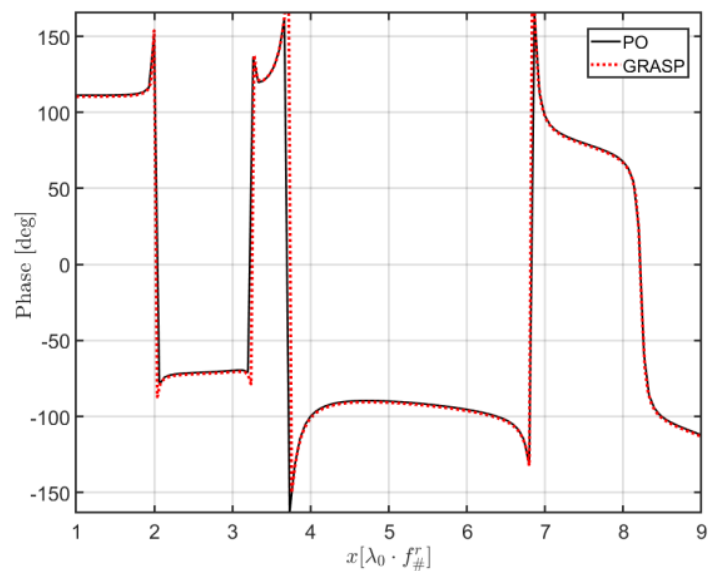

(b)

Figure C.1: The x-component of the electric fields on the focal plane of a parabolic reflector with $D_{r}=$ $100 \lambda_{0}\left(f_{0}=300 \mathrm{GHz}\right)$ and $f_{\#}^{r}=0.6$, illuminated by a unitary TM polarized plane wave with the skew angle of $\theta_{s}=5\left(\lambda_{0} / D_{r}\right)=2.86^{\circ}, \phi_{s}=0^{\circ}$. The focal plane field calculated by using the PO analysis is compared with the GRASP simulation: (a) Amplitude. (b) Phase. One of the main planes $(y=0)$ is shown. Inset is the configuration in GRASP. 


\section{C.2 Elliptical lens}

\section{- TM polarized plane wave}

Here we validate an elliptical lens without a matching layer. Fig. C.2 shows the x-component of the electric fields on the focal plane of an elliptical silicon $\left(\varepsilon_{r}=11.9\right)$ lens with $D_{l}=5 \lambda_{0}$ $\left(f_{0}=300 \mathrm{GHz}\right)$ and $f_{\#}^{l}=0.6$. The lens is illuminated by a unitary TM polarized plane wave with the skew angle of $\theta_{S}=20^{\circ}, \phi_{S}=0^{\circ}$. And the focal plane field calculated by using the PO analysis is compared with the CST simulation. The setup of the simulation in CST is plotted in the inset. As it can be seen in the figure, the PO analysis is in fair agreement with the CST simulation, for both the amplitude and the phase.

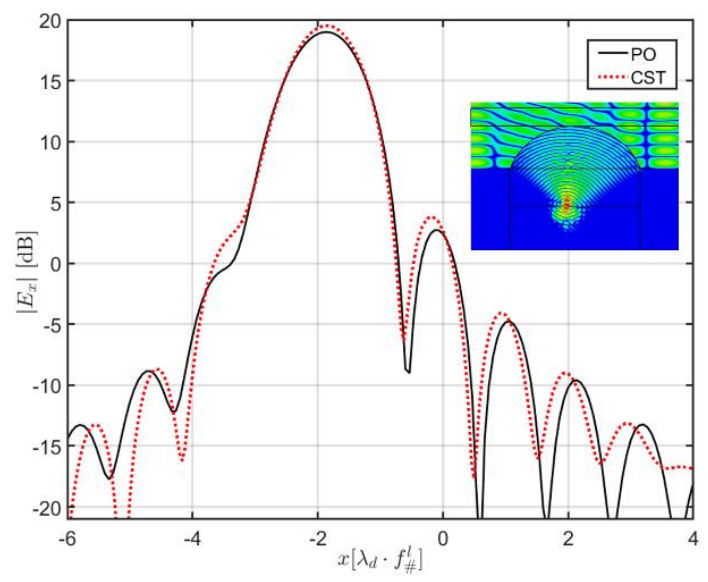

(a)

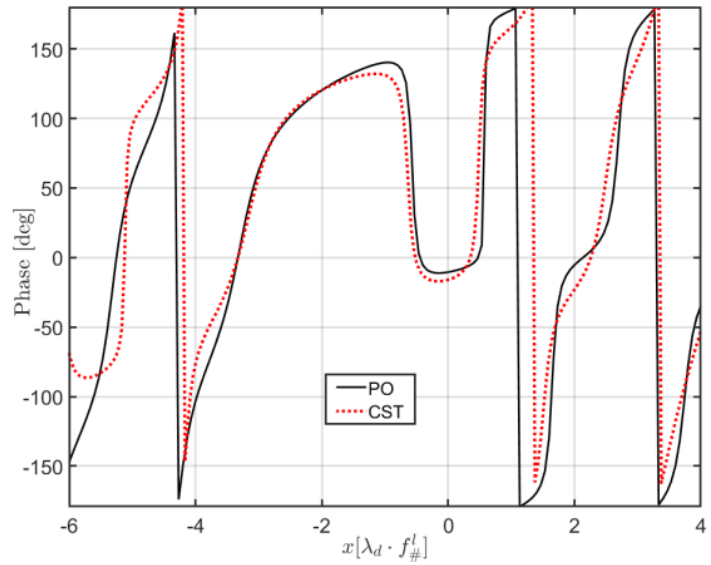

(b)

Figure C.2: The x-component of the electric fields on the focal plane of an elliptical silicon $\left(\varepsilon_{r}=11.9\right)$ lens with $D_{l}=5 \lambda_{0}\left(f_{0}=300 \mathrm{GHz}\right)$ and $f_{\#}^{l}=0.6$, illuminated by a unitary TM polarized plane wave with the skew angle of $\theta_{s}=20^{\circ}, \phi_{s}=0^{\circ}$. The focal plane field calculated by using the PO analysis is compared with the CST simulation: (a) Amplitude. (b) Phase. One of the main planes $(y=0)$ is shown. Inset is the configuration in CST.

\section{- TE polarized plane wave}

For the same elliptical lens, when the plane wave is TE polarized with the skew angle of $\theta_{s}=$ $20^{\circ}, \phi_{s}=0^{\circ}$, the y-component of the electric field on the focal plane is shown in Fig. C.3. As it can be seen, the PO analysis is in fair agreement with the CST simulation. 


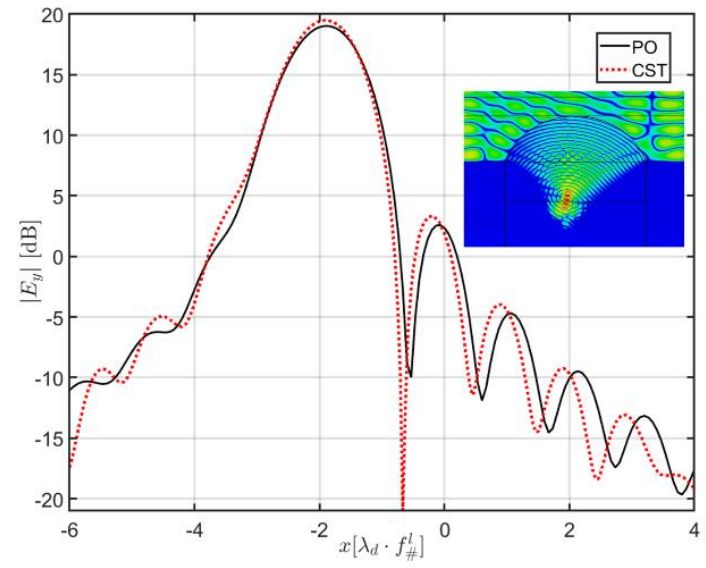

(a)

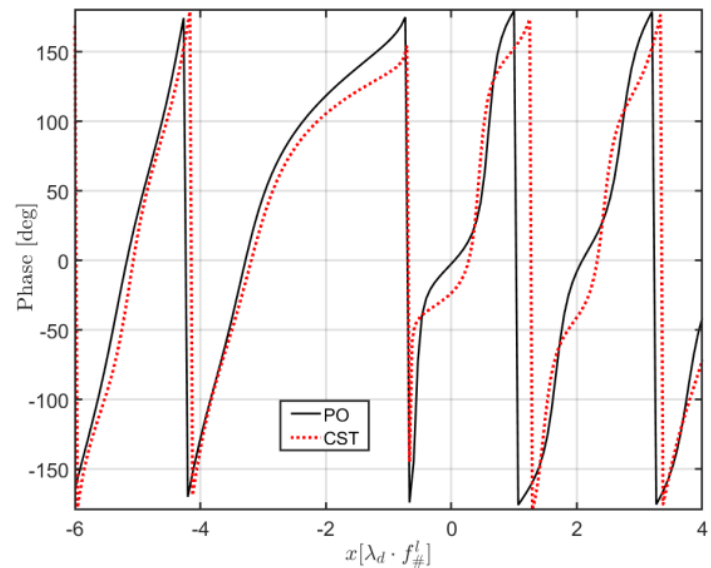

(b)

Figure C.3: The y-component of the electric fields on the focal plane of an elliptical silicon $\left(\varepsilon_{r}=11.9\right)$ lens with $D_{l}=5 \lambda_{0}\left(f_{0}=300 \mathrm{GHz}\right)$ and $f_{\#}^{l}=0.6$, illuminated by a unitary TE polarized plane wave with the skew angle of $\theta_{s}=20^{\circ}, \phi_{s}=0^{\circ}$. The focal plane field calculated by using the PO analysis is compared with the CST simulation: (a) Amplitude. (b) Phase. One of the main planes $(y=0)$ is shown. Inset is the configuration in CST.

\section{C.3 Hemispherical lens}

Fig. C.4 shows the y-component of the electric fields on the focal plane of a hemispherical silicon $\left(\varepsilon_{r}=11.9\right)$ lens with $D_{h l}=5 \lambda_{0}\left(f_{0}=300 \mathrm{GHz}\right), f_{\#}^{h l}=0.74, R_{s p h}=3 \lambda_{0}$, and $L=$ $0.362 R_{s p h}$. The lens is illuminated by a unitary TE polarized plane wave with the skew angle of $\theta_{s}=20^{\circ}, \phi_{s}=0^{\circ}$. The focal plane field evaluated by using the PO analysis is compared with the CST simulation. As it can be seen, the PO results are validated for both the amplitude and the phase.

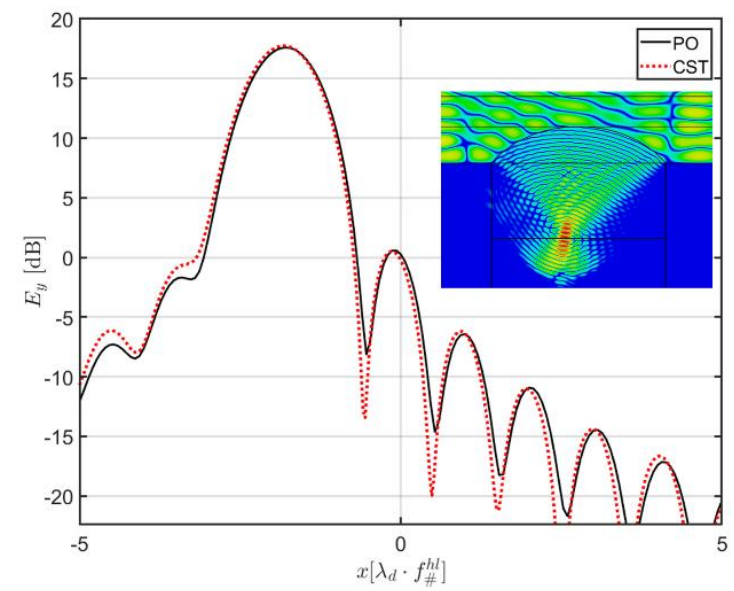

(a)

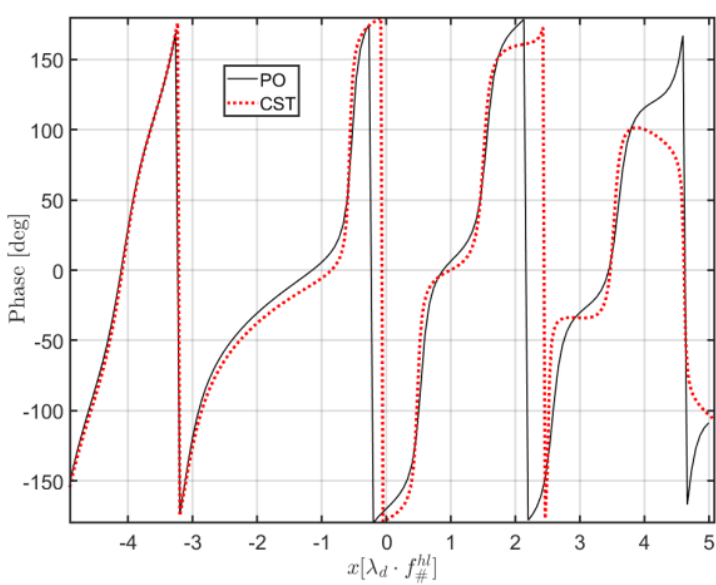

(b)

Figure C.4: The y-component of the electric fields on the focal plane of a hemispherical silicon $\left(\varepsilon_{r}=11.9\right)$ lens with $D_{h l}=5 \lambda_{0}\left(f_{0}=300 \mathrm{GHz}\right), f_{\#}^{h l}=0.74, R_{s p h}=3 \lambda_{0}$, and $L=0.362 R_{s p h}$, illuminated by a unitary TE polarized plane wave with the skew angle of $\theta_{s}=20^{\circ}, \phi_{s}=0^{\circ}$. The focal plane field calculated by using the PO analysis is compared with the CST simulation: (a) Amplitude. (b) Phase. One of the main planes $(y=0)$ is shown. Inset is the configuration in CST. 


\section{C.4 Elliptical mirror}

In the case of an elliptical mirror, a source can be placed at either its upper focal plane or its lower focal plane. Here we consider a mirror with the diameter of $D_{m}=500 \lambda_{0}\left(f_{0}=\right.$ $300 \mathrm{GHz}$ ), the semi-major axis of $a=278 \lambda_{0}$, and the focal distance of $c=55.6 \lambda_{0}$, i.e. the eccentricity is $e=0.2$.

\section{Case 1: Source placed at the upper focal plane}

In this case, the $\mathrm{f}$-number is calculated as $f_{\#}^{m}=0.6$. A unitary Huygens source is placed at the upper focal plane, with the electric current oriented along $\hat{y}$ and a displacement in y-direction, $y_{s}=4 \lambda_{0} f_{\#}^{m}$. Fig. C.5 shows the y-component of the electric fields on the focal plane of the mirror. The focal plane field obtained by using the PO analysis is compared with the GRASP simulation. As it can be seen, the PO code is well validated.

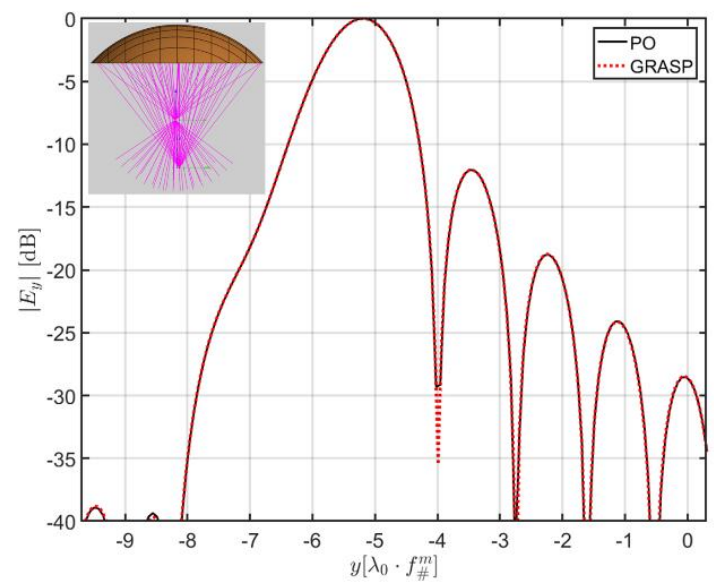

(a)

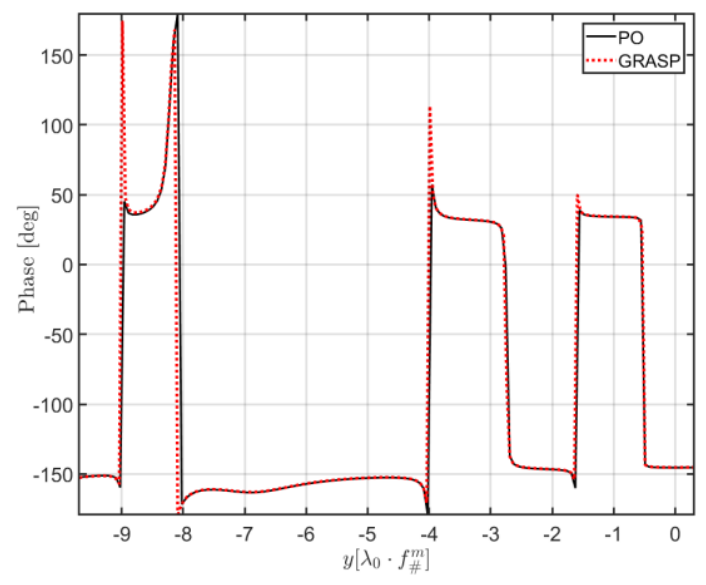

(b)

Figure C.5: The y-component of the electric fields on the focal plane of an elliptical mirror with $D_{m}=$ $500 \lambda_{0}\left(f_{0}=300 \mathrm{GHz}\right), f_{\#}^{m}=0.6$ and $e=0.2$. The mirror is illuminated by a unitary Huygens source placed at the upper focal plane, with the electric current oriented along $\hat{y}$ and a displacement in y-direction, $y_{s}=4 \lambda_{0} f_{\#}^{m}$. The focal plane field evaluated by using the PO analysis is compared with the GRASP simulation: (a) Amplitude. (b) Phase. One of the main planes $(x=0)$ is shown. Inset is the configuration in GRASP. 


\section{Case 2: Source placed at the lower focal plane}

In this case, the $\mathrm{f}$-number is obtained as $f_{\#}^{m}=0.44$. The Huygens source is placed at the lower focal plane, with the electric current oriented along $\hat{x}$ and a displacement in $\mathrm{x}$-direction, $x_{s}=$ $10 \lambda_{0} f_{\#}^{m}$. Fig. C.6 shows the x-component of the electric fields on the focal plane of the mirror. The focal plane field obtained by using the PO analysis is compared with the GRASP simulation. It can be seen that the PO code is in excellent agreement with the GRASP simulation.

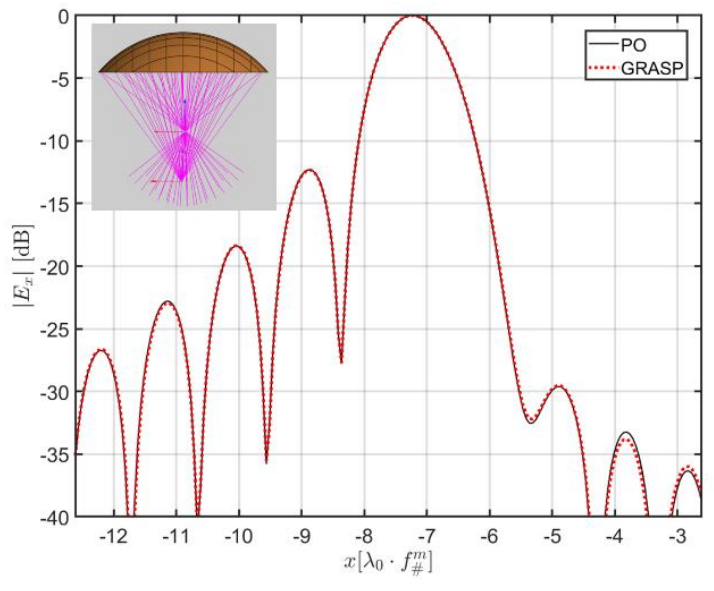

(a)

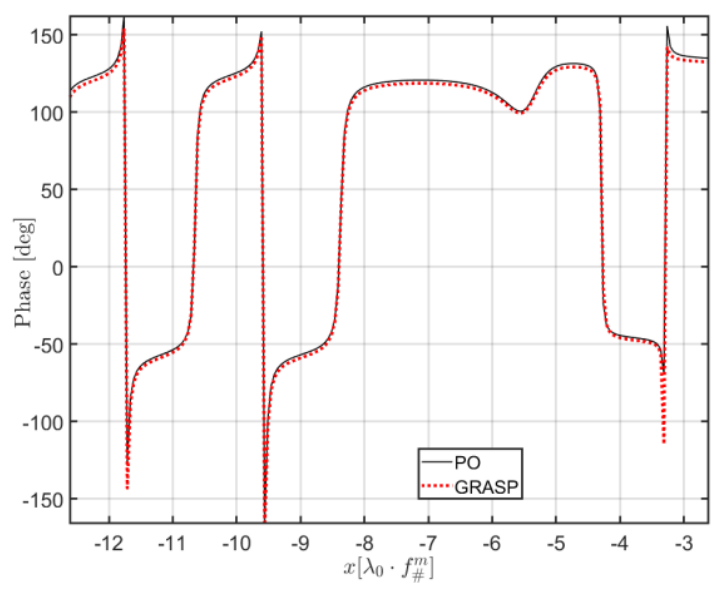

(b)

Figure C.6: The $\mathrm{x}$-component of the electric fields on the focal plane of an elliptical mirror with $D_{m}=$ $500 \lambda_{0}\left(f_{0}=300 \mathrm{GHz}\right), f_{\#}^{m}=0.44$ and $e=0.2$. The mirror is illuminated by a unitary Huygens source placed at the lower focal plane, with the electric current oriented along $\hat{x}$ and a displacement in $\mathrm{x}$-direction, $x_{s}=$ $10 \lambda_{0} f_{\#}^{m}$. The focal plane field evaluated by using the PO analysis is compared with the GRASP simulation: (a) Amplitude. (b) Phase. One of the main planes $(y=0)$ is shown. Inset is the configuration in GRASP. 


\section{Appendix D Re-evaluate the phase approximation for the FO applicability region}

In Eq. (3.8), the condition for the FO phase approximation is determined by neglecting the phase term $e^{-j k \frac{\rho_{f}^{2}}{2 R_{F O}}}$ when setting a phase error of $\pi / 8$. This condition is valid for QO components with relatively small $f_{\#}$ but not for cases with large $f_{\#}$. Therefore, here we discuss a more general condition for the phase approximation. The term $\left|\vec{\rho}_{f}-\vec{r}^{\prime}\right|$, can be expanded as follows:

$$
\left|\vec{\rho}_{f}-\vec{r}^{\prime}\right|=\sqrt{\left(\vec{\rho}_{f}-\vec{r}^{\prime}\right) \cdot\left(\vec{\rho}_{f}-\vec{r}^{\prime}\right)}=R_{F O} \sqrt{1-\frac{2}{R_{F O}}\left(\vec{\rho}_{f} \cdot \hat{r}^{\prime}\right)+\frac{\rho_{f}^{2}}{R_{F O}^{2}}}
$$

where $R_{F O}=\left|\vec{r}^{\prime}\right|$ and $\rho_{f}=\left|\vec{\rho}_{f}\right|$. Eq. (D.1) can be approximated by expanding the square root for small argument to the second order $\left(\sqrt{1+x} \simeq 1+\frac{x}{2}-\frac{x^{2}}{8}+\cdots\right)$ :

$$
\left|\vec{\rho}_{f}-\vec{r}^{\prime}\right| \approx R_{F O}-\vec{\rho}_{f} \cdot \hat{r}^{\prime}+\frac{\rho_{f}^{2}}{2 R_{F O}}\left[1-\left(\hat{\rho}_{f} \cdot \hat{r}^{\prime}\right)^{2}\right]+\frac{\rho_{f}^{3}}{2 R_{F O}^{2}}\left(\hat{\rho}_{f} \cdot \hat{r}^{\prime}\right)
$$

In Eq. (D.2), the terms with order four and higher, with respect to $\rho_{f}$, are neglected. To obtain the phase approximation, one must neglect the second- and the third- order terms in Eq. (D.2) with a phase error $\sigma_{p h}$ :

$$
-k\left(-\frac{\rho_{f}^{2}}{2 R_{F O}}\left(\hat{\rho}_{f} \cdot \hat{r}^{\prime}\right)^{2}+\frac{\rho_{f}^{3}}{2 R_{F O}^{2}}\left(\hat{\rho}_{f} \cdot \hat{r}^{\prime}\right)\right) \leq \sigma_{p h}
$$

In practice, we choose $\sigma_{p h}=\pi / 8$. The worst case in Eq. (D.3) happens when $\hat{\rho}_{f} \cdot \hat{r}^{\prime}=$ $-\sin \theta_{0} \approx-1 / 2 f_{\#}$. By using this, Eq. (D.3) can be expressed as below:

$$
\frac{4 \rho_{f}^{3} D}{R_{F O}^{3}}+\frac{2 \rho_{f}^{2} D}{R_{F O}^{3}}-\lambda \leq 0
$$

By solving the cubic equation of Eq. (D.4) and taking the positive and real solution, $\rho_{p h}^{F O}$, the new condition for the phase approximation can be expressed as follows:

$$
\rho_{f} \leq \rho_{p h}^{F O}
$$




\section{Appendix E Characteristic parameters used in GO ray fields}

In this appendix, we will discuss the characteristic parameters mentioned in section 4.1. First, we consider evaluating the distance along the ray path, and then the principal radii of curvature for reflected and transmitted rays, respectively. Finally, we describe a specific surface of revolution, by deriving its principal directions and principal radii of curvature analytically.

\section{E.1 Distance along the ray path}

In the GO method, one key parameter related to represent the scattered fields is the distance propagated along the ray path, $s$. In Fig. E.1, we use a reflection problem as an example to show how we define this distance.

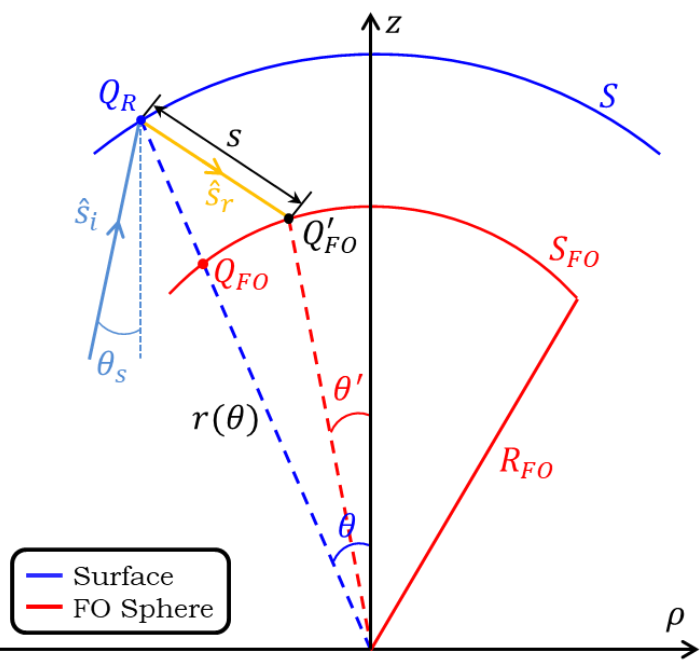

Figure E.1: Geometry for a reflection problem with the description of the distance along the ray path.

It can be observed in the figure that the reflected ray propagates from $Q_{R}(\theta, \phi)$ to $Q_{F O}\left(\theta^{\prime}, \phi^{\prime}\right)$. The distance $s$ can be calculated by solving the following equations in $(\theta, \phi)$ variable:

$$
\left\{\begin{array}{l}
Q_{x}+s k_{r, x}=R_{F O} \sin \theta^{\prime} \cos \phi^{\prime} \\
Q_{y}+s k_{r, y}=R_{F O} \sin \theta^{\prime} \sin \phi^{\prime} \\
Q_{z}+s k_{r, z}=R_{F O} \cos \theta^{\prime}
\end{array}\right.
$$

or in $(u, v)$ variable:

$$
\left\{\begin{array}{l}
Q_{x}+s k_{r, x}=R_{F O} u^{\prime} \\
Q_{y}+s k_{r, y}=R_{F O} v^{\prime} \\
Q_{z}+s k_{r, z}=R_{F O} \sqrt{1-\left(u^{\prime 2}+v^{\prime 2}\right)}
\end{array}\right.
$$


where $Q_{R}=\left(Q_{x}, Q_{y}, Q_{z}\right)$ is a point on the surface $S, \hat{s}_{r}=k_{r, x} \hat{x}+k_{r, y} \hat{y}+k_{r, z} \hat{z}$ is the propagation unit vector of the reflected ray, and $\left(\theta^{\prime}, \phi^{\prime}\right)$ and $\left(u^{\prime}, v^{\prime}\right)$ are non-uniform variables that parameterize a point on the FO sphere that is hit by the reflected ray. By solving Eq. (E.1) or (E.2), the distance $s$ can be expressed as:

$$
s=\frac{-b-\sqrt{b^{2}-4 c}}{2}
$$

where $b=2 Q_{x} k_{r / t, x}+2 Q_{y} k_{r / t, y}+2 Q_{z} k_{r / t, z}$, and $c=Q_{x}^{2}+Q_{y}^{2}+Q_{z}^{2}-R_{F O}^{2}$. After obtaining $s$, one can also calculate the non-uniform variables, $\left(\theta^{\prime}, \phi^{\prime}\right)$, by solving Eq. (E.1):

$$
\left\{\begin{array}{l}
\theta^{\prime}=\cos ^{-1}\left(\frac{Q_{z}+s k_{r, z}}{R_{F O}}\right) \\
\phi^{\prime}=\tan ^{-1}\left(\frac{Q_{y}+s k_{r, y}}{Q_{x}+s k_{r, x}}\right)
\end{array}\right.
$$

while $\left(u^{\prime}, v^{\prime}\right)$ are derived by solving Eq. (E.2):

$$
\left\{\begin{array}{l}
u^{\prime}=\frac{Q_{x}+s k_{r, x}}{R_{F O}} \\
v^{\prime}=\frac{Q_{y}+s k_{r, y}}{R_{F O}}
\end{array}\right.
$$

The preceding derivations are also applicable for a transmission problem but with a single change: one should replace the reflected propagation unit vector $\hat{s}_{r}$ by the transmitted one: $\hat{s}_{t}=$ $k_{t, x} \hat{x}+k_{t, y} \hat{y}+k_{t, z} \hat{z}$.

\section{E.2 Principal radii of curvature of a reflected wave front in a reflection}

\section{problem}

Fig. E. 2 shows a scenario that an incident ray impinges on a surface $S$ at $Q_{R}$ and reflected. In the figure, $\widehat{U}_{1}$ and $\widehat{U}_{2}$ are the unit vectors in the principal directions of $S$ at $Q_{R}$ with the principal radii of curvature $R_{1}$ and $R_{2}$, respectively. $\hat{X}_{1}^{i}$ and $\hat{X}_{2}^{i}$ are the principal directions of the incident wave front at $Q_{R}$ with the principal radii of curvature $\rho_{1}^{i}$ and $\rho_{2}^{i}$. While $\hat{X}_{1}^{r}$ and $\hat{X}_{2}^{r}$ are the principal directions of the reflected wave front at $Q_{R}$ with the principal radii of curvature $\rho_{1}^{r}$ and $\rho_{2}^{r}$. Notice that $\hat{x}_{1}^{r}$ and $\hat{x}_{2}^{r}$ do not point towards the principal directions of the reflected wave front. They are unit vectors perpendicular to $\hat{s}_{r}$, which can be calculated by Snell's law:

$$
\hat{x}_{1,2}^{r}=\hat{X}_{1,2}^{i}-2\left(\hat{n} \cdot \hat{X}_{1,2}^{i}\right) \hat{n}
$$

where $\hat{n}$ is the normal vector of the surface. 


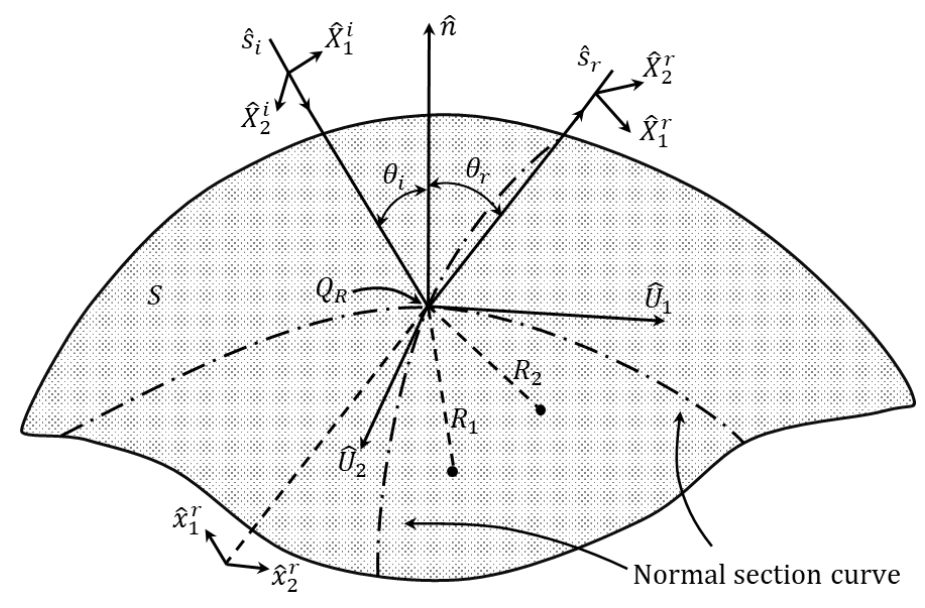

Figure E.2: Geometry for a reflection problem with the description of the curved surface $S$, incident ray and reflected ray.

\section{- Principal radii of curvature of the incident wave front}

We have introduced two types of incident waves in this thesis: a plane wave and a spherical wave. For a plane wave incidence, the incident wave front is planar, so the principal radii of curvature are: $\rho_{1}^{i}=\rho_{2}^{i}=\infty$. For a spherical wave, the wave front is spherical; therefore, the principal radii of curvature are: $\rho_{1}^{i}=\rho_{2}^{i}=r$, where $r$ is the radial distance from the source to the surface. As for the principal directions of the incident wave front, $\hat{X}_{1}^{i}$ and $\hat{X}_{2}^{i}$ can be defined arbitrarily, as long as they keep the following relation:

$$
\hat{X}_{1}^{i} \perp \hat{s}_{i}, \hat{X}_{2}^{i} \perp \hat{s}_{i}, \hat{X}_{2}^{i} \perp \hat{X}_{1}^{i}
$$

\section{- Principal radii of curvature of the reflected wave front}

To calculate GO fields, one also needs to calculate the principal radii of curvature of the reflected wave front, $\rho_{1}^{r}$ and $\rho_{2}^{r}$. First, we introduce a curvature matrix $Q^{r}$ for the reflected wave front, as described in [43]:

$$
Q^{r}=\left[\begin{array}{ll}
Q_{11}^{r} & Q_{12}^{r} \\
Q_{12}^{r} & Q_{22}^{r}
\end{array}\right]
$$

whose entries are calculated as follows:

$$
\left\{\begin{array}{l}
Q_{11}^{r}=\frac{1}{\rho_{1}^{i}}+\frac{2 \cos \theta_{i}}{|\Theta|^{2}}\left[\frac{\left(\Theta_{22}\right)^{2}}{R_{1}}+\frac{\left(\Theta_{21}\right)^{2}}{R_{2}}\right] \\
Q_{12}^{r}=-\frac{2 \cos \theta_{i}}{|\Theta|^{2}}\left[\frac{\Theta_{22} \Theta_{12}}{R_{1}}+\frac{\Theta_{11} \Theta_{21}}{R_{2}}\right] \\
Q_{22}^{r}=\frac{1}{\rho_{2}^{i}}+\frac{2 \cos \theta_{i}}{|\Theta|^{2}}\left[\frac{\left(\Theta_{12}\right)^{2}}{R_{1}}+\frac{\left(\Theta_{11}\right)^{2}}{R_{2}}\right]
\end{array}\right.
$$


where $\Theta$ is also a matrix that can be expressed as:

$$
\Theta=\left[\begin{array}{ll}
\Theta_{11} & \Theta_{12} \\
\Theta_{21} & \Theta_{22}
\end{array}\right]=\left[\begin{array}{ll}
\widehat{X}_{1}^{i} \cdot \widehat{U}_{1} & \widehat{X}_{1}^{i} \cdot \widehat{U}_{2} \\
\widehat{X}_{2}^{i} \cdot \widehat{U}_{1} & \widehat{X}_{2}^{i} \cdot \widehat{U}_{2}
\end{array}\right]
$$

And $|\Theta|$ is the determinant of $\Theta$ :

$$
|\Theta|=\left(\widehat{X}_{1}^{i} \cdot \widehat{U}_{1}\right)\left(\widehat{X}_{2}^{i} \cdot \widehat{U}_{2}\right)-\left(\widehat{X}_{1}^{i} \cdot \widehat{U}_{2}\right)\left(\widehat{X}_{2}^{i} \cdot \widehat{U}_{1}\right)
$$

By using the curvature matrix $Q^{r}$, one could obtain $\rho_{1}^{r}$ and $\rho_{2}^{r}$ :

$$
\left\{\begin{array}{l}
\frac{1}{\rho_{1}^{r}}=\frac{1}{2}\left\{Q_{11}^{r}+Q_{22}^{r}+\left[\left(Q_{11}^{r}-Q_{22}^{r}\right)^{2}+4\left(Q_{12}^{r}\right)^{2}\right]^{\frac{1}{2}}\right\} \\
\frac{1}{\rho_{2}^{r}}=\frac{1}{2}\left\{Q_{11}^{r}+Q_{22}^{r}-\left[\left(Q_{11}^{r}-Q_{22}^{r}\right)^{2}+4\left(Q_{12}^{r}\right)^{2}\right]^{\frac{1}{2}}\right\}
\end{array}\right.
$$

Notice that $\widehat{U}_{1}, \widehat{U}_{2}, R_{1}$ and $R_{2}$ are derived for a specific surface of revolution in Appendix E.4. As for the principal directions of the reflected wave front, $\hat{X}_{1}^{r}$ and $\hat{X}_{2}^{r}$, they are not used in this work, but they are useful information when we use a cascade system like multiple reflectors. $\hat{X}_{1}^{r}$ and $\hat{X}_{2}^{r}$ can be calculated by using $\hat{x}_{1}^{r}$ and $\hat{x}_{2}^{r}$ as below:

$$
\left\{\begin{array}{l}
\hat{X}_{1}^{r}=\frac{\left[\left(Q_{22}^{r}-1 / \rho_{1}^{r}\right) \hat{x}_{1}^{r}-Q_{12}^{r} \hat{x}_{2}^{r}\right]}{\sqrt{\left(Q_{22}^{r}-1 / \rho_{1}^{r}\right)^{2}+\left(Q_{12}^{r}\right)^{2}}} \\
\hat{X}_{2}^{r}=-\hat{s}_{r} \times \hat{X}_{1}^{r}
\end{array}\right.
$$




\section{E.3 Principal radii of curvature of a transmitted wave front in a transmission problem}

Fig. E. 3 describes a scenario that an incident ray propagating in medium I impinges on a surface $S$ at $Q_{T}$, and transmitted into medium II. The first and second mediums have the refraction indexes of $n_{i}=\sqrt{\varepsilon_{r 1}}$ and $n_{t}=\sqrt{\varepsilon_{r 2}}$, respectively. In the figure, $\widehat{U}_{1} \widehat{U}_{2}, R_{1}, R_{2}, \widehat{X}_{1}^{i}$, and $\widehat{X}_{2}^{i}$ are already introduced in Appendix E.2. $\hat{X}_{1}^{t}$ and $\hat{X}_{2}^{t}$ are the principal directions of the transmitted wave front at $Q_{T}$ with the principal radii of curvature $\rho_{1}^{t}$ and $\rho_{2}^{t}$.

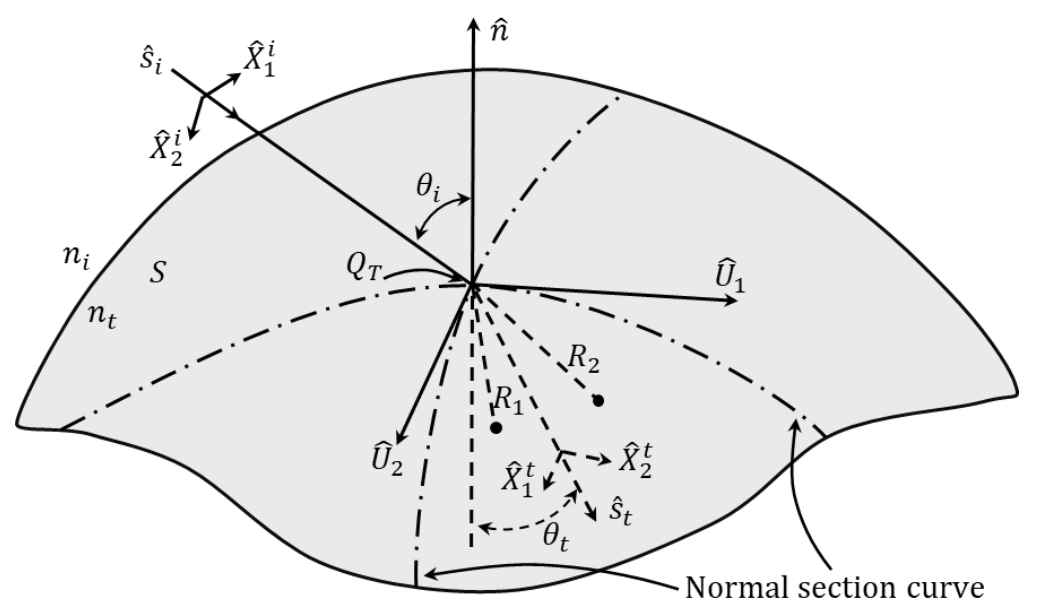

Figure E.3: Geometry for a transmission problem with the description of the curved surface $S$, incident ray and transmitted ray.

In the asymptotic procedure, when deriving the determinant of the Hessian matrix, i.e. $\operatorname{det}\left\{H_{\Phi}\left(Q_{T}\right)\right\}$, one can define two terms to make its expression compact, namely $\rho_{s}^{t}=\frac{1}{\rho_{1}^{t}}+\frac{1}{\rho_{2}^{t}}$ and $\rho_{m}^{t}=\frac{1}{\rho_{1}^{t} \rho_{2}^{t}}$. The final expressions of $\rho_{s}^{t}$ and $\rho_{m}^{t}$ are shown as below:

$$
\begin{aligned}
& \rho_{s}^{t}=\frac{1}{\left(n_{t} \cos \theta_{t}\right)^{2}}[ \\
& \frac{1}{\rho_{1}^{i}}\left\{\frac{n_{i}}{n_{t}}\left(n_{t}^{2}\left[1-\left(\hat{X}_{1}^{i} \cdot \hat{n}\right)^{2}\right]-n_{i}^{2}\left[\left(\hat{s}_{i} \cdot \widehat{U}_{2}\right)\left(\hat{X}_{1}^{i} \cdot \widehat{U}_{1}\right)+\left(\hat{s}_{i} \cdot \widehat{U}_{1}\right)\left(\hat{X}_{1}^{i} \cdot \widehat{U}_{2}\right)\right]^{2}\right)\right\}+ \\
& \frac{1}{\rho_{2}^{i}}\left\{\frac{n_{i}}{n_{t}}\left(n_{t}^{2}\left[1-\left(\hat{X}_{2}^{i} \cdot \hat{n}\right)^{2}\right]-n_{i}^{2}\left[\left(\hat{s}_{i} \cdot \widehat{U}_{2}\right)\left(\hat{X}_{2}^{i} \cdot \widehat{U}_{1}\right)+\left(\hat{s}_{i} \cdot \widehat{U}_{1}\right)\left(\hat{X}_{2}^{i} \cdot \widehat{U}_{2}\right)\right]^{2}\right)\right\}- \\
& \frac{1}{R_{1}}\left\{\frac{1}{n_{t}}\left(\left[n_{i}\left(\hat{s}_{i} \cdot \hat{n}\right)+n_{t} \cos \theta_{t}\right]\left[\left(n_{t}\right)^{2}-\left(n_{i}\right)^{2}\left(\hat{s}_{i} \cdot \widehat{U}_{2}\right)^{2}\right]\right)\right\}- \\
& \quad \frac{1}{R_{2}}\left\{\frac{1}{n_{t}}\left(\left[n_{i}\left(\hat{s}_{i} \cdot \hat{n}\right)+n_{t} \cos \theta_{t}\right]\left[\left(n_{t}\right)^{2}-\left(n_{i}\right)^{2}\left(\hat{s}_{i} \cdot \widehat{U}_{1}\right)^{2}\right]\right)\right\}
\end{aligned}
$$




$$
\begin{aligned}
& \rho_{m}^{t}=\frac{1}{\left(n_{t} \cos \theta_{t}\right)^{2}}\left[\frac{\left(n_{i}\right)^{2}}{\rho_{1}^{i} \rho_{2}^{i}}\left(\hat{s}_{i} \cdot \hat{n}\right)^{2}-\right. \\
& \frac{n_{i}}{R_{1} \rho_{1}^{i}}\left(\left[n_{i}\left(\hat{s}_{i} \cdot \hat{n}\right)+n_{t} \cos \theta_{t}\right]\left(\hat{X}_{1}^{i} \cdot \widehat{U}_{2}\right)^{2}\right)- \\
& \frac{n_{i}}{R_{2} \rho_{1}^{i}}\left(\left[n_{i}\left(\hat{s}_{i} \cdot \hat{n}\right)+n_{t} \cos \theta_{t}\right]\left(\hat{X}_{1}^{i} \cdot \widehat{U}_{1}\right)^{2}\right)- \\
& \frac{n_{i}}{R_{1} \rho_{2}^{i}}\left(\left[n_{i}\left(\hat{s}_{i} \cdot \hat{n}\right)+n_{t} \cos \theta_{t}\right]\left(\hat{X}_{2}^{i} \cdot \widehat{U}_{2}\right)^{2}\right)- \\
& \left.\frac{n_{i}}{R_{2} \rho_{2}^{i}}\left(\left[n_{i}\left(\hat{s}_{i} \cdot \hat{n}\right)+n_{t} \cos \theta_{t}\right]\left(\hat{X}_{2}^{i} \cdot \widehat{U}_{1}\right)^{2}\right)+\frac{1}{R_{1} R_{2}}\left(n_{i}\left(\hat{s}_{i} \cdot \hat{n}\right)+n_{t} \cos \theta_{t}\right)^{2}\right]
\end{aligned}
$$

Furthermore, by using $\rho_{s}^{t}$ and $\rho_{m}^{t}$, one can obtain the principal radii of curvature of the transmitted wave front, i.e. $\rho_{1}^{t}$ and $\rho_{2}^{t}$, as:

$$
\left\{\begin{array}{l}
\rho_{1}^{t}=\frac{\rho_{s}^{t}-\sqrt{\left(\rho_{s}^{t}\right)^{2}-4 \rho_{m}^{t}}}{2 \rho_{m}^{t}} \\
\rho_{2}^{t}=\frac{2}{\rho_{s}^{t}-\sqrt{\left(\rho_{s}^{t}\right)^{2}-4 \rho_{m}^{t}}}
\end{array}\right.
$$

As for the principal directions of the transmitted wave front, i.e. $\hat{X}_{1}^{t}$ and $\hat{X}_{2}^{t}$, they are not studied in the thesis, but they are useful information when one would like to analyze cascade transmitting systems such as a free-standing hyperbolic lens.

\section{E.4 Principal directions and principal radii of curvature of a specific surface of revolution}

An arbitrary surface of revolution can be represented by [13]:

$$
z=g(u), u=\frac{x^{2}+y^{2}}{2}
$$

If we define a signed parameter $K$ as follows:

$$
K= \pm \sqrt{1+2 u\left[\frac{d g(u)}{d u}\right]^{2}}
$$

where the sign " \pm " depends on the concavity of the surface, the principal radii of curvature can be found as:

$$
\left\{\begin{array}{l}
\frac{1}{R_{1}}=\frac{1}{K} \frac{d g(u)}{d u} \\
\frac{1}{R_{2}}=\frac{1}{K^{3}}\left[\frac{d g(u)}{d u}+2 u \frac{d^{2} g(u)}{d u^{2}}\right]
\end{array}\right.
$$


And the principal directions of the surface, $\widehat{U}_{1}$ and $\widehat{U}_{2}$, can be determined as:

$$
\left\{\begin{array}{l}
\widehat{U}_{1}=\frac{y}{\sqrt{x^{2}+y^{2}}} \hat{x}-\frac{x}{\sqrt{x^{2}+y^{2}}} \hat{y} \\
\widehat{U}_{2}=\frac{x}{K \sqrt{x^{2}+y^{2}}} \hat{x}+\frac{y}{K \sqrt{x^{2}+y^{2}}} \hat{y}+\frac{\sqrt{x^{2}+y^{2}}}{R_{1}} \hat{z}
\end{array}\right.
$$

- Parabolic reflector

For a parabolic reflector (parabola of revolution), the surface is expressed as:

$$
z=f-\frac{x^{2}+y^{2}}{4 f}=f-\frac{u}{2 f}
$$

and the derivatives in Eq. (E.19) are calculated as:

$$
\left\{\begin{array}{l}
\frac{d z}{d u}=-\frac{1}{2 f} \\
\frac{d^{2} z}{d u^{2}}=0
\end{array}\right.
$$

$K$ is determined to be positive:

$$
K=+\sqrt{1+\frac{u}{2 f^{2}}}
$$

Therefore, the principal radii of curvature are found as:

$$
\left\{\begin{array}{l}
R_{1}=-2 f\left(1+\frac{u}{2 f^{2}}\right)^{\frac{1}{2}} \\
R_{2}=-2 f\left(1+\frac{u}{2 f^{2}}\right)^{\frac{3}{2}}
\end{array}\right.
$$

Meanwhile, the principal directions can be obtained by substituting $K$ and $R_{1}$ in Eq. (E.20).

\section{- Elliptical lens and mirror}

For elliptical lenses and mirrors (ellipse of revolution), their surfaces can be expressed as:

$$
\left(\frac{z \pm c}{a}\right)^{2}+\frac{x^{2}+y^{2}}{b^{2}}=1 \Rightarrow z=c \pm a \sqrt{1-\frac{2 u}{b^{2}}}
$$

The derivatives of $z$ are calculated as:

$$
\left\{\begin{array}{l}
\frac{d z}{d u}=-\frac{a}{b^{2}}\left(1-\frac{2 u}{b^{2}}\right)^{-\frac{1}{2}} \\
\frac{\mathrm{d}^{2} z}{d u^{2}}=-\frac{a}{b^{4}}\left(1-\frac{2 u}{b^{2}}\right)^{-\frac{3}{2}}
\end{array}\right.
$$


For a lens, $K$ is determined to be negative:

$$
K_{\text {lens }}=-\sqrt{1+\frac{a^{2}}{b^{2}} \frac{2 u}{b^{2}-2 u}}
$$

and the principal radii of curvature are found as:

$$
\left\{\begin{array}{l}
R_{1, \text { lens }}=\frac{1}{a}\left(2 u c^{2}+b^{4}\right)^{\frac{1}{2}} \\
R_{2, \text { lens }}=\frac{1}{a b^{4}}\left(2 u c^{2}+b^{4}\right)^{\frac{3}{2}}
\end{array}\right.
$$

While for a mirror, $K$ is positive:

$$
K_{\text {mirror }}=\sqrt{1+\frac{a^{2}}{b^{2}} \frac{2 u}{b^{2}-2 u}}
$$

Moreover, the principal radii of curvature are found as:

$$
\left\{\begin{array}{l}
R_{1, \text { mirror }}=-\frac{1}{a}\left(2 u c^{2}+b^{4}\right)^{\frac{1}{2}} \\
R_{2, \text { mirror }}=-\frac{1}{a b^{4}}\left(2 u c^{2}+b^{4}\right)^{\frac{3}{2}}
\end{array}\right.
$$

- Hemispherical lens

In the case of a hemispherical lens (hemisphere of revolution), the surface is expressed as:

$$
(z-L)^{2}+x^{2}+y^{2}=R_{s p h}^{2} \Rightarrow z=L+\sqrt{R_{s p h}^{2}-2 u}
$$

And the derivatives of $z$ are calculated as:

$$
\left\{\begin{array}{l}
\frac{d z}{d u}=-\left(R^{2}-2 u\right)^{-\frac{1}{2}} \\
\frac{\mathrm{d}^{2} z}{d u^{2}}=-\left(R^{2}-2 u\right)^{-\frac{3}{2}}
\end{array}\right.
$$

$K$ is determined to be negative:

$$
K=-\sqrt{1+\frac{2 u}{R_{s p h}^{2}-2 u}}
$$

Consequently, the principal radii of curvature are found as:

$$
R_{1}=R_{2}=R_{s p h}
$$


- Hyperbolic lens

For a hyperbolic lens (hyperbola of revolution), the surface is expressed as:

$$
\left(\frac{z-c}{a}\right)^{2}-\frac{x^{2}+y^{2}}{b^{2}}=1 \Rightarrow z=c+a \sqrt{1+\frac{2 u}{b^{2}}}
$$

And the derivatives of $z$ are calculated as:

$$
\left\{\begin{array}{l}
\frac{d z}{d u}=\frac{a}{b^{2}}\left(1+\frac{2 u}{b^{2}}\right)^{-\frac{1}{2}} \\
\frac{\mathrm{d}^{2} z}{d u^{2}}=-\frac{a}{b^{4}}\left(1+\frac{2 u}{b^{2}}\right)^{-\frac{3}{2}}
\end{array}\right.
$$

$K$ is determined to be negative:

$$
K=-\sqrt{1+2 u\left[\frac{d g(u)}{d u}\right]^{2}}
$$

Therefore, the principal radii of curvature are found as:

$$
\left\{\begin{array}{l}
R_{1}=-\frac{1}{a}\left(2 u c^{2}+b^{4}\right)^{\frac{1}{2}} \\
R_{2}=-\frac{1}{a b^{4}}\left(2 u c^{2}+b^{4}\right)^{\frac{3}{2}}
\end{array}\right.
$$




\section{Appendix F Power budget}

In order to obtain the spreading factor, one can consider a power budget equation for each tube of rays. In other words, taking the law of conservation of energy in mind, the power incident on a medium should be equal to the power reflected plus the power transmitted from the interface. Fig. F.1 describes a case that an incident ray impinges on a point $Q$ at surface $S$. Part of the incident field is reflected back to the medium $\mathrm{I}\left(\varepsilon_{r 1}\right)$, while part of the field is transmitted into the medium II $\left(\varepsilon_{r 2}\right)$.

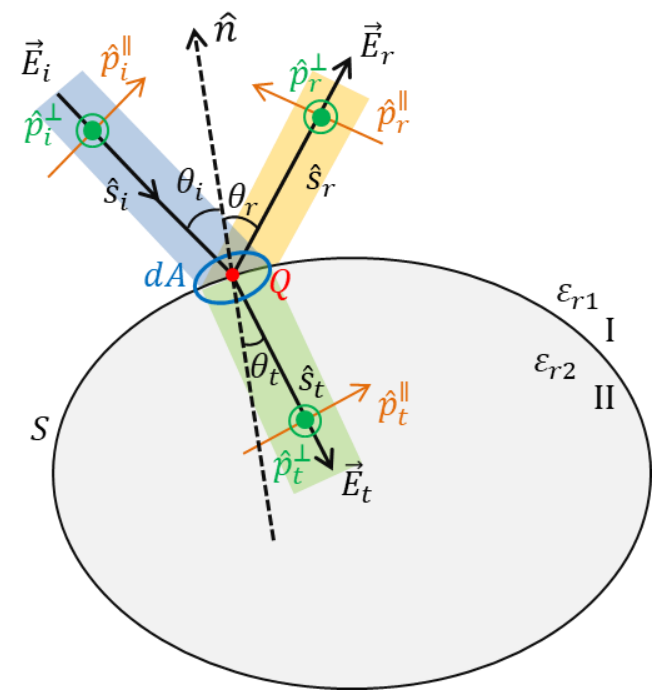

Figure F.1: Geometry for the description of power budget.

Firstly, we decompose the incident, reflected, and transmitted electric fields at $Q$ into TE and TM components:

$$
\vec{E}_{i, r, t}(Q)=E_{i, r, t}^{\perp}(Q) \hat{p}_{i, r, t}^{\perp}(Q)+E_{i, r, t}^{\|}(Q) \hat{p}_{i, r, t}^{\|}(Q)
$$

At each point $Q$, if we model each incident ray as a local plane wave, we can estimate the incident power crossing an infinitesimal area $d A$ centered at $Q$ :

$$
P_{i}^{\perp / \|}(Q)=\frac{\left|E_{i}^{\perp / \|}(Q)\right|^{2}}{2 \zeta_{1}} d A \cos \theta_{i}
$$

Same steps can also be performed for the reflected ray:

$$
P_{r}^{\perp / \|}(Q)=\frac{\left|E_{r}^{\perp / \|}(Q)\right|^{2}}{2 \zeta_{1}} d A \cos \theta_{r}=\frac{\left|\Gamma^{\perp / \|}(Q) E_{i}^{\perp / \|}(Q)\right|^{2}}{2 \zeta_{1}} d A \cos \theta_{i}
$$

and for the transmitted ray:

$$
P_{t}^{\perp / \|}(Q)=\frac{\left|E_{t}^{\perp / \|}(Q)\right|^{2}}{2 \zeta_{2}} d A \cos \theta_{t}=\frac{\left|\tau^{\perp / \|}(Q) E_{i}^{\perp / \|}(Q)\right|^{2}}{2 \zeta_{2}} d A \cos \theta_{t}
$$


If we substitute Eq. (F.2) in Eq. (F.3), we can represent the reflected power by the incident power:

$$
P_{r}^{\perp / \|}=P_{i}^{\perp / \|}\left|\Gamma^{\perp / \|}\right|^{2}
$$

Moreover, if we substitute Eq. (F.2) in Eq. (F.4), we can relate the transmitted power to the incident power:

$$
P_{t}^{\perp / \|}=P_{i}^{\perp / \|}\left|\tau^{\perp / \|}\right|^{2} \frac{\zeta_{1}}{\zeta_{2}} \frac{\cos \theta_{t}}{\cos \theta_{i}}
$$

Furthermore, when we consider the relation between the incident power and the reflected power, we can derive the following expression:

$$
P_{i}^{\perp / \|}-P_{r}^{\perp / \|}=\frac{\left|E_{i}^{\perp / \|}\right|^{2}\left(1-\left|\Gamma^{\perp / \|}\right|^{2}\right)}{2 \zeta_{1}} d A \cos \theta_{i}=\frac{\left|\tau^{\perp / \|} E_{i}^{\perp / \|}\right|^{2}}{2 \zeta_{2}} d A \cos \theta_{t}=P_{t}^{\perp / \|}
$$

It can be seen that Eq. (F.7) agrees with the law of conservation of energy. In the case of an interface between a PEC and air, there is no transmitted wave into the PEC, i.e. $\tau^{\perp / \|}=0$, the reflected power is equal to the incident power:

$$
P_{r}^{\perp / \|}=P_{i}^{\perp / \|}
$$




\section{Appendix G Spreading factor}

Spreading factors for all QO components are derived extensively in this appendix from section G.1 to G.5; and in section G.6 we describe some auxiliary derivations used when deriving spreading factors.

\section{G.1 Parabolic reflector}

In Fig. G.1, the incident ray is reflected at $Q_{R}$ and propagates to $Q_{F O} \cdot d A_{a}=\rho d \rho d \phi$ is the infinitesimal aperture area centered at $Q_{a}, d A_{R}=r^{2} \sin \theta d \theta d \phi$ is the area centered at $Q_{R}$, and $d A_{F O}=R_{F O}^{2} \sin \theta d \theta d \phi$ is the area centered at $Q_{F O}$.

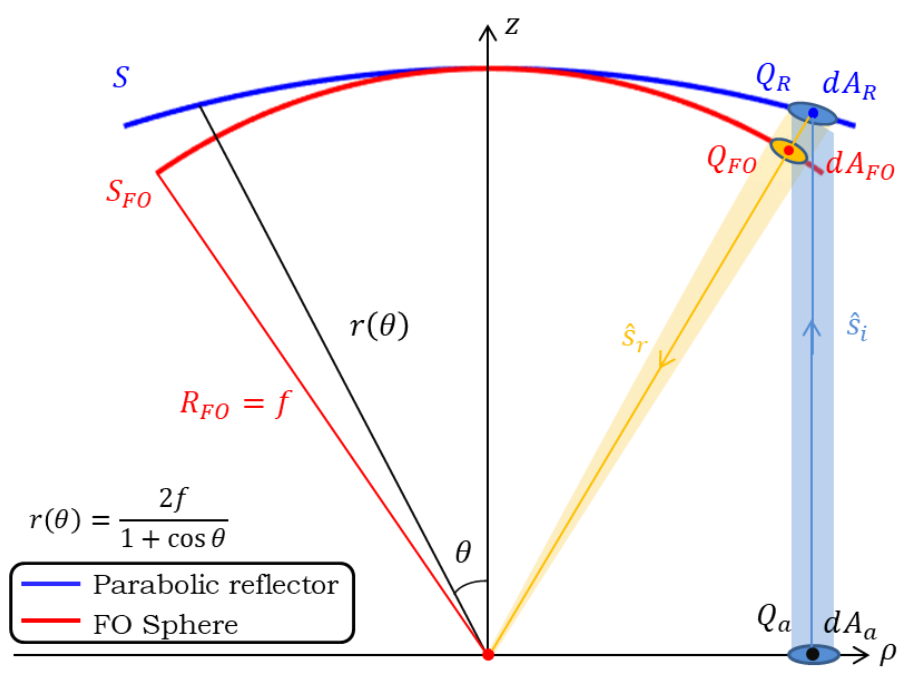

Figure G.1: A parabolic reflector as a reflecting surface.

The power within the incident ray tube should remain the same when propagating, also for the reflected ray tube:

$$
\left\{\begin{array}{l}
P_{i}^{\perp / \|}\left(Q_{a}\right)=P_{i}^{\perp / \|}\left(Q_{R}\right) \\
P_{G O}^{\perp / \|}\left(Q_{F O}\right)=P_{r}^{\perp / \|}\left(Q_{R}\right)
\end{array}\right.
$$

Considering the power budget introduced in Eq. (F.5), the incident power $P_{i}^{\perp / \|}$ and the reflected power $P_{r}^{\perp / \|}$ are related by the reflection coefficient $\Gamma^{\perp / \|}$ at $Q_{R}$. By substituting Eq. (G.1) in Eq. (F.5) one can obtain:

$$
P_{G O}^{\perp / \|}\left(Q_{F O}\right)=P_{i}^{\perp / \|}\left(Q_{a}\right)\left|\Gamma^{\perp / \|}\left(Q_{R}\right)\right|^{2}
$$

where $P_{i}^{\perp / \|}\left(Q_{a}\right)$ and $P_{G O}^{\perp / \|}\left(Q_{F O}\right)$ can be can be expressed as below: 


$$
\left\{\begin{array}{l}
P_{i}^{\perp / \|}\left(Q_{a}\right)=\frac{\left|E_{i}^{\perp / \|}\left(Q_{a}\right)\right|^{2}}{2 \zeta_{0}} d A_{a} \\
P_{G O}^{\perp / \|}\left(Q_{F O}\right)=\frac{\left|E_{G O}^{\perp / \|}\left(Q_{F O}\right)\right|^{2}}{2 \zeta_{0}} d A_{F O}
\end{array}\right.
$$

By substituting Eq. (G.3) in Eq. (G.2), we have the following relation:

$$
\frac{\left|E_{G O}^{\perp / \|}\left(Q_{F O}\right)\right|^{2}}{2 \zeta_{0}} d A_{F O}=\frac{\left|\Gamma^{\perp / \|}\left(Q_{R}\right) E_{i}^{\perp / \|}\left(Q_{a}\right)\right|^{2}}{2 \zeta_{0}} d A_{a}
$$

where $d A_{F O}=R_{F O}^{2} \sin \theta d \theta d \phi, d A_{a}=\rho d \rho d \phi$, and $\rho=r \sin \theta$. Eq. (G.4) can be simplified:

$$
\left|E_{G O}^{\perp / \|}\right|^{2}=\left|\Gamma^{\perp / \|} E_{i}^{\perp / \|}\right|^{2} \frac{r}{R_{F O}^{2}} \frac{d \rho}{d \theta}
$$

where $d \rho / d \theta$ can be calculated as follows:

$$
\frac{d \rho(\theta)}{d \theta}=\frac{d r(\theta)}{d \theta} \sin \theta+r(\theta) \cos \theta=\frac{2 f}{1+\cos \theta}=r(\theta)
$$

By substituting Eq. (G.6) in Eq. (G.5):

$$
\left|E_{G O}^{\perp / \|}\right|^{2}=\left|\Gamma^{\perp / \|} E_{i}^{\perp / \|}\right|^{2} \frac{r^{2}}{R_{F O}^{2}} \Rightarrow\left|E_{G O}^{\perp / \|}\right|=\left|\Gamma^{\perp / \|} E_{i}^{\perp / \|}\right| \frac{r}{R_{F O}}
$$

Consequently, the spreading factor can be expressed as below:

$$
S_{\text {pread }}(\theta)=\frac{\left|E_{G O}^{\perp / \|}\right|}{\left|\Gamma^{\perp / \|} E_{i}^{\perp / \|}\right|}=\frac{r(\theta)}{R_{F O}}=\frac{2}{1+\cos \theta}
$$




\section{G.2 Elliptical lens}

Fig. G.2 shows an elliptical lens as a transmitting surface. The incident ray is transmitted at $Q_{T}$ and propagates to $Q_{F O} \cdot d A_{a}=\rho d \rho d \phi$ is the aperture area centered at $Q_{a}, d A_{T}=$ $r^{2} \sin \theta d \theta d \phi$ is the area centered at $Q_{T}$, and $d A_{F O}=R_{F O}^{2} \sin \theta d \theta d \phi$ is the area centered at $Q_{F O}$.

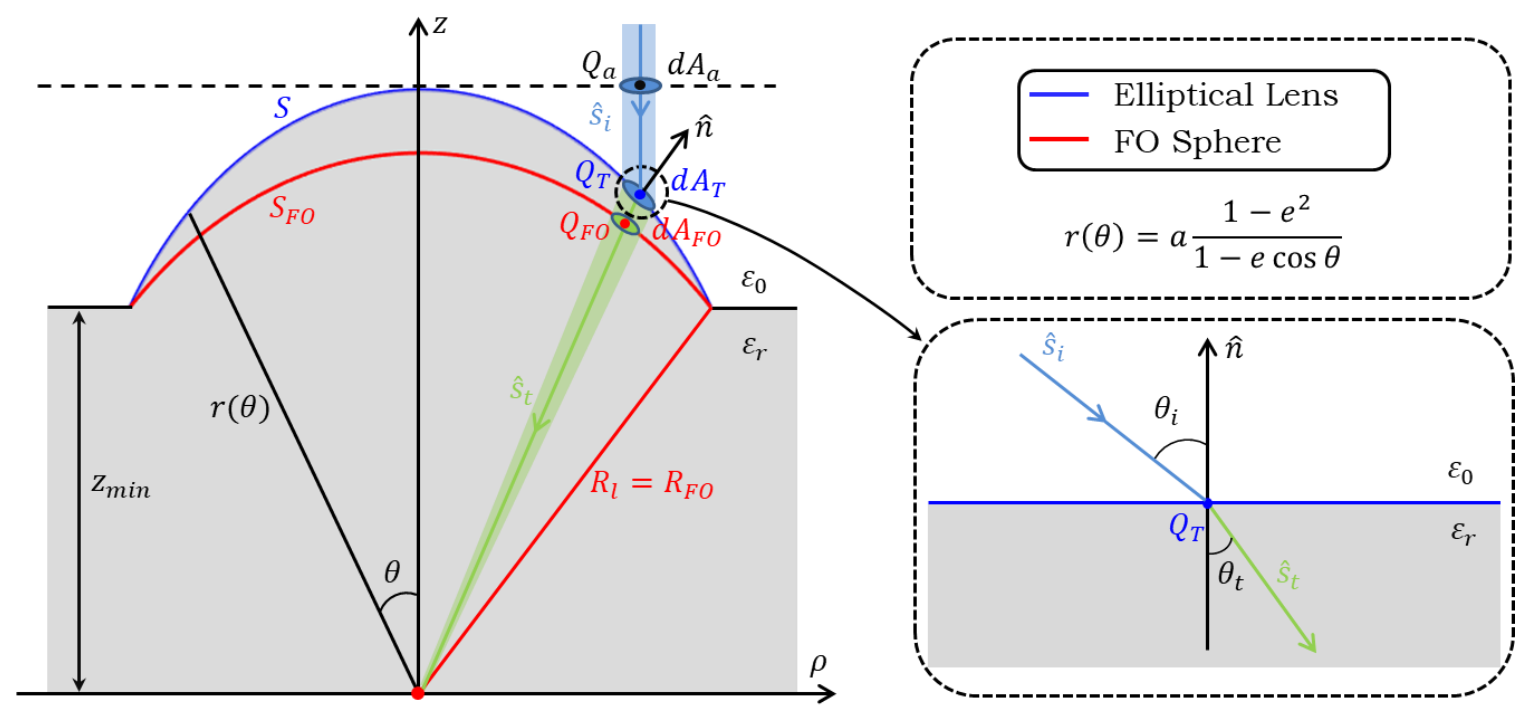

Figure G.2: An elliptical lens as a transmitting surface.

The power within the incident ray tube should remain the same when propagating, also for the transmitted ray tube:

$$
\left\{\begin{array}{l}
P_{i}^{\perp / \|}\left(Q_{a}\right)=P_{i}^{\perp / \|}\left(Q_{T}\right) \\
P_{G O}^{\perp / \|}\left(Q_{F O}\right)=P_{t}^{\perp / \|}\left(Q_{T}\right)
\end{array}\right.
$$

Considering the power budget introduced in Eq. (F.6), the incident power $P_{i}^{\perp / \|}$ and the transmitted power $P_{t}^{\perp / \|}$ are related by the transmission coefficient $\tau^{\perp / \|}$ at $Q_{T}$. By substituting Eq. (G.9) in Eq. (F.6) one can obtain:

$$
P_{G O}^{\perp / \|}\left(Q_{F O}\right)=P_{i}^{\perp / \|}\left(Q_{a}\right)\left|\tau^{\perp / \|}\left(Q_{T}\right)\right|^{2} \frac{\zeta_{0}}{\zeta_{d}} \frac{\cos \theta_{t}}{\cos \theta_{i}}
$$

where $P_{i}^{\perp / \|}\left(Q_{a}\right)$ is expressed in Eq. (G.3) and $P_{G O}^{\perp / \|}\left(Q_{F O}\right)$ is:

$$
P_{G O}^{\perp / \|}\left(Q_{F O}\right)=\frac{\left|E_{G O}^{\perp / \|}\left(Q_{F O}\right)\right|^{2}}{2 \zeta_{d}} d A_{F O}
$$

By substituting Eq. (G.3) and (G.11) in Eq. (G.10), one can obtain:

$$
\frac{\left|E_{G O}^{\perp / \|}\left(Q_{F O}\right)\right|^{2}}{2 \zeta_{d}} d A_{F O}=\frac{\left|E_{i}^{\perp / \|}\left(Q_{a}\right)\right|^{2}}{2 \zeta_{0}} d A_{a}\left|\tau^{\perp / \|}\left(Q_{T}\right)\right|^{2} \frac{\zeta_{0}}{\zeta_{d}} \frac{\cos \theta_{t}}{\cos \theta_{i}}
$$


where $d A_{F O}=R_{F O}^{2} \sin \theta d \theta d \phi, d A_{a}=\rho d \rho d \phi$, and $\rho=r \sin \theta$. Eq. (G.12) can be simplified:

$$
\left|E_{G O}^{\perp / \|}\right|^{2}=\left|\tau^{\perp / \|} E_{i}^{\perp / \|}\right|^{2} \frac{r}{R_{F O}^{2}} \frac{d \rho}{d \theta} \frac{\cos \theta_{t}}{\cos \theta_{i}}
$$

where $d \rho / d \theta$ can be calculated as follows:

$$
\frac{d \rho(\theta)}{d \theta}=\frac{d r(\theta)}{d \theta} \sin \theta+r(\theta) \cos \theta=r(\theta) \frac{\cos \theta-e}{1-e \cos \theta}
$$

and $\cos \theta_{t} / \cos \theta_{i}$ is derived in Appendix G.6 as:

$$
\frac{\cos \theta_{t}}{\cos \theta_{i}}=\frac{1-e \cos \theta}{\cos \theta-e}
$$

By substituting Eq. (G.14) and (G.15) in Eq. (G.13):

$$
\left|E_{G O}^{\perp / \|}\right|^{2}=\left|\tau^{\perp / \|} E_{i}^{\perp / \|}\right|^{2} \frac{r^{2}}{R_{F O}^{2}} \Rightarrow\left|E_{G O}^{\perp / \|}\right|=\left|\tau^{\perp / \|} E_{i}^{\perp / \|}\right| \frac{r}{R_{F O}}
$$

Therefore, the spreading factor is obtained as:

$$
S_{\text {pread }}(\theta)=\frac{\left|E_{G O}^{\perp / \|}\right|}{\left|\tau^{\perp / \|} E_{i}^{\perp / \|}\right|}=\frac{r(\theta)}{R_{F O}}=\frac{a\left(1-e^{2}\right)}{R_{l}(1-e \cos \theta)}
$$

\section{G.3 Hyperhemispherical lens}

In the case of a hyperhemispherical, as shown in Fig. G.3, the incident wave converges at the virtual point $O_{v}$, and the transmitted field converges at the center of the lens base, $O$. The incident field is transmitted at $Q_{T}$ and propagates to $Q_{F O} . d A_{T}=r_{v}^{2} \sin \theta_{v} d \theta_{v} d \phi_{v}$ is the area centered at $Q_{T}$, and $d A_{F O}=R_{F O}^{2} \sin \theta d \theta d \phi$ is the area centered at $Q_{F O}$.

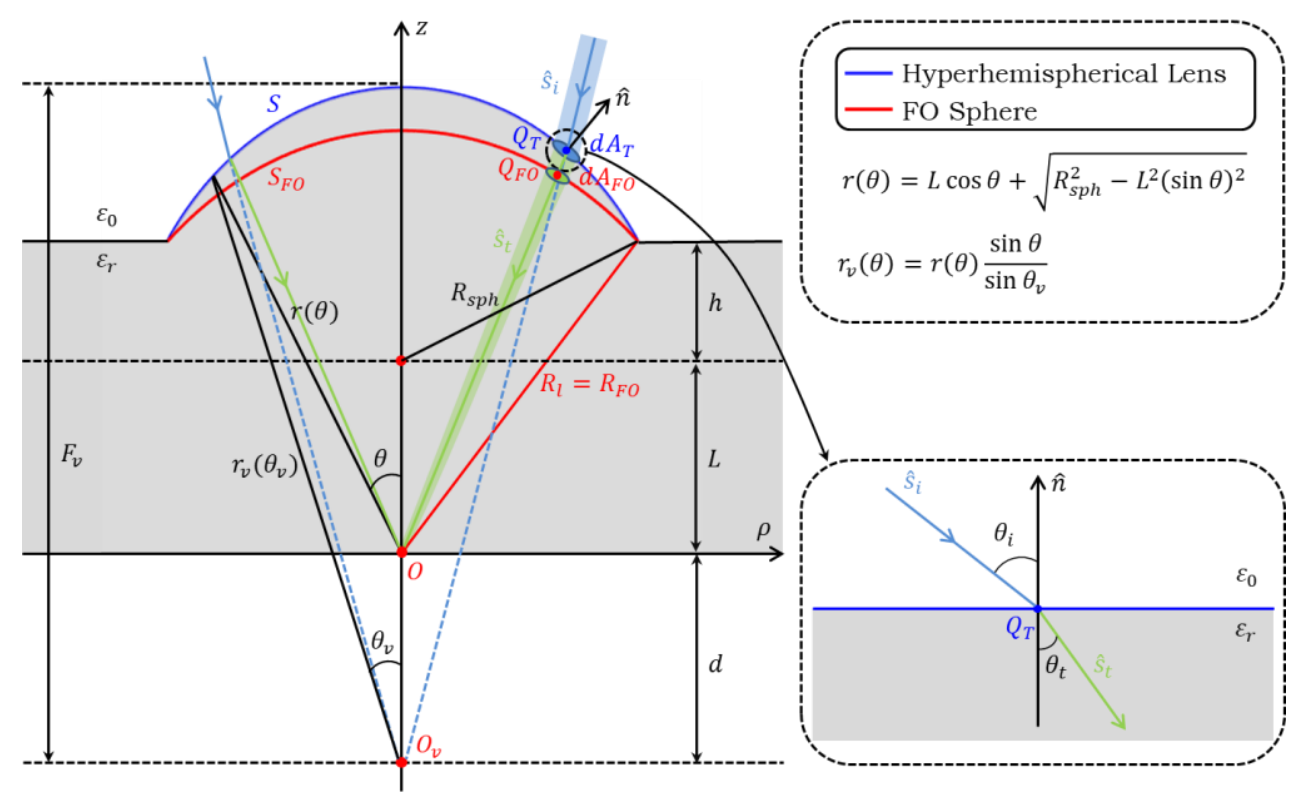

Figure G.3: A hyperhemispherical lens as a transmitting surface. 
Similar to an elliptical lens, considering the power budget we can obtain the following relation:

$$
\frac{\left|E_{G O}^{\perp / \|}\left(Q_{F O}\right)\right|^{2}}{2 \zeta_{d}} d A_{F O}=\frac{\left|E_{i}^{\perp / \|}\left(Q_{T}\right)\right|^{2}}{2 \zeta_{0}} d A_{T}\left|\tau^{\perp / \|}\left(Q_{T}\right)\right|^{2} \frac{\zeta_{0}}{\zeta_{d}} \frac{\cos \theta_{t}}{\cos \theta_{i}}
$$

where $d A_{F O}=R_{F O}^{2} \sin \theta d \theta d \phi, d A_{T}=r_{v}^{2} \sin \theta_{v} d \theta_{v} d \phi_{v}$ and $\phi_{v}=\phi$. Eq. (G.18) can be simplified:

$$
\left|E_{G O}^{\perp / \|}\right|^{2}=\left|\tau^{\perp / \|} E_{i}^{\perp / \|}\right|^{2} \frac{r_{v}^{2}}{R_{F O}^{2}} \frac{\sin \theta_{v}}{\sin \theta} \frac{d \theta_{v}}{d \theta} \frac{\cos \theta_{t}}{\cos \theta_{i}}
$$

It can be observed in Fig G.3 that the radial distance $r_{v}\left(\theta_{v}\right)$ is related to $r(\theta)$ :

$$
r_{v}\left(\theta_{v}\right)=r(\theta) \frac{\sin \theta}{\sin \theta_{v}}
$$

By substituting Eq. (G.20) in Eq. (G.19):

$$
\begin{gathered}
\left|E_{G O}^{\perp / \|}\right|^{2}=\left|\tau^{\perp / \|} E_{i}^{\perp / \|}\right|^{2} \frac{r^{2}}{R_{F O}^{2}} \frac{\sin \theta}{\sin \theta_{v}} \frac{d \theta_{v}}{d \theta} \frac{\cos \theta_{t}}{\cos \theta_{i}} \\
\Rightarrow\left|E_{G O}^{\perp / \|}\right|=\left|\tau^{\perp / \|} E_{i}^{\perp / \|}\right| \frac{r}{R_{F O}} \sqrt{\frac{\sin \theta}{\sin \theta_{v}} \frac{d \theta_{v}}{d \theta} \frac{\cos \theta_{t}}{\cos \theta_{i}}}
\end{gathered}
$$

Therefore, the spreading factor is expressed as:

$$
S_{\text {pread }}(\theta)=\frac{\left|E_{G O}^{\perp / \|}\right|}{\left|\tau^{\perp / \|} E_{i}^{\perp / \|}\right|}=\frac{r(\theta)}{R_{F O}} \sqrt{\frac{\sin \theta}{\sin \theta_{v}} \frac{d \theta_{v}}{d \theta}} \sqrt{\frac{\cos \theta_{t}}{\cos \theta_{i}}}
$$

Since $d \theta_{v} / d \theta, \cos \theta_{i}$, and $\cos \theta_{t}$ are too redundant to be expressed analytically, we calculate these terms numerically. 


\section{G.4 Hyperbolic lens}

Fig. G.4 shows a hyperbolic lens as a transmitting surface. The incident field is a plane wave propagating in the dielectric medium $\left(\varepsilon_{r}\right)$. It is transmitted at $Q_{T}$ and propagates to $Q_{F O} \cdot d A_{a}=$ $\rho d \rho d \phi$ is the aperture area centered at $Q_{a}, d A_{T}=r^{2} \sin \theta d \theta d \phi$ is the area centered at $Q_{T}$, and $d A_{F O}=R_{F O}^{2} \sin \theta d \theta d \phi$ is the area centered at $Q_{F O}$.

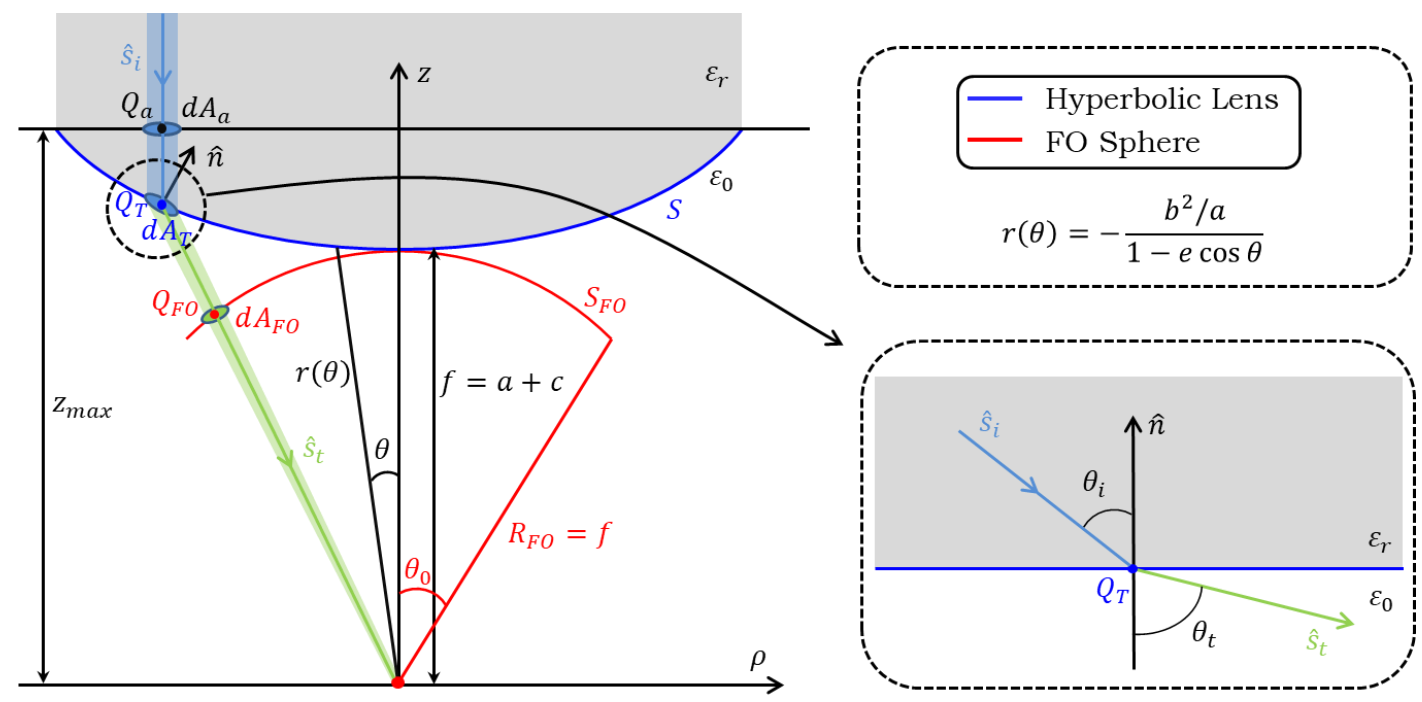

Figure G.4: A hyperbolic lens as a transmitting surface.

Similar to an elliptical lens, the power budget for a hyperbolic lens can be described as follows:

$$
\begin{aligned}
& P_{G O}^{\perp / \|}\left(Q_{F O}\right)=P_{i}^{\perp / \|}\left(Q_{a}\right)\left|\tau^{\perp / \|}\left(Q_{T}\right)\right|^{2} \frac{\zeta_{d}}{\zeta_{0}} \frac{\cos \theta_{t}}{\cos \theta_{i}} \\
\Rightarrow & \frac{\left|E_{G O}^{\perp / \|}\left(Q_{F O}\right)\right|^{2}}{2 \zeta_{0}} d A_{F O}=\frac{\left|E_{i}^{\perp / \|}\left(Q_{a}\right)\right|^{2}}{2 \zeta_{d}} d A_{a}\left|\tau^{\perp / \|}\left(Q_{T}\right)\right|^{2} \frac{\zeta_{d}}{\zeta_{0}} \frac{\cos \theta_{t}}{\cos \theta_{i}}
\end{aligned}
$$

where $d A_{F O}=R_{F O}^{2} \sin \theta d \theta d \phi, d A_{a}=\rho d \rho d \phi$, and $\rho=r \sin \theta$. Eq. (G.23) can be simplified:

$$
\left|E_{G O}^{\perp / \|}\right|^{2}=\left|\tau^{\perp / \|} E_{i}^{\perp / \|}\right|^{2} \frac{r}{R_{F O}^{2}} \frac{d \rho}{d \theta} \frac{\cos \theta_{t}}{\cos \theta_{i}}
$$

where $d \rho / d \theta$ and $\cos \theta_{t} / \cos \theta_{i}$ are described in Eq. (G.14) and (G.15), respectively. By substituting $d \rho / d \theta$ and $\cos \theta_{t} / \cos \theta_{i}$ in Eq. (G.24):

$$
\left|E_{G O}^{\perp / \|}\right|^{2}=\left|\tau^{\perp / \|} E_{i}^{\perp / \|}\right|^{2} \frac{r^{2}}{R_{F O}^{2}} \Rightarrow\left|E_{G O}^{\perp / \|}\right|=\left|\tau^{\perp / \|} E_{i}^{\perp / \|}\right| \frac{r}{R_{F O}}
$$

Therefore, the spreading factor is obtained as follows:

$$
S_{\text {pread }}(\theta)=\frac{\left|E_{G O}^{\perp / \|}\right|}{\left|\tau^{\perp / \|} E_{i}^{\perp / \|}\right|}=\frac{r(\theta)}{R_{F O}}=\frac{1-e}{1-e \cos \theta}
$$




\section{G.5 Elliptical mirror}

In the case of an elliptical mirror, incident field is emitted from a point source that can be placed at either the upper focus or the lower focus.

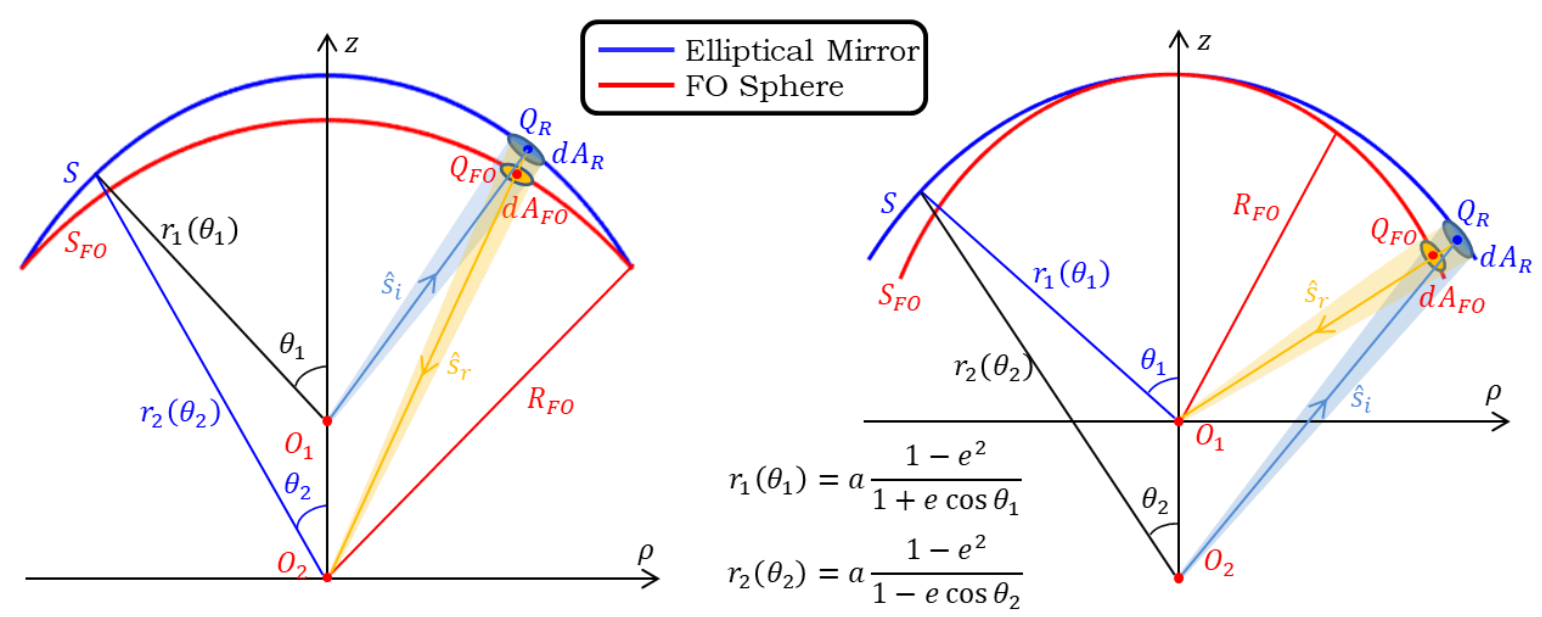

Figure G.5: An elliptical mirror as a reflecting surface. Left: source placed at the upper focus, $O_{1}$; Right: source placed at the lower focus, $\mathrm{O}_{2}$.

As it can be seen in Fig. G.5, the incident field is reflected at $Q_{R}$ and propagates to $Q_{F O}$. $d A_{R}$ is the area centered at $Q_{R}$, and $d A_{F O}$ is the area centered at $Q_{F O}$. Similar to a parabolic reflector, by considering the power budget between the incident power and the reflected power, one can derive the following relation:

$$
\frac{\left|E_{G O}^{\perp / \|}\left(Q_{F O}\right)\right|^{2}}{2 \zeta_{0}} d A_{F O}=\frac{\left|\Gamma^{\perp / \|}\left(Q_{R}\right) E_{i}^{\perp / \|}\left(Q_{R}\right)\right|^{2}}{2 \zeta_{0}} d A_{R}
$$

\section{- Case1: Source placed at the upper focus, $O_{1}$}

In this case, the area $d A_{R}$ is parameterized by $r_{1}\left(\theta_{1}\right): d A_{R}=r_{1} \sin \theta_{1} d \theta_{1} d \phi_{1}$, while $d A_{F O}$ is parameterized by $\left(\theta_{2}, \phi_{2}\right): d A_{F O}=R_{F O}^{2} \sin \theta_{2} d \theta_{2} d \phi_{2}$, where $\phi_{1}=\phi_{2}$. By substituting $d A_{F O}$ and $d A_{R}$ in Eq. (G.27):

$$
\begin{gathered}
\left|E_{G O}^{\perp / \|}\left(Q_{F O}\right)\right|^{2} R_{F O}^{2} \sin \theta_{2} d \theta_{2} d \phi_{2}=\left|\Gamma^{\perp / \|}\left(Q_{R}\right) E_{i}^{\perp / \|}\left(Q_{R}\right)\right|^{2} r_{1}^{2} \sin \theta_{1} d \theta_{1} d \phi_{1} \\
\Rightarrow \frac{\left|E_{G O}^{\perp / \|}\right|^{2}}{\left|\Gamma^{\perp / \|} E_{i}^{\perp / \|}\right|^{2}}=\frac{r_{1}^{2}}{R_{F O}^{2}} \frac{\sin \theta_{1}}{\sin \theta_{2}} \frac{d \theta_{1}}{d \theta_{2}}
\end{gathered}
$$

By using the relation between $\theta_{1}$ and $\theta_{2}$, one can calculate the following term:

$$
\frac{\sin \theta_{1}}{\sin \theta_{2}} \frac{d \theta_{1}}{d \theta_{2}}=\frac{r_{2}^{2}}{r_{1}} \frac{1}{2 c e+(1-A e) a\left(1-e^{2}\right)}
$$


and by substituting Eq. (G.29) in Eq. (G.28):

$$
\frac{\left|E_{G O}^{\perp / \|}\right|^{2}}{\left|\Gamma^{\perp / \|} E_{i}^{\perp / \|}\right|^{2}}=\frac{r_{1} r_{2}^{2}}{R_{F O}^{2}} \frac{1}{2 c e+(1-A e) a\left(1-e^{2}\right)}
$$

Consequently, the spreading factor can be expressed as:

$$
S_{\text {pread }}\left(\theta_{2}\right)=\frac{\left|E_{G O}^{\perp / \|}\right|}{\left|\Gamma^{\perp / \|} E_{i}^{\perp / \|}\right|}=\frac{\sqrt{r_{1}\left(\theta_{1}\right) r_{2}\left(\theta_{2}\right)}}{R_{F O}} \sqrt{\frac{a\left(1-e^{2}\right)}{2 c\left(e-\cos \theta_{2}\right)+a\left(1-e^{2}\right)}}
$$

- Case2: Source placed at the lower focus, $O_{2}$

In this case, the area $d A_{R}$ is parameterized by $r_{2}\left(\theta_{2}\right): d A_{R}=r_{2} \sin \theta_{2} d \theta_{2} d \phi_{2}$, while $d A_{F O}$ is parameterized by $\left(\theta_{1}, \phi_{1}\right): d A_{F O}=R_{F O}^{2} \sin \theta_{1} d \theta_{1} d \phi_{1}$. By substituting $d A_{F O}$ and $d A_{R}$ in Eq. (G.27):

$$
\begin{gathered}
\left|E_{G O}^{\perp / \|}\left(Q_{F O}\right)\right|^{2} R_{F O}^{2} \sin \theta_{1} d \theta_{1} d \phi_{1}=\left|\Gamma^{\perp / \|}\left(Q_{R}\right) E_{i}^{\perp / \|}\left(Q_{R}\right)\right|^{2} r_{2}^{2} \sin \theta_{2} d \theta_{2} d \phi_{2} \\
\Rightarrow \frac{\left|E_{G O}^{\perp / \|}\right|^{2}}{\left|\Gamma^{\perp / \|} E_{i}^{\perp / \|}\right|^{2}}=\frac{r_{2}^{2}}{R_{F O}^{2}} \frac{\sin \theta_{2}}{\sin \theta_{1}} \frac{d \theta_{2}}{d \theta_{1}}
\end{gathered}
$$

By using the relation between $\theta_{2}$ and $\theta_{1}$, one can calculate the following term:

$$
\frac{\sin \theta_{2}}{\sin \theta_{1}} \frac{d \theta_{2}}{d \theta_{1}}=\frac{r_{1}^{2}}{r_{2}} \frac{1}{2 c e+(1+A e) a\left(1-e^{2}\right)}
$$

By substituting Eq. (G.33) in Eq. (G.32):

$$
\frac{\left|E_{G O}^{\perp / \|}\right|^{2}}{\left|\Gamma^{\perp / \|} E_{i}^{\perp / \|}\right|^{2}}=\frac{r_{2} r_{1}^{2}}{R_{F O}^{2}} \frac{1}{2 c e+(1+A e) a\left(1-e^{2}\right)}
$$

Therefore, the spreading factor can be expressed as:

$$
S_{\text {pread }}\left(\theta_{1}\right)=\frac{\left|E_{G O}^{\perp / \|}\right|}{\left|\Gamma^{\perp / \|} E_{i}^{\perp / \|}\right|}=\frac{\sqrt{r_{1}\left(\theta_{1}\right) r_{2}\left(\theta_{2}\right)}}{R_{F O}} \sqrt{\frac{a\left(1-e^{2}\right)}{2 c\left(e+\cos \theta_{1}\right)+a\left(1-e^{2}\right)}}
$$




\section{G.6 Auxiliary derivations}

In this section, we mainly discuss some auxiliary derivations used when calculating spreading factors.

\section{- Parabolic reflector}

The incident propagation unit vector for a parabolic reflector is $\hat{s}_{i}=\hat{z}$, therefore, the incident and reflected angle can be calculated as follows:

$$
\cos \theta_{r}=\cos \theta_{i}=-\left(\hat{s}_{i} \cdot \hat{n}\right)=\sqrt{\frac{1+\cos \theta}{2}}
$$

\section{- Elliptical lens}

The incident and transmitted propagation unit vectors for an elliptical lens are $\hat{s}_{i}=-\hat{z}$ and $\hat{s}_{t}=-\hat{r}=-(\sin \theta \hat{\rho}+\cos \theta \hat{z})$, respectively, therefore, the incident and transmitted angle can be calculated as follows:

$$
\left\{\begin{array}{l}
\cos \theta_{i}=-\left(\hat{s}_{i} \cdot \hat{n}\right)=\frac{\cos \theta-e}{\sqrt{1+e^{2}-2 e \cos \theta}} \\
\cos \theta_{t}=-\left(\hat{s}_{t} \cdot \hat{n}\right)=\frac{1-e \cos \theta}{\sqrt{1+e^{2}-2 e \cos \theta}}
\end{array} \Rightarrow \frac{\cos \theta_{t}}{\cos \theta_{i}}=\frac{1-e \cos \theta}{\cos \theta-e}\right.
$$

- Hyperhemispherical lens

By observing Fig. G.3, one can derive the angle $\theta_{v}$ from $\theta$ :

$$
\theta_{v}=\tan ^{-1} \frac{r(\theta) \sin \theta}{r(\theta) \cos \theta+d}
$$

where $r(\theta)=L \cos \theta+\sqrt{R_{s p h}^{2}-L^{2}(\sin \theta)^{2}}, d=F_{v}-R_{s p h}-L$, and $F_{v}=R_{s p h}\left(\sqrt{\varepsilon_{r}}+1\right)$. The derivative of $\theta_{v}$ with respect to $\theta$ is:

$$
\frac{d \theta_{v}}{d \theta}=\frac{r^{2}+\frac{d r}{d \theta} \sin \theta d+r \cos \theta d}{(r \cos \theta+d)^{2}+(r \sin \theta)^{2}}
$$

where $d r / d \theta$ is expressed as follows:

$$
\frac{d r}{d \theta}=-L \sin \theta-L^{2} \sin \theta \cos \theta\left[R_{s p h}^{2}-L^{2} \sin ^{2} \theta\right]^{-0.5}
$$

Moreover, one can calculate the incident and transmitted angles numerically by using $\hat{s}_{i}=-\hat{r}_{v}$ (where $\left.\hat{r}_{v}=(d \vec{z}+\vec{r}) / r_{v}\right)$ and $\hat{s}_{t}=-\hat{r}$ :

$$
\left\{\begin{array}{l}
\cos \theta_{i}=-\left(\hat{s}_{i} \cdot \hat{n}\right) \\
\cos \theta_{t}=-\left(\hat{s}_{t} \cdot \hat{n}\right)
\end{array}\right.
$$




\section{Appendix H Auxiliary derivations for an elliptical mirror}

In section 4.2.2.2, we skip some steps when we simplify the linear phase and the compensation phase for an elliptical mirror. Therefore, in this appendix, we mainly discuss some simplifications used for an elliptical mirror with slightly off-broadside incidence.

\section{H.1 Simplification of terms in the linear phase}

When we calculate the linear phase term, there involve some simplifications for dot product.

- Case 1: Source placed at the upper focal plane

In this case the mirror is parameterized by $\vec{r}_{2}\left(\theta_{2}\right)$, and $\vec{r}_{1}\left(\theta_{1}\right)$ can be represented by using $\vec{r}_{2}\left(\theta_{2}\right)$ as:

$$
\begin{gathered}
\vec{r}_{1}=\vec{r}_{2}-2 c \hat{z} \Rightarrow \hat{r}_{1}=\frac{r_{2}}{r_{1}} \hat{r}_{2}-\frac{2 c}{r_{1}} \hat{z} \\
\Rightarrow \hat{r}_{1} \cdot \vec{\rho}_{s}=\left(\frac{r_{2}}{r_{1}} \hat{r}_{2}-\frac{2 c}{r_{1}} \hat{z}\right) \cdot \vec{\rho}_{s}=\frac{r_{2}}{r_{1}} \hat{r}_{2} \cdot \vec{\rho}_{s}
\end{gathered}
$$

If we define $r_{1}\left(\theta_{1}\right)$ and $r_{2}\left(\theta_{2}\right)$ by using $R_{1 l}$ and $R_{2 l}$, respectively,

$$
\left\{\begin{array}{l}
r_{1}\left(\theta_{1}\right)=a \frac{1-e^{2}}{1+e \cos \theta_{1}}=R_{1 l} \frac{1+e \cos \theta_{01}}{1+e \cos \theta_{1}} \\
r_{2}\left(\theta_{2}\right)=a \frac{1-e^{2}}{1-e \cos \theta_{2}}=R_{2 l} \frac{1-e \cos \theta_{02}}{1-e \cos \theta_{2}}
\end{array}\right.
$$

then the ratio $r_{2}\left(\theta_{2}\right) / r_{1}\left(\theta_{1}\right)$ can be expressed as follows:

$$
\frac{r_{2}\left(\theta_{2}\right)}{r_{1}\left(\theta_{1}\right)}=\frac{R_{2 l}}{R_{1 l}} \frac{1-e \cos \theta_{02}}{1+e \cos \theta_{01}} \frac{1+e \cos \theta_{1}}{1-e \cos \theta_{2}}=M_{1}\left(1+\Phi_{c o m a 1}\left(\theta_{2}\right)\right)
$$

where $M_{1}=R_{2 l} / R_{1 l}$ and $\Phi_{\text {coma } 1}\left(\theta_{2}\right)$ is:

$$
\begin{aligned}
& \Phi_{\text {coma } 1}\left(\theta_{2}\right)=- \\
& \frac{e^{2}\left(\cos \theta_{1} \cos \theta_{02}-\cos \theta_{2} \cos \theta_{01}\right)+e\left(\cos \theta_{01}-\cos \theta_{1}+\cos \theta_{02}-\cos \theta_{2}\right)}{\left(1-e \cos \theta_{2}\right)\left(1+e \cos \theta_{01}\right)}
\end{aligned}
$$

- Case 2: Source placed at the lower focal plane

In this case the mirror is parameterized by $\vec{r}_{1}\left(\theta_{1}\right)$, and $\vec{r}_{2}\left(\theta_{2}\right)$ can be represented by using $\vec{r}_{1}\left(\theta_{1}\right)$ as:

$$
\begin{gathered}
\vec{r}_{2}=\vec{r}_{1}+2 c \hat{z} \Rightarrow \hat{r}_{2}=\frac{r_{1}}{r_{2}} \hat{r}_{1}+\frac{2 c}{r_{2}} \hat{z} \\
\Rightarrow \hat{r}_{2} \cdot \vec{\rho}_{s}=\left(\frac{r_{1}}{r_{2}} \hat{r}_{1}+\frac{2 c}{r_{2}} \hat{z}\right) \cdot \vec{\rho}_{s}=\frac{r_{1}}{r_{2}} \hat{r}_{1} \cdot \vec{\rho}_{s}
\end{gathered}
$$


$r_{1}\left(\theta_{1}\right) / r_{2}\left(\theta_{2}\right)$ can be calculated by using Eq. (H.2):

$$
\frac{r_{1}\left(\theta_{1}\right)}{r_{2}\left(\theta_{2}\right)}=\frac{R_{1 l}}{R_{2 l}} \frac{1+e \cos \theta_{01}}{1-e \cos \theta_{02}} \frac{1-e \cos \theta_{2}}{1+e \cos \theta_{1}}=M_{2}\left(1+\Phi_{c o m a 2}\left(\theta_{1}\right)\right)
$$

where $M_{2}=R_{1 l} / R_{2 l}$, and $\Phi_{\text {coma2 }}\left(\theta_{1}\right)$ is:

$$
\begin{aligned}
& \Phi_{\text {coma } 2}\left(\theta_{1}\right)= \\
& \frac{e^{2}\left(\cos \theta_{1} \cos \theta_{02}-\cos \theta_{2} \cos \theta_{01}\right)+e\left(\cos \theta_{01}-\cos \theta_{1}+\cos \theta_{02}-\cos \theta_{2}\right)}{\left(1+e \cos \theta_{1}\right)\left(1-e \cos \theta_{02}\right)}
\end{aligned}
$$

\section{H.2 Simplification of compensation phase term}

The compensation phase term is complex and redundant to be implemented; therefore, here we will find a specific region where this phase can be simplified.

\section{- Case 1: Source placed at the upper focal plane}

In this case, the compensation phase term is expressed as follows:

$$
\Phi_{\text {comp } 1}\left(\theta_{2}\right)=k_{0}\left[\frac{\rho_{s}^{2}}{2 r_{1}}-\frac{\rho_{s}^{2}}{2 r_{1}}\left(\hat{r}_{1} \cdot \hat{\rho}_{s}\right)^{2}+\frac{\rho_{s}^{3}}{2 r_{1}^{2}}\left(\hat{r}_{1} \cdot \hat{\rho}_{s}\right)\right]
$$

Here we found $\frac{\rho_{s}^{2}}{2 r_{1}}$ varies slowly with respect to $\theta_{2}$, while $-\frac{\rho_{s}^{2}}{2 r_{1}}\left(\hat{r}_{1} \cdot \hat{\rho}_{s}\right)^{2}+\frac{\rho_{s}^{3}}{2 r_{1}^{2}}\left(\hat{r}_{1} \cdot \hat{\rho}_{s}\right)$ varies fast. Therefore, we can define a slow-varying term $\Phi_{\text {slow }}=k_{0} \frac{\rho_{s}^{2}}{2 r_{1}}$, and a fast-varying term $\Phi_{\text {fast }}=k_{0}\left[-\frac{\rho_{s}^{2}}{2 r_{1}}\left(\hat{r}_{1} \cdot \hat{\rho}_{s}\right)^{2}+\frac{\rho_{s}^{3}}{2 r_{1}^{2}}\left(\hat{r}_{1} \cdot \hat{\rho}_{s}\right)\right]$. Then Eq. (H.8) can be expressed as:

$$
\Phi_{\text {comp } 1}=\Phi_{\text {slow }}+\Phi_{\text {fast }}
$$

If we neglect $\Phi_{\text {fast }}$ for a specific phase error $\sigma_{p h, f}$, we have the following condition:

$$
-k_{0}\left(-\frac{\rho_{s}^{2}}{2 r_{1}}\left(\hat{r}_{1} \cdot \hat{\rho}_{s}\right)^{2}+\frac{\rho_{s}^{3}}{2 r_{1}^{2}}\left(\hat{r}_{1} \cdot \hat{\rho}_{s}\right)\right) \leq \sigma_{p h, f}
$$

The worst case in Eq. (H.10) happens when $\hat{r}_{1} \cdot \hat{\rho}_{s}=-\sin \theta_{01}$, which makes $r_{1}=R_{1 l}$. By considering the worst case, Eq. (H.10) can be simplified as:

$$
\frac{\sin \theta_{01}}{2 R_{1 l}^{2}} \rho_{s}^{3}+\frac{\left(\sin \theta_{01}\right)^{2}}{2 R_{1 l}} \rho_{s}^{2}-\frac{\sigma_{p h, f}}{k_{0}} \leq 0
$$

By solving the cubic equation, $a x^{3}+b x^{2}+c x+d=0$, with parameters: $a=\frac{\sin \theta_{01}}{2 R_{1 l}^{2}}, b=$ $\frac{\left(\sin \theta_{01}\right)^{2}}{2 R_{1 l}}, c=0, d=-\frac{\sigma_{p h, f}}{k_{0}}$, we can obtain the applicability region of Eq. (H.11). If we define the positive and real solution of the cubic equation as $x_{1}=\rho_{s, \text { max }}^{f}$, the region can be expressed as:

$$
\left|\rho_{s}\right| \leq \rho_{s, \max }^{f}
$$


Within this region, the fast-varying term $\Phi_{\text {fast }}$ can be neglected and the compensation phase can be simplified:

$$
\Phi_{\text {comp } 1} \approx \Phi_{\text {slow }}=k_{0} \frac{\rho_{s}^{2}}{2 r_{1}}
$$

Furthermore, we can represent the slow-varying term $\Phi_{\text {slow }}$ by a constant phase:

$$
\Phi_{\text {slow }} \approx k_{0} \frac{\rho_{s}^{2}}{2 R_{1 l}}
$$

and set the phase error as $\sigma_{p h, s}$ :

$$
\begin{gathered}
-k_{0} \frac{\rho_{s}^{2}}{2 R_{1 l}}-\left(-k_{0} \frac{\rho_{s}^{2}}{2 r_{1}}\right) \leq \sigma_{p h, s} \\
\Rightarrow \rho_{s}^{2}\left(\frac{1}{r_{1}}-\frac{1}{R_{1 l}}\right) \leq \frac{2 \sigma_{p h, s}}{k_{0}}
\end{gathered}
$$

The worst case happens when $\theta_{2}=0$, i.e. $r_{1}=a-c$, meaning Eq. (H.15) can be expressed as:

$$
\left|\rho_{s}\right| \leq \sqrt{\frac{2 \sigma_{p h, s}}{k_{0}}} \sqrt{\frac{R_{1 l}(a-c)}{R_{1 l}-(a-c)}}=\rho_{s, \max }^{s}
$$

In practice, when the f-number is large, we can always assume $\rho_{s, \max }^{f} \leq \rho_{s, \text { max }}^{S}$, meaning we can always approximate the compensation phase as a constant within $\left|\rho_{s}\right| \leq \rho_{s, \max }^{f}$ :

$$
\Phi_{\mathrm{comp} 1} \approx \frac{\rho_{s}^{2}}{2 R_{1 l}}
$$

\section{- Case 2: Source placed at the lower focal plane}

In this case, the compensation phase term is expressed as:

$$
\Phi_{\text {comp } 2}\left(\theta_{1}\right)=k_{0}\left[\frac{\rho_{s}^{2}}{2 r_{2}}-\frac{\rho_{s}^{2}}{2 r_{2}}\left(\hat{r}_{2} \cdot \hat{\rho}_{s}\right)^{2}+\frac{\rho_{s}^{3}}{2 r_{2}^{2}}\left(\hat{r}_{2} \cdot \hat{\rho}_{s}\right)\right]
$$

If we neglect the fast-varying term $\Phi_{\text {fast }}=k_{0}\left[-\frac{\rho_{s}^{2}}{2 r_{2}}\left(\hat{r}_{2} \cdot \hat{\rho}_{s}\right)^{2}+\frac{\rho_{s}^{3}}{2 r_{2}^{2}}\left(\hat{r}_{2} \cdot \hat{\rho}_{s}\right)\right]$ for a specific phase error $\sigma_{p h, f}$, we have the following condition:

$$
-k_{0}\left(-\frac{\rho_{s}^{2}}{2 r_{2}}\left(\hat{r}_{2} \cdot \hat{\rho}_{s}\right)^{2}+\frac{\rho_{s}^{3}}{2 r_{2}^{2}}\left(\hat{r}_{2} \cdot \hat{\rho}_{s}\right)\right) \leq \sigma_{p h, f}
$$

The worst case in Eq. (H.19) happens when $\hat{r}_{2} \cdot \hat{\rho}_{s}=-\sin \theta_{02}$, which makes $r_{2}=R_{2 l}$. By considering the worst case, Eq. (H.19) can be simplified as:

$$
\frac{\sin \theta_{02}}{2 R_{2 l}^{2}} \rho_{s}^{3}+\frac{\left(\sin \theta_{02}\right)^{2}}{2 R_{2 l}} \rho_{s}^{2}-\frac{\sigma_{p h}}{k_{0}} \leq 0
$$


By solving the cubic equation, $a x^{3}+b x^{2}+c x+d=0$, with parameters: $a=\frac{\sin \theta_{02}}{2 R_{2 l}^{2}}, b=$ $\frac{\left(\sin \theta_{02}\right)^{2}}{2 R_{2 l}}, c=0, d=-\frac{\sigma_{p h}}{k_{0}}$, we can obtain the applicability region of Eq. (H.20): $\left|\rho_{s}\right| \leq \rho_{s, \max }^{f}$, where $\rho_{s, \text { max }}^{f}$ is the positive and real solution of the cubic equation. Within this region, the fastvarying term $\Phi_{\text {fast }}$ can be neglected and the compensation phase can be simplified as:

$$
\Phi_{\text {comp } 2} \approx \Phi_{\text {slow }}=k_{0} \frac{\rho_{s}^{2}}{2 r_{2}}
$$

Furthermore, we can represent the slow-varying term $\Phi_{\text {slow }}$ by a constant phase:

$$
\Phi_{\text {slow }} \approx k_{0} \frac{\rho_{s}^{2}}{2 R_{2 l}}
$$

and set the phase error as $\sigma_{p h, s}$ :

$$
\begin{gathered}
-k_{0} \frac{\rho_{s}^{2}}{2 r_{2}}-\left(-k_{0} \frac{\rho_{s}^{2}}{2 R_{2 l}}\right) \leq \sigma_{p h, s} \\
\Rightarrow \rho_{s}^{2}\left(\frac{1}{R_{2 l}}-\frac{1}{r_{2}}\right) \leq \frac{2 \sigma_{p h, s}}{k_{0}}
\end{gathered}
$$

The worst case happens when $\theta_{1}=0$, i.e. $r_{2}=a+c$, meaning Eq. (H.23) can be expressed as:

$$
\left|\rho_{s r c}\right| \leq \sqrt{\frac{2 \sigma_{p h, s}}{k_{0}}} \sqrt{\frac{R_{2 l}(a+c)}{(a+c)-R_{2 l}}}=\rho_{s r c, \text { max }}^{s}
$$

In practice, when the f-number is large, we can always assume $\rho_{s, \max }^{f} \leq \rho_{s, \text { max }}^{s}$, meaning we can always approximate the compensation phase as a constant within $\left|\rho_{s}\right| \leq \rho_{s, \text { max }}^{f}$ :

$$
\Phi_{\text {comp } 2} \approx \frac{\rho_{S}^{2}}{2 R_{2 l}}
$$




\section{Appendix I Limit of the coma phase}

We define the limit of the coma phase as the maximum number of scanned beams where the focalized field is almost a linear translation of the broadside one, i.e., coma phase term can be neglected. The condition set for this limit is that the coma phase term should be less than $\sigma$ :

$$
\vec{k}_{\rho} \cdot \vec{\rho}_{f p} \delta_{n}(\theta) \leq \sigma
$$

\section{I.1 Parabolic reflector}

For a parabolic reflector, $\vec{k}_{\rho}=k_{0} \sin \theta \hat{\rho}, \vec{\rho}_{f p}=N \lambda_{0} f_{\#} \hat{\rho}_{f p}$. By substituting the expression of $\delta_{n}$ in Eq. (I.1):

$$
\begin{aligned}
& \frac{2 \pi}{\lambda_{0}} \sin \theta N \lambda_{0} f_{\#}\left(\hat{\rho} \cdot \hat{\rho}_{f p}\right) \frac{1-\cos \theta}{1+\cos \theta} \leq \sigma \\
& \Rightarrow N\left(\hat{\rho} \cdot \hat{\rho}_{f p}\right) \frac{\sin \theta(1-\cos \theta)}{1+\cos \theta} \leq \frac{\sigma}{2 \pi f_{\#}}
\end{aligned}
$$

If we define a function $f(\theta)$ as:

$$
f(\theta)=\frac{\sin \theta(1-\cos \theta)}{1+\cos \theta}
$$

We can find $f(\theta)$ is a monotonically increasing function within $\theta \in\left[0, \theta_{0}\right]$. Therefore, the worst case in Eq. (I.2) is when $\hat{\rho} \cdot \hat{\rho}_{f p}=1$ and $\theta=\theta_{0}$ :

$$
N \leq \frac{\sigma}{2 \pi f_{\#}} \frac{1+\cos \theta_{0}}{\sin \theta_{0}\left(1-\cos \theta_{0}\right)}
$$

Moreover, when $f_{\#}$ is large, $\sin \theta_{0}$ can be approximated by:

$$
\sin \theta_{0} \approx \frac{1}{2 f_{\#}} \Rightarrow \cos \theta_{0} \approx \frac{\sqrt{4 f_{\#}^{2}-1}}{2 f_{\#}}
$$

In this case, Eq. (I.4) is related to $f_{\#}$ as follows:

$$
N \leq \frac{\sigma}{\pi}\left[2 f_{\#}+\sqrt{4 f_{\#}^{2}-1}\right]^{2}
$$




\section{I.2 Elliptical lens}

For an elliptical lens, $\vec{k}_{\rho}=k_{d} \sin \theta \hat{\rho}, \vec{\rho}_{f p}=N \lambda_{d} f_{\#} \hat{\rho}_{f p}$. By substituting the expression of $\delta_{n}$ in Eq. (I.1):

$$
\begin{aligned}
& \frac{2 \pi}{\lambda_{d}} \sin \theta N \lambda_{d} f_{\#}\left(\hat{\rho} \cdot \hat{\rho}_{f p}\right) \frac{e\left(\cos \theta-\cos \theta_{0}\right)}{1-e \cos \theta} \leq \sigma \\
& \Rightarrow N\left(\hat{\rho} \cdot \hat{\rho}_{f p}\right) \frac{\sin \theta\left(\cos \theta-\cos \theta_{0}\right)}{1-e \cos \theta} \leq \frac{\sigma}{2 \pi f_{\#} e}
\end{aligned}
$$

If we define a function $f(\theta)$ as:

$$
f(\theta)=\frac{\sin \theta\left(\cos \theta-\cos \theta_{0}\right)}{1-e \cos \theta}
$$

we should find the value for $\theta \in\left[0, \theta_{0}\right]$ that maximizes $f(\theta)$. To do so we calculate the derivative of $f(\theta)$ with respect to $\theta$ :

$$
\begin{gathered}
\frac{\partial f(\theta)}{\partial \theta}=\frac{-e \sin ^{2} \theta}{(1-e \cos \theta)^{2}}\left[\cos \theta-\cos \theta_{0}\right]+\frac{\cos 2 \theta-\cos \theta \cos \theta_{0}}{1-e \cos \theta}=0 \\
\Rightarrow-e \cos ^{3} \theta+2 \cos ^{2} \theta-\cos \theta \cos \theta_{0}+e \cos \theta_{0}-1=0
\end{gathered}
$$

We should solve the cubic equation, $a x^{3}+b x^{2}+c x+d=0$, with parameters: $a=-e, b=$ 2, $c=-\cos \theta_{0}, d=e \cos \theta_{0}-1$, to find the angle $\theta_{\max }$ that maximize $f(\theta)$ :

$$
\theta_{\text {max }}=\cos ^{-1} x_{2}
$$

where $x_{2}=-\frac{1}{3 a}\left(b+C_{2}+\frac{\Delta_{0}}{C_{2}}\right), \Delta_{0}=b^{2}-3 a c, C_{2}=\left(-\frac{1}{2}+j \frac{1}{2} \sqrt{3}\right) \sqrt[3]{\frac{\Delta_{1}-\sqrt{\Delta_{1}^{2}-4 \Delta_{0}^{3}}}{2}}$, and $\Delta_{1}=2 b^{3}-9 a b c+27 a^{2} d$. For an elliptical lens, $\sin \theta_{0}$ can be calculated accurately as:

$$
\sin \theta_{0}=\frac{1}{2 f_{\#}} \Rightarrow \cos \theta_{0}=\frac{\sqrt{4 f_{\#}^{2}-1}}{2 f_{\#}}
$$

Therefore, the worst case in Eq. (I.7) happens when $\hat{\rho} \cdot \hat{\rho}_{f p}=1$ and $\theta=\theta_{\text {max }}$ :

$$
\begin{gathered}
N \leq \frac{\sigma}{2 \pi f_{\#} e} \frac{1-e \cos \theta_{\text {max }}}{\sin \theta_{\max }\left(\cos \theta_{\max }-\cos \theta_{0}\right)} \\
\Rightarrow N \leq \frac{\sigma}{\pi} \frac{1-e \cos \theta_{\max }}{e \sin \theta_{\max }} \frac{1}{2 f_{\#} \cos \theta_{\max }-\sqrt{4 f_{\#}^{2}-1}}
\end{gathered}
$$




\section{I.3 Hyperbolic lens}

In the case of a hyperbolic lens, $\vec{k}_{\rho}=k_{0} \sin \theta \hat{\rho}, \vec{\rho}_{f p}=N \lambda_{0} f_{\#} \hat{\rho}_{f p}$. By substituting the expression of $\delta_{n}$ in Eq. (I.1):

$$
\begin{aligned}
& \frac{2 \pi}{\lambda_{0}} \sin \theta N \lambda_{0} f_{\#}\left(\hat{\rho} \cdot \hat{\rho}_{f p}\right) \frac{e(\cos \theta-1)}{1-e \cos \theta} \leq \sigma \\
& \Rightarrow N\left(\hat{\rho} \cdot \hat{\rho}_{f p}\right) \frac{\sin \theta(\cos \theta-1)}{1-e \cos \theta} \leq \frac{\sigma}{2 \pi f_{\#} e}
\end{aligned}
$$

If we define a function $f(\theta)$ as:

$$
f(\theta)=\frac{\sin \theta(\cos \theta-1)}{1-e \cos \theta}
$$

We can find $f(\theta)$ is a monotonically increasing function within $\theta \in\left[0, \theta_{0}\right]$. Therefore, the worst case in Eq. (I.13) is when $\hat{\rho} \cdot \hat{\rho}_{f p}=1$ and $\theta=\theta_{0}$ :

$$
N \leq \frac{\sigma}{2 \pi f_{\#} e} \frac{1-e \cos \theta_{0}}{\sin \theta_{0}\left(\cos \theta_{0}-1\right)}
$$

Moreover, when $f_{\#}$ is large, $\sin \theta_{0}$ can be approximated by using Eq. (I.5). In such a case, Eq. (I.15) is related to $f_{\#}$ as:

$$
N \leq \frac{\sigma}{\pi e}\left[2 f_{\#}(e-1)\left(2 f_{\#}+\sqrt{4 f_{\#}^{2}-1}\right)-e\right]
$$

\section{I.4 Elliptical mirror}

For an elliptical mirror, we introduced the term $\Phi_{\text {coma }}$ instead of $\delta_{n}$; therefore, the condition should be modified:

$$
\vec{k}_{\rho} \cdot \vec{\rho}_{f p} \Phi_{c o m a} \leq \sigma
$$

\section{- Case1: Observation at lower focal plane}

In this case, $\vec{k}_{\rho}=k_{0} \sin \theta_{2} \hat{\rho}, \vec{\rho}_{f p}=N \lambda_{0} f_{\#} \hat{\rho}_{f p}$, and $\Phi_{\text {coma }}=\Phi_{\text {coma } 1}\left(\theta_{2}\right)$. Eq. (I.17) can be expressed as:

$$
\begin{aligned}
& \frac{2 \pi}{\lambda_{0}} \sin \theta_{2} N \lambda_{0} f_{\#}\left(\hat{\rho} \cdot \hat{\rho}_{f p}\right) \Phi_{c o m a 1}\left(\theta_{2}\right) \leq \sigma \\
& \Rightarrow N\left(\hat{\rho} \cdot \hat{\rho}_{f p}\right) \sin \theta_{2} \Phi_{c o m a 1}\left(\theta_{2}\right) \leq \frac{\sigma}{2 \pi f_{\#}}
\end{aligned}
$$

We can define a function $f\left(\theta_{2}\right)$ as:

$$
f\left(\theta_{2}\right)=\sin \theta_{2} \Phi_{c o m a 1}\left(\theta_{2}\right)
$$


The worst case in Eq. (I.18) happens when $\hat{\rho} \cdot \hat{\rho}_{f p}=1$ and $f\left(\theta_{2}\right)=f\left(\theta_{2}\right)_{\max }$. However, $f\left(\theta_{2}\right)$ is too complex that we cannot derive an analytical $\theta_{2}$ that maximizes $f\left(\theta_{2}\right)$. Therefore, we use two ways to calculate $N$. The first approach is that we calculate $f\left(\theta_{2}\right)_{\text {max }}$ numerically, and substitute it in Eq. (I.18):

$$
N \leq \frac{\sigma}{2 \pi f_{\#}} \frac{1}{f\left(\theta_{2}\right)_{\max }}
$$

The second approach is that we roughly assume $\theta_{2}=\frac{1}{2} \theta_{02}$ maximizes $f\left(\theta_{2}\right)$ :

$$
N \leq \frac{\sigma}{2 \pi f_{\#}} \frac{1}{\Phi_{\text {coma } 1}\left(\frac{1}{2} \theta_{02}\right) \sin \frac{1}{2} \theta_{02}}
$$

\section{- Case 2: Observation at upper focal plane}

In this case, $\vec{k}_{\rho}=k_{0} \sin \theta_{1} \hat{\rho}, \vec{\rho}_{f p}=N \lambda_{0} f_{\#} \hat{\rho}_{f p}$, and $\Phi_{\text {coma }}=\Phi_{\text {coma2 }}\left(\theta_{1}\right)$. Eq. (I.17) can be expressed as:

$$
\begin{aligned}
& \frac{2 \pi}{\lambda_{0}} \sin \theta_{1} N \lambda_{0} f_{\#}\left(\hat{\rho} \cdot \hat{\rho}_{f p}\right) \Phi_{c o m a 2}\left(\theta_{1}\right) \leq \sigma \\
& \Rightarrow N\left(\hat{\rho} \cdot \hat{\rho}_{f p}\right) \sin \theta_{1} \Phi_{c o m a 2}\left(\theta_{1}\right) \leq \frac{\sigma}{2 \pi f_{\#}}
\end{aligned}
$$

We can define a function $f\left(\theta_{1}\right)$ as:

$$
f\left(\theta_{1}\right)=\sin \theta_{1} \Phi_{\text {coma2 }}\left(\theta_{1}\right)
$$

The worst case in Eq. (I.22) happens when $\hat{\rho} \cdot \hat{\rho}_{f p}=1$ and $f\left(\theta_{1}\right)=f\left(\theta_{1}\right)_{\max }$. When we calculate $f\left(\theta_{1}\right)_{\max }$ numerically, and substitute it in Eq. (I.22):

$$
N \leq \frac{\sigma}{2 \pi f_{\#}} \frac{1}{f\left(\theta_{1}\right)_{\max }}
$$

When we roughly assume that $\theta_{1}=\frac{1}{2} \theta_{01}$ maximizes $f\left(\theta_{1}\right)$ :

$$
N \leq \frac{\sigma}{2 \pi f_{\#}} \frac{1}{\Phi_{\operatorname{coma} 2}\left(\frac{1}{2} \theta_{01}\right) \sin \frac{1}{2} \theta_{01}}
$$




\section{Appendix J Additional validation of the derived GO fields}

\section{J.1 Parabolic reflector}

- TM polarized plane wave

Fig. J.1 shows the X-component of the electric fields on the focal plane of a parabolic reflector with $D_{r}=100 \lambda_{0}\left(f_{0}=300 \mathrm{GHz}\right)$ and $f_{\#}^{r}=0.6$. The reflector is illuminated by a unitary TM polarized plane wave with the skew angle of $\theta_{s}=2.6\left(\lambda_{0} / D_{r}\right)=1.5^{\circ}, \phi_{s}=0^{\circ}$. And the focal plane fields evaluated by using the analytical FO and the GO-FO approaches are compared with the one obtained using the PO. Here the 3D PO field is plotted in the inset of the figure. As it can be seen in the figure, the agreement is within the accepted error margin inside the FO applicability region, for both the amplitude and the phase.

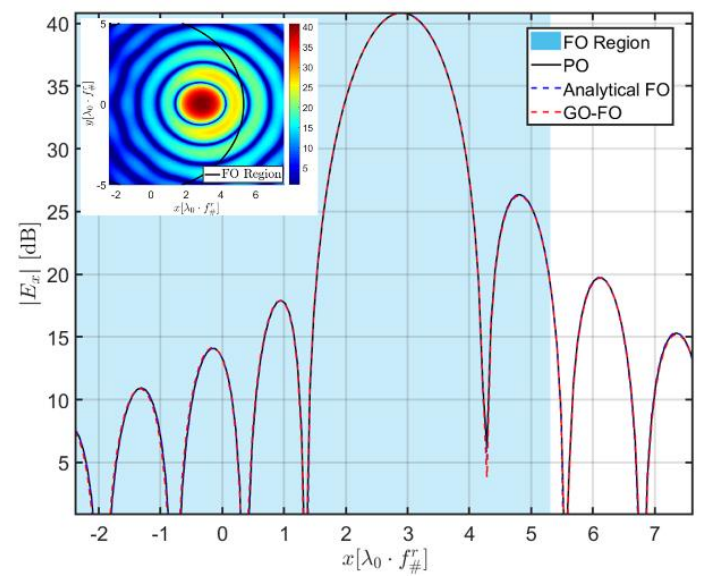

(a)

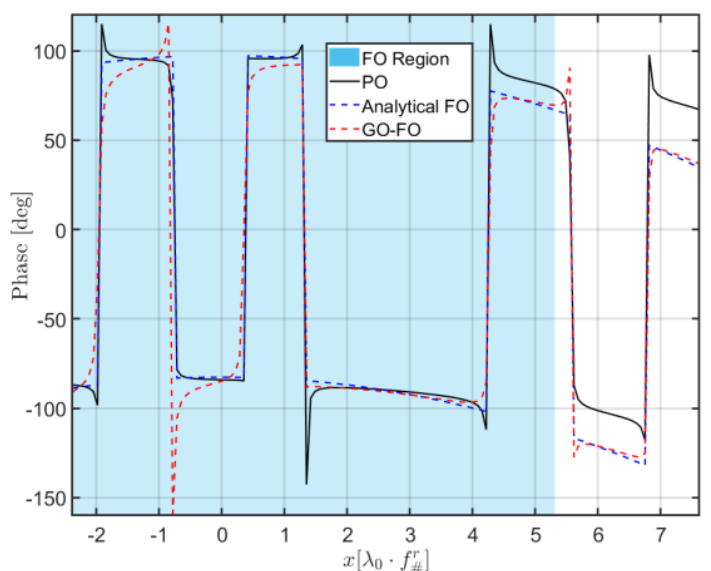

(b)

Figure J.1: The x-component of the electrics field on the focal plane of a parabolic reflector with $D_{r}=$ $100 \lambda_{0}\left(f_{0}=300 \mathrm{GHz}\right)$ and $f_{\#}^{r}=0.6$, illuminated by a unitary TM polarized plane wave with the skew angle of $\theta_{s}=2.6\left(\lambda_{0} / D_{r}\right)=1.5^{\circ}, \phi_{s}=0^{\circ}$. The focal plane fields calculated by using the analytical FO and the GO-FO approaches are compared with the one obtained using the PO: (a) Amplitude. (b) Phase. One of the main planes $(y=0)$ is shown. Blue region is the FO applicability region. Inset is the $3 \mathrm{D}$ PO field. 


\section{- TE polarized plane wave}

For the same reflector, when the plane wave is TE polarized with the skew angle of $\theta_{s}=$ $3.5\left(\lambda_{0} / D_{r}\right)=2^{\circ}, \phi_{s}=0^{\circ}$, the y-component of the electric field on the focal plane is shown in Fig. J.2. As it can be seen, the analytical FO and the GO-FO approaches are validated within the FO applicability region.

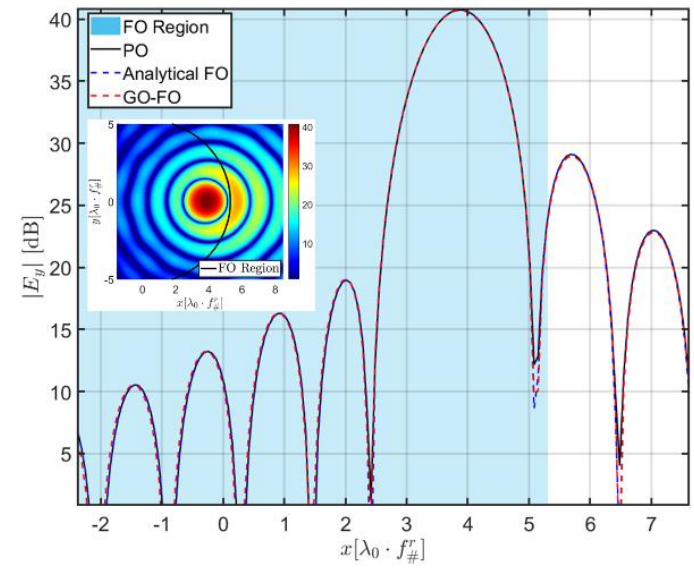

(a)

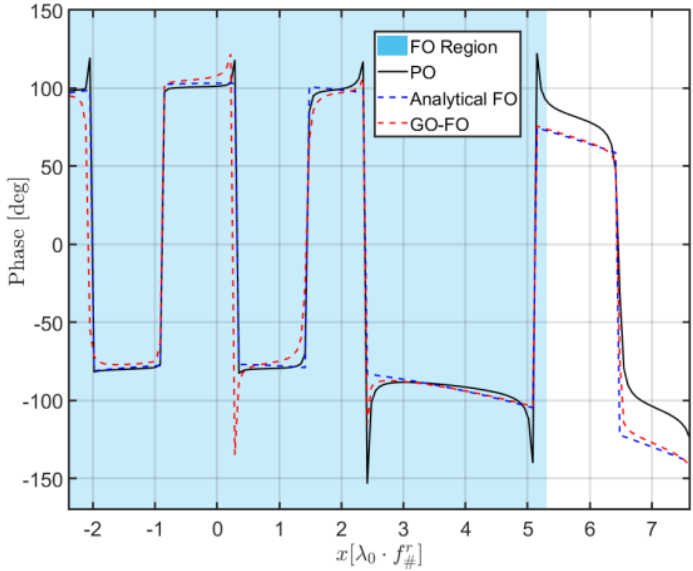

(b)

Figure J.2: The y-component of the electrics field on the focal plane of a parabolic reflector with $D_{r}=$ $100 \lambda_{0}\left(f_{0}=300 \mathrm{GHz}\right)$ and $f_{\#}^{r}=0.6$, illuminated by a unitary TE polarized plane wave with the skew angle of $\theta_{s}=3.5\left(\lambda_{0} / D_{r}\right)=2^{\circ}, \phi_{s}=0^{\circ}$. The focal plane fields calculated by using the analytical FO and the GO-FO approaches are compared with the one obtained using the PO: (a) Amplitude. (b) Phase. One of the main planes $(\boldsymbol{y}=\mathbf{0})$ is shown. Blue region is the FO applicability region. Inset is the 3D PO field.

\section{J.2 Elliptical lens}

An elliptical silicon $\left(\varepsilon_{r}=11.9\right)$ lens with $D_{l}=5 \lambda_{0}\left(f_{0}=300 \mathrm{GHz}\right)$ and $f_{\#}^{l}=0.6$ is introduced here. A quarter-wavelength matching layer at $300 \mathrm{GHz}$ made of Parylene $\left(\varepsilon_{m}=\right.$ 2.62 ) is applied. The lens is illuminated by a unitary TE polarized plane wave with the skew angle of $\theta_{s}=16^{\circ}, \phi_{s}=0^{\circ}$. Fig. J.3 shows the y-component of the electric fields on the focal plane of the lens. The focal plane field evaluated by using the GO-FO approach is compared with the one obtained using the PO. It can be seen in the figure that the agreement is within the accepted error margin inside the FO applicability region. 


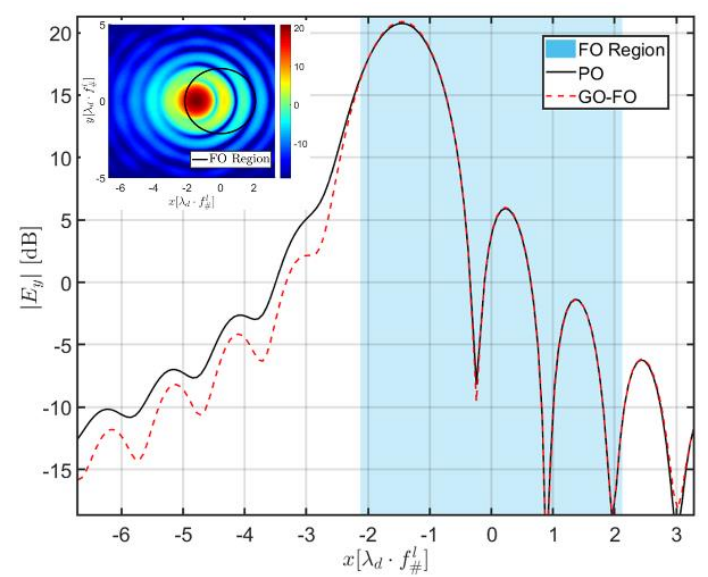

(a)

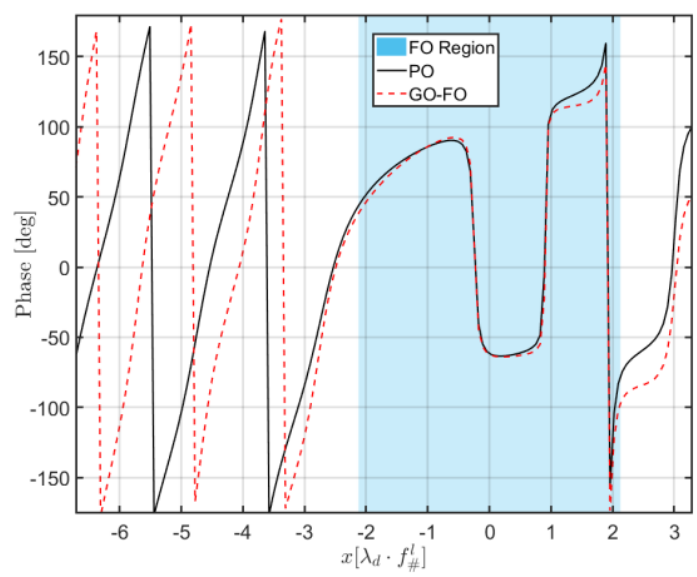

(b)

Figure J.3: The y-components of the electric fields on the focal plane of an elliptical silicon $\left(\varepsilon_{r}=11.9\right)$ lens with $D_{l}=5 \lambda_{0}\left(f_{0}=300 \mathrm{GHz}\right)$ and $f_{\#}^{l}=0.6$, illuminated by a unitary TE polarized plane wave with the skew angle of $\theta_{s}=16^{\circ}, \phi_{s}=0^{\circ}$. Here a quarter-wavelength matching layer at $300 \mathrm{GHz}$ made of Parylene $\left(\varepsilon_{m}=2.62\right)$ is applied. The focal plane field calculated by using the GO-FO approach is compared with the one obtained using the PO: (a) Amplitude. (b) Phase. One of the main planes $(\boldsymbol{y}=\mathbf{0})$ is shown. Blue region is the FO applicability region. Inset is the $3 \mathrm{D}$ PO field.

\section{J.3 Hemispherical lens}

\section{TM polarized plane wave}

Fig. J.4 shows the X-component of the electric fields on the focal plane of a hemispherical silicon $\left(\varepsilon_{r}=11.9\right)$ lens with $D_{h l}=5 \lambda_{0}\left(f_{0}=300 \mathrm{GHz}\right), f_{\#}^{h l}=0.6, R_{s p h}=2.6 \lambda_{0}$, and $L=$ $0.362 R_{s p h}$. A quarter-wavelength matching layer at $300 \mathrm{GHz}$ made of Parylene $\left(\varepsilon_{m}=2.62\right)$ is applied. The lens is illuminated by a unitary TM polarized plane wave with the skew angle of $\theta_{s}=15^{\circ}, \phi_{s}=0^{\circ}$. And the focal plane fields calculated by using the GO-FO is compared with the one obtained using the PO. It can be seen in the figure that the GO-FO approach is validated by the PO within the FO applicability region. 


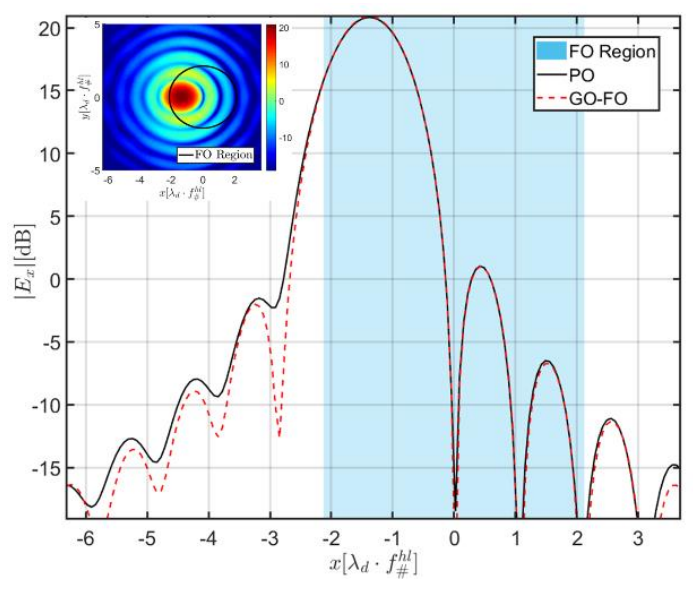

(a)

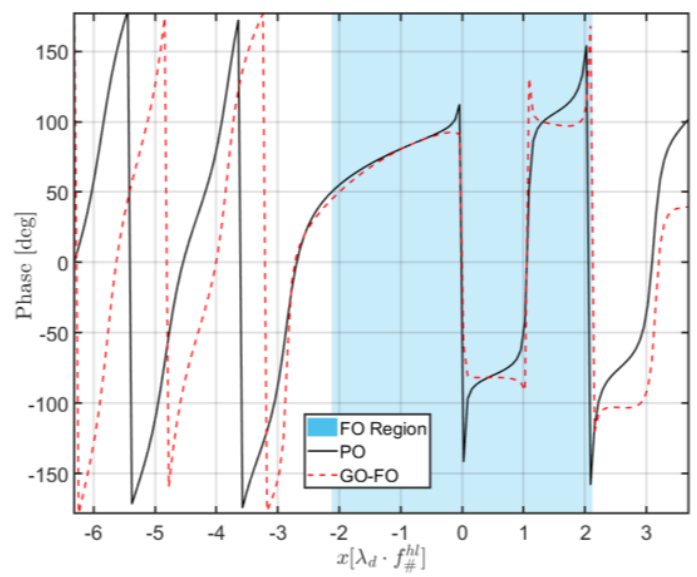

(b)

Figure J.4: The x-component of the electric fields on the focal plane of a hemispherical silicon $\left(\varepsilon_{r}=11.9\right)$ lens with $D_{h l}=5 \lambda_{0}\left(f_{0}=300 \mathrm{GHz}\right), f_{\#}^{h l}=0.6, R_{s p h}=2.6 \lambda_{0}$, and $L=0.362 R_{s p h}$, illuminated by a unitary TM polarized plane wave with the skew angle of $\theta_{s}=15^{\circ}, \phi_{s}=0^{\circ}$. Here a quarter-wavelength matching layer at 300 $\mathrm{GHz}$ made of Parylene $\left(\varepsilon_{m}=2.62\right)$ is applied. The focal field calculated by using the GO-FO approach is compared with the PO: (a) Amplitude. (b) Phase. One of the main planes $(y=0)$ is shown. Blue region is the FO applicability region. Inset is the $3 \mathrm{D}$ PO field.

\section{- TE polarized plane wave}

For the same hemispherical lens, when the plane wave is TE polarized with the skew angle of $\theta_{s}=20^{\circ}, \phi_{s}=0^{\circ}$, the y-component of the electric field on the focal plane is shown in Fig. J.5. As it can be seen, the GO-FO approach is validated within the FO applicability region.

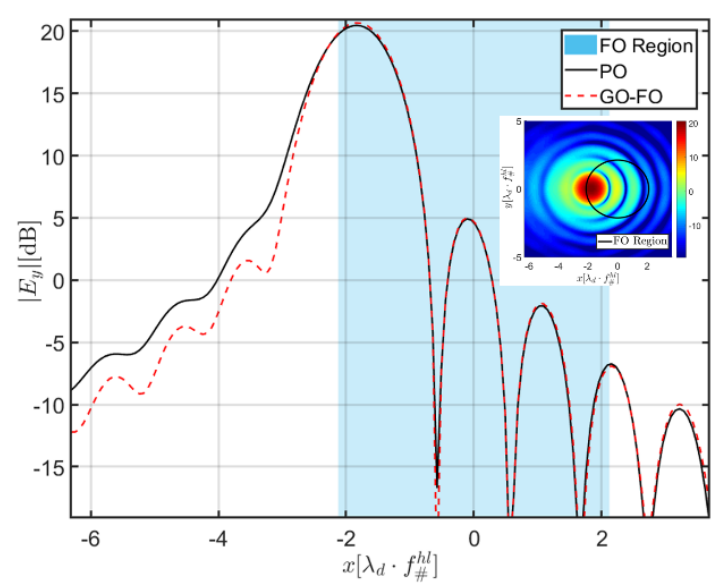

(a)

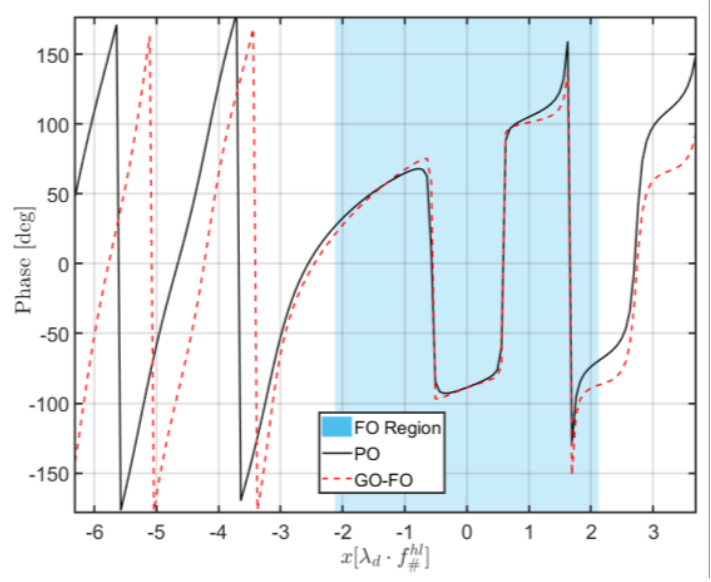

(b)

Figure J.5: The $\mathbf{y}$-component of the electric fields on the focal plane of a hemispherical silicon $\left(\varepsilon_{r}=11.9\right)$ lens with $D_{h l}=5 \lambda_{0}\left(f_{0}=300 \mathrm{GHz}\right), f_{\#}^{h l}=0.6, R_{s p h}=2.6 \lambda_{0}$, and $L=0.362 R_{s p h}$, illuminated by a unitary TE polarized plane wave with the skew angle of $\theta_{s}=20^{\circ}, \phi_{s}=0^{\circ}$. Here a quarter-wavelength matching layer at 300 GHz made of Parylene $\left(\varepsilon_{m}=2.62\right)$ is applied. The focal field calculated by using the GO-FO is compared with the PO: (a) Amplitude. (b) Phase. One of the main planes $(y=0)$ is shown. Blue region is the FO applicability region. Inset is the 3D PO field. 


\section{J.4 Hyperbolic lens}

Fig. J.6 shows the y-component of the electric fields on the focal plane of a hyperbolic plastic $\left(\varepsilon_{r}=2\right)$ lens with $D_{h}=100 \lambda_{0}\left(f_{0}=300 \mathrm{GHz}\right)$ and $f_{\#}^{h}=1$. The incident field is a unitary TE polarized plane wave with the skew angle of $\theta_{s}=3.7\left(\lambda_{d} / D_{h}\right)=1.5^{\circ}, \phi_{s}=0^{\circ}$. The focal plane fields evaluated by using the analytical FO and the GO-FO are compared with the one obtained using the PO. As it can be seen in the figure, for the amplitude comparison, the analytical FO and the GO-FO are validated by the PO within the FO applicability region. For the phase comparison, the analytical FO is still very good while the GO-FO shows some acceptable error. This is due to the fact that the interpolation operation involved in calculating the GO ray fields for a hyperbolic lens is not very accurate for a small f-number geometry.

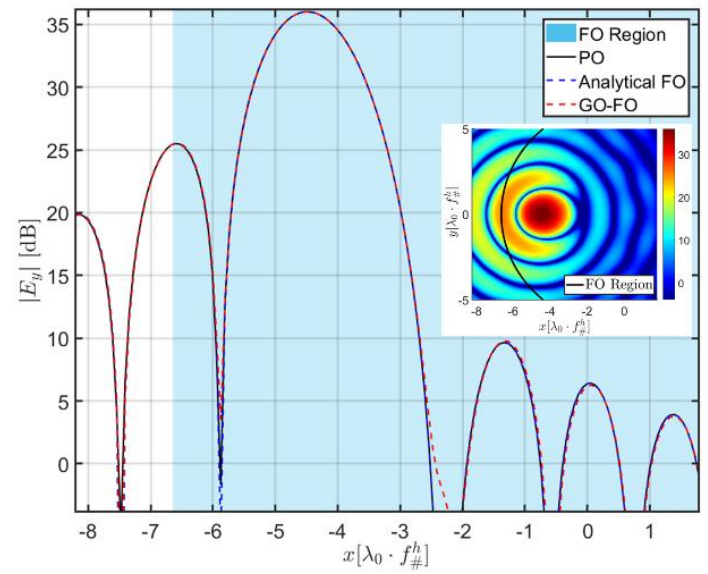

(a)

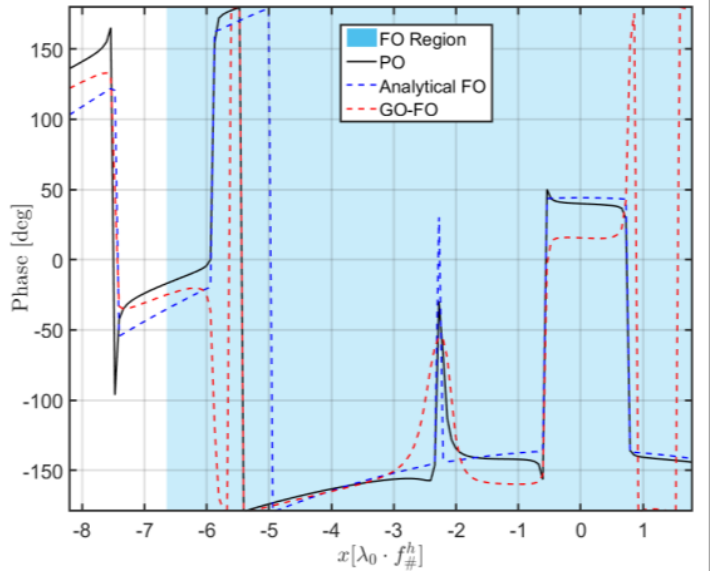

(b)

Figure J.6: The y-component of the electric fields on the focal plane of a hyperbolic plastic $\left(\varepsilon_{r}=2\right)$ lens with $D_{h}=100 \lambda_{0}\left(f_{0}=300 \mathrm{GHz}\right)$ and $f_{\#}^{h}=1$, illuminated by a unitary TE polarized plane wave with the skew angle of $\theta_{s}=3.7\left(\lambda_{d} / D_{h}\right)=1.5^{\circ}, \phi_{s}=0^{\circ}$. The focal plane fields calculated by using the analytical FO and the GO-FO are compared with the one obtained using the PO: (a) Amplitude. (b) Phase. One of the main planes $(y=0)$ is shown. Blue region is the FO applicability region. Inset is the 3D PO field. 


\section{J.5 Elliptical Mirror}

Here we consider a mirror with the diameter of $D_{m}=100 \lambda_{0}\left(f_{0}=300 \mathrm{GHz}\right)$, the semimajor axis of $a=80 \lambda_{0}$, and the focal distance of $c=20 \lambda_{0}$, i.e. the eccentricity is $e=0.25$. A unitary Huygens source is placed at the lower focal plane, with the electric current oriented along $\hat{y}$ and a displacement in $\mathrm{x}$-direction, $x_{s}=6.6 \lambda_{0} f_{\#}^{m}$. In this case, the f-number is calculated as $f_{\#}^{m}=0.6$. Fig. J.7 shows the y-component of the electric fields on the focal plane of the mirror. The focal plane fields evaluated by using the analytical FO and the GO-FO approaches are compared with the one obtained using the PO. As it can be seen in the figure, the agreement is within the accepted error margin inside the FO applicability region, for both the amplitude and the phase.

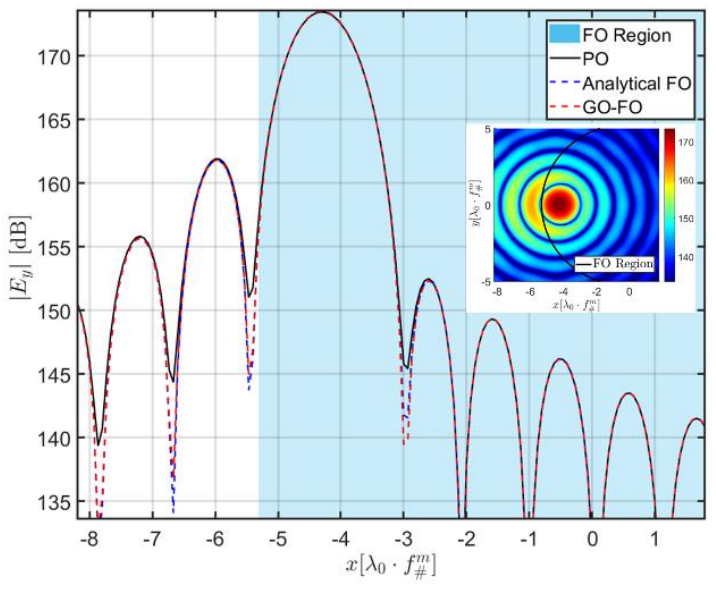

(a)

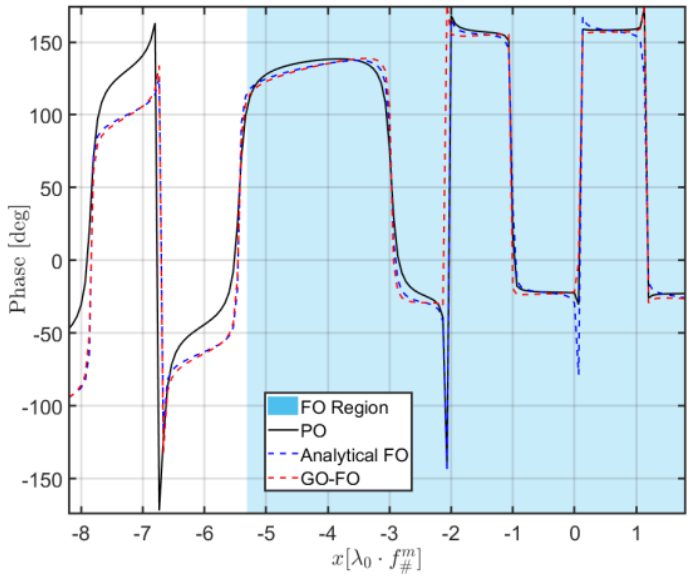

(b)

Figure J.7: The y-component of the electric fields on the focal plane of an elliptical mirror with $D_{m}=$ $100 \lambda_{0}\left(f_{0}=300 \mathrm{GHz}\right), f_{\#}^{m}=0.6$ and $e=0.25$. The mirror is illuminated by a unitary Huygens source placed at the lower focal plane, with the electric current oriented along $\hat{y}$ and a displacement in $\mathrm{x}$-direction, $x_{s}=$ $6.6 \lambda_{0} f_{\#}^{m}$. The focal plane fields evaluated by using the analytical FO and the GO-FO approaches are compared with the one obtained using PO: (a) Amplitude. (b) Phase. One of the main planes $(\boldsymbol{y}=\mathbf{0})$ is shown. Blue region is the FO applicability region. Inset is the $3 \mathrm{D}$ PO field. 


\section{Appendix K TE validation of the coherent FO}

\section{K.1 Parabolic reflector}

A parabolic reflector with the diameter of $D_{r}=500 \lambda_{0}\left(f_{0}=300 \mathrm{GHz}\right)$ and the f-number of $f_{\#}^{r}=4$ is introduced. The incident field is a unitary TE polarized plane wave with the skew angle of $\theta_{s}=24.4\left(\lambda_{0} / D_{r}\right)=2.8^{\circ}, \phi_{s}=0^{\circ}$, i.e. the flash point is $\vec{\rho}_{f p} \approx 24.4 \lambda_{0} f_{\#}^{r} \hat{x}$. The linearization point is chosen the as the flash point. Fig. K.1 shows the y-component of the electric fields on the focal plane of the hyperbolic lens. The focal plane field calculated by using the coherent FO is compared with the one obtained using the analytical FO. It can be seen that the coherent FO is validated inside the PWS applicability region, for both the amplitude and the phase.

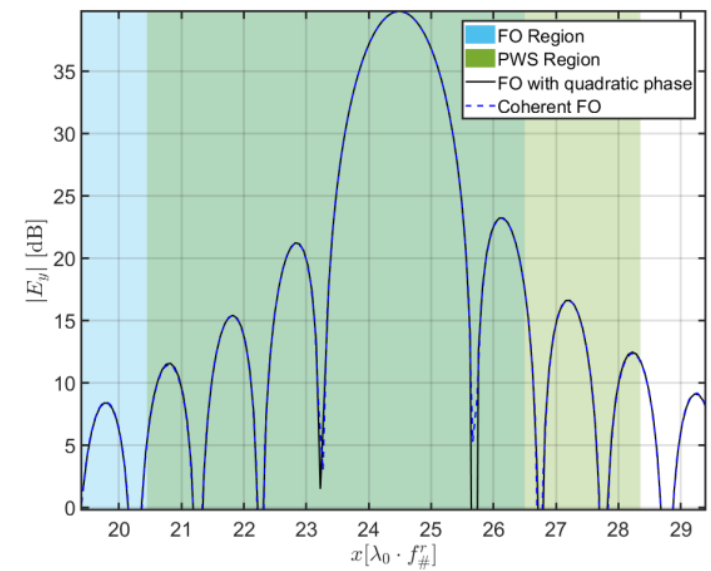

(a)

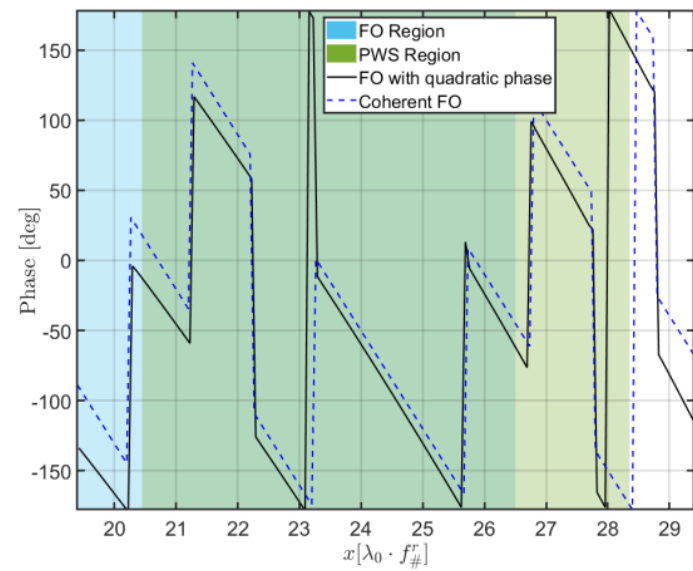

(b)

Figure K.1: The y-component of the electric fields on the focal plane of a parabolic reflector with $D_{r}=500 \lambda_{0}$ $\left(f_{0}=300 \mathrm{GHz}\right)$ and $f_{\#}^{r}=4$, illuminated by a unitary TE polarized plane wave with the skew angle of $\theta_{s}=$ $24.4\left(\lambda_{0} / D_{r}\right)=2.8^{\circ}, \phi_{s}=0^{\circ}$. The linearization point is chosen the same the flash point, i.e. $\vec{\rho}_{o} \approx 24.4 \lambda_{0} f_{\#}^{r} \hat{x}$. And the focal plane field calculated by using the coherent FO is compared with the one obtained using the analytical FO: (a) Amplitude. (b) Phase. One of the main planes $(y=0)$ is shown. Blue region is the FO applicability region and green region is the PWS applicability region. 


\section{K.2 Elliptical lens}

Fig. K.2 shows the y-component of the electric fields on the focal plane of an elliptical silicon $\left(\varepsilon_{r}=11.9\right)$ lens with $D_{l}=5 \lambda_{0}\left(f_{0}=300 \mathrm{GHz}\right)$ and $f_{\#}^{l}=0.6$. A quarter-wavelength matching layer at $300 \mathrm{GHz}$ made of Parylene $\left(\varepsilon_{m}=2.62\right)$ is applied. The lens is illuminated by a unitary TE polarized plane wave with the skew angle of $\theta_{s}=16^{\circ}, \phi_{s}=0^{\circ}$, i.e. $\vec{\rho}_{f p} \approx$ $-1.4 \lambda_{d} f_{\#}^{l} \hat{x}$. The linearization point is chosen the as the flash point. And the focal plane field evaluated by using the coherent FO is compared with the one obtained using the GO-FO. As it can be seen, the coherent FO is in excellent agreement with the GO-FO, for both the amplitude and the phase, within the PWS applicability region.

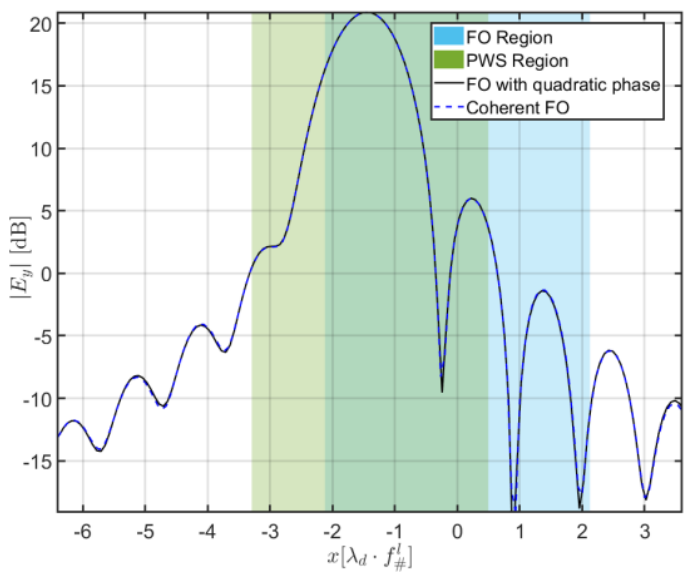

(a)

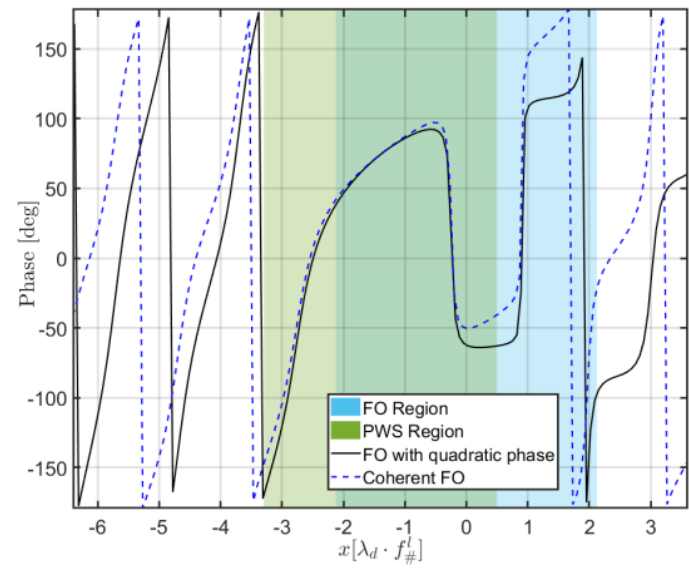

(b)

Figure K.2: The y-component of the electric fields on the focal plane of an elliptical silicon $\left(\varepsilon_{r}=11.9\right)$ lens with $D_{l}=5 \lambda_{0}\left(f_{0}=300 \mathrm{GHz}\right)$ and $f_{\#}^{l}=0.6$, illuminated by a unitary TE polarized plane wave with the skew angle of $\theta_{s}=16^{\circ}, \phi_{s}=0^{\circ}$. Here a quarter-wavelength matching layer at $300 \mathrm{GHz}$ made of Parylene $\left(\varepsilon_{m}=2.62\right)$ is applied. The linearization point is chosen the same the flash point, i.e. $\vec{\rho}_{o} \approx-1.4 \lambda_{d} f_{\#}^{l} \hat{x}$. And the focal plane field calculated by using the coherent FO is compared with the one obtained using the GO-FO: (a) Amplitude. (b) Phase. One of the main planes $(y=0)$ is shown. Blue region is the FO applicability region and green region is the PWS applicability region. 


\section{K.3 Hemispherical lens}

A hemispherical silicon $\left(\varepsilon_{r}=11.9\right)$ lens with $D_{h l}=5 \lambda_{0}\left(f_{0}=300 \mathrm{GHz}\right), f_{\#}^{h l}=0.6$, $R_{s p h}=2.6 \lambda_{0}$, and $L=0.362 R_{s p h}$ is introduced here. A quarter-wavelength matching layer at $300 \mathrm{GHz}$ made of Parylene $\left(\varepsilon_{m}=2.62\right)$ is applied. The lens is illuminated by a unitary TE polarized plane wave with the skew angle of $\theta_{s}=20^{\circ}, \phi_{s}=0^{\circ}$, i.e. $\vec{\rho}_{f p} \approx-1.75 \lambda_{d} f_{\#}^{h l} \hat{x}$. The linearization point is chosen the as the flash point. Fig. K.3 shows the y-component of the electric fields on the focal plane of the lens. The focal plane field evaluated by using the coherent FO is compared with the one obtained using the GO-FO. As it can be seen in the figure, the coherent FO is validated inside the PWS applicability region.

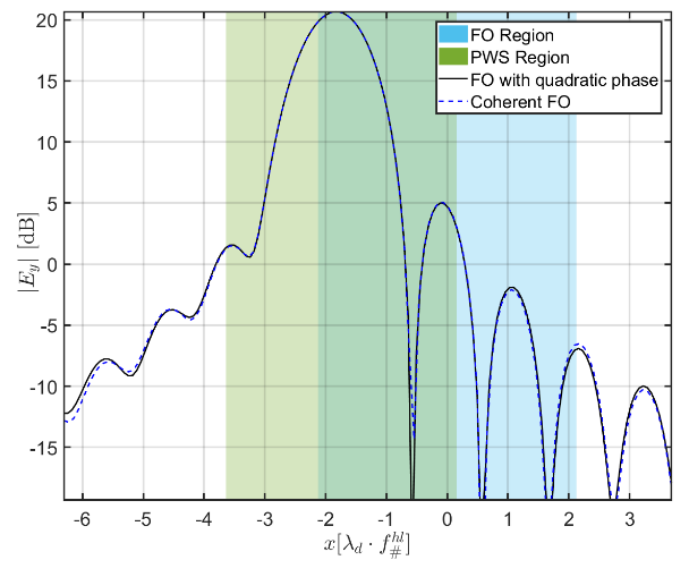

(a)

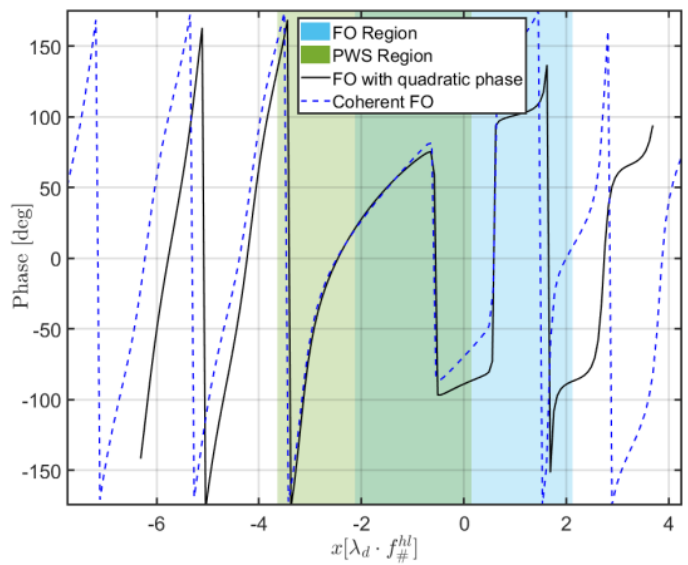

(b)

Figure K.3: The y-component of the electric fields on the focal plane of a hemispherical silicon $\left(\varepsilon_{r}=11.9\right)$ lens with $D_{h l}=5 \lambda_{0}\left(f_{0}=300 \mathrm{GHz}\right), f_{\#}^{h l}=0.6, R_{s p h}=2.6 \lambda_{0}$, and $L=0.362 R_{s p h}$, illuminated by a unitary TE polarized plane wave with the skew angle of $\theta_{s}=20^{\circ}, \phi_{s}=0^{\circ}$. Here a quarter-wavelength matching layer at 300 $\mathrm{GHz}$ made of Parylene $\left(\varepsilon_{m}=2.62\right)$ is applied. The linearization point is chosen the same the flash point, i.e. $\vec{\rho}_{o} \approx$ $-1.75 \lambda_{d} f_{\#}^{h l} \hat{x}$. And the focal plane field calculated by using the coherent FO is compared with the one obtained using the GO-FO: (a) Amplitude. (b) Phase. One of the main planes $(y=0)$ is shown. Blue region is the FO applicability region and green region is the PWS applicability region. 


\section{K.4 Hyperbolic lens}

A hyperbolic plastic $\left(\varepsilon_{r}=2\right)$ lens with $D_{h}=100 \lambda_{0}\left(f_{0}=300 \mathrm{GHz}\right)$ and $f_{\#}^{h}=1$ is introduced. The incident field is a unitary TE polarized plane wave with the skew angle of $\theta_{S}=$ $3.7\left(\lambda_{d} / D_{h}\right)=1.5^{\circ}, \phi_{s}=0^{\circ}$, i.e. $\vec{\rho}_{f p} \approx-3.7 \lambda_{0} f_{\#}^{h} \hat{x}$. The linearization point is chosen the as the flash point. Fig. K.4 shows the y-component of the electric fields on the focal plane of the hyperbolic lens. The focal plane field calculated by using the coherent FO is compared with the one obtained using the analytical FO. It can be seen that the coherent FO is validated inside the PWS applicability region.

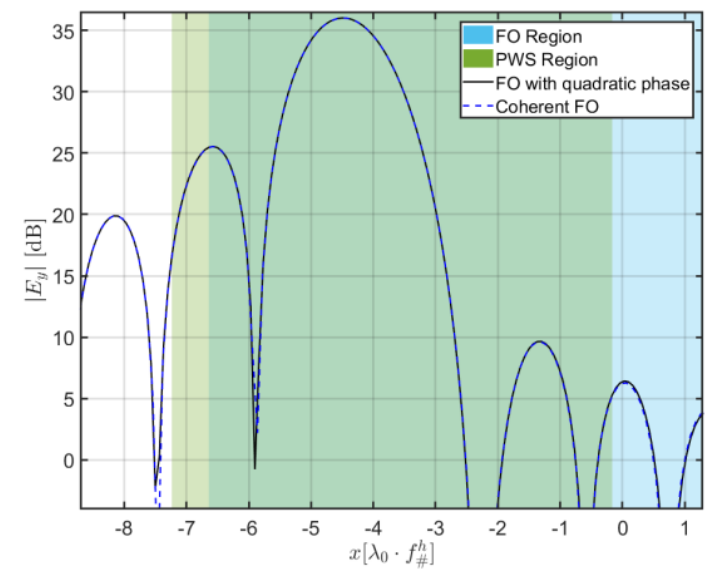

(a)

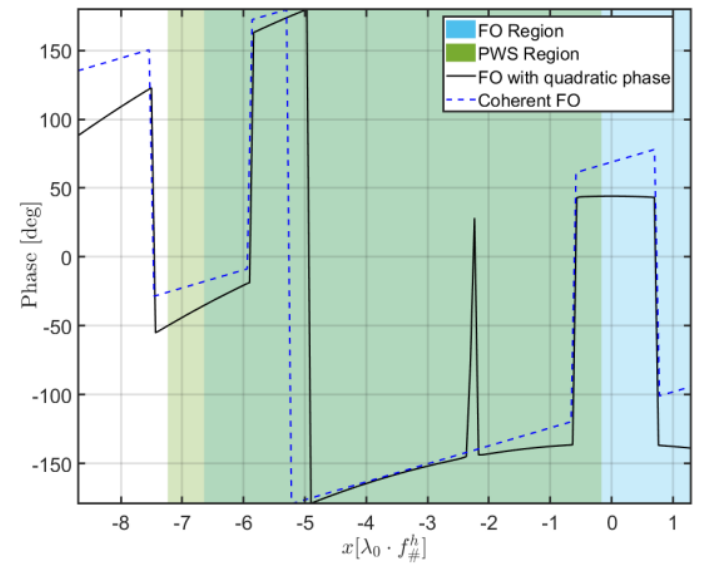

(b)

Figure K.4: The y-component of the electric fields on the focal plane of a hyperbolic plastic $\left(\varepsilon_{r}=2\right)$ lens with $D_{h}=100 \lambda_{0}\left(f_{0}=300 \mathrm{GHz}\right)$ and $f_{\#}^{h}=1$, illuminated by a unitary TE polarized plane wave with the skew angle of $\theta_{s}=3.7\left(\lambda_{d} / D_{h}\right)=1.5^{\circ}, \phi_{s}=0^{\circ}$. The linearization point is chosen the same the flash point, i.e. $\vec{\rho}_{o} \approx-3.7 \lambda_{0} f_{\#}^{h} \hat{x}$. And the focal plane field calculated by using the coherent FO is compared with the one obtained using the analytical FO: (a) Amplitude. (b) Phase. One of the main planes $(y=0)$ is shown. Blue region is the FO applicability region and green region is the PWS applicability region. 


\section{K.5 Elliptical Mirror}

Here we consider a mirror with the diameter of $D_{m}=100 \lambda_{0}\left(f_{0}=300 \mathrm{GHz}\right)$, the semimajor axis of $a=80 \lambda_{0}$, and the focal distance of $c=20 \lambda_{0}$, i.e. the eccentricity is $e=0.25$. A unitary Huygens source is placed at the lower focal plane $\left(f_{\#}^{m}=0.6\right)$, with the electric current oriented along $\hat{y}$ and a displacement in x-direction, $x_{s}=6.6 \lambda_{0} f_{\#}^{m}$. This displacement corresponds to a flash point $\vec{\rho}_{f p} \approx-4.5 \lambda_{0} f_{\#}^{m} \hat{x}$, and the linearization point is the same as this flash point. Fig. K.5 shows the y-component of the electric fields on the focal plane of the mirror, evaluated by using the coherent FO and compared with the analytical FO. It can be seen that the coherent FO is validated within the PWS applicability region.

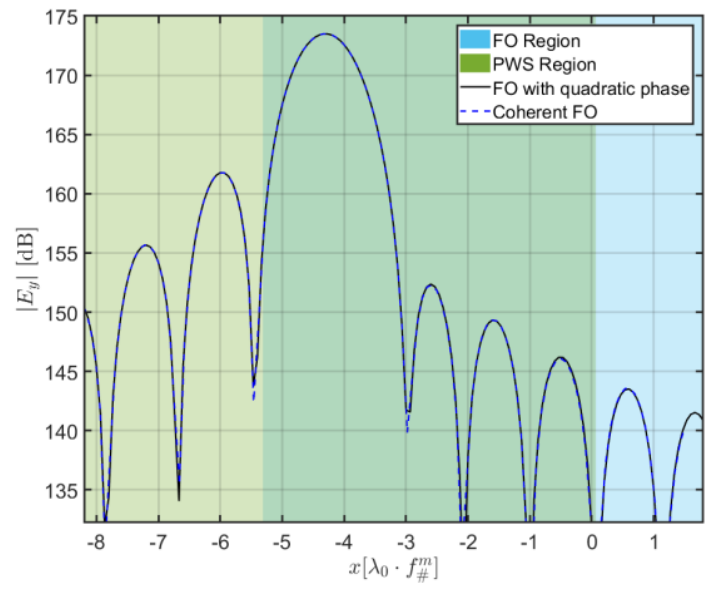

(a)

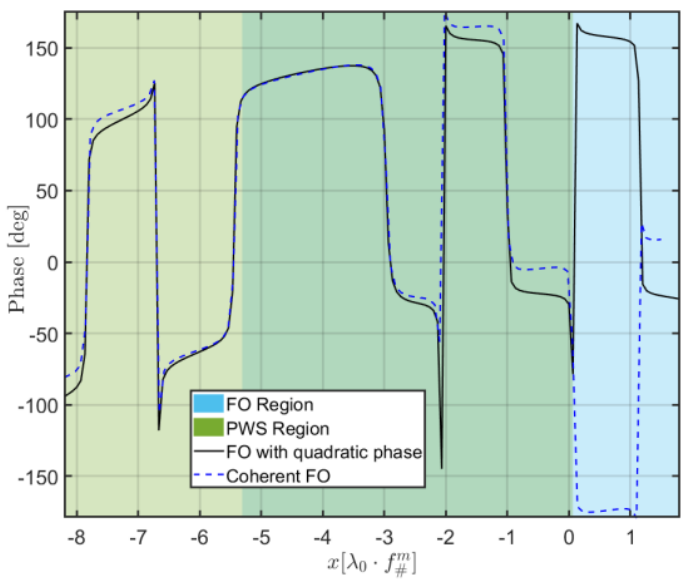

(b)

Figure K.5: The y-component of the electric fields on the focal plane of an elliptical mirror with $D_{m}=$ $100 \lambda_{0}\left(f_{0}=300 \mathrm{GHz}\right), f_{\#}^{m}=0.6$ and $e=0.25$. The mirror is illuminated by a unitary Huygens source placed at the lower focal plane, with the electric current oriented along $\hat{y}$ and a displacement in $\mathrm{x}$-direction, $x_{s}=$ $6.6 \lambda_{0} f_{\#}^{m}$. The linearization point is chosen the same the flash point, i.e. $\vec{\rho}_{o} \approx-4.5 \lambda_{0} f_{\#}^{m} \hat{x}$. And the focal plane field calculated by using the coherent FO is compared with the one obtained using the analytical FO: (a) Amplitude. (b) Phase. One of the main planes $(y=0)$ is shown. Blue region is the FO applicability region and green region is the PWS applicability region. 